\title{
Underwater acoustic communication over Doppler spread channels
}

\author{
by \\ Trym H. Eggen \\ Submitted in partial fulfillment \\ of the requirements for the degree of \\ Doctor of Philosophy \\ at the
Massachusetts Institute of Technology
and the \\ Woods Hole Oceanographic Institution \\ June 1997
}

(c) Trym H. Eggen, MCMXCVII. All rights reserved.

The author hereby grants to MIT and to WHOI permission to reproduce to distribute copies of this thesis document in whole or in part.
MBL/WHOI LIBRARY

Woods Hole, MA

Marine Biological Labúratory ar'Wods Hole Oceanographic Institution

Author

MIT Department of EECS and the MIT/WHOf Joint Program in Oceanography and Oceannographic Engineering, May 13, 1997

Certified by

Arthur B. $\overline{\overline{\text { Baggeroer }}}$

Ford Professor of Electrical Engineering and Ocean Engineering, MIT, Thesis

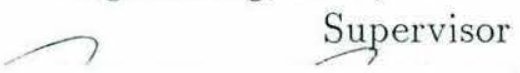

Certified by

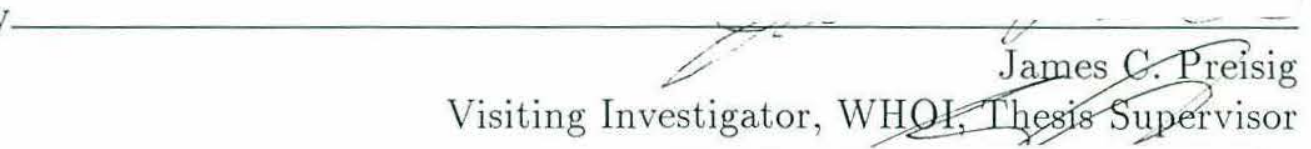

Accepted by

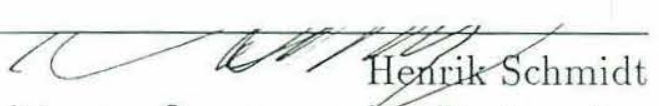

Acting Chairman, MIT/WHOI Joint Committee on Oceanographic Engineering

Accepted by

Arthur C. Smith

Chairman, MIT EECS Departmental Committee on Graduate Students 


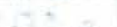

${ }^{3}+1,1$ 


\title{
Underwater acoustic communication over Doppler spread channels
} by Trym H. Eggen

\author{
Submitted in partial fulfillment of the \\ requirements for the degree of \\ Doctor of Philosophy \\ at the \\ Massachusetts Institute of Technology \\ and the \\ Woods Hole Oceanographic Institution \\ June 1997
}

\begin{abstract}
This work is concerned with coherent communication by means of acoustic signals over underwater communication channels. The estimated scattering functions of real data ranging from the Arctic environment to tropical waters show that underwater communication channels can not be captured by a single, simple channel model. This thesis considers mainly a subset of underwater communication channels where the Doppler spread is more severe than the delay spread.

An appropriate representation of the linear time-variant channel is introduced, and the wide sense stationary uncorrelated scattering (WSSUS) channel assumption enables characterization in terms of scattering functions. The concept of Doppler lines, which are frequency domain filters, is used in the derivation of a receiver for Doppler spread channels.

The channel is simulated by means of a ray representation for the acoustic field and a time-variant FIR filter. The impact of physical ocean processes on the Doppler spread is demonstrated, and from this modeling explanations for the Doppler spread observed in real data are obtained.

A decision feedback equalizer (DFE) adapted with recursive least squares (RLS) is analyzed, and its limit with respect to pure Doppler spread is found. By using the DFE with a phase locked loop (PLL) suboptimal system behavior is found, and this is verified on real data. In the case of a simple Doppler shift the cross-ambiguity function is used to estimate the shift, and the received signal is phase rotated to compensate this before it enters the receiver.

A modified RLS called the time updated RLS (TU-RLS) is presented, and it is
\end{abstract}


used in a new receiver. This receiver is initialized by means of the cross-ambiguity function and the performance is characterized by probability of decoding error vs delay spread, Doppler spread and SNR. The receiver uses Doppler lines to compensate both discrete and continuous Doppler spread. The receiver stability depends on the conditioning of the block diagonal correlation matrix propagated by the TU-RLS. The receiver is used to decode both real and simulated data, and some of these data are severely Doppler spread.

Thesis Supervisor: Arthur B. Baggeroer

Title: Ford Professor of Electrical Engineering and Ocean Engineering, MIT

Thesis Supervisor: James C. Preisig

Title: Visiting Investigator, WHOI 


\section{Acknowledgments}

This part of my thesis could easily have been several times longer. There are so many different persons that have contributed in various stages of this work, and I really would like to thank them all individually. In particular I would like to thank my supervisors and thesis committee members for their contributions.

My discussions with Art Baggeroer have been very helpful throughout my time at MIT. He has a very well developed sense for pointing out the issues of genuine importance in relatively complex discussions. He also know exactly when a student needs challenging words as well as when the need is there to let him have more time to reflect on the work. My interaction with Art has been partly influenced by illness restraining him in periods from being present. In spite of this I am left with the conviction that I could not have found anybody serving my needs at the various stages of my work better. Art is dedicated to always get the best possible result, and I have been lucky to benefit greatly from this.

Also my other supervisor Jim Preisig deserves my heartfelt thanks. The advice he has provided in my research is hard to value high enough. I know him as a professionally very thorough person, and he has repeatedly enriched my research by applying this as well as other features. I will especially remember him for the very gentle and insightful way in which he has managed to transit between being my only supervisor and being my co-supervisor.

Mitch Trott has served as a valuable discussion partner, and his approach to my questions has more than once helped my to gain deeper insight. His never failing willingness to discuss has been of great benefit to me. I am indebted to Jim Lynch for his great expertise on oceanography which he has put forward to me in a very gentle and wise way. His way of interacting has stimulated my interest and increased my insight in these matters.

I have experienced the process of gaining a doctoral degree as challenging in very diverse ways. In the same way as my committee has served to help me in dealing with the professional challenges, my family has served an equally important role of looking after me in various other ways. The patience, good spirit, faithfulness and encouragement of my wife Sissel has been crucial in seeing this work through. Also my two sons Tormod and Vemund have contributed significantly to help me keep the right perspective on things. I am also indebted to my mother, father and sister for the blessing they generate in my life.

I have been provided with very good working environments throughout the academic career both at MIT and WHOI. The acoustics group and the signal processing group, both at MIT, have been places where I could share thoughts, have discussions and cooperate with fellow students, and it has meant much to me. I want to extend my thanks to Kathleen Wage, Brian Sperry, Brian Tracey, John Buck and JT Goh for providing all sorts of help that can not easily be asked from anybody else than 
other fellow students. I am very grateful for their presence in my daily life.

During these four years I have been enriched by conversations with a number of faculty members both at WHOI and MIT. Henrik Schmidt and Al Oppenheim provided some very important support and advice at critical stages and I am not sure where I would have been without this. George Frisk has been a patient discussion partner whose advice has helped a lot. Ann Henry has solved many issues of administrative importance for me. Sabina Rataj and Isela Cordova have helped me to meet administrative challenges, and their true concern and endless patience have made a big difference.

Also my two years and three summers at WHOI are filled with memories of good friends and invaluable help. The friendship of Peter Traykovski, Dan Kilfoyle, Janet Fredericks and Bob Headrick has been a source of energy and joy. My many discussions with these friends have contributed to my work. Also the help with computers from Cindy Sellers, Art Newhall and Matt Grund has kept my work flowing smoothly and steadily. Mark Johnson and Lee Freitag have played key roles in all the practical work conducted at WHOI. Their generosity, practical insight and hands on experience with the equipment is valued very high. I am also indebted to Josko Catipovic for generously giving me access to many of the previously recorded real data sets. I could not have verified any of my thoughts on real data without their ample help. Also Eddie Scheer and Keith Von der Heidt have been especially supportive and helpful. These guys never said "no" when I came along asking for something, and I benefited from their hardware support, computer resources as well as friendship and humor.

The administrative work at WHOI has been well taken care of by the education office, and I especially remember Jake Peirson for his encouragement and never failing support in my work.

The work in this thesis was funded in part by the Norwegian Research Counsil under a general $\mathrm{PhD}$ contract, the education office of Woods Hole Oceanographic Institution and the Office of Naval Research under contracts N00014-95-0153 and N00014-95-1-0322. 
... I seem to have been

only like a boy playing on the seashore, and diverting myself now and then finding

a prettier shell than ordinary whilst the great ocean of truth lay all undiscovered before me

Sir Isaac Newton 


\section{Contents}

1 Introduction $\quad 27$

1.1 Background ......................... 27

1.1.1 Short range, line of sight based systems . . . . . . . . . . . . 30

1.1.2 Medium range, reverberation limited systems . . . . . . . 31

1.1 .3 Long range . . . . . . . . . . . . . . . . . . 31

1.1.4 Simulation studies . . . . . . . . . . . . . . . 32

1.2 The problem of underwater communication . . . . . . . . 35

1.2.1 A subset of communication channels . . . . . . . . . . . . 38

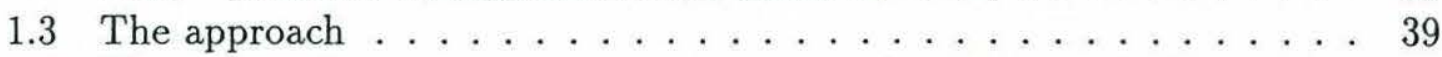

1.4 Thesis overview . . . . . . . . . . . . . . . . . . 43

2 Characterization of time-variant channels 47

2.1 Preview and motivation . . . . . . . . . . . . . . . 47

2.2 Wide sense stationary uncorrelated scattering channel . . . . . . . . 48

2.2.1 Representation of the transmit signal . . . . . . . . . . . . . 52

2.3 Ambiguity function . . . . . . . . . . . . . . . . . . . 55

2.3.1 Time-variant and multiple Doppler shifts . . . . . . . . . . . 61

2.3.2 Narrowband assumption . . . . . . . . . . . . . 64

2.4 Doppler lines and delay lines . . . . . . . . . . . . . . . . . . 66

3 Simulations and measurements of communication channels $\quad 79$

3.1 Simulation tool . . . . . . . . . . . . . . . 79

3.1.1 Time-variant FIR model . . . . . . . . . . . . . . . . . 79

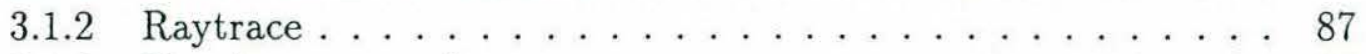

3.1.3 Simulator examples . . . . . . . . . . . . . . . . 91

3.2 Measured scattering functions . . . . . . . . . . . . . . . . . . . . . . 99

$3.2 .1 \quad$ Arctic data . . . . . . . . . . . . . . . . . . . 99

3.2 .2 Autec data ... . . . . . . . . . . . . . . 104

3.2.3 Florida data. . . . . . . . . . . . . . . . . 108

3.2 .4 Gould Island data . . . . . . . . . . . . . . . . . . . . 112

$\begin{array}{llr}4 & \text { Receiver } & 117\end{array}$ 
4.1 Maximum likelihood receiver . . . . . . . . . . . . . . . . . 117

4.1 .1 Preview and motivation . . . . . . . . . . . . 117

4.1 .2 Doubly spread channel . . . . . . . . . . . . . . . . 118

4.1 .3 Delay spread channel . . . . . . . . . . . . . . . . . . 119

4.1.4 Doppler spread channel . . . . . . . . . . . . . . . . . . 121

4.1.5 Training vs tracking . . . . . . . . . . . . . 122

4.2 Doppler analysis of a common receiver . . . . . . . . . . 129

4.2 .1 Preview . . . . . . . . . . . . . . . . 129

4.2 .2 Signal models . . . . . . . . . . . . . . . . . 130

4.2 .3 RLS algorithm . . . . . . . . . . . . . . . 132

4.2 .4 Receiver structure . . . . . . . . . . . . . . . . 137

4.2 .5 Phase locked loop . . . . . . . . . . . . . . . . . . 144

4.2.6 Interaction between phase locked loop and filter adaptation . . 149

4.2.7 Simulation and real data examples . . . . . . . . . 157

4.3 A receiver for doubly spread channels . . . . . . . . . . . . . . . 164

4.3.1 Channel model . . . . . . . . . . . . . . . . . . . . 164

4.3.2 The modified RLS algorithm . . . . . . . . . . . . 167

4.3.3 Receiver for one Doppler shift at each delay . . . . . . . . 169

4.3.4 Generalized receiver . . . . . . . . . . . . . . . 171

4.3 .5 Signal combiner . . . . . . . . . . . . . . . . . . 188

4.3.6 Tap initialization . . . . . . . . . . . . . . . . . 192

4.3 .7 Tap tracking . . . . . . . . . . . . . . . . . 211

4.3 .8 Receiver robustness . . . . . . . . . . . . . . . . . 213

4.4 Verification of the receiver . . . . . . . . . . . . . . . 239

4.4 .1 One Doppler shift for each delay . . . . . . . . . . . . 239

4.4.2 Multiple Doppler shifts for each delay . . . . . . . . . . . 247

4.4 .3 Real data . . . . . . . . . . . . . . . . . . 262

5 Conclusion 271

5.1 Summary of thesis . . . . . . . . . . . . . . 271

5.1 .1 Background . . . . . . . . . . . . . 271

5.1 .2 Theory . . . . . . . . . . . . . . . . . . . 272

5.1 .3 Channel modeling . . . . . . . . . . . . . . . . . 272

5.1.4 Receiver derivation and analysis . . . . . . . . . 273

5.2 Future directions . . . . . . . . . . . . . . 275

5.2.1 DFE adapted with TU-RLS . . . . . . . . . 275

5.2 .2 Error coding . . . . . . . . . . . . . . 276

5.2 .3 Simulation . . . . . . . . . . . . . . 276

5.2 .4 Modulation . . . . . . . . . . . . . . . . . 277

5.2.5 Combined spatial and temporal processing . . . . . . . 277

5.2 .6 Channel representation . . . . . . . . . . . . 278 
$\begin{array}{ll}\text { A Stability of the stationary region } & 279\end{array}$

A.1 Angle PD . . . . . . . . . . . . . . . . 280

A.2 Imaginary part PD . . . . . . . . . . . . 281

B Channel estimation error covariance 283

C Approximations in computing the hypothesis probability 291 


\section{List of Figures}

1-1 An overview of the proposed system for use in the underwater communication channel. . . . . . . . . . . . . . . 42

2-1 Recovering the information sequence from the carrier modulated complex envelope. . . . . . . . . . . . . . . 54

2-2 Channel identification problem, no inversion needed because both channel input and output are known. . . . . . . . . 58

2-3 The complex envelope (upper panel) and $3 \mathrm{~dB}$ contours of the crossambiguity function (lower panel) for a signal with time-variant Doppler. 62

2-4 The complex envelope (upper panel) and $3 \mathrm{~dB}$ contours of the crossambiguity function (lower panel) for a signal with two Dopplers. . . . 63

2-5 The FFS Doppler line. . . . . . . . . . . . . . . 71

2-6 The convolution of the two signals yields the output of the FFS Doppler line. . . . . . . . . . . . . . . . . . 72

2-7 The IFS Doppler line. . . . . . . . . . . . . . . . 74

3-1 The simulator is based on ocean related parameters feeding a raytrace model. . . . . . . . . . . . . . . . . . . . . 80

3-2 Time varying, clustered channel with four taps in the FIR filter. . . . 81

3-3 The delay structure of the received signal can be derived from the raytrace picture. . . . . . . . . . . . . . . . 82

3-4 Surface Doppler spread, a snapshot of the time-variant rough surface which is modeled as a random process. We use the time-averaged reflection coefficient, therefore only the specular direction is shown . . 83

3-5 Average reflection coefficient vs grazing angle (angle with respect to the horizontal plane). The curves are parameterized by the rms roughness $s 0$ going from $0.05-0.5 \mathrm{~m} . \ldots \ldots \ldots \ldots \ldots$

3-6 A simplified representation of the impact of a rough time varying surface on a ray. . . . . . . . . . . . . . . .

3-7 Isovelocity example, there is no motion of receiver or transmitter and the vertical beamwidth of the transmitter prevents boundary interaction. The extent of the ambiguity function is given by signal bandwidth and duration. . . . . . . . . . . . . . . 94 
3-8 Moving transmitter, no receiver motion. . . . . . . . 95

3-9 Sound speed profile taken off New Jersey during summer conditions. . 96

3-10 Sound speed profile taken outside Newport (RI) during an experiment in the winter. . . . . . . . . . . . 97

3-11 An example of a Doppler spread built up of separate Doppler shifts that are different on different ray paths. . . . . . . . . 98

3-12 The geometry of the Arctic experiment. Both transmitter and receiver were suspended from the ice. . . . . . . . . . . 100

3-13 The ambiguity function of the transmitted signal. The extent in time is given by the signal bandwidth and the extent in frequency is given by the signal duration. . . . . . . . . . . . . . . . 101

3-14 Impulse response and channel scattering function for $300 \mathrm{~m}$ range. . . 102

3-15 Impulse response and channel scattering function for $3 \mathrm{~km}$ range. . . 103

3-16 The geometry of the Autec experiment. The transmitter is suspended from a boat and the receiver is bottom mounted. . . . . . . . 104

3-17 Impulse response and scattering function estimates. The channel has a direct path and a surface reflected path that has been Doppler and delay spread due to the surface motion. Three contour levels with distance between contours $3 \mathrm{~dB}$ are shown. . . . . . . . . 106

3-18 Series of cross-ambiguity functions containing the direct path and a surface reflected path that has been Doppler and delay spread due to the surface motion. Three contour levels with distance between contours $3 \mathrm{~dB}$ are shown. . . . . . . . . . . . . 107

3-19 The geometry of the Florida experiment. Both transmitter and receiver are suspended from moving boats. . . . . . . . . . . 108

3-20 The impulse response and channel scattering function for short range $(300 \mathrm{~m})$ data, showing one direct and one surface reflected path. . . 110

3-21 The impulse response and channel scattering function for long range $(3 \mathrm{~km})$ data, showing one direct and one possibly bottom reflected path.111

3-22 The geometry of the Gould Island experiment. The transmitter is suspended from a boat and the receiver is mounted in a dock. . . . 112

3-23 The complex envelope of real and simulated data. Two paths with different Doppler shifts create a beating frequency. . . . . . . . . . 114

3-24 Impulse response and channel scattering function of Doppler spread data from transmitter movement. Three contour level with spacing 3 $\mathrm{dB}$ are shown in the lower panel. . . . . . . . . . . 115

4-1 Data packet format containing synchronization, training sequence used for the cross-ambiguity function and the TU-RLS convergence and data symbols to be decoded. . . . . . . . . . . . . . . . . 123

4-2 The time-variant channel modulates the received signal, but the channel variation is much slower than the symbol rate. . . . . . . . . 124 
4-3 The scaling factor of the steady state solution vs $\lambda$ with the number of taps $L$ as parameter. . . . . . . . . . . . . . . 136

4-4 A single channel receiver, all parameters adapted with RLS. . . . . 138

4-5 The matched receiver that must handle the Doppler shift in the input signal. With no ISI the feedback part of the DFE is absent. . . . . 139

4-6 The equivalent RLS phase locked loop. $\Sigma$ is a summer (discrete integrator). . . . . . . . . . . . . . . . . . 140

4-7 Magnitude (top) and phase of the filter determining the filter tap $a(n)$ when the RLS algorithm is tracking a constant Doppler. . . . . . . 141

4-8 The filter tap is generated from a low pass filter driven by a complex sinusoid that is the Doppler shift. . . . . . . . . . . . . . . . . . 142

4-9 The maximum Doppler shift that can be corrected by the RLS-PLL is a function of the forgetting factor $\lambda \ldots \ldots \ldots \ldots \ldots$

4-10 Building blocks of a PLL. . . . . . . . . . . . . . 145

4-11 The structure of the digital PLL, working on complex signals. $\Sigma$ is a summer (discrete integrator). . . . . . . . . . . . . 147

4-12 The phase and modulus of the signal going into the PLL is found by considering these structures. . . . . . . . . . . . . . . . 148

4-13 The composite receiver, consisting of a decision feedback equalizer and a phase locked loop. . . . . . . . . . . . . . . . . 150

4-14 10 trials of the state trajectories when perturbing the filter tap with a small random value $\delta a$ away from the stationary region. The top picture is PLL phase estimate, and the bottom picture is the complex filter tap values. . . . . . . . . . . . . . . . . 158

4-15 10 trials of the state trajectories when a linear phase shift is applied to the input and the filter tap is perturbed away from the stationary region. The PLL phase estimate at the top, and the complex filter tap values at the bottom. The time arrows of all the trajectories of $a(n)$ are pointing toward the middle where the $\operatorname{Re}(a)=1 . \ldots \ldots 159$

4-16 The filter tap rotates at a constant rate, taking care of some of the Doppler at the expense of its amplitude. The rest of the Doppler is compensated by the PLL. It is arbitrary how the Doppler is shared between the PLL and the filter tap. There are three cases of Doppler handled by the RLS, $\omega_{0}=\left[\begin{array}{lll}.001 & .01 & .05\end{array}\right] \ldots \ldots \ldots \ldots \ldots$. . . . 160

4-17 The multichannel version of the DFE, running on four data channels. The RLS is jointly adapting all filter coefficients. . . . . . . . . . 161

4-18 The PLL output phase estimate $\hat{\phi}(n)$ when applied to channels 1-4 (left) and channels 1-2 (right) of real data. The four channels are believed to have the same Doppler because they were recorded from elements closely spaced and mounted on a rigid platform, but the PLL estimates are different. The two channels 1 and 2 are estimated differently when channel 3 and 4 are not used. . . . . . . . . 162 
4-19 The taps of the 4 channel equalizer running on real data. All taps are rotating. The magnitudes of the taps are significantly different, even though the noise has similar variance on all the channels. The highest magnitude tap is on the channel where the PLL phase estimate is largest. . . . . . . . . . . . . . . . . . 163

4-20 Receiver built up of separate channel tracker and equalizer. . . . . . 169

4-21 The channel tracking of a doubly spread channel implemented with block processing.

4-22 The channel tracking of a doubly spread channel implemented with recursive processing. . . . . . . . . . . . . 176

4-23 Decoding using FFS Doppler lines. . . . . . . . . . . . . . . . 182

4-24 The FFS based receiver for a doubly spread channel. . . . . . . . 185

4-25 The FFS based receiver for a purely Doppler spread channel. . . . . . 186

4-26 Contour plot of the cross-ambiguity function computed from the training sequence and the received data. There are 5 symbol intervals between the arrival clusters at 3 and 5 msec. . . . . . . . . . . . 193

4-27 The probability that a certain location in the cross-ambiguity function corresponds to a signal return vs the value of the cross-ambiguity function. There are $L=9$ unit strength scatterers at other locations and the scatterer at $(0,0)$, which we are trying to detect has strength 0.5 . The normalization of the first axis is relative the ambiguity function peak value. . . . . . . . . . . . . . . . 207

4-28 The number of taps used by the receiver vs $\lambda$ for a Doppler spread of

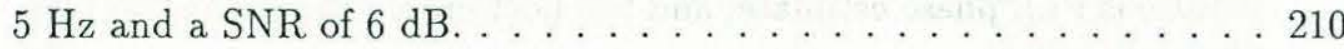

4-29 Contours of maximum $\lambda$ for a given symbol rate and Doppler frequency mismatch that keep the amplitude of $\hat{h}(n)$ above $90 \%$ of $h(n) .216$

4-30 Simulation of the channel estimation error covariance for a purely Doppler spread channel. The lower panel shows the comparison between the closed form and simulated channel estimation error covariance.229

4-31 The theoretical total channel estimation error variance vs the number of taps $L$ and the forgetting factor $\lambda$. The increase of the variance for low $\lambda$ and high $L$ shows the receiver limitation due to ill conditioning of $\tilde{P}$. The Doppler spread is $5 \mathrm{~Hz}$ and the SNR is $6 \mathrm{~dB} \ldots \ldots 232$

4-32 The condition number of $\tilde{P}$ vs the number of taps $L$ for different forgetting factors $\lambda$ and a Doppler spread of $5 \mathrm{~Hz}$ at a symbol rate of $2500 \mathrm{symb} / \mathrm{sec}$

4-33 The total channel estimation error variance vs the number of taps $L$ and the forgetting factor $\lambda$ obtained by running the receiver on Doppler spread data. The increase of the variance for low $\lambda$ and high $L$ is consistent with the theoretical prediction, and it shows the receiver limitation due to ill conditioning of $\tilde{P} \ldots \ldots . \ldots 234$ 
4-34 The probability of decoding error vs Doppler spread for a purely Doppler spread channel with the SNR as parameter. The symbol rate is assumed to be $600 \mathrm{symb} / \mathrm{sec}$. The dotted lines are the probability of error when the decision errors are not fed back to the channel tracker.

4-35 The probability of decoding error vs Doppler spread for a doubly spread channel with the SNR as parameter. The symbol rate is assumed to be $600 \mathrm{symb} / \mathrm{sec}$ and the delay spread is $5 \mathrm{msec}$. The dotted lines are the probability of error when the decision errors are not fed back to the channel tracker. . . . . . . . . . . . . .

4-36 Decoding of Doppler spread data, the ambiguity function shows three returns at different Doppler shifts (upper left panel), the TU-RLS taps are shown to rotate at different amplitudes (upper right panel), and the eye pattern showing the decoding result is open (lower left panel). . . . . . . . . . . . . . . . . . . . . . 243

4-37 Decoding of doubly spread data where the FIR signal combiner has more difficulty because there are returns of almost equal amplitude at different delays. . . . . . . . . . . . . . . . . . 244

4-38 Decoding of a typical sparse underwater communication channel with many symbol intervals between the returns and the returns at different Doppler shifts. The second return is from a surface swell with much longer period than the packet length, so that a Doppler shift rather than a spread is the result. . . . . . . . . . . . . . 245

4-39 Performance of the DFE receiver with a PLL on a Doppler spread signal. The predicted symbols in the right column and the MSE decision error in the right column for a two path signal with respectively 0,1 and $2 \mathrm{~Hz}$ difference in Doppler between the paths in the upper, middle and lower panels. . . . . . . . . . . . . . . . . 246

4-40 Decoding using the IFS Doppler line and recursive channel tracker. The tap locations are found by thresholding the cross-ambiguity function in the upper left panel. The initial convergence of the TU-RLS from training data is shown in the first 256 symbols in the upper right panel. . . . . . . . . . . . . . . . . . . 251

4-41 Decoding using the IFS Doppler lines and the block channel estimator. There are two rays with different frequencies. . . . . . . . . . . 252

4-42 Decoding using the IFS Doppler lines and the block channel estimator. There are four rays with different frequencies. . . . . . . . . 253

4-43 Decoding of purely Doppler spread data with an FFS Doppler line. There are 2 frequencies in the received data. The channel crossambiguity function in upper left panel, the FFS coefficients in upper right panel and the decoded symbol estimates in the lower left panel. 
4-44 Decoding of Doppler spread data with a constrained FFS Doppler line where the constraint is to have unity white noise Doppler line gain. The channel has two frequencies with almost equal magnitude. . . . . 255

4-45 Decoding of Doppler spread data with an FFS Doppler line. There are 4 Doppler shifts in the received signal. The channel cross-ambiguity function in upper left panel, the FFS coefficients in upper right panel and the decoded symbol estimates in the lower left panel. . . . . . . 256

4-46 Decoding of Doppler spread data with a constrained FFS Doppler line where the constraint is to have unity white noise Doppler line gain. There are 4 Doppler shifts in the received signal. The channel crossambiguity function in upper left panel, the FFS coefficients in upper right panel and the decoded symbol estimates in the lower left panel.

4-47 Decoding using the IFS Doppler line and recursive channel tracker. The Doppler spread is generated from band limited white noise. . . . 259

4-48 Decoding using the FFS Doppler lines and the block channel estimator. The channel response contains a continuous Doppler spread with nearly flat spectrum over its bandwidth. . . . . . . . . . . . . . 260

4-49 Probability of decoding error vs Doppler spread when there is no delay spread and the SNR is $12 \mathrm{~dB}$. . . . . . . . . . . . . 261

4-50 Map over the site of the Gould Island experiment. The island intersected by the lower border of the rectangle is Gould Island where the receiver is mounted. . . . . . . . . . . . . . . . . 262

4-51 Eye pattern from the decoding of some data transmissions in the ocean. The left column shows results obtained with the DFE, and the right column shows results when using the new receiver.

4-52 Decoding and channel tracking of purely Doppler spread data from the Gould Island experiment. The mean Doppler is from transmitter vessel drift.

4-53 The transmitting vessel is anchored, but it has a roll producing Doppler spread. . . . . . . . . . . . . . . . 266

4-54 The transmitting vessel was drifting, and the received signal level is very sensitive to transmitter depth. . . . . . . . . . . . 267

4-55 Snapshots of impulse responses from real data with one dominant signal return which is fading. . . . . . . . . . . . . . . 268

4-56 Time evolution of a Doppler spread signal return from the Gould Island experiment. . . . . . . . . . . . . . . . . . . . . 269

A-1 The filter tap and the PLL phase estimate is in equilibrium when the filter tap $a$ is on the unit circle. . . . . . . . . . . . . . . 279

C-1 Sketch of the probability densities of $\theta_{1}$ and $\theta_{2}$. . . . . . . . 296 


\section{List of Tables}

1.1 Classification of different communication channels. . . . . . . . . 29

4.1 The major steps and the associated equations in the derivation of the tap initialization rule. . . . . . . . . . . . . 195

4.2 The energy in $\hat{h}(n)$ for different cases of tap selection . . . . . 200 


\section{Notation glossary}

This is an overview of the most commonly used symbols in this thesis with a brief explanation of each symbol. In general boldface symbols means vector quantities. Regular parentheses () are used for both continuous and discrete quantities, and the argument and context carries the information to distinguish these cases. The symbol $j=\sqrt{-1}$.

$\mathbf{a}(n)$ : DFE coefficients. This is the filter coefficients of the decision feedback equalizer. The feedforward and feedback coefficients are concatenated.

$A(t)$ : Signal amplitude envelope. This is used in the complex representation of the baseband transmitted signal.

$A, A_{0}$ : System matrix. The state space description of the channel as well as some of the receiver algorithms use a channel model involving this matrix.

$\alpha: \operatorname{AR}(1)$ parameter. This determines the bandwidth of the Doppler spread modeled'as an $\mathrm{AR}(1)$ process.

$B$ : Doppler bandwidth. The frequency support of the time-variant modulation induced by the channel at a single delay.

$b$ : Reflection coefficient. A random variable modeling the varying reflection strength of a scatterer.

$\beta$ : Attenuation. The frequency and range dependent attenuation of the acoustic signal.

$c$ : The speed of sound. The propagation speed of the acoustic signal carrying the communication signal through the water.

$\mathbf{c}(n), \mathbf{c}(n, l)$ : Observation vector. Containing the signal that is used to update the recursive algorithms for receiver adaptation.

$\mathcal{E}$ : Signal energy. The energy used to transmit one information symbol.

$\delta(t):$ Dirac delta.

$\delta(n)$ : Kronecker delta.

$\Delta \nu$ : Tap frequency spacing. The distance between adjacent taps in the Doppler lines used in the receiver to compensate Doppler spread.

$e(n)$ : Prediction error. The difference between the received signal and its estimate generated in the receiver.

$F(k)$ : Doppler line coefficients. The $k^{\prime}$ th complex tap value of a Doppler line.

$f(n)$ : Inverse FFT of $F(k)$. The time-variant gain multiplying the signal entering a Doppler line.

$f_{s}$ : Sample frequency. The frequency used in the receiver to sample the received signal.

$f_{c}$ : Carrier frequency. The frequency with which the information symbols is transmitted.

$\mathbf{f}(\cdot)$ : Vector of functions.

$\phi(t), \phi(n)$ : Signal phase. The phase part of the complex envelope representation of 
the signal which carries the information in the coherent systems considered here. $\gamma(n)$ : Loop transfer function. The shaping filter in the phase locked loop used for Doppler tracking.

$\gamma$ : Modulation constant. A multiplier used in the receiver robustness derivation depending on the modulation format used.

$h(t, \tau), \mathbf{h}(t), \mathbf{h}(n), h(n, m)$ : Input delay-spread function. Used to characterize the time variant channel in a similar manner as the time-variant impulse response.

$\hat{\mathrm{h}}(\tau), \hat{\mathrm{h}}(n), \hat{h}(n, m)$ : Input delay-spread function estimate. Generated in the receiver in order to track the channel.

$\hat{h}_{0}(n, m), \hat{h}_{0}(n)$ : Decoder filter coefficients. The Wiener filter implemented in the decoder at each time instant based on the input delay-spread function estimates.

$h_{e}(n)$ : Channel estimation error. The difference between the true channel and the receiver estimate.

$H\left(e^{j \omega}\right)$ : Frequency response. Digital filter gain and phase.

${ }^{H}$ : Complex conjugate transpose. The Hermitian of vectors and matrices. $I$ : Identity matrix.

$J(\cdot)$ : Cost function. A quadratic error measure, usually a difference between a quantity and its estimate.

$\mathrm{k}(n)$ : Gain in RLS and TU-RLS. The factor that multiplies the prediction error when an estimate is updated.

$\chi$ : Steady state scaling factor. The recursive algorithms achieve a steady state after going through a startup transient.

$\psi$ : Grazing angle. The angle between the horizontal plane and a ray reflected from the ocean-surface or bottom.

$\lambda$ : Exponential weighting factor. The factor used in the recursive algorithms in order to forget the past measurements.

$L$ : Number of coefficients, number of rays. The number of taps used in the receivers, and also the number of rays contributing to the received signal.

n : Delay. Discrete formulation.

$p(\cdot)$ : Probability density.

$P, P(n), \tilde{P}:$ Matrix propagated in RLS and TU-RLS. This matrix determines the gain used in the update of channel estimates.

$P_{e}$ : Probability of decoding error. The probability that the receiver makes an error. $\Pi(n)$ : Channel estimate error covariance. The matrix yielding the error covariances at each time step in the recursive algorithm.

$\hat{\Pi}(n)$ : Channel estimate error covariance approximation. A matrix obeying a simpler difference equation than $\Pi(n)$, yet a good approximation.

$q$ : Wave number. The spatial wavelength of the acoustic signal.

$Q(\cdot)$ : QPSK quantizer. The nonlinear device mapping the receiver symbol estimate into the closest of symbols in the alphabet.

$Q_{0}$ : Autocorrelation matrix. The autocorrelation of the estimated symbols in $\mathbf{c}(n)$. $Q_{e}$ : Autocorrelation matrix. The autocorrelation of the decision errors. 
$R_{s s}(\tau), R_{h h}(\tau), R, R_{c}, R_{x x}(\tau), R_{y y}(n), R_{w w}, R_{U}(\xi, \eta, \nu, \mu), R_{h}(\tau, \eta, \xi)$ : Autocorrelation. The subscript when present indicates the name of the random variable in question. $\mathrm{r}, R_{x y}(\tau)$ : Cross correlation.

$S(\xi, \nu)$ : Scattering function. The distribution of energy in delay and Doppler as dispersed by the channel.

$s(t)$ : Ocean-surface Doppler spreading function. A random process modeling the Doppler spread due to the time-variant ocean-surface.

$\sigma_{w}^{2}, \sigma_{e}^{2}, \sigma_{y}^{2}, \sigma_{\hat{y}}^{2}$ : Variance. The subscript indicates the random variable in question.

$T$ : Time interval.

$\tau$ : Delay. Continuous formulation.

$T(f, t)$ : Time variant transfer function. The frequency response of a linear timevariant system.

$\theta_{0}(\tau, \nu), \theta_{0}(n, k)$ : Ambiguity function. Used for transmit signal characterization.

$\theta(\tau, \nu), \theta_{0}(n, k)$ : Cross-ambiguity function. Used for scattering function estimation.

$x(t)$ : Channel input, transmitted data. The signal that is output from the trans-

mitter into the underwater communication channel.

$X(f)$ : Fourier transform of $x(t)$.

$y(t), y(n), \mathbf{y}:$ Channel output, received data. The signal that is propagated over the channel and is recorded in the receiver.

$Y(f)$ : Fourier transform of $y(t)$.

$z(n), \mathrm{z}$ : Transmitted symbol. One of four complex numbers in the case of QPSK that we use.

$\hat{z}(n)$ : Soft estimate of symbol. The receiver decoder filter output.

$\tilde{z}(n)$ : Hard (quantized) estimate of symbol. The output of the receiver quantizer.

$z_{e}(n)$ : Quantization error. The difference between the hard estimate of the symbol and the transmitted symbol.

$\zeta$ : Condition number. The ratio of the largest to the smallest eigenvalue of a matrix. $U(\tau, \nu), U(l, k), U_{l, k}(n), \mathbf{U}(n)$ : delay-Doppler-spread function. A representation of the time-variant channel interpreted as the scattering strength at a specific delay and Doppler.

$u_{i}^{2}$ : Scattering strength. The variance of the delay-Doppler-spread function.

$\nu, \nu$ : Doppler frequency.

$v_{s}$ : Speed. The speed of scatterers in the channel.

$v(n), \tilde{v}(n), \mathbf{v}, w_{\theta}(n, k), w(t), w(n), \mathbf{w}:$ Noise. Used in the channel models.

$\mathrm{V}$ : FFS Doppler line based receiver. The coefficients of a receiver using FFS Doppler lines at different delays to compensate both time and frequency dispersion from the channel.

$W:$ Bandwidth.

$\omega, \omega_{0}$ : Relative Doppler frequency. The ratio of the Doppler frequency to the sample frequency multiplied by $2 \pi$.

* : Conjugate of a complex number. 


\section{Abbreviations}

CW

DFE

DPSK

FFT

FIR

FFS

FSK

IFS

IIR

ISI

LMS

LTI

LTV

MAP

MFSK

ML

MLSE

MMSE

PD

PLL

PSK

QPSK

RLS

ROV

SNR

TU-RLS

US

$\mathrm{VCO}$

WHOI

WSSUS

WSS continuous wave

decision feedback equalizer

differential phase shift keying

fast Fourier transform

finite impulse response

finite frequency spread

frequency shift keying

infinite frequency spread

infinite impulse response

inter symbol interference

least mean squares

linear time invariant

linear time variant

maximum aposteriori

multiple frequency shift keying

maximum likelihood

maximum likelihood sequence estimation

minimum mean square error

phase detector

phase locked loop

phase shift keying

quadrature phase shift keying

recursive least squares

remotely operated vehicle

signal to noise ratio

time updated recursive least squares

uncorrelated scattering

voltage controlled oscillator

Woods Hole Oceanographic Institution

wide sense stationary uncorrelated scattering

wide sense stationary 


\section{Chapter 1}

\section{Introduction}

\subsection{Background}

One way of establishing communication between two remote underwater sites is to connect a receiver and a transmitter with a cable. This solution has several disadvantages when one is attempting underwater communication: It is expensive, maintenance and repair is especially difficult if the communication takes place in deep water, and the drag from the cable can be a problem if one of the platforms is small and mobile (e.g., an autonomous vehicle). Another way is to use the water to propagate the signal containing information. Electro-magnetic waves are used for this purpose in air, but they propagate poorly in water, and the attenuation is 40 $\mathrm{dB} / \mathrm{km}$ for light with frequencies in the blue-green region where an attenuation minimum exists [29], [86]. At very low frequencies acoustic waves are able to propagate in the ocean over distances extending to several hundreds of kilometers, and even at $20 \mathrm{kHz}$ the attenuation is only $2-3 \mathrm{~dB} / \mathrm{km}$ and therefore this way of propagating information is chosen. The attenuation of acoustic waves is roughly proportional to the square of the frequency [29], making the communication channel severely band limited. This makes coherent communication more attractive because of its more efficient use of the available bandwidth. 
There has been much work on acoustic wave propagation and modeling of underwater acoustic fields, e.g. [39], [51], and the characteristics in terms of boundary and medium interactions are strongly dependent on the frequency. In order to be able to perform underwater acoustic communication it is important to understand what happens to the information bearing signal on its way from the transmitter to the receiver. Only if this knowledge is in place one can hope to build an efficient and robust communication system. The physics of the signal propagation also plays a key role when one wants to characterize the limitations of a given communication system. Therefore, in order to understand the communication properties of an underwater communication channel, it is important to model the propagation of the acoustic waves in the water at the frequencies used for acoustic communication, and a common way of describing the acoustic sound field is by means of ray theory. This is a high frequency approximation to the sound field, and it is the same as the one used in geometrical optics where the sound is envisioned as arriving over different ray paths. A rough rule for the validity of ray theory is that it applies when the spatial scale of changes in the medium is large compared to the wavelength. In the underwater communication channel this translates to frequencies starting well below $5 \mathrm{kHz}$ and upwards.

The communication channel structure and the obtainable bit rates depend in particular on the range between transmitter and receiver, and the depth of the water. We can sort the communication scenarios into short, medium and long range communication as shown in table 1.1. The table is based on the implemented underwater communication systems reported in the literature over approximately the last 20 years, and thus the figures listed are not theoretical channel capacity measures but rather examples of existing systems.

The short range channel has a dominant direct "line of sight" path. This path is usually very stable and much stronger than the other returns which, depending on the specific communication channel, may be either surface bounce paths or bottom 


\begin{tabular}{|l|l|l|l|}
\hline \hline & Carrier frequency & Bit rates & Range \\
\hline Short range & $>100 \mathrm{kHz}$ & $<1 \mathrm{Mbit} / \mathrm{s}$ & $<1 \mathrm{~km}$ \\
Medium range & $1-100 \mathrm{kHz}$ & $<20 \mathrm{bbit} / \mathrm{s}$ & $1-50 \mathrm{~km}$ \\
Long range & $<1 \mathrm{kHz}$ & $<500 \mathrm{bit} / \mathrm{s}$ & $>50 \mathrm{~km}$ \\
\hline
\end{tabular}

Table 1.1: Classification of different communication channels.

bounce paths or a blend of both.

One characteristic of the medium range channel is that the water depth is less than the range. In the same way as the short range channel this channel has a direct path as well, but boundary interactions are significant. The channel is time-varying and reverberant which means that it has a long impulse response.

In the long range channel the refraction and fluctuation in the ocean is dominant, and there is no direct path; sound is propagating in ducts over ray paths. There is variation induced by the ocean on each ray path, and this causes it to break up into a number of closely propagating rays. It is often called the micro multipath [77], [1], as opposed to the macro multipath consisting of the different ray paths.

The sorting of underwater communication channels according to table 1.1 is somewhat arbitrary. The purpose of the classification is to provide a very general and rough way of recognizing a given scenario. There are many cases where the numbers in table 1.1 are inconsistent with the suggested definitions of short, medium and long range channels. Nevertheless, this way of sorting the channels is useful, and this is motivated by the fact that all the relevant references in this chapter fits in one of the scenarios.

Communication by means of acoustic signals in an underwater environment has proven to be a challenging problem, but the need is demonstrated by the significant number of implementations of acoustic communication systems over the past 20 years. There has to date not emerged as a standard any particular system architecture or modulation scheme. The systems are very different, utilizing most known 
signal processing techniques for communication. We now give an overview of existing communication systems for underwater use, and the systems are sorted in categories according to table 1.1. The overview is by no means exhaustive, but it serves as an indicator of where the emphasis has been in developing these systems.

\subsubsection{Short range, line of sight based systems}

An application is reported in [73] where a data logging platform is telemetering to a surface vessel, i.e., vertical communication. The modulation scheme used is differential phase shift keying (DPSK) and the carrier frequency is $10 \mathrm{kHz}$. By using error correcting codes (BCH, Reed-Solomon) the error probability of $10^{-3}$ for a 6 $\mathrm{km}$ vertical distance is achieved.

A system for transmitting $10 \mathrm{kbit} / \mathrm{sec}$ bursts of data is reported in [48], and in this system the average data rate is $1.5 \mathrm{kbit} / \mathrm{sec}$ over a relatively short channel of $100 \mathrm{~m}$. The modulation used is DPSK, and an array with 16 elements is used to spatially filter the received signal which has a center frequency of $50 \mathrm{kHz}$.

A high frequency very short range system is reported in [56]. It has a carrier frequency of $1 \mathrm{MHz}$, and it is transmitting over a range of $60 \mathrm{~m}$. The achieved data rate is $500 \mathrm{kbit} / \mathrm{sec}$ and the modulation scheme is 16-QAM (quadrature amplitude modulation). In this system an adaptive equalizer is employed to track the channel, and an error rate of $10^{-7}$ is achieved using an LMS equalizer weight adaptation

algorithm. Without the LMS adapted equalizer the error rate is $10^{-4}$. The cause of channel fluctuation is not reported in this reference.

A state of the art vertical communication system is reported in [95], and this is a 4-DPSK system with carrier frequency of $20 \mathrm{kHz}$. It uses compression techniques for transmitting image data from the sea bottom at $6500 \mathrm{~m}$ depth to the surface, and the effective data rate is $16 \mathrm{kbit} / \mathrm{sec}$. In this system the discrete cosine transform is used for image compression, and it is indicated that a compression factor of 12 is achieved on sonar images. 
Communication from sub-bottom positions to a surface vessel is reported in [24], and this is an incoherent system using frequency shift keying (FSK) modulation.

\subsubsection{Medium range, reverberation limited systems}

One of the first systems [17] developed at Woods Hole Oceanographic Institution (WHOI) is an incoherent system using 8-FSK to send information at $4 \mathrm{kbit} / \mathrm{sec}$. The system uses the fast Fourier transform (FFT) to decode the received signal and a Hamming code to make the system more robust. In addition to the information frequencies a continuous waveform (CW) is transmitted to track Doppler shifts.

FSK signaling has proven to be a robust technique in shallow water channels. By using a large alphabet multiple FSK (MFSK) technique communication with 5 $\mathrm{kbit} / \mathrm{sec}$ over a range of $5 \mathrm{~km}$ is reported in [38].

Another system in the same category [20] is used for communication with rate $5 \mathrm{kbit} / \mathrm{sec}$ using $64-\mathrm{FSK}$. The carrier frequency is $20-30 \mathrm{kHz}$, and it is also used successfully for telemetry over such different scenarios as a $4 \mathrm{~km}$ shallow water horizontal path, a $3 \mathrm{~km}$ vertical path and a highly reverberant $700 \mathrm{~m}$ very shallow water path (depth 6-18 m).

A coherent DPSK system based on the direct-sequence spread spectrum technique is reported in [36] where the range is $1 \mathrm{~km}$, the water depth is $10 \mathrm{~m}$, the data rate is $600 \mathrm{bit} / \mathrm{sec}$ and the bandwidth used is $10 \mathrm{kHz}$.

\subsubsection{Long range}

We may use the definition of a long range underwater communication channel as being one where sound propagates in ducts. Then it is clear that some of the medium range channels may turn into long range channels, and this depends on the sound speed profile. When the water temperature or salinity, largely determining the sound speed, changes on a specific site the propagation of sound can easily go from largely 
boundary interacting ray paths to ducted ray paths, therefore some of the systems listed in the previous section also belong in this section.

A system designed solely for long range communication between the mother vessel and several remotely operated vehicles (ROV) is reported in [69]. The ROV's were moving while receiving data, and the maximum speed is 10 knots. This is a low frequency system with carrier frequency $200 \mathrm{~Hz}$ and bandwidth $50 \mathrm{~Hz}$. The system uses a Golay code to increase the reliability, and the modulation scheme is 4-FSK.

Another system for very long range sound propagation (on the order of 1000 $\mathrm{km}$ ) using $\mathrm{m}$-sequences at a carrier frequency of $57 \mathrm{~Hz}$ and a bandwidth of $14 \mathrm{~Hz}$ is reported in [70]. The main purpose of this system is a feasibility demonstration for long range sound propagation and environmental monitoring but the system could also be viewed as a coherent communication system. The propagated signal is an $\mathrm{m}$-sequence [61].

The systems described in [98], [15] demonstrates information transmission over a range of $50 \mathrm{~km}$, where the bit rate is $212.5 \mathrm{bit} / \mathrm{sec}$ and the carrier is $1.7 \mathrm{kHz}$. The modulation is phase shift keying (PSK), and the transmitter is a single element whereas the receiver consists of one array at $150 \mathrm{~m}$ depth and one diversity combiner spanning 100-300 m depth.

\subsubsection{Simulation studies}

A large body of simulation studies is reported in many different periodicals and books. They cover all aspects of underwater acoustic communication systems such as channel identification and tracking, coding, modulation techniques, spatial diversity combining.

Simulations on acoustic channel modeling with emphasis on the communication aspect is given in [28], [33], and this work addressed the stability of the channel multipath and phase.

Channel identification algorithms have also received attention in the former So- 
viet Union, and time delay simulation is reported in [87]. This work is employing bispectra assuming non-Gaussian statistics for signals and noise.

A mode filtering approach is reported in [41]. This is an alternative way of resolving multipath, and the simulation uses vertical line arrays at the transmitter in order to excite a single mode, and at the receiver to accept a single mode. The Pekeris' waveguide is used for this simulation.

The estimation of path time delay is extensively treated $[32,47,66,75,16,59$, $88,2,60,68,42,21,52,49]$, and in some of these studies tracking of the channel is incorporated. An example of a typical approach used to study this problem is given in [72], where the model is deterministic signal in Gaussian nose and the maximum aposteriori estimate of a parameter vector containing amplitudes and delays is computed.

The multichannel receiver for both incoherent [18] and coherent [92] communication is reported to give significant gain, and this work involves both simulations and demonstrations in shallow water environments. The problem of optimally combining multiple channels is also simulated in [102].

Studies and bounds on error probability for various receivers is the important issue in reliability judgments, and bounds in the case of a decision feedback equalizer is reported in [3]. The phenomenon of error propagation is one of the drawbacks for this equalizer [74], [93].

A powerful and general way to deal with low signal to noise ratio (SNR) reverberant channels is various coding techniques, and this is also used in underwater telemetry [19], [79]. Transmission signals made up of $m$-sequences are commonly used because of their statistical properties, and the work in [61] combines coding with the use of $\mathrm{m}$-sequences where both convolutional and block codes are used for error detection and correction.

Emphasis in the literature for underwater acoustic communication is on incoherent reception, and an overview of the existing configurations before 1984 is in [6], 
but also coherent schemes are reported in [92], [91] which comprise both single- and multi- channel results.

\section{Channel tracking}

The adaptive equalizer is widely used to track the time-varying underwater channel, and the combination of beamforming and adaptive equalization is reported in [84] where the equalizer is updated with the LMS algorithm, and ray tracing is used to extract significant paths.

The discussion of the properties of the adaptive algorithms used in channel tracking has received much attention [12], [26]. The two most commonly used are the LMS and recursive least square (RLS) algorithm. The problem of equalization of channels with spectral nulls is treated in [26], and in this reference an alternative recursive algorithm reminiscent of the RLS is used. The improved result is verified with simulations on a time-variant channel.

The adaptive equalization for underwater acoustic telemetry is treated extensively in [78], and here emphasis is on the various algorithms for implementing RLS on decision feedback and maximum likelihood equalizers together with the resulting computational loads.

Summary Modeling of the acoustic propagation is important in underwater communication, and ray theory is a good model at the frequencies of interest in this work. Communication in the ocean is sorted according to short, medium and long range as summarized in table 1.1. Emphasis both in the literature and in the implementation of working systems is on incoherent communication, but coherent communication is also in use. Adaptive systems are used since underwater communication channels are time-variant, and two widely used algorithms are the LMS and the RLS. 


\subsection{The problem of underwater communication}

Preview In this section we discuss in general the main issues of concern in underwater communication channels. We first mention the delay spread which is commonly encountered in many types of communication channels including underwater communication channels. The refraction of sound and the time variance of underwater communication channels caused by ocean internal factors and source/receiver motion are discussed, and one implication of this is Doppler spread. Another characteristic of many underwater communication channels is a sparse impulse response, and we discuss the importance of a receiver that is able to utilize this. An issue in a coherent communication system is the need for synchronization and we outline how this is accommodated. Underwater communication channels are in general doubly spread, and we argue that the Doppler spread becomes increasingly important for lower bit rates. This discussion motivates the importance of the constrained communication problem that we work on in this thesis which is coherent communication over doubly spread channels with more severe Doppler than delay spread, and it is outlined in the next section.

The acoustic signal of underwater communication is in some cases significantly modified by interaction with boundaries, in which case we have a shallow water channel. In shallow water channels the interaction between the acoustic signal and the boundaries (top and bottom) may give delay spread (time dispersion), and then the received signal consists of several delayed and attenuated replicas of the transmitted signal. Delay spread is encountered in many communication channels, e.g., telephone wires, satellite communication, cellular phones, indoor wireless communication. Consequently it has been extensively treated, but it remains an active research area. The underwater communication channel is different from these channels in several aspects, and one important difference is that refraction of the ray paths is a first order effect that can seldom be neglected. Another difference is that 
in the case of surface interacting ray paths there is a time-variant rough reflector present in the communication channel.

The fluctuations in the ocean have many sources, and they are roughly sorted into small scale and large scale phenomena. Factors such as currents, eddies and tidal changes produce large scale fluctuations, and internal waves and turbulence give small scale fluctuations [37]. The impact of different sources of fluctuation is a function of acoustic wave frequency. In addition, at the frequencies in use for communication, the time-varying water surface and the transmitter/receiver motion are sources of time variability of the underwater communication channel. The time variability makes the channel Doppler spread (frequency dispersive), and this is observed through the simple experiment of transmitting a single frequency signal. The received signal from this transmission is amplitude modulated [58], and the received signal spectrum is broader than the transmitted signal spectrum.

The speed and robustness of convergence for any adaptive algorithm, such as least mean squares (LMS) or recursive least squares (RLS), is a function of the number of parameters one is trying to adapt. The number of parameters, or the number of degrees of freedom, impacts the convergence and tracking properties of the adaptive algorithm. By increasing the number of degrees of freedom the robustness degrades, which is seen by the fact that the algorithm is unable to reach any form for meaningful steady state. One important difference between LMS and RLS is , that the convergence speed of LMS depends on the spread of the eigenvalues of the autocorrelation matrix of the received data. High spread yields slow convergence for the LMS, whereas the RLS is not impacted by this. The LMS is described in [103], [45], and the RLS is found in [45], [64]. The latter reference also has a unified treatment of the algorithms. Regardless of the algorithm it is important to maintain good tracking capabilities and this means not to waste degrees of freedom.

A general need for synchronization between the receiver and transmitter is always present, and this is also necessary when performing underwater communication. The 
approach to achieve synchronization has been similar to the one taken in some of the systems for cellular phones, and that is to send a short sequence of known symbols (e.g., Barker sequence), a fixed time before a data packet is transmitted. The receiver constantly performs a matched filter operation to the Barker sequence and uses a threshold test to detect it and decide when a data packet is about to be received.

The underwater communication channel is characterized by its range and Doppler spreading where range translates into delay. The main constraints of our communication problem are available bandwidth, rate of change of the channel and available power. The tradeoff is then often between bit rate, reliability and range. To obtain more reliable communication or communication over a longer range the bit rate may be decreased. The effects of delay and Doppler spread are complementary in the sense that as the bit rate on the communication channel increases, a given delay spread spans more symbols, and this gives more intersymbol interference. When the bit rate decreases the channel variation from one symbol to the next increases so that a given Doppler spread requires better tracking bandwidth in the receiver.

The time interval between two arriving ray paths is often large compared to the symbol duration of the transmitted sequence. Therefore the scattering function of the channel may have clusters of energy widely separated in time, and this is known as a sparse channel. Remembering the need to minimize the number of degrees of freedom this type of channel implies a receiver which is sparse in the sense that it must be able to combine non-contiguous pieces of the received signal.

The bit rates obtained to date in underwater communication channels are relatively modest compared to e.g., satellite communication or cellular phone, and this difference is likely to persist because of the difference in available bandwidth. As pointed out earlier it is the Doppler spread relative to the bit rate that is the important parameter when it comes to channel tracking. At the higher bit rates used in the satellite or cellular phone communication channels the Doppler spread relative to the bit rate is much less than in the underwater communication channel. There- 
fore the problem of communication in presence of Doppler spread has not been as extensively addressed as delay spread in these scenarios.

When the signal is both delay and Doppler spread, we have a doubly spread channel, and this is sometimes the case in underwater communication channels. Doubly spread channels are thoroughly discussed in classical texts on communication [101], [82], [57], but relatively few receivers have been implemented where the channel is assumed to be doubly spread. Rather, one result from the theory of doubly spread channels is heavily used: A channel where the delay-Doppler product is less than one is called underspread, and an underspread channel may be treated as a singly spread channel under certain circumstances. The consequence of this approach is always to sacrifice bit rate to make the channel look singly spread.

Summary Underwater communication channels are time-variant and generally doubly spread, and the Doppler spread is sometimes significant. The fluctuations in the ocean calls for an adaptive communication system, and the sparseness of many of the impulse responses makes it important to use a minimum number of degrees of freedom. The lower bit rates emphasize the importance of compensating Doppler spread.

\subsubsection{A subset of communication channels}

It is shown in subsequent chapters that underwater communication channels are very different depending on propagation conditions. This is also reflected in the fact that among the numerous communication systems implemented there is no prevalent system architecture or modulation scheme. In this work we concentrate on a subset of the observed communication channels. A common feature of the underwater communication channel is that it is sparse, so the subset treated in this work includes sparse channels. As is pointed out above the underwater communication channel has lower obtainable bit rates than some other communication channels such as cellular 
phone or indoor wireless. The consequence of this is that the Doppler spread is more important, and this is further emphasized by the fact that a subset of underwater communication channels exhibit Doppler spread significant relative to the bit rate. Thus the problem discussed in this thesis is identification of physical scenarios with doubly spread channels where the Doppler spread is more severe than the delay spread. Moreover, we are also concerned with how receivers commonly encountered in other communication channels behave in the presence of Doppler spread. We derive and discuss possible solutions that work better on the sparse doubly spread underwater communication channels that have more severe Doppler spread than delay spread.

\subsection{The approach}

Preview In this section we further discuss the problem of communication over doubly spread channels with emphasis on Doppler spread, and we suggest how this is accommodated. We follow an approach that consists of several parts in order to solve the problem of communication over a possibly doubly spread channel. The different parts are channel identification, channel tracking and optimal linear decoding. The acoustic signal is modeled as propagating over a number of rays, and each ray may have a different Doppler shift depending on the ray direction relative to the scatterer velocities. The emphasis is to derive a receiver that works satisfactorily with Doppler spread comprised of different possibly slowly varying Doppler shifts on different ray paths since not much work is reported in this area and it is increasingly important to deal with this distortion as the bit rate decreases.

The first part is concerned with identifying the delay and Doppler spread structure of the channel, and this is carried out by sending a channel probe which is a sequence of data symbols known to both the receiver and transmitter. Then the receiver uses this information to obtain a scattering function estimate, and the quality 
of this estimate depends on the signal to noise ratio and the duration in time and frequency of the data sequence. An important part of this work is to use this part of the receiver without even trying to communicate. If this channel identification procedure is tried in different underwater communication channels it measures the variability of the scattering functions over a wide variety of real ocean channels. This in turn gives a measure of how much delay and Doppler spread one should expect in a given scenario. The aim of the approach in this thesis is not to make a receiver that works well on all channel scattering functions, but rather to look at the subset of channels that are doubly spread with more significant Doppler spread than delay spread. Part of this task is also to simulate the underwater communication channel to verify that the observed spreads can be obtained from reasonable physical mechanisms that we know take place in the ocean.

Given that a reliable estimate of the scattering function of the channel is obtained with the channel probe one would like to incorporate this information in some optimal way for reconstructing the transmitted data sequence. The channel is time-variant and may change during the data sequence transmission, therefore it is necessary to track the channel during data reception. The proposed channel tracker can exploit both the estimated channel structure through its state space description, and can also be used to track changes in the channel by utilizing its recursive way of computing estimates. Therefore, the next part of the receiver is a channel tracker which has embedded in its model the delay-Doppler-spread function for channel characterization and it uses the received data, the transmitted data and its internal model to recursively estimate the delay-Doppler-spread function. The channel tracker always has the received data sequence available, and the first part of the transmitted data i.e, the training set, is a sequence that is known to the receiver. Therefore the channel tracker can make use of the channel input, the channel output and the initially estimated scattering function to obtain initial convergence and tracking of the underwater communication channel. Each snapshot of 
the delay-Doppler-spread function is used by the decoder, to be described below, and the decoder uses the received data and the delay-Doppler-spread function to estimate the nearest allowable data symbol drawn from the alphabet used by the transmitter and known to the receiver, and this is the decoded symbol. When the training sequence is ended, the presumably correctly decoded symbols are used by the channel tracker in place of the training sequence to provide the channel input, and this enables the channel tracker to continue tracking the channel throughout the data packet.

The decoder uses the delay-Doppler-spread function as estimated by the channel tracker, and with this knowledge it optimally combines the different parts of energy that has been dispersed by the channel to estimate the transmitted signal. Varying the optimality criterion gives different decoder structures. The criterion adopted in this work is optimization with respect to the minimum mean square error between the estimated and true data symbol.

The receiver/transmitter synchronization is obtained by means of a Barker sequence, and the receiver continuously performs a matched filter operation to this sequence. When a packet start is detected by means of the matched filter output exceeding a threshold, the scattering function estimate starts after a fixed delay.

To verify the receiver capability it is used on real and simulated data and compared with a different receiver structure that is currently in use. A summary of the receiver built up by this approach is shown in Fig. 1-1. 


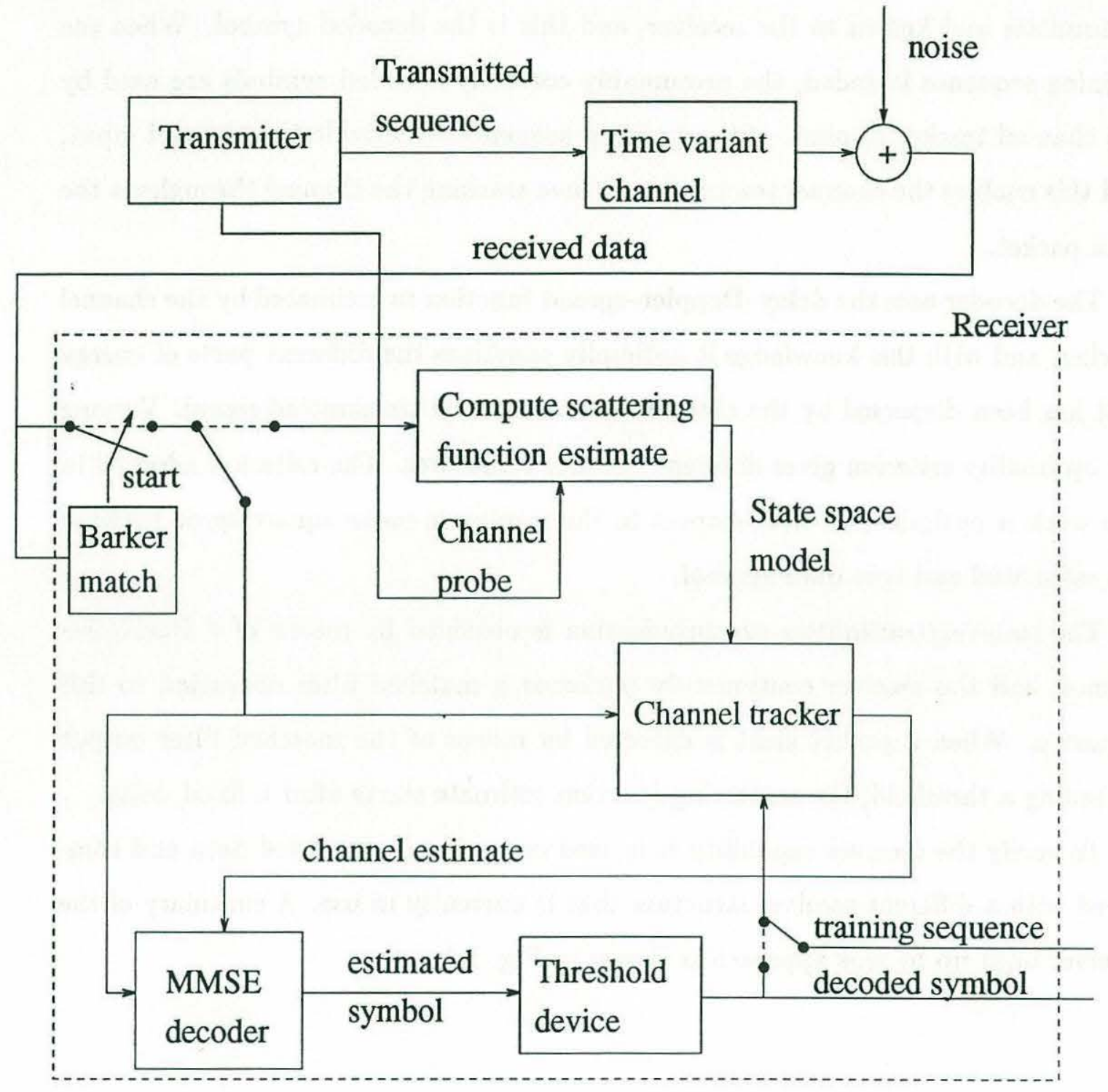

Figure 1-1: An overview of the proposed system for use in the underwater communication channel. 


\subsection{Thesis overview}

The thesis is organized according to the approach outlined above. Chapter 2 gives the necessary definitions and introduces the theoretical framework for characterization of the time-variant channels. It introduces and discusses the scattering function and relates this channel characterization tool to the ambiguity function commonly used in sonar and radar, and also to a function used for characterizing linear timevariant (LTV) channels called the delay-Doppler-spread function. Another function commonly used for characterization of LTV channels called the input delay-spread function is also presented and discussed in this chapter. The random behavior of the underwater communication channel makes it convenient to use a statistical description of the LTV. The concept of a wide sense stationary uncorrelated scattering (WSSUS) channel is central to the development of this description and also to the formulation of the channel tracker. Therefore this chapter introduces and discusses the WSSUS channel. The work in this chapter is not my own, but rather a compilation of pieces of work by various other authors [7], [101], [82], [83].

As discussed in the introduction of this chapter, the understanding and modeling of the acoustic wave propagation is essential in the understanding of how to make robust and efficient use of the underwater communication channel. Therefore, Chapter 3 develops a simulation tool based upon a raytrace representation of the acoustic field and a time-variant finite impulse response (FIR) filter, and it gives the connection between observed Doppler and delay spread and the physical processes in the ocean. Herein, is also contained the estimated scattering functions of a few very dif-

ferent underwater communication channels motivating the imposed constraint in the problem formulation to deal with a subset of underwater communication channels.

Given the channel model developed in Chapter 2 and the verification of its relevance in Chapter 3 we derive the maximum likelihood (ML) receiver structure based on this channel model in Chapter 4. The doubly spread channel is a generalization 
containing both the time-invariant delay spread channel and the purely Doppler spread channel, and the receivers for these channels are shown to be special cases of the doubly spread receiver. The ML receiver for a doubly spread channel gives a high computational load, therefore we resort to the suboptimal minimum mean square er-

ror (MMSE) receiver outlined above. The main concern is on Doppler spread, and a common nonlinear receiver called the decision feedback equalizer (DFE) is analyzed in particular with respect to a Doppler spread signal. The verification and evaluation of the receiver design is carried out by testing it on both data obtained from the simulator described in Chapter 3, and also on data acquired from the ocean.

Finally in Chapter 5 some conclusions and future directions are outlined.

\section{Thesis contributions}

The contributions of this thesis are roughly divided in three parts:

1 The first part is on underwater communication channel identification. The measurements and scattering function estimates computed from a large number of transmissions varying geographically from the Arctic, ice covered ocean to tropical waters bring out clearly that there is no such thing as "the" underwater communication channel. The characteristics in terms of delay and Doppler spread are so different that one can hardly hope for one particular communication system serving all these channels appropriately. An important result of the channel measurements is to highlight the presence and importance of Doppler spread in some of the channels. This spread is so far in the literature usually attributed to the "rapidly varying" nature of the ocean. The transmitter/receiver motion and the physical ocean processes producing time variability such as waves or currents are not taken into account, therefore the variability is modeled with random processes. It is shown in this thesis that some of the variation originates from different Doppler shifts on different ray paths or a time-variant Doppler on some of the ray paths. This is supported by 
acoustic measurements from the ocean combined with information about geometry, sound speed, surface conditions, etc. Moreover, through a simulator the explanation for some of the observed Doppler spread is linked to physical processes that we know take place in the ocean such as transmitter and receiver motion and surface waves. This serves as a justification for the adopted explanation of the Doppler spread.

2 The second part is the analysis of the commonly used RLS algorithm with respect to Doppler spread. The analysis gives insight into the behavior of a receiver adapted with RLS during the reception of a Doppler spread signal, and this behavior is correctly predicting the result when running on real data. When a phase locked loop (PLL) is employed to take care of the Doppler spread through tracking of the instantaneous frequency the analysis also brings out clearly the interaction between the two devices (the RLS algorithm and the PLL) and shows that the result is an ill-posed system. Specifically, some amount of the Doppler spread is compensated by the RLS and this produces tap rotation. As a result of this the RLS updating the taps is required to have large bandwidth because the PLL compensates the Doppler spread insufficiently. Related work and results are found in the literature, and is referenced at the relevant locations, but the detailed analysis of the composite system of the PLL and the RLS algorithm has not been found anywhere else. For simple Doppler shifts a good compensation is achieved by estimating the shift from the cross-ambiguity function and applying phase rotation of the signal before it enters the receiver.

3 The third part is a contribution towards developing a receiver that works on a sparse doubly spread channel with emphasis on using a minimum number of degrees of freedom. The model for the channel tracker in this receiver is motivated by the channel model developed and verified through real data measurements and simulations, and the insight gained from the analysis of the RLS algorithm is incorporated in this channel tracker. A modified RLS algorithm with a time update step 
incorporating the knowledge about Doppler is presented. This algorithm, called the time updated RLS (TU-RLS), is applied in the channel tracker that estimates the cross-ambiguity function of the channel. This estimate is used in a decoder that employs Doppler lines, which are frequency domain filters replacing the PLL, and the decoded data are used by the channel tracker to maintain the cross-ambiguity function estimate. This receiver configuration has neither been encountered in the literature that has been surveyed on underwater communication nor in any other communication channel, and it represents an attempt to deal with doubly spread channels without implicitly degenerating them to singly spread channels at the expense of bit rate [101]. The operational capability of the receiver is verified on both simulated and real data. An experiment was performed in shallow water near Newport RI during February 1996. Some of the data collected in this experiment is severely Doppler spread, and efforts to decode this data with already existing receivers have been unsuccessful. The data are successfully decoded with one of the receivers derived in this thesis! 


\section{Chapter 2}

\section{Characterization of time-variant}

\section{channels}

\subsection{Preview and motivation}

For reasons that were pointed out in Chapter 1 the underwater communication channel is modeled as an LTV system, and the purpose of this chapter is to present appropriate mathematical tools to analyze and work with these systems. The class of all LTV systems is very large and, for the purpose of the modeling of underwater communication channels, we are interested in only a subset. The physics of the underwater communication channel helps us to identify the subclass that models the underwater communication channel well. The main constraint is the WSSUS assumption that is discussed in conjunction with (2.4). We know from acoustical modeling to be described in Chapter 3 that the channel can be thought of in various ways. One representation is as time-variant scatterers at different delays, and this gives the input delay-spread function in (2.1). Another representation is as constant in time scatterers at different delays moving at different speeds, and this gives the delay-Doppler-spread function in (2.2).

The WSSUS assumption allows us to define the channel scattering function which 
is a two dimensional power spectral density in delay and Doppler, and this function is defined in (2.7). The scattering function could be directly computed from the complete statistical description of the delay-Doppler-spread function, but this knowledge is seldom available. In a practical experiment we can not hope for much more than a characterization in terms of the second order statistics of the channel which are given by the scattering function. Thus to get the second order statistics we need a way to estimate the scattering function, and this can be found from the cross-ambiguity function as shown in (2.34).

Delay spread and Doppler spread are distortions introduced by the underwater communication channel in time and frequency, respectively. When we want to communicate over this channel we need to understand and take into account both of these dispersions. In Section 2.4 on Doppler lines and delay lines we make connections between purely delay spread and purely Doppler spread channels. The concept of duality is utilized to draw on knowledge about the more common delay lines and transfer this to the less common Doppler lines. The Doppler lines play a similar role for modeling and equalizing Doppler spread channels as do the delay lines for delay spread channels, and this is illustrated in (2.62) and (2.66). Both the delay lines and Doppler lines are linear devices which can be characterized by their eigenfunctions, and this is performed in the discussion surrounding (2.73).

\subsection{Wide sense stationary uncorrelated scatter- ing channel}

The wide sense stationary uncorrelated scattering (WSSUS) assumption is essential to much work reported in the literature on LTV systems, some examples where this is implicitly or explicitly used are [82], [101], [44], [4], [63], [55]. The WSSUS assumption is embedded in the receiver that is derived later in this work, and we also need to invoke this assumption in order to make connections between the cross- 
ambiguity function, the channel scattering function and the delay-Doppler-spread function all to be introduced in this chapter. All of these functions are useful for the channel identification work that we present in Chapter 3. The framework for the WSSUS assumption is developed in [7], [8], and we give a summary of some of the conclusions therein. In order to explain some of the consequences of the WSSUS assumption we introduce some useful functions for characterizing LTV systems.

There are many equivalent ways of characterizing LTV systems, and [7] contains a thorough analysis of eight related system functions that can be used to describe LTV systems. It establishes connections between these and four other system functions introduced elsewhere [54], [107]. These system functions are commonly used in other areas where LTV systems occur and are sometimes referred to as the "Bellofunctions" [89]. We do not present an exhaustive discussion of these functions, but introduce some of those that give insight into our problem. One main difference between the various functions is the connection they have to distinct physical models. The input delay-spread function $h(t, \tau)$ relates the input $x(t)$ to the output $y(t)$ of the LTV by

$$
y(t)=\int x(t-\tau) h(t, \tau) d \tau .
$$

This equation can physically be depicted as a continuum of non-moving scintillating scatterers. Thus the physical interpretation in the context of an underwater communication channel is that we have scattering from all the volume and boundaries that are insonified by the sound, but the scattering strengths from different parts of the volume and boundaries vary with time. Another useful function in our case is the delay-Doppler-spread function given by

$$
y(t)=\iint x(t-\tau) U(\tau, \nu) e^{j 2 \pi \nu t} d \tau d \nu
$$

where the physical equivalent is a channel with scatterers moving at different veloc- 
ities and at different locations (delays spread function and the delay-Doppler and it is given by

\section{Health Education at MIT Medical}

253-1316, web.mit.edu/medical/healthed

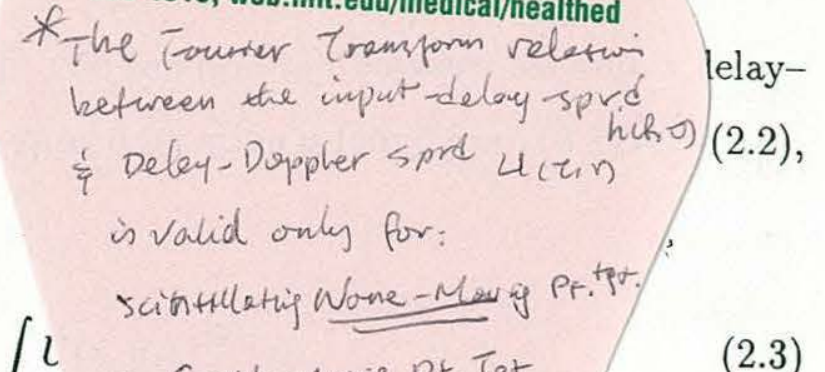

$$
h(t, \tau)=\int \iota
$$

Thus one interpretation of the delay-Dopple

composition in the time variable of the input delay-spreau sunction. $U(\tau, \nu)$ is the complex scattering amplitude of the scatterers within the delay $(\tau, \tau+d \tau)$ and Doppler $(\nu, \nu+d \nu)$. A useful physical interpretation of this channel representation is that the scattering strength of each scatterer is not changing in time, because $U(\tau, \nu)$ itself is not a function of time, and the time-variance of the channel enters only through the fact that the scatterers are moving. The presence of a Doppler shift due to a moving scatterer means, by definition, that the delay is also changing. Thus the physical interpretation is not in general valid but, as the following numbers show, it is useful in our scenario. A typical symbol rate is $1000 \mathrm{symb} / \mathrm{sec}$ so that a symbol extends $1500 / 1000 \mathrm{~m}=1.5 \mathrm{~m}$ when the sound speed is $1500 \mathrm{~m} / \mathrm{sec}$. A Doppler shift of $5 \mathrm{~Hz}$ at $20 \mathrm{kHz}$ carrier means a scatterer speed of $0.38 \mathrm{~m} / \mathrm{sec}$. We transmit data in packets of typical length $2 \mathrm{sec}$, and the scatterer moves $0.38 \times 2 \mathrm{~m}=0.76 \mathrm{~m}$ in this time. Thus the scatterer is within one symbol length $(=1.5 \mathrm{~m})$ during the entire transmission. This is the assumption that makes the physical interpretation above useful, and it should be used with care since it is obviously broken for higher Doppler, higher symbol rate or longer packet length. Thus we may think of $U(\tau, \nu)$ as a short term stationary model.

We allow $U(\tau, \nu)$ to be a time-dependent function $U(\tau, \nu ; t)$ in later chapters, but in order for this model to be useful we assume that the change in $U(\tau, \nu)$ is much slower than the time variance caused by the scatterer motion in accordance with the example above. If this assumption is violated the model looses its significance. 
There is no need to factor out $e^{j 2 \pi \nu t}$ in $(2.2)$ if $U(\tau, \nu ; t)$ varies at the same rate as $e^{j 2 \pi \nu t}$. The assumption of slowly varying delay-Doppler-spread function is used in the derivation of the receiver in Chapter 4.

Now let us assume that the channel is wide sense stationary (WSS), and by that we mean that $h(t, \xi)$ is a WSS random process with respect to its time variable $t$ so that

$$
R_{h}(t, t-\tau ; \eta, \xi)=E\left[h(t, \eta) h^{*}(t-\tau, \xi)\right]=R_{h}(\tau ; \eta, \xi) .
$$

The absolute time $t$ when we excite the channel is irrelevant to the computation of $R_{h}$ in (2.4), and the WSS assumption implies that $R_{h}(t, t-\tau ; \eta, \xi)$ is not a function of $t$.

In addition we assume that the channel is made up of uncorrelated scatterers, and by that we mean that no matter how close two scatterers are in the channel they produce uncorrelated scattering. This means that $h(t, \xi)$ is a random process that is uncorrelated in its delay variable $\xi$, and it is called the uncorrelated scattering (US) assumption:

$$
R_{h}(\tau ; \eta, \xi)=P_{h}(\tau, \xi) \delta(\eta-\xi)
$$

where

$$
P_{h}(\tau, \xi)=E\left[h(t, \xi) h^{*}(t-\tau, \xi)\right] .
$$

In the case of the delay-Doppler-spread function the WSSUS assumption will give a particularly simple form of the autocorrelation function:

$$
R_{U}(\xi, \eta ; \nu, \mu)=E\left[U(\xi, \nu) U^{*}(\eta, \mu)\right]=S(\xi, \nu) \delta(\mu-\nu) \delta(\eta-\xi)
$$


where $S(\xi, \nu)$ is the channel scattering function which is a two dimensional power spectrum density in delay and Doppler. Equation (2.7) can be derived by using the WSS assumption to get impulsive behavior in the Doppler variable and the US assumption get impulsive behavior in the delay variable of $R_{U}(\xi, \eta ; \nu, \mu)$. The corresponding physical interpretation of the WSSUS channel is that it may be represented as a collection of uncorrelated non-scintillating scatterers which cause both delay and Doppler shift. The channel scattering function $S(\xi, \nu)$ can be derived from $h(t, \xi)$ and other LTV representations as well, but it is defined if and only if the WSSUS channel assumption is adopted, and in this case it gives exhaustive information about the second order statistics of the channel. Therefore $S(\xi, \nu)$ is an essential parameter when characterizing the underwater communication channel, and we need to obtain estimates of it.

In a regular experiment the only accessible data is the input and output of the channel, and we are not likely to have direct measurements of any of the quantities $U(\tau, \nu)$ or $h(t, \xi)$. Thus we present a function that will be useful for the purpose of estimating $S(\xi, \nu)$ from input and output channel data only, and it is called the crossambiguity function. In Section 2.3.1 we also show that different physical mechanisms for Doppler spread may generate similar cross-ambiguity functions, and we comment on the narrowband assumption inherent in the development that leads to (2.32) in Section 2.3.2. For this we need a convenient representation of the transmitted data sequence, and we now present the complex envelope of a signal which is a common way of representing narrowband signals.

\subsubsection{Representation of the transmit signal}

The sequence of information symbols is mapped into a continuous time waveform suitable for transmission over the underwater communication channel, and we now discuss how this can be carried out. For this purpose we define the real valued 
continuous time passband signal

$$
x_{0}(t)=A(t) \cos \left(2 \pi f_{c} t+\phi(t)\right)
$$

where $f_{c}$ is the carrier frequency which is in the range $5-50 \mathrm{kHz}$ for the data shown in Chapter 3. The quantity $\phi(t)$ is the phase of $x_{0}(t)$ which is used to carry the information in the phase coherent systems considered here. The variation of $\phi(t)$ is such that the frequency content of $x_{0}(t)$ is concentrated in a narrow band around $f_{c}$. The real signal $A(t)$ is the amplitude envelope of $x_{0}(t)$, and it can be, e.g., a train of rectangular pulses or a train of raised cosines. Let us assume that it is given by a rectangular pulse train

$$
A(t)=1 / \operatorname{Trect}(t)
$$

where

$$
\operatorname{rect}(t)= \begin{cases}1 & \text { for } 0<t<T \\ 0 & \text { elsewhere }\end{cases}
$$

If we expand (2.8) we get another representation for the transmitted signal $x_{0}(t)$ :

$$
x_{0}(t)=[A(t) \cos \phi(t)] \cos \left(2 \pi f_{c} t\right)-[A(t) \sin \phi(t)] \sin \left(2 \pi f_{c} t\right)
$$

where the two terms in the brackets are the quadrature components. This expression is also conveniently written as

$$
x_{0}(t)=\operatorname{Re}\left[A(t) e^{j \phi(t)} e^{j 2 \pi f_{c} t}\right]=\operatorname{Re}\left[x(t) e^{j 2 \pi f_{c} t}\right]
$$

where we have introduced the complex envelope $x(t)=A(t) e^{j \phi(t)}$ which is a lowpass signal centered around $0 \mathrm{~Hz}$. We use QPSK modulation, so the information sequence 
phase $\phi(t)$ is constant over the symbol period, and we write

$$
\phi(t)=\phi(n)(n-1) T \leq t<n T
$$

where

$$
\phi(n) \in\left\{-\pi,-\frac{\pi}{2}, 0, \frac{\pi}{2}\right\}
$$

The transmitted signal is written in terms of the complex envelope as

$$
\begin{aligned}
\sum_{n} x(t-n T) & =1 / T \sum_{n} A(t-n T) e^{j \phi(t-n T)}=1 / T \sum_{n} \operatorname{rect}(t-n T) e^{j \phi(n)} \\
& =1 / T \sum_{n} z(n) \operatorname{rect}(t-n T)
\end{aligned}
$$

where

$$
z(n) \in\{-1,-j, 1, j\}
$$

is the information symbol sequence. The three representations (2.8), (2.11) and (2.12) are all equivalent, and we use the complex envelope in (2.12). In order to recover the information from $x(t)$ we proceed as shown in Fig. 2-1. The multiplier

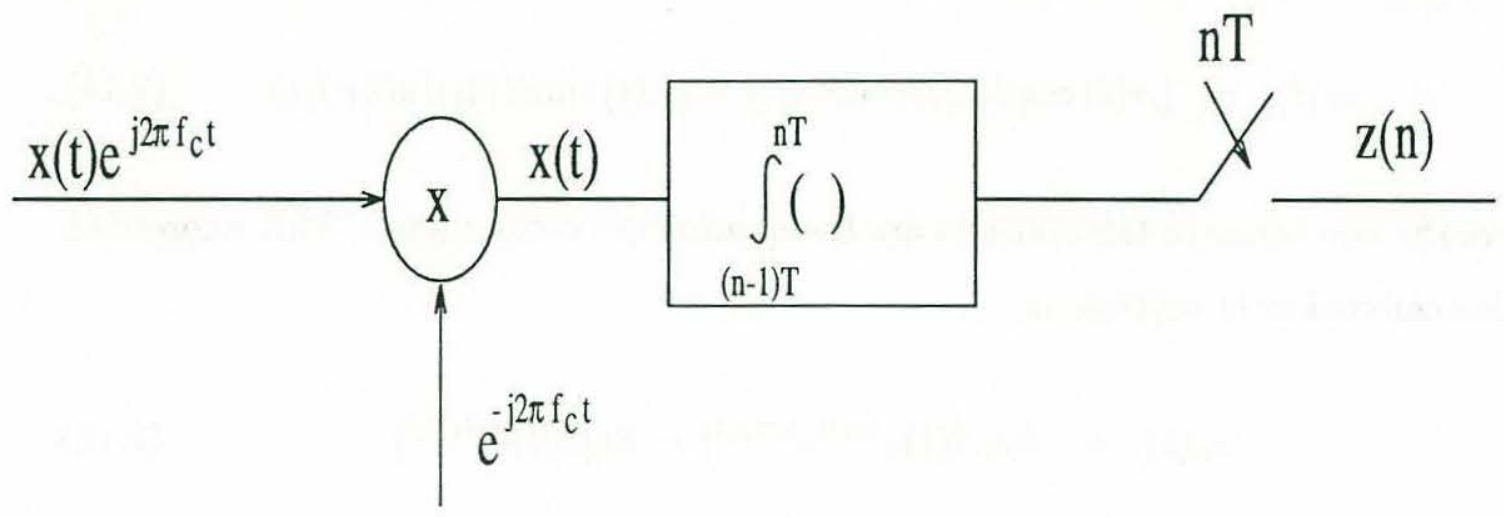

Figure 2-1: Recovering the information sequence from the carrier modulated complex envelope. 
to the left in Fig. 2-1 demodulates the received signal, and the integrator acts as a matched filter to the shaping pulse $A(t)$. The real valued signal $x_{0}(t)$ that is transmitted has phase shifts of $n \pi / 2$ for $n \in\{0,1,2,3\}$ every symbol period, and this information is contained in the complex sequence $z(n)$. Implicit in the interpretation of Fig. 2-1 is the quadrature demodulator. This means that we multiply the received signal $x_{0}(t)$ with the two sinusoids $\cos \left(2 \pi f_{c} t\right)$ and $\sin \left(2 \pi f_{c} t\right)$ and, as is seen

from (2.11) and (2.12), the result is represented as the complex signal $x(t)$. The introduction of $x(t)$ is merely a convenient notation that is often used, and for this reason we adopt it in this work.

We now have a representation of the transmitted signal that explicitly contains the sequence of information symbols, and we proceed with the discussion of the cross-ambiguity function.

\subsection{Ambiguity function}

The ambiguity function is often used in radar and sonar to characterize transmit waveforms, and in particular how the transmit waveform affects the estimation of the range and velocity of a point reflector. We follow closely the derivation in [101], and we define the complex envelope of the transmitted signal to be $x(t)$. The relation between $x(t)$ and the transmitted signal is

$$
x_{0}(t)=\sqrt{\mathcal{E}} \operatorname{Re}\left[x(t) e^{j 2 \pi f_{c} t}\right]
$$

where $f_{c}$ is carrier frequency. The transmitted signal has energy $\mathcal{E}$, and $x(t)$ is normalized according to

$$
\int_{-\infty}^{\infty}|x(t)|^{2} d t=1
$$


If there is a reflector at delay $\tau$ moving to give Doppler shift $\nu_{d}$, we model the complex envelope of the received signal as

$$
y(t)=\sqrt{\mathcal{E}} \dot{b} x(t-\tau) e^{j 2 \pi \nu_{d} t}+w(t)
$$

where $w(t)$ is complex Gaussian circular [97] white noise with covariance

$$
R(t, u)=N_{0} \delta(t-u)
$$

and $b$ is a zero-mean, complex Gaussian random variable representing the reflection process. $\tau$ and $\nu_{d}$ are unknown parameters for which we want to obtain maximum likelihood (ML) estimates. Thus we form the sufficient statistic

$$
L\left(\hat{\tau}, \hat{\nu}_{d}\right)=\int y(t) x^{*}(t-\hat{\tau}) e^{-j 2 \pi \hat{\nu}_{d} t} d t .
$$

If $\left(\hat{\tau}, \hat{\nu}_{d}\right)$ took on a finite number of values we would have a multi-hypothesis problem where the solution would be a set of tests involving likelihood ratios [101], [82]. In our problem $\left(\hat{\tau}, \hat{\nu}_{d}\right)$ take on a continuum of values, and we have a parameter estimation problem. In this case we form the $\log$ likelihood function $\ln \Lambda$ which is proportional to the magnitude squared of the sufficient statistic. Thus we look for the maximum of the likelihood function

$$
\ln \Lambda\left(\hat{\tau}, \hat{\nu}_{d}\right) \sim\left|L\left(\hat{\tau}, \hat{\nu}_{d}\right)\right|^{2}
$$

as a function of $\hat{\tau}$ and $\hat{\nu}_{d}$. Then (2.19) and (2.21) give

$$
\begin{aligned}
L\left(\hat{\tau}, \hat{\nu}_{d}\right) & =\sqrt{\mathcal{E}} b \int x(t-\tau) x^{*}(t-\hat{\tau}) e^{j 2 \pi\left(\nu_{d}-\hat{\nu}_{d}\right) t} d t \\
& +\int w(t) x^{*}(t-\hat{\tau}) e^{-j 2 \pi \hat{\nu}_{d} t} d t .
\end{aligned}
$$


By defining

$$
\begin{aligned}
\tau^{\prime} & =\hat{\tau}-\tau \\
\nu_{d}^{\prime} & =\hat{\nu}_{d}-\nu_{d} \\
n\left(\hat{\tau}, \hat{\nu}_{d}\right) & =\int w(t) x^{*}(t-\hat{\tau}) e^{-j 2 \pi \hat{\nu}_{d} t} d t
\end{aligned}
$$

we get the likelihood from (2.22) and (2.23) as

$$
\begin{aligned}
\ln \Lambda\left(\hat{\tau}, \hat{\nu}_{d}\right) & =\mathcal{E}|b|^{2}\left|\int x(t-\tau) x^{*}\left(t-\tau-\tau^{\prime}\right) e^{-j 2 \pi \nu_{d}^{\prime} t} d t\right|^{2} \\
& +2 \sqrt{\mathcal{E}} \operatorname{Re}\left[b n^{*}\left(\hat{\tau}, \hat{\nu}_{d}\right) \int x^{*}(t-\tau) x\left(t-\tau-\tau^{\prime}\right) e^{-j 2 \pi \nu_{d}^{\prime} t} d t\right] \\
& +\left|n\left(\hat{\tau}, \hat{\nu}_{d}\right)\right|^{2} .
\end{aligned}
$$

In the absence of noise, the output of the ML receiver for $\left(\tau, \nu_{d}\right)$ scaled by $\mathcal{E}|b|^{2}$ is

$$
\theta_{0}\left(\tau^{\prime}, \nu_{d}^{\prime}\right)=\left|\int x\left(z+\tau^{\prime} / 2\right) x^{*}\left(z-\tau^{\prime} / 2\right) e^{-j 2 \pi \nu_{d}^{\prime} z} d z\right|^{2}
$$

where we have made the substitution $z=t-\tau-\tau^{\prime} / 2$ to emphasize that $\theta_{0}\left(\tau^{\prime}, \nu_{d}^{\prime}\right)$ is a function of $\tau$ and $\nu_{d}$ only through the differences $\left(\tau^{\prime}, \nu_{d}^{\prime}\right)$. This is a measure of the degree of similarity between the complex envelope and a replica shifted in time and frequency, and it is known as the ambiguity function [101], [106]. The likelihood function in (2.25) and also the cross-ambiguity function to be introduced in this section are random variables. An important measure of their performance is the ratio of their mean to standard deviation. This is discussed in Section 4.3.6 and Appendix C.

The ambiguity function is used to characterize transmit waveforms with respect to their ability to detect and estimate the range and speed of moving point reflectors. In this work we are interested in characterizing communication channels, therefore we develop the concept of the ambiguity function a little further in this section. 
We want to introduce the cross-ambiguity function which is a channel identification tool that is used with time-variant channels. For this purpose we make a parallel relation that shows the connection between the cross-correlation function and the identification of time-invariant systems. Let us consider the channel identification problem of Fig. 2-2, and let the system $h(\tau)$ and thus $\hat{h}(\tau)$ be linear time-invariant (LTI). Assume that the noise $w(t)$ is uncorrelated with $x(t)$ and that we want to find

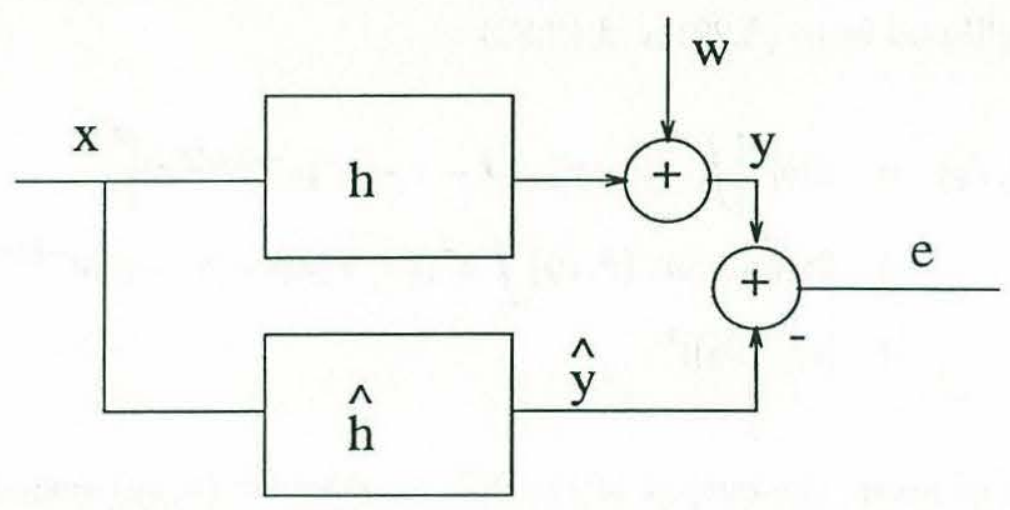

Figure 2-2: Channel identification problem, no inversion needed because both channel input and output are known.

$\hat{h}(\tau)$ so that $E\left[|y(t)-\hat{y}(t)|^{2}\right]$ is minimized. $x(t)$ is a WSS process with covariance $R_{x x}(\tau)$, and we have that

$$
\begin{aligned}
& y(t)=\int h(\tau) x(t-\tau) d \tau+w(t) \\
& \hat{y}(t)=\int \hat{h}(\tau) x(t-\tau) d \tau
\end{aligned}
$$

The minimization of $E\left[|y(t)-\hat{y}(t)|^{2}\right]$ over $\hat{h}(\tau)$ is a well known problem and the solution can be found in [101]. Omitting the derivation we have that $\hat{h}(\tau)$ is given by

$$
R_{x y}(\tau)=\int R_{x x}(\tau-s) \hat{h}(s) d s
$$


where

$$
\begin{aligned}
& R_{x y}(\tau)=E\left[x(t) y^{*}(t-\tau)\right] \\
& R_{x x}(\tau)=E\left[x(t) x^{*}(t-\tau)\right] .
\end{aligned}
$$

If $x(t)$ is a white process the covariance $R_{x x}(\tau)$ of $x(t)$ is given by

$$
R_{x x}(\tau)=\sigma_{x}^{2} \delta(\tau)
$$

and if $\sigma_{x}^{2}=1$ we get from (2.28)

$$
\hat{h}(\tau)=R_{x y}(\tau)
$$

This is the parallel relation that can be used to motivate a generalization of the ambiguity function. We now use (2.26) to construct a new function that can be used for channel identification on a time-variant channel in a similar way as the cross-correlation (2.31) is used in the time-invariant channel. Consider the quantity

$$
\theta\left(\tau, \nu_{d}\right)=\left|\int x(t+\tau / 2) y^{*}(t-\tau / 2) e^{-j 2 \pi \nu_{d} t} d t\right|^{2}
$$

which we call the cross-ambiguity function [10]. Note that in the case of $\nu_{d}=0$ we have the squared magnitude of a cross correlation estimate between $x$ and $y$ on the right hand side of (2.32). The purpose of presenting the derivation (2.27)-(2.31) is to make a connection between (2.32) and (2.26). The expression (2.31) shows how the channel in an LTI system can be identified from the knowledge of the input and output signal only. This suggests that $\theta\left(\tau, \nu_{d}\right)$ is an estimate of the channel response in the case of an LTV system.

The ambiguity function in (2.26) is introduced by means of a parameter estimation problem where we want to find $\left(\hat{\tau}, \hat{\nu}_{d}\right)$. The cross-ambiguity function is 
introduced by means of the system identification problem in (2.31), where we want to find $h(\tau)$. We note that these two problems are related, since part of a system identification problem is to find the delays $\tau$. The quantity inside the squared magnitude of (2.32) is called the time-frequency correlation function, and this is a more complete analogy to the system identification problem (2.31).

The cross-ambiguity function uses only the input and output data from the channel, and we now show its relation to the channel scattering function that was introduced in (2.7). By this derivation we demonstrate that there is a relationship similar to (2.31) of the case of an LTV system, and for this purpose we consider the noise free case of (2.2). We return to the general case in Section 4.3.6. By inserting $(2.2)$ in $(2.32)$ we get

$$
\begin{aligned}
& \theta\left(\tau, \nu_{d}\right)=\int \cdots \int x\left(t_{1}+\tau / 2\right) x^{*}\left(t_{1}-\tau / 2-\xi_{1}\right) U^{*}\left(\nu_{1}, \xi_{1}\right) e^{-j 2 \pi\left(\nu_{1}+\nu_{d}\right) t_{1}} \\
& x^{*}\left(t_{2}+\tau / 2\right) x\left(t_{2}-\tau / 2-\xi_{2}\right) U\left(\nu_{2}, \xi_{2}\right) e^{j 2 \pi\left(\nu_{2}+\nu_{d}\right) t_{2}} d t_{1} d t_{2} d \nu_{1} d \nu_{2} d \xi_{1} d \xi_{2}
\end{aligned}
$$

where we have just written out the magnitude squared in (2.32) as two nested conjugate integrals. We now take the expectation of this equation, and note that by means of (2.7) we can integrate over $\nu_{2}$ and $\xi_{2}$ to get

$$
\begin{aligned}
E\left[\theta\left(\tau, \nu_{d}\right)\right]= & \iiint \int x\left(t_{1}+\tau / 2\right) x^{*}\left(t_{1}-\tau / 2-\xi_{1}\right) \\
& x^{*}\left(t_{2}+\tau / 2\right) x\left(t_{2}-\tau / 2-\xi_{1}\right) S\left(\nu_{1}, \xi_{1}\right) e^{-j 2 \pi\left(\nu_{d}+\nu_{1}\right)\left(t_{1}-t_{2}\right)} d t_{1} d t_{2} d \nu_{1} d \xi_{1} \\
= & \iint\left|\int x\left(t_{1}^{\prime}+\frac{\tau+\xi}{2}\right) x^{*}\left(t_{1}^{\prime}-\frac{\tau+\xi}{2}\right) e^{-j 2 \pi\left(\nu_{d}+\nu_{1}\right) t_{1}^{\prime}} d t_{1}^{\prime}\right|^{2} S\left(\nu_{1}, \xi_{1}\right) d \nu_{1} d \xi_{1} \\
= & \iint \theta_{0}\left(\tau+\xi_{1}, \nu_{d}+\nu_{1}\right) S\left(\xi_{1}, \nu_{1}\right) d \xi_{1} d \nu_{1}
\end{aligned}
$$

where we use the variable substitution $t_{1}=t_{1}^{\prime}+\xi_{1} / 2, t_{2}=t_{2}^{\prime}+\xi_{1} / 2$ and also use (2.26) to arrive at the last expression. The physical interpretation of this is that by computing the expectation of the cross-ambiguity function we view the true channel scattering function through a convolution with the signal ambiguity function [101]. 
Therefore in order to get a best possible estimate of the channel scattering function the ambiguity function of the signal should be as impulsive as possible in both delay and Doppler, and this translates into the desire for a wide band signal of long duration [101]. In this reference there is also a list of standard properties of the normalized ambiguity function, and one of these is the normalization property $\theta(0,0)=1$. For mathematical brevity we use unnormalized cross-ambiguity functions in Chapter 4 because it is the relative shape of this function that is important. In particular we have that if

$$
\theta_{0}\left(\tau, \nu_{d}\right)=\delta(\tau) \delta\left(\nu_{d}\right)
$$

then (2.34) yields

$$
E\left[\theta\left(\tau, \nu_{d}\right)\right]=S\left(\tau, \nu_{d}\right)
$$

and this further illustrate the relationship (2.34).

\subsubsection{Time-variant and multiple Doppler shifts}

The cross-ambiguity function can be interpreted as an estimate of the scattering function as was shown in (2.7) and (2.34), and one assumption is that the delayDoppler-spread function is time-independent so that the strength and Doppler shift on each ray are constant vs time. This may be violated if the integration time $T$ in the cross-ambiguity function increases. In the models for the receivers discussed in Section 4.3 the delay-Doppler-spread function is allowed to be slowly time varying. It is important when interpreting the cross-ambiguity function to understand that there may be different physical mechanisms generating the same shape of the crossambiguity function. We are in particular concerned with Doppler spread, and we now show an example of how different scenarios can give the same shape of the cross- 
ambiguity function. In Fig. 2-3 is shown a simulated received signal with rectangular pulse shape arriving over a single ray path. The Doppler shift $\nu$ of this ray is a function of time and is varying more than $3 \mathrm{~Hz}$ over the time window of roughly 2 sec shown in the upper panel. This takes place if the ray interacts with an ocean-
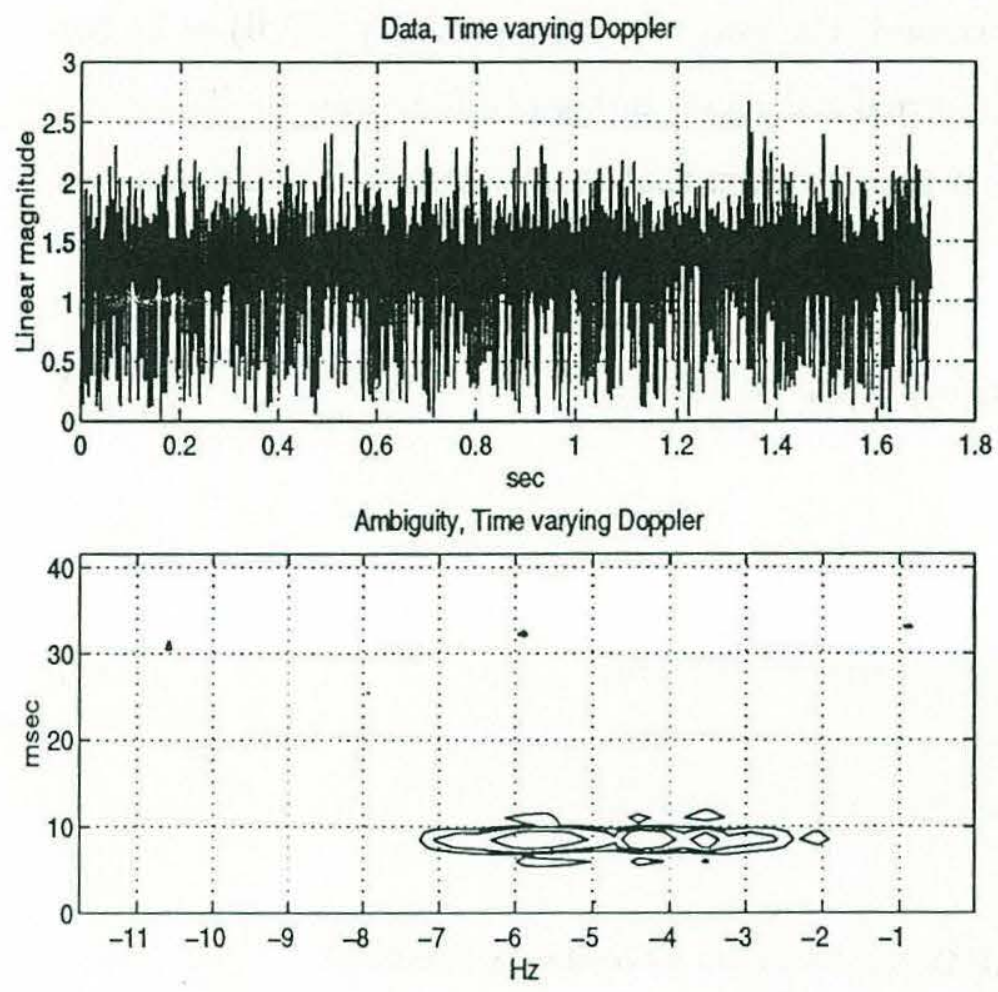

Figure 2-3: The complex envelope (upper panel) and $3 \mathrm{~dB}$ contours of the crossambiguity function (lower panel) for a signal with time-variant Doppler.

surface having a long swell, and the swell period is shorter than 2 sec. The upper panel of Fig. 2-3 shows the absolute value in linear scale of the complex envelope at 2 samples per symbol. The lower panel shows the cross-ambiguity function contours for this signal, and it has a mean Doppler of $-4 \mathrm{~Hz}$ but as the Doppler varies there are components on $-5.5 \mathrm{~Hz}$ and $-4.5 \mathrm{~Hz}$. The exact shape of the cross-ambiguity function in the case of a time-variant Doppler will depend on the time variation.

In order to compute the cross-ambiguity function as shown in Fig. 2-4 in a realistic receiver synchronization is necessary. Our current interest is merely to demon- 
strate different signals generating similar cross-ambiguity functions. Therefore we defer the synchronization discussion to Section 4.1 .5 where we explain how this is carried out. In Fig. 2-4 is shown a signal arriving over two ray paths with different
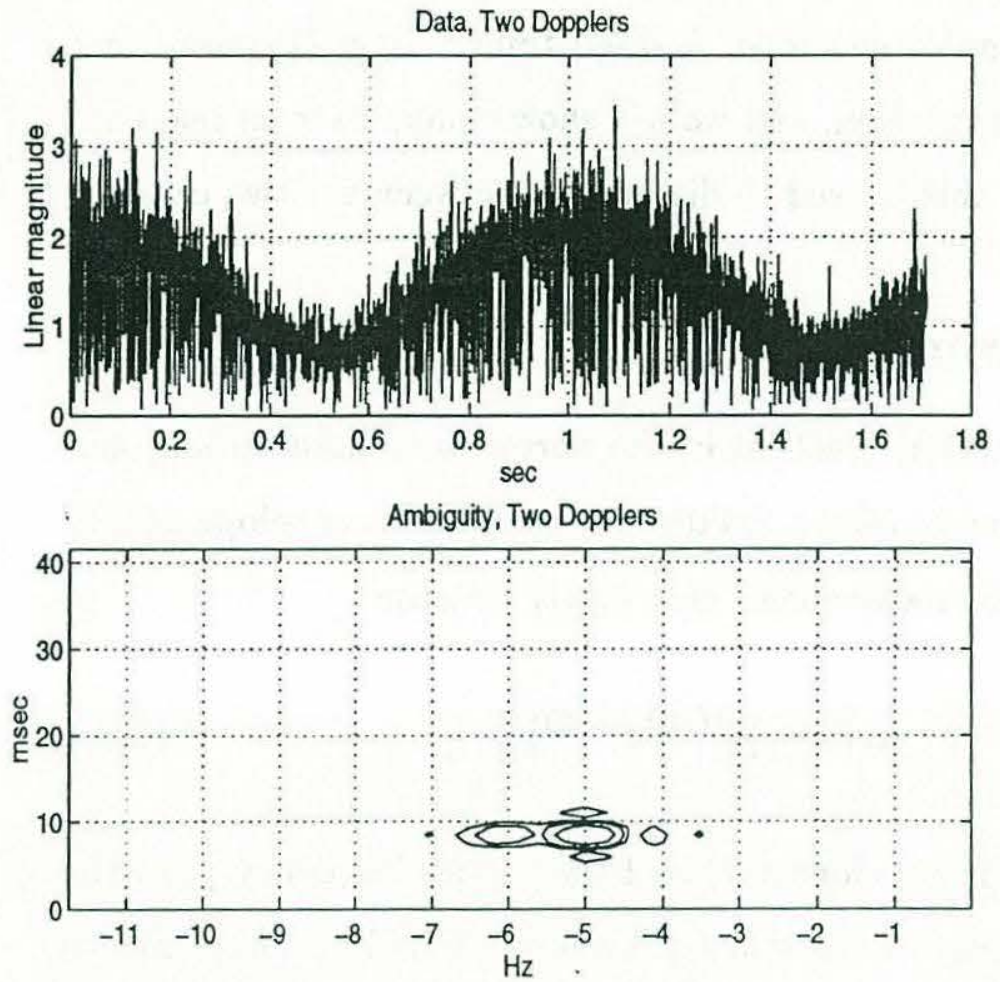

Figure 2-4: The complex envelope (upper panel) and $3 \mathrm{~dB}$ contours of the crossambiguity function (lower panel) for a signal with two Dopplers.

Doppler shifts. This would take place if one ray is a direct path and the other is interacting with the moving ocean-surface and the transmitter is close to the surface so that the ray travel times are almost equal. The contour plot of the cross-ambiguity function in the lower panel shows the two Doppler components of this signal, and their relative strength is a function of the relative strength of the signal on the two ray paths. The composite signal represented by its complex envelope is shown in the upper panel, and as the two ray paths interfere constructively or destructively the envelope of the signal varies. We have coherent channel fades when the two ray paths interfere destructively, and the depth of the fade is given by the relative strength of 
the two rays whereas the duration is given by the difference in Doppler. A coherent channel fade can only be compensated by use of redundancy in the symbol sequence in the way it is implemented with coding.

Even though the cross-ambiguity function for the two figures are similar we can see a major difference in the complex envelope: A single time-variant Doppler is not accompanied by an envelope modulation, and we will show examples from real data in the following chapters where this is used to distinguish between the two cases.

\subsubsection{Narrowband assumption}

We now consider the approximation involved in the narrowband assumption, and first we comment on the complex envelope notation. The complex envelope $x(t)$ of a signal $x_{0}(t)$ is convenient in the narrowband case partly because

$$
x_{0}(t)=\operatorname{Re}\left[|x(t)| e^{j L x(t)} e^{j 2 \pi f_{c} t}\right]
$$

allows us to identify the complex envelope $x(t)$ and the carrier frequency $f_{c}$ of the signal. If $x_{0}(t)$ is a wide band signal we can still express it by (2.37) but the quantities $x(t)$ and $f_{c}$ loose their meaning as the complex envelope and the carrier frequency. For ease of notation we retain this representation, and we take $f_{c}$ to be the center frequency of the frequency band of $x_{0}(t)$.

The effect of Doppler is more profound than a shift in frequency. In its fundamental form it involves the transformation from a stationary to a moving coordinate system resulting in time dilation. The distance between the coordinate systems is the range, and the motion is described by the time derivatives of the range. The relative motion is in general arbitrarily complex with non-zero relative speed, acceleration, change in acceleration, etc. We assume that effects from acceleration and higher order derivatives of the range are small and negligible. The first order effect,

not accounted for in the narrowband assumption, is non-zero speed. Specifically, if 
we have a sound pulse $x(t)$ traveling with speed $c$ in one coordinate system we have $x\left(\left(1+v_{s} / c\right) t\right)$ in a coordinate system that is moving with speed $v_{s}$ relative to the first one, and we can find the Doppler shift $\nu$ by

$$
\frac{v_{s}}{c}=\frac{\nu}{f_{c}}
$$

In addition to the time dilation it can be shown that the Doppler effect also results in amplitude distortion of the reflected waveform for a moving scatterer, and this is reported both for electro magnetic [83] and acoustic [62] signals. Therefore a generalized cross-ambiguity function is given by [83]

$$
\begin{aligned}
\Theta\left(\tau, \nu_{0}, \nu_{1}\right)= & \mid b\left(\nu_{0}\right) b\left(\nu_{1}\right) \int_{T} x\left(\left(1+\nu_{0} / f_{c}\right)(t+\tau / 2)\right) y^{*}\left(\left(1+\nu_{1} / f_{c}\right)(t-\tau / 2)\right) \times \\
& \left.e^{j 2 \pi\left(\nu_{0}-\nu_{1}\right) t} d t\right|^{2}
\end{aligned}
$$

where $b\left(\nu_{0}\right), b\left(\nu_{1}\right)$ accounts for the amplitude distortions at Doppler $\nu_{0}, \nu_{1}$. The amplitudes affect the shape of the ambiguity function, but the effect is small [62] for reasonable Doppler shifts, therefore we neglect this effect. The generalized crossambiguity function (2.39) is identical with the narrowband cross-ambiguity function (2.32) if we neglect the amplitude distortions, use a matched filter at $\nu_{0}=0$ and neglect the time dilation in the received signal $y(t)$. We now turn to the time dilation, and we remember that the ambiguity function can be interpreted as a matched filter. Thus the effect of neglecting the time dilation in the envelope of $x_{0}(t)$ is to use a slightly mismatched filter which will introduce a coherence loss. A common rule for when we can neglect the time dilation, based on limiting this coherence loss [83], is given by

$$
T W<0.1 \frac{c}{v_{s}}
$$

where $W$ is the bandwidth, $T$ is the signal duration and $v_{s} / c$ is known as the acoustic 
Mach number. This may or may not be violated in underwater communication channels, and we illustrate this with two examples.

Example 1 A receiver using RLS in decoding mode has a forgetting factor of $\lambda=.99$. Thus the number of samples used is approximately $1 /(1-\lambda)$ and since the symbol duration is $1 / W$ the averaging window is $T=1 /[(1-\lambda) W]$. Thus $T W=100$. For $c=1500 \mathrm{~m} / \mathrm{s}$ and $v_{s}=1 \mathrm{~m} / \mathrm{s}$, corresponding to $\nu=13 \mathrm{~Hz}$ for $f_{c}=20 \mathrm{kHz}$, the narrowband assumption (2.40) holds.

Example 2 We compute the cross-ambiguity function as given by (2.32) using 1 sec of data with bandwidth $600 \mathrm{~Hz}$. For $c=1500 \mathrm{~m} / \mathrm{s}$ and $v_{s}=1 \mathrm{~m} / \mathrm{s}(2.40)$ is violated.

We note that the effect of violating (2.40) in Example 2 is a gradual coherence loss. It is not catastrophic, and it can be compensated.

For a simple Doppler shift the time dilation is compensated by applying the inverse transformation of the one implied by the Doppler shift, and this can be carried out in the receiver if an estimate of the Doppler shift is available.

\subsection{Doppler lines and delay lines}

The doubly spread underwater communication channel exhibits both time and frequency dispersive fading that is caused by the Doppler and delay spread of the medium and by the transmit/receive platforms. Both delay spread and Doppler spread are forms of dispersion, and there is a close connection between channels exhibiting delay spread and channels exhibiting Doppler spread: If we think of the time domain and frequency domain as dual domains the delay and Doppler spread channels may be thought of as duals. This concept of duality is treated in depth in [8], and the reader should see this reference for definitions and implications of duality. We present here the concepts that are used in Chapter 4, and one of them is 
the "Doppler line" filter. The notion of a filter is often used for a device that makes a weighted sum of differently delayed versions of a signal. We use the name "delay line" for this device, and "Doppler line" for the device that makes a weighted sum of differently Doppler shifted versions of the signal. They are both filters but in dual domains.

The receivers derived and analyzed in Chapter 4 all work on discrete time signals, and the results from this section are used in the analysis and derivation of those receivers. Also the duality between delay lines and Doppler lines is developed by using the discrete time devices FIR and IIR delay lines, and for these reasons we now change to discrete time. The results from Section 2.3 all have straightforward discrete time counterparts, and they will be invoked as needed.

\section{Discrete time and frequency representation}

The delay-Doppler-spread function presented in (2.2) is discussed in [7], and this reference also contains a discussion of sampled channel models. We use in Chapter 4 a channel representation that is discrete in both delay and Doppler, and the assumptions in going both from continuous to discrete time and frequency is presented by using some results from [7]. The delay-Doppler-spread function is given by (2.2) and repeated here for convenience:

$$
y(t)=\iint x(t-\xi) U(\xi, \nu) e^{j 2 \pi \nu t} d \xi d \nu .
$$

Our goal is a discrete representation of (2.41). We define the time-variant transfer function as

$$
T(f, t)=\iint U(\xi, \nu) e^{j 2 \pi(\nu t-\xi f)} d \xi d \nu
$$


where $T(f, t)$ is related to the input delay-spread function by

$$
h(t, \tau)=\int T(f, t) e^{j 2 \pi f \tau} d f .
$$

By inserting the inverse Fourier-transform of (2.42) in (2.41) we get

$$
y(t)=\int X(f) T(f, t) e^{j 2 \pi f t} d f
$$

The reason for the name of $T(f, t)$ is seen from (2.44) since this is the generalization of the time-invariant transfer function. There are various constraints that are naturally associated with a realistic communication system, and by imposing different constraints one can arrive at a discrete representation of (2.41) via different routes. We now assume that there is an input frequency and output time constraint, so that

$$
\begin{aligned}
X(f) & =0,\left|f-f_{i}\right|>W_{i} / 2 \\
y(t) & =0,\left|t-t_{o}\right|>T_{o} / 2 .
\end{aligned}
$$

The input signal, here represented by its complex envelope, is assumed to be band limited to the band $W_{i}$ centered at $f_{i}$ and the complex envelope of the output signal $y(t)$ is assumed to be time limited to the time $T_{o}$ centered at $t_{o}$, therefore it can not be band limited. In order to obtain (2.46), and also to discretize $y(t)$ in (2.53), we assume that $y(t)$ has most of its energy in a band $W_{o}$. Thus $y(t)$ is approximately both time limited and band limited, and it is clear from the first part of (2.45) that (2.44) can be expressed as

$$
y(t)=\int X(f) \operatorname{rect}\left(\frac{f-f_{i}}{W_{i}}\right) T(f, t) e^{j 2 \pi f t} d f
$$


and also from the second part of (2.45) we write (2.46) as

$$
\begin{aligned}
y(t)=y(t) \operatorname{rect}\left(\frac{t-t_{o}}{T_{o}}\right)= & \int X(f) \operatorname{rect}\left(\frac{f-f_{i}}{W_{i}}\right) T(f, t) \operatorname{rect}\left(\frac{t-t_{o}}{T_{o}}\right) \times \\
& e^{j 2 \pi f t} d f .
\end{aligned}
$$

We assume in (2.46) that $y(t)$ is approximately band limited so that it has most of its energy in a band $W_{o}$. Then we may consider it irrelevant what value $T(f, t)$ has outside the intervals given in (2.45). Therefore (2.47) yields

$$
\tilde{y}(t)=\int X(f) \tilde{T}(f, t) e^{j 2 \pi f t} d f
$$

where we have defined

$$
\tilde{T}(f, t)=\sum_{k=-\infty}^{\infty} \sum_{n=-\infty}^{\infty} T\left(f-k W_{i}, t-n T_{o}\right)
$$

and $\tilde{y}(t)$ is the periodic extension of $y(t)$, i.e., $\tilde{y}(t)=y(t)$ for $\left|t-t_{0}\right|<T / 2$. Since we are considering this time interval only we substitute $y(t)$ for $\tilde{y}(t)$ in the following. The Fourier-transform of (2.49) is

$$
\tilde{U}(\xi, \nu)=\frac{1}{W_{i} T_{o}} \sum_{l=-\infty}^{\infty} \sum_{k=-\infty}^{\infty} U\left(\frac{l}{W_{i}}, \frac{k}{T_{o}}\right) \delta\left(\xi-\frac{l}{W_{i}}\right) \delta\left(\nu-\frac{k}{T_{o}}\right)
$$

and $\tilde{U}(\xi, \nu)$ satisfies (2.41) in $\left|t-t_{0}\right|<T / 2$ because $\tilde{T}(f, t)$ satisfies (2.48). Therefore inserting $(2.50)$ in $(2.41)$ yields

$$
y(t)=\sum_{l=-\infty}^{\infty} \sum_{k=-\infty}^{\infty} U(l, k) x\left(t-\frac{l}{W_{i}}\right) e^{j 2 \pi\left(k / T_{0}\right) t}
$$

where we have defined

$$
\frac{1}{T_{0} W_{i}} U\left(\frac{l}{W_{i}}, \frac{k}{T_{0}}\right)=U(l, k) .
$$


Because $y(t)$ is approximately time limited and band limited the sampling theorem approximately yields

$$
y(t) \approx \sum_{m=-\infty}^{\infty} y\left(\frac{m}{W_{o}}\right) \operatorname{sinc}\left[W_{o}\left(t-\frac{m}{W_{o}}\right)\right]
$$

where we define

$$
\operatorname{sinc}(x)=\frac{\sin (\pi x)}{\pi x}
$$

By using (2.53) in (2.51) we get

$$
y(t)=\sum_{m, l, k} U(l, k) x\left(\frac{m}{W_{o}}-\frac{l}{W_{i}}\right) \operatorname{sinc}\left[W_{o}\left(t-\frac{m}{W_{o}}\right)\right] e^{j 2 \pi k m /\left(T_{o} W_{o}\right)} .
$$

In a typical communication system we have that the Doppler spread is much less than $W_{i}$, and in practice we have $W_{i} \approx W_{o}$. By using this in (2.55) and assuming that we want to obtain $y\left(n / W_{o}\right)$ we get

$$
y(n)=\cdot \sum_{l, k} U(l, k) x(n-l) e^{j 2 \pi k \Delta \nu n}
$$

where we have defined $\Delta \nu=1 /\left(T_{o} W_{o}\right)$ and changed notation so that $y\left(n / W_{o}\right) \Rightarrow$ $y(n)$ and $x\left(n / W_{o}\right) \Rightarrow x(n)$.

We now look at some of the features of Doppler lines, and connect them to their duals the more frequently encountered delay lines. Let us assume a purely Doppler spread channel model. In continuous time we have

$$
y(t)=x(t) \int F(\nu) e^{j 2 \pi \nu t} d \nu+w(t) .
$$

From (2.41) we see that this is a special case where

$$
U(\xi, \nu)=F(\nu) \delta(\xi)
$$


therefore from (2.56) we have

$$
y(n)=x(n) \sum_{k=-\infty}^{\infty} F(k) e^{j 2 \pi k \Delta \nu n}+w(n) .
$$

If we assume that $F(k) \approx 0$ for $k$ outside $0 \leq k<M(2.59)$ yields

$$
y(n)=x(n) \sum_{k=0}^{M-1} F(k) e^{j 2 \pi k \Delta \nu n}+w(n) .
$$

Note that the model (2.60) implies a time-variant channel, it is therefore expected that the device needed to compensate this channel is time-variant. Thus the Doppler line is a time-variant gain unlike its dual the delay line.

\section{Finite frequency spread (FFS)}

Consider the Doppler line in Fig. 2-5. The structure is identical to that of a FIR

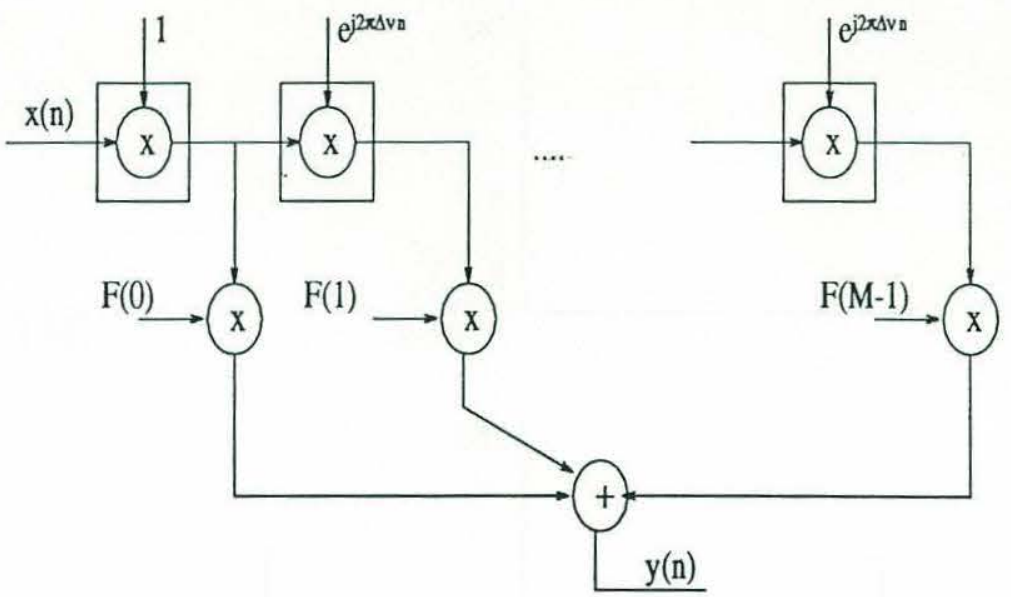

Figure 2-5: The FFS Doppler line.

delay line the only difference being that the Doppler shift is used in place of the delay. The boxes with the multiplicators in Fig. 2-5 are mixers both supplying the next mixer and the local weight with its output. The FFS is the dual of the FIR because as an FIR delay line has finite impulse response, as will be shown below, its 
dual the FFS has finite frequency spread. The picture in Fig. 2-5 is written as

$$
y(n)=x(n) \sum_{k=0}^{M-1} F(k) e^{j 2 \pi k \Delta \nu n}
$$

and by taking the discrete time discrete frequency Fourier-transform, assuming $k \Delta \nu=k / N$ with $N$ being window length, we get

$$
Y(k)=\sum_{i=0}^{M-1} F(i) X(k-i)
$$

where $X(k)$ and $Y(k)$ have period $N$. The nonzero support of $F(i)$ is

$$
0 \leq i \leq M-1 \text {. }
$$

If $x(n)$ is a single frequency so that $X(k)=\delta\left(k-k_{0}\right)$ for $0 \leq k<N$ we have a situation as shown in Fig. 2-6. We find from (2.62) that
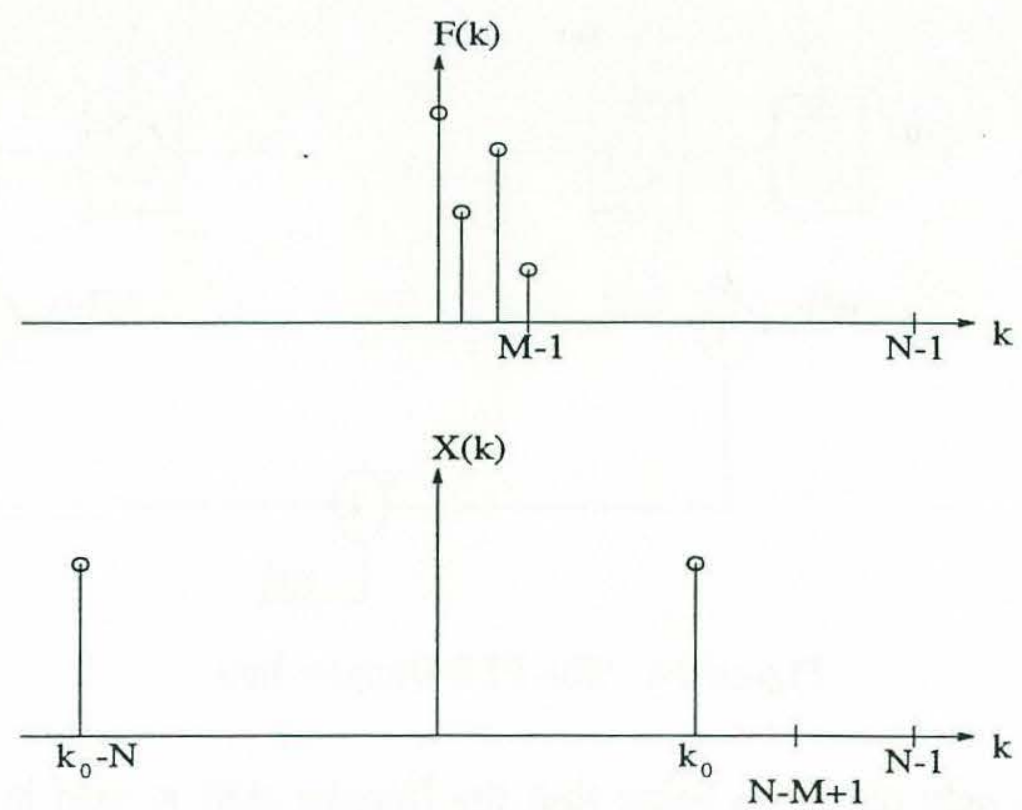

Figure 2-6: The convolution of the two signals yields the output of the FFS Doppler line. 


$$
Y(k)=F\left(k-k_{0}\right),
$$

and from Fig. 2-6 we see that no aliasing takes place if

$$
k_{0}<N-M+1 \text {. }
$$

Thus the single frequency $k_{0}$ has been spread on the finite interval of width $M$ and thereby this Doppler line gets the name "FFS". The aliasing requirement constrains the bandwidth of $x(n)$ but for practical communication channels and signals $M \ll N$ so that this constraint is not severe.

\section{Infinite frequency spread (IFS)}

The picture in Fig. 2-5 defines the relationship between $x(n)$ and $y(n)$ as given in (2.61), and conversely (2.61) can be depicted as shown in Fig. 2-5. The duality between the FFS Doppler line and the FIR delay line is shown in the previous section, and we now look for the dual of an IIR delay line. It is clear that this device must obey

$$
Y(k)=X(k)-\sum_{i=1}^{M-1} F(i) Y(k-i)
$$

which is the dual of the equation for an IIR delay line. We note that this equation always gives aliasing but that in the communication systems of concern the condition $M \ll N$ may yield small aliasing. A necessary condition for the aliasing to be small is that the IFS is stable which means that all $M$ roots $r_{l}$ of

$$
r^{k}+\sum_{i=1}^{M-1} F(i) r^{k-i}=0
$$




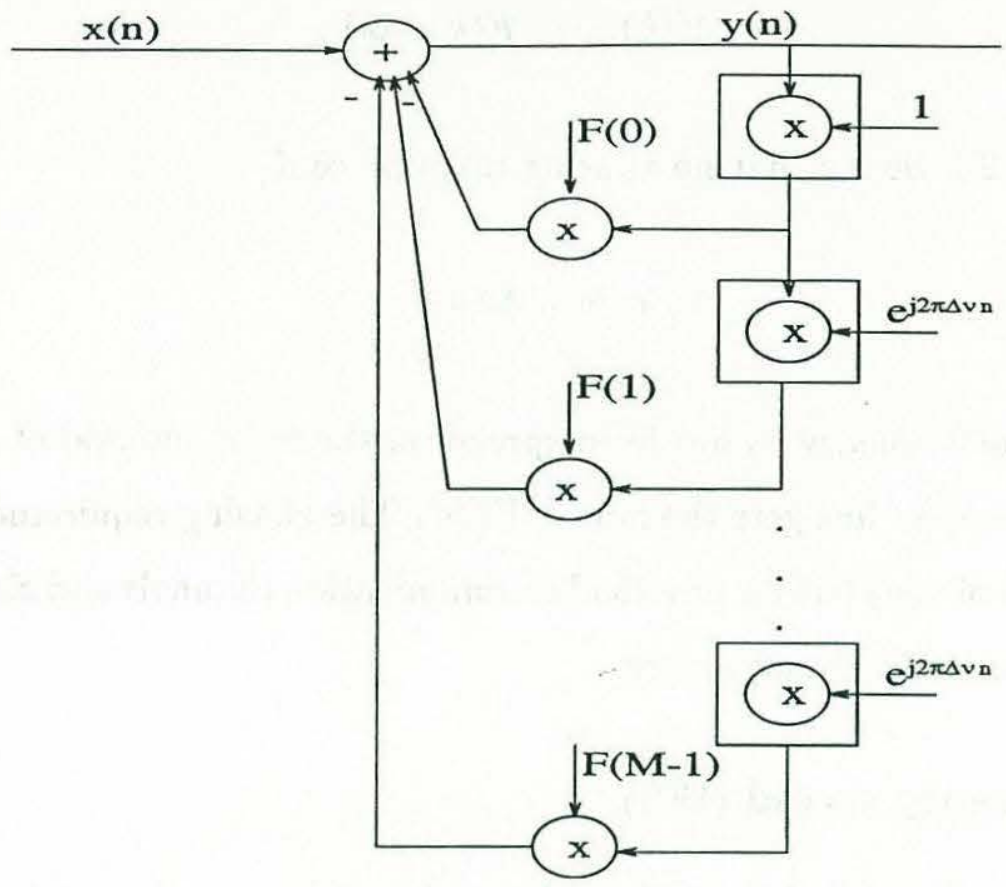

Figure 2-7: The IFS Doppler line.

have magnitude less than unity. By taking the inverse Fourier-transform of (2.66) we get

$$
y(n) \sum_{k=0}^{M-1} F(k) e^{j 2 \pi k \Delta \nu n}=x(n) .
$$

where we have defined $F(0)=1$, and this equation suggests the picture in Fig. 2-7. Since there is no time delay the interpretation of this figure is ambiguous and it must be interpreted by means of (2.68). By defining

$$
f(n)=\sum_{k=0}^{M-1} F(k) e^{j 2 \pi k \Delta \nu n}
$$

and using (2.68) we have

$$
y(n)=\frac{x(n)}{f(n)} .
$$


Assume that the communication channel is modeled as an FFS Doppler line, i.e., the received signal is composed of rays with different Doppler shifts and almost equal travel times. In this case the receiver that compensates this is an IFS Doppler line and this is used in Chapter 4.

\section{Eigenfunctions}

The eigenfunctions for delay lines are complex exponentials. When $e^{j 2 \pi k \Delta \nu n}$ is the input to a delay line with frequency response $H(k)$ the steady state output is

$$
|H(k)| e^{j L H(k)+j 2 \pi k \Delta \nu n} .
$$

The output is a scaled version of the input which is the identifying feature of an eigenfunction. The eigenfunctions for Doppler lines are delta functions in time or complex exponentials in frequency which can be seen by insertion. In the case of an FFS we have that for an input

$$
\begin{aligned}
x(n) & =\delta\left(n-n_{0}\right) \\
X(k) & =e^{j 2 \pi k \Delta \nu n_{0}}
\end{aligned}
$$

using (2.61), the output is

$$
y(n)=\sum_{k=0}^{M-1} F(k) e^{j 2 \pi k \Delta \nu n_{0}} \delta\left(n-n_{0}\right)
$$

which is a scaled version of the input. The scaling factor may be identified as the inverse Fourier-transform of $F(k)$ :

$$
f\left(n_{0}\right)=\sum_{k=0}^{M-1} F(k) e^{j 2 \pi k \Delta \nu n_{0}}=\left|f\left(n_{0}\right)\right| e^{j L f\left(n_{0}\right)} .
$$


The quantity $\angle H(k)$ in (2.71) is the phase delay of a delay line, and it tells how much the phase of the output lags the phase of the input sinusoid for a given frequency $k$. The dual quantity $\angle f\left(n_{0}\right)$ may be interpreted as the delay phase, because it tells what the phase difference between input and output is for a given delay. Some FFS's have linear delay phase, as some of their dual counterparts the FIR's have linear phase delay. Correspondingly no IIR's have linear phase delay, and no IFS's have linear delay phase.

We can make some physical interpretation if we relate these facts to the underwater communication channel. If we use a CW signal at the fixed transmitter, and measure the phase angle with respect to the transmitted phase at the fixed receiver this angle is constant and equal to $\angle H(k)$ if the medium between the transmitter and receiver contains only fixed point reflectors of constant strength. If the point reflectors are moving, so that the medium is time-variant, the phase from the $\mathrm{CW}$ as it is scattered off a particular point reflector will change when it is measured at the receiver. The reason for this is that the total path length between the transmitter, point reflector and the receiver is changing (the point reflector is moving), therefore the phase also changes with time. This change is given by the delay phase $\angle f\left(n_{0}\right)$.

Summary This concludes the presentation of the tools useful for characterization of the underwater communication channel, and they are used in Chapter 4. The LTV system is characterized by means of the input delay-spread function $h(t, \tau)$ which has the physical interpretation of non-moving scintillating scatterers at different delays, and the delay-Doppler-spread function $U(\tau, \nu)$ which has the physical interpretation of non-scintillating scatterers moving at different speeds and delays. The signal from each scatterer is interpreted as a WSS process and two scatterers produce uncorrelated scattering. This is the WSSUS assumption, and the importance of it is among other things that a channel scattering function $S(\xi, \nu)$ can be defined. The physical interpretation of this quantity is as a double power density spectrum in delay 
and Doppler, so that it gives the distribution of energy vs these two variables. We present the ambiguity function and generalize this to the cross-ambiguity function which can be used to estimate the channel scattering function from the knowledge of input and output channel data only. We also point out that there may be different physical processes that give similar shapes for the cross-ambiguity function, and this is illustrated in the case of Doppler spread on synthetic data where we observe that composite information from both the cross-ambiguity function and the complex envelope of the signal enables us to distinguish between these two cases. We also develop the concept of duality and discuss the time-variant devices FFS and IFS Doppler lines, and these are used in the derivation of a receiver for doubly spread channels in Section 4.3.4. 


\section{Chapter 3}

\section{Simulations and measurements of communication channels}

\subsection{Simulation tool}

The purpose of any simulator is to realistically model some phenomenon under controlled conditions, and here we want to gain some insight into the important features when using the ocean to transmit information by means of acoustical waves. The frequencies in question may range from $5 \mathrm{kHz}$ to $50 \mathrm{kHz}$, and this suggests that within limits a ray model for the acoustic field is appropriate. This is also the basis for the time-variant FIR model to be described below. The overall simulator setup is shown in Fig. 3-1.

\subsubsection{Time-variant FIR model}

The time-variant FIR model for the channel is expressed in terms of the time-variant attenuation coefficients from the ray model of the acoustic field. The total acoustic field at any reception point is decomposed into rays, so that the complex acoustic pressure from each ray is derived from each attenuation coefficient $h_{i}(t)$. The input 


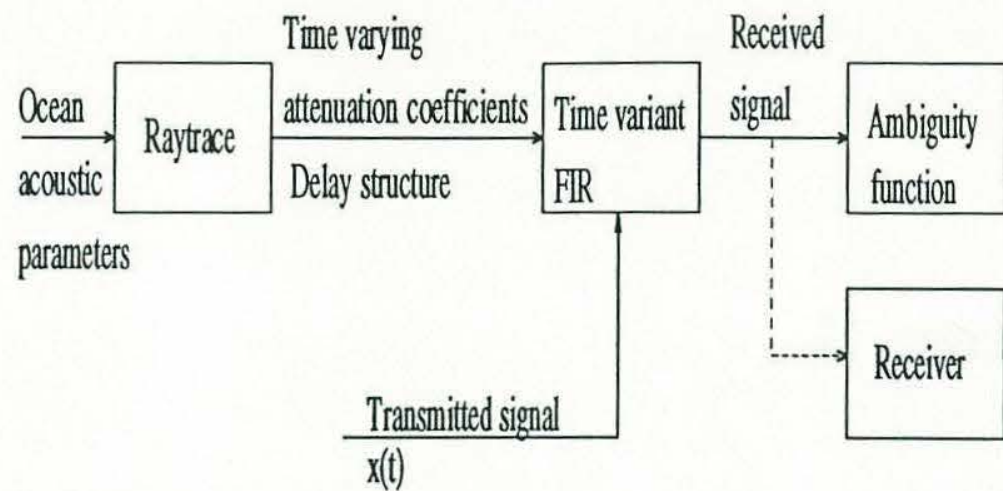

Figure 3-1: The simulator is based on ocean related parameters feeding a raytrace model.

to the system is the transmitted signal $x(t)$, and into the time-variant FIR filter goes $\mathbf{x}(t)$ which is given by

$$
\mathbf{x}(t)=\left[x\left(t-t_{0}\right) \cdots x\left(t-t_{L-1}\right)\right]^{T}
$$

if the system order is $L . L$ is also the number of eigenrays of the system, and $t_{L-1}$ is the maximum delay of the system. The complex attenuation coefficients $h_{i}(t)$ are contained in the filter tap vector given by

$$
\mathbf{h}(t)=\left[h_{0}(t) \cdots h_{L-1}(t)\right]^{T} .
$$

Each ray has a complex attenuation coefficient, and $h_{i}(t)$ is the coefficient for ray $i$ arriving at time $t$. The received signal is given by

$$
\begin{aligned}
y(t) & =\mathbf{h}^{T} \mathbf{x}+w(t) \\
& =\sum_{i=0}^{L-1} h_{i}(t) x_{i}+w(t)
\end{aligned}
$$

where $x_{i}$ is element number $i$ of $\mathrm{x}(t)$ and $w(t)$ is Gaussian complex white noise. Note that rays arriving arbitrarily close in time are allowed in this model. For example if 
ray $i$ and ray $j$ have the same travel time this is incorporated by letting $t_{i}=t_{j}$ in (3.1). The analogy between the ray model acoustic field and the linear time-variant system is shown in Fig. 3-2, where the signal arrives over four different paths and
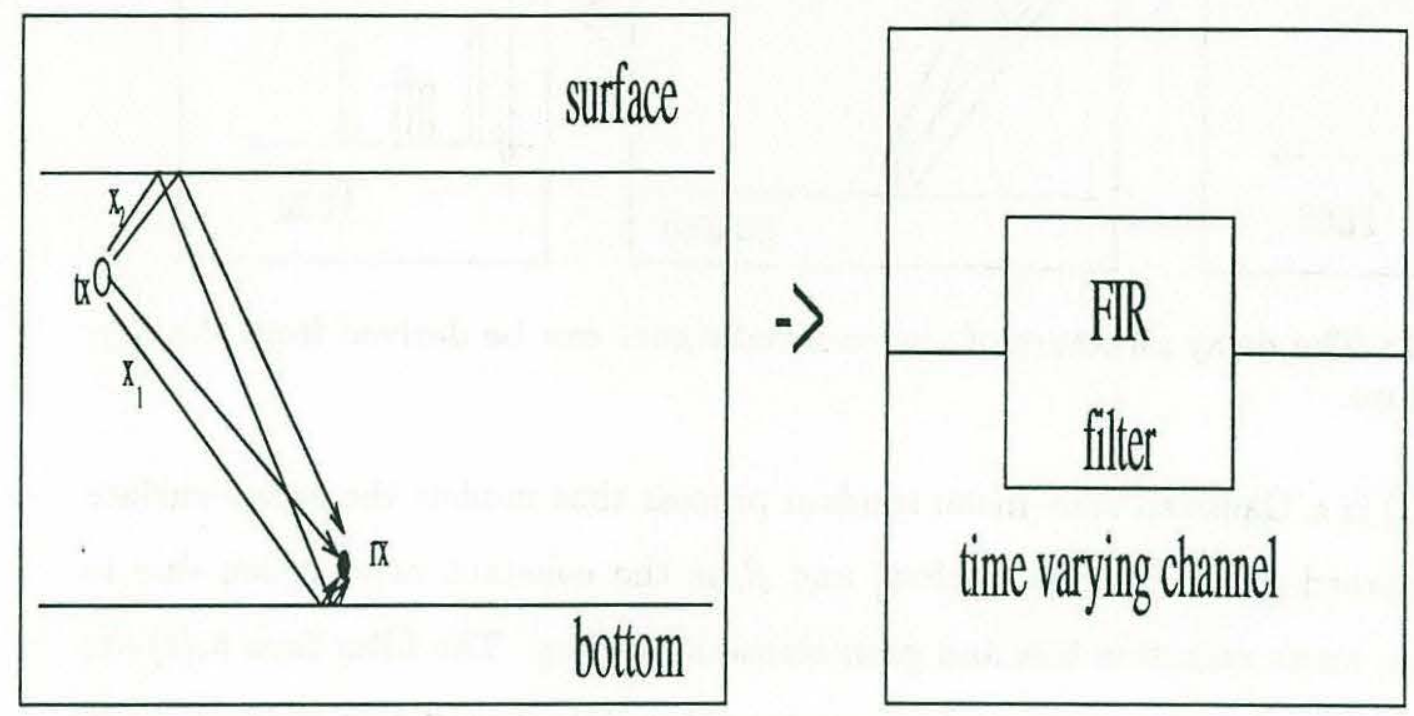

Figure 3-2: Time varying, clustered channel with four taps in the FIR filter.

each of these paths has different attenuation and arrival time so that the FIR filter in this case has four taps. The number of significant rays depends on the channel geometry, sound speed profile and boundary interaction. It varies significantly as a function of these parameters. The analogy depicted in Fig. 3-2 shows an example of how the FIR parameters and delays are given by the ray model, and Fig. 3-3 shows conceptually how the received signal is derived from the ray model. If the transmitted signal is a short pulse, indicated in Fig. 3-3, the ray model predicts that this pulse travels over the different ray paths, and arrives at the receiver as four delayed and attenuated replicas of the transmitted signal. The delays are given by computing the travel time for each ray, and the complex attenuation coefficients are modeled as

$$
h_{i}(t)=\beta_{i}\left[1+s_{i}(t)\right] e^{j 2 \pi \nu_{i} t}
$$




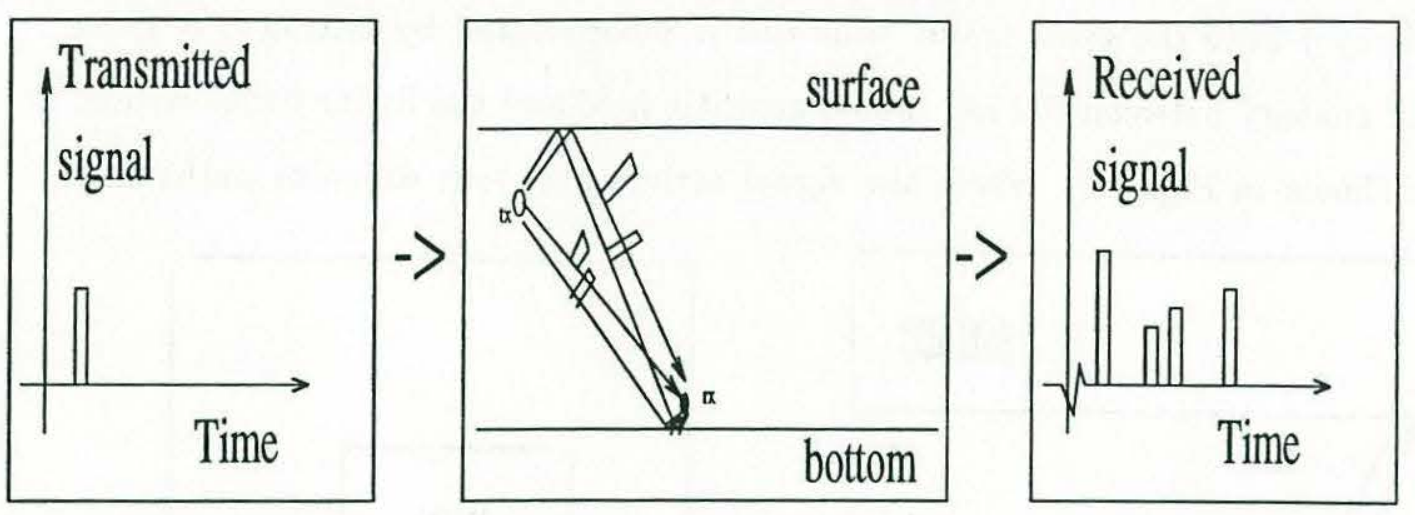

Figure 3-3: The delay structure of the received signal can be derived from the raytrace picture.

where $s_{i}(t)$ is a Gaussian zero-mean random process that models the ocean-surface Doppler spread to be described below, and $\beta_{i}$ is the constant attenuation due to absorption, mean reflection loss and geometrical spreading. The filter taps $h_{i}(t)$ are time varying random variables, and $\nu_{i}$ can be either deterministic or random. If $\nu_{i}$ is deterministic its value gives a Doppler shift modeling relative motion between the scattering boundaries, transmitter and receiver. We use the model with deterministic $\nu_{i}$ in which case we have

$$
E\left[h_{i}\right]=\beta_{i} e^{j 2 \pi \nu_{i} t}, \quad \operatorname{var}\left[h_{i}\right]=\left|\beta_{i}\right|^{2} \operatorname{var}\left(s_{i}\right) .
$$

The time correlation for each tap is

$$
R_{h h}(\tau)=\left|\beta_{i}\right|^{2} e^{-j 2 \pi \nu_{i} \tau}\left(1+R_{s s}(\tau)\right)
$$

where $R_{s s}(\tau)$ is the autocorrelation of $s_{i}(t)$.

\section{Modeling of surface Doppler spread}

When rays are reflected from the ocean-surface their frequency spectrum is broadened because the scatterers are moving at different speeds. The frequency spreading 
of a ray due to ocean-surface motion can be estimated by characterizing the oceansurface with its spatial wavenumber power density spectrum, and the dispersion relation for gravity waves can be used to obtain the frequency temporal spectrum. Thus, by assuming a known ocean-surface spectrum (e.g., Pierson-Moskowitz), the Doppler spread can be estimated [14]. We now consider a single ray that is scattered from a rough surface, and we temporarily drop the subscript $i$. If a single ray is scattered from the rough surface, which we want to characterize with $s(t)$, the direction of the reflected ray varies over a wide range of angles as the surface changes over time. Fig. 3-4 shows a snapshot of the time varying rough surface at time $t_{0}$. The process that we want to characterize is given by $s\left(t, r_{0}\right)=s(t)$ at some specific location $r_{0}$ on the surface. It can be shown [14] that the time-averaged reflection

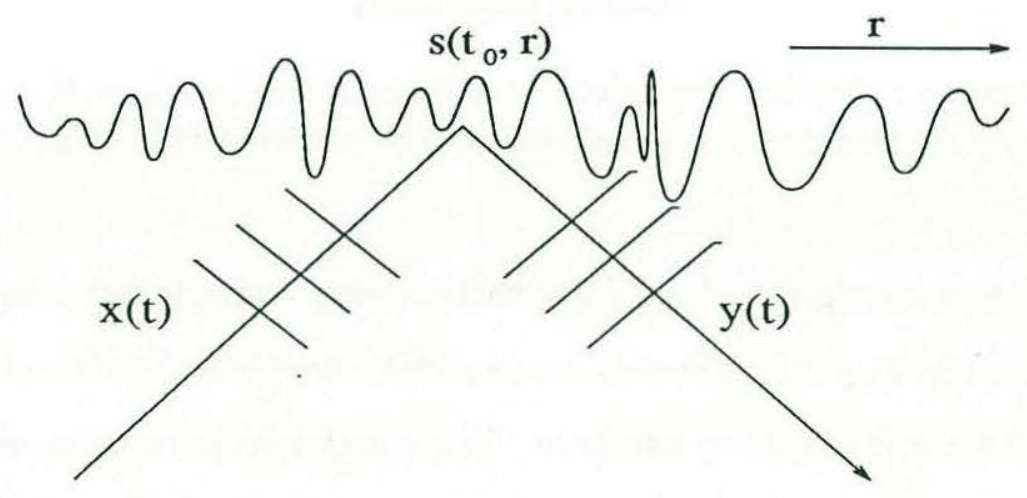

Figure 3-4: Surface Doppler spread, a snapshot of the time-variant rough surface which is modeled as a random process. We use the time-averaged reflection coefficient, therefore only the specular direction is shown

coefficient falls off rapidly for angles away from the specular direction with respect to the mean surface (which is horizontal). This leads to the simplification that only the ray in the specular direction is considered when we are trying to obtain the response from the rough surface, and it is sometimes called the Eckart formulation for rough surface scattering [14].

Fig. 3-5 shows the time-averaged reflection coefficient vs grazing angle [22] which is the angle between the horizontal plane and the ray direction. The reflection 


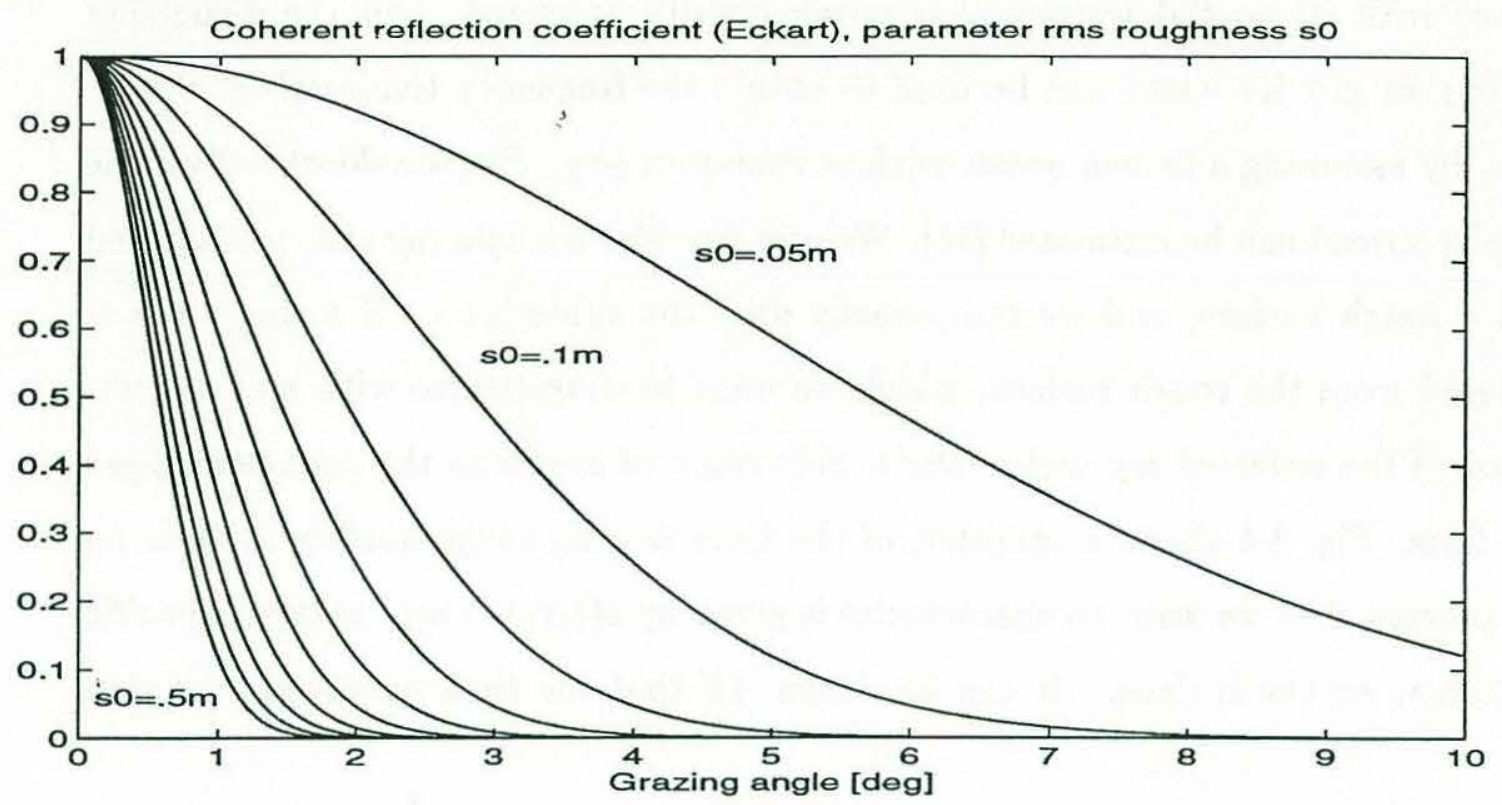

Figure 3-5: Average reflection coefficient vs grazing angle (angle with respect to the horizontal plane). The curves are parameterized by the rms roughness $s 0$ going from $0.05-0.5 \mathrm{~m}$.

coefficient is also a strong function of the root-mean-square (rms) roughness of the surface, as shown in Fig. 3-5. There is a connection between the Eckart formulation and the model for $h_{i}(t)$ that we use here. The roughness parameter $s 0$ in Fig. 3-5 is the temporal variance $R_{s s}(0)$ of $s(t)$. The average reflection coefficient given in this figure is also related to $\beta$ in (3.4) which for each ray is made up of several components. The components are geometrical spreading with power loss roughly proportional to $r^{2}$ where $r$ is the range, attenuation measured in $\mathrm{dB} / \mathrm{km}$ which is proportional to $r$ with a frequency dependent constant of proportionality, and finally in the case of boundary interaction there is a grazing angle (angle with respect to the horizontal) dependent loss given by Fig. 3-5.

We now look for a way to characterize the surface Doppler spread when a complete statistical description of the Gaussian random process $s(t)$ is given by means of its power spectrum $S(\nu)$, see Fig. 3-6. To find a sample path $s(t)$ such that 


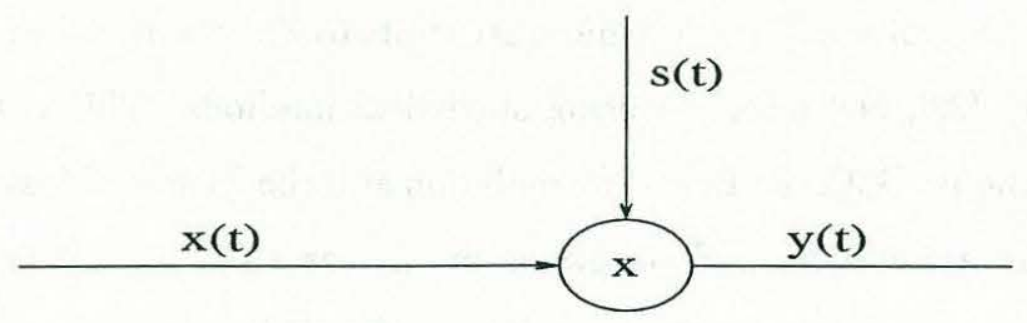

Figure 3-6: A simplified representation of the impact of a rough time varying surface on a ray.

$$
\mathcal{F}\left(R_{s s}(\tau)\right)=S(\nu)
$$

where $\mathcal{F}$ means Fourier transform we first discretize $S(\nu)$ which is the power spectral density of the random process $s(t) . S(k \Delta \nu) \Delta \nu$ is approximately the power in the frequency band of width $\Delta \nu$ centered at $k \Delta \nu$. Now we can write the sample path as

$$
s(t)=\sum_{k} \sqrt{S(k \Delta \nu) \Delta \nu} w(k) e^{-j 2 \pi k \Delta \nu t}
$$

where $w(k)$ is Gaussian, zero-mean, white noise. This is verified by computing the autocorrelation of $s(t)$ :

$$
\begin{aligned}
R_{s s}(\tau) & =E\left[s(t) s^{*}(t-\tau)\right] \\
& =\sum_{k} S(k \Delta \nu) \Delta \nu e^{-j 2 \pi k \Delta \nu \tau}
\end{aligned}
$$

which shows the desired relationship between $R_{s s}(\tau)$ and $S(\nu)$. We note that in order for $S(k \Delta \nu)$ to be an accurate representation of $S(\nu)$ the corresponding time function i.e., $R_{s s}(\tau)$, is time limited.

\section{Limitation of the statistical description}

The scattering from a rough, time-variant ocean-surface causing Doppler spread is usually not captured in a deterministic framework. The major reason for this is that the computations involved in obtaining the field scattered from a rough 
surface is rather involved. Therefore most attempts to characterize the ocean-surface scattering [14], [22], [99] resort to using statistical methods. This is the approach taken in this thesis. Both the Eckart formulation and the Pierson-Moskowitz surface wave spectrum are statistical descriptions of the sea surface. We use the Eckart formulation to argue that only the specular ray direction is significant for coherent signals, and the Pierson-Moskowitz spectrum to incorporate the frequency spread in the specular direction. These are both averages, and the Pierson-Moskowitz spectrum is obtained by observing sea surface wavenumber vectors over long time intervals which are averaged. In a similar manner the Eckart reflection coefficient [30] is obtained by repeatedly transmitting sound pulses and measuring the response of the scattered pulses. Fig. 3-5 is obtained in this way, and the original experiment has been repeated in slightly different ways. The results in a more controlled environment from an anechoic tank with a wind driven surface [23] are similar.

Thus the two statistical formulations used herein are only valid for the average result over many transmissions. In particular, in order to capture the statistical behavior, the duration of a data packet transmission should be long enough to capture the averaging effect. A typical data packet duration is $2 \mathrm{sec}$, and a surface swell may have a period of 5-10 sec. Thus the important feature of the surface in this scenario is the "snapshot" behavior rather than the statistical average. For example, for a long swell the most important feature is what the phase of the long wave is. This limits the accuracy of our models for ocean-surface Doppler spread, and this in turn impacts the data analysis. A more accurate way of calculating the effect of surface reflected rays is to use the simulator in the same manner as the Eckart reflection coefficient is obtained. I.e., to obtain the Doppler spread of a particular scenario we could run the simulator many times with a random surface at the top. Then the receptions should be averaged to capture the effect that is currently incorporated through the Eckart reflection coefficient.

This is not carried out in this thesis, and it is a potential improvement that 
would yield a more accurate Doppler spread. It would be an increasingly important modification as the packet length decreases, or as the low frequency content of the surface variation increases. The data analysis is also less accurate due to this fact. The simulator may be used to obtain statistics of the error by running it many times with different surface realizations.

\subsubsection{Raytrace}

The time-variant FIR model is in itself a sufficient model for the communication channel and it is used for this purpose [101], [82]. However, the input parameters are the time-variant filter tap values and locations and these must be specified when this approach is used. These parameters are not directly derived from quantities that can be measured in a real ocean communication channel, and the purpose of the raytrace is to supply the content and structure of the FIR filter tap vector $\mathbf{h}(t)$ in (3.3). The implementation of the time-variant FIR filter, given $\mathbf{h}(t)$, is as described in Section 3.1.1. This way of implementing the input to the time-variant FIR filter representing the communication channel assures that there is a physically sound coupling between the channel parameters and the real ocean. It enables the search for channels with pre-specified ambiguity function shapes such as large Doppler spreads.

\section{Input parameters}

The input parameters to the raytrace are

- Sound speed profile

- Vertical beamwidth for transmitter and receiver

- Carrier frequency

- Transmitter and receiver locations (range,depth) 
- Transmitter and receiver velocity

- Bottom and ocean-surface forward scatter values

- Ocean-surface Doppler spread

Sound speed profile The sound speed profile is constrained to be piecewise linear. There is no limit to how many line segments it contains, and thus arbitrary profiles can be modeled. The linearization modifies the raytrace, and it may introduce false regions of high intensity called false caustics. The effect is small and the deviation from the correct raytrace due to the linearization is negligible.

Vertical beamwidth for transmitter and receiver The vertical beamwidth of the transmitter yields the insonification area, and the beam can be tilted and set to arbitrary widths. The receiver vertical beam can also be of arbitrary width. The beamwidths determine how much boundary interaction there is, and this is an important factor in determining the delay spread.

Carrier frequency The carrier frequency is used to get Doppler shifts from transmitter and receiver velocities. It can also be used to get the Rayleigh parameter for the ocean-surface and bottom roughness.

Transmitter and receiver locations (range, depth) Transmitter and receiver locations obviously impacts both the delay and Doppler distributions.

Transmitter and receiver velocity The relative velocities give different Doppler shifts for each ray connecting the receiver and transmitter, therefore the result is a Doppler spread. The spread in this case consists of differential Doppler between the rays. 
Bottom and ocean-surface forward scatter values Bottom and ocean-surface forward scatter values are stored as look up tables, supplied via external files, and thus they can easily be modified. The Eckart formulation for rough surface scattering is used to fill the tables [14], [22], but this may be changed by supplying new tables. There is one scattering value for each grazing angle. When a ray is traced its grazing angle is known, and this is used to get an attenuation value from the table. The main task of the simulator is not to derive and implement new surface scattering theories, but rather to use the most appropriate of the existing ones to realize communication channels.

Ocean-surface Doppler spread The ocean-surface motion impacts the Doppler spread for surface reflected rays. A mean Doppler shift is in general present as a result of the fact that the water near the surface has a net velocity due to Stokes and Lagrangian drift [11]. This is not accounted for in the theory that we use, and thus it yields zero Doppler shift in the specular direction with respect to the mean surface. In the case of small Rayleigh parameter for the surface the method of small perturbation is used to estimate Doppler spread [14]. This method also yields zero Doppler spread in the specular direction. In the case of large Rayleigh parameter the Kirchoff approximation (tangent plane method) gives a Doppler spread $\Delta \nu$ in the specular direction [14]:

$$
\Delta \nu=c q \sin (\psi) v_{s}^{3 / 2}
$$

where $c=1.75 \times 10^{-2}$ is a constant, $q$ is the acoustic wavenumber, $\psi$ is the grazing angle and $v_{s}$ is the wind speed. With $20 \mathrm{kHz}$ carrier frequency $45 \mathrm{deg}$ grazing angle and $10 \mathrm{~m} / \mathrm{s}$ wind speed we get $12 \mathrm{~Hz}$ Doppler spread which is in good correspondence with some of the observed data. 


\section{Model limitations}

Ray modeling of the acoustic field is an approximation taken from geometrical optics. It is valid exactly as the carrier frequency goes to infinity but is usually a good approximation from well below $5 \mathrm{kHz}$, depending on the channel geometry and propagation conditions.

Eigenrays One of the first reports of the phenomenon of ray chaos is [76]. In a range dependent environment it is known [96] that there exists chaotic ray pairs. Rays from the same point with very small difference in launch angle diverges exponentially with range. This greatly complicates the localization of eigenrays even though this reference shows that it is possible. Other techniques for propagating the rays, by solving a boundary value problem rather than an initial value problem, is reported to make ray chaos scenarios easier to work with [25]. Most of the work reported suggests that the impact of ray chaos in a realistic ocean is only significant at ranges in excess of $100 \mathrm{~km}$. Another characteristic of eigenrays [96], which is also true for the range independent case that we are using here, is that they arrive in clusters. We simplify the ray propagation even more by the way we treat eigenrays. The eigenrays are the rays exactly connecting the transmitter and receiver. The exact eigenrays are not computed in this simulation, but instead a finite number of rays with equal vertical launch angle increments at the transmitter are propagated. All the rays arriving at the receiver range are not contributing to the received acoustic pressure. A rule to decide which rays that should be used is necessary. If they arrive at angles outside the receiver vertical beamwidth the rays are not used, but also the ray arrival depth is significant. The Fresnel zone can be used to determine the limit vertical distance between a ray and the receiver, and currently a rule based on both arrival angle and depth is used. The sector of arrival angles and the range of depths centered at the receiver are supplied externally to the simulator, and the Fresnel zone of a ray can be used as the range of depths where an arriving ray is 
accepted. The rule is that a ray must have both angle of arrival and depth of arrival within the specified ranges in order to be used.

Surface forward scatter The interface to the simulator for surface scattering is by means of en external table with scattering value vs grazing angle. As discussed above there is no sophisticated surface scattering calculation involved, but it can be incorporated by means of the fact that many models yield a forward scattering value vs grazing angle, therefore the only change is to replace the table.

Ray density The physical significance of the extension of a ray is interpreted through the Fresnel zone. The simulator uses an arbitrary ray density, but physically only rays with non-overlapping Fresnel zones are different. Thus shooting many rays within one Fresnel zone gives many rays with essentially similar time-varying attenuation coefficients $h_{i}(t)$. The Fresnel zone for a horizontally traveling ray at range $r=1000 \mathrm{~m}$ in a channel with constant sound speed profile $c=1500 \mathrm{~m} / \mathrm{s}$ for a carrier frequency of $f_{0}=20 \mathrm{kHz}$ is

$$
\mathcal{A}=\sqrt{2 r f_{0} / c}=163 \mathrm{~m}
$$

The Fresnel zone for each ray is not explicitly computed, so there may be a number of rays with very similar attenuation coefficients. This has no impact on the characteristics of the communication channel that is simulated, and cross-ambiguity functions for realizations with different ray densities are not different as long as the major ray paths are present in all the different realizations.

\subsubsection{Simulator examples}

We now look at some examples of simulator output. The absolute travel time is not of interest, and all cross-ambiguity function plots in this section shows differential travel time along the delay axis where the first arriving ray arbitrarily is mapped 
to 10 msec delay. Fig. 3-7 shows an isovelocity sound speed profile with no motion involved. The cross-ambiguity function shows the delay and Doppler resolution of the estimator (given by the signal bandwidth and duration). In this example we take advantage of one very common and powerful way of simplifying the underwater communication channel: The transmitter is not assumed to be omni directional, and its vertical beamwidth is indicated by only tracing rays in the sector shown in Fig. 37. By doing this we avoid later returns from surface and bottom which otherwise could be present depending on the bottom and ocean-surface properties.

The example in Fig. 3-8 shows a realistic ocean sound speed profile taken during summer conditions in the Baltic Sea. We do not present other data from the Baltic Sea, and this sound speed profile is included as a curiosity since the acoustic propagation conditions are very atypical here due to a very low salinity (pike and cod both live together in the Baltic Sea!). The transmitter is moving vertically with 1 $\mathrm{m} / \mathrm{s}$, indicated with the bold arrow. The different rays have different travel times and Doppler shifts, resulting in the spread shown. We note that both positive and negative Doppler components result from this scenario.

The example from outside New Jersey in Fig. 3-9 shows ducted propagation, and this is the way sound travels in the ocean when it propagates over long distances. In this example we have rays with different Doppler shifts that arrive at almost equal times, and this is one form of Doppler spread. The other form of Doppler spread at a single delay occurs when the Doppler shift on a single ray is time-variant.

Fig. 3-10 is another example of such a sound channel at another location and opposite time of the year. This sound speed profile was measured outside Newport (RI) during winter conditions. The attenuation for the rays interacting many times with the ocean-surface and bottom is large, so they are not drawn since they will not contribute to the cross-ambiguity function.

The last example in Fig. 3-11 shows maybe the simplest way of getting Doppler spread. The surface is assumed to be smooth, so there is no Doppler spread from it, 
but the transmitter is moving, so that the projection of its velocity vector onto the different ray launch directions are different. Therefore the different rays get different Dopplers, and the composite response at the receiver is Doppler spread.

Summary We simulate underwater communication channels by means of a timevariant FIR filter. Thus the received signal is a sum of differently delayed and Doppler spread rays. The structure of the FIR filter, i.e., the tap number, location, time evolution and magnitude is given by a ray model of the acoustic field. We compute travel time and attenuation for each ray arriving at the receiver. The rays interacting with the ocean-surface are Doppler spread, and we use the Eckart formulation to get an average reflection coefficient in the specular direction and the Pierson-Moskowitz surface wave spectrum to get the Doppler spread. The raytrace is range invariant and uses piecewise linear sound speed profile. The motion of transmitter, receiver and scatterers is accounted for by allowing different Doppler shifts on different rays. The ocean-surface and bottom attenuation is accounted for by externally supplying values of the attenuation as a function of the grazing angle. The simulator is demonstrated in Fig. 3-7-3-11, and we observe Doppler spread in the cross-ambiguity function of some of these figures. 


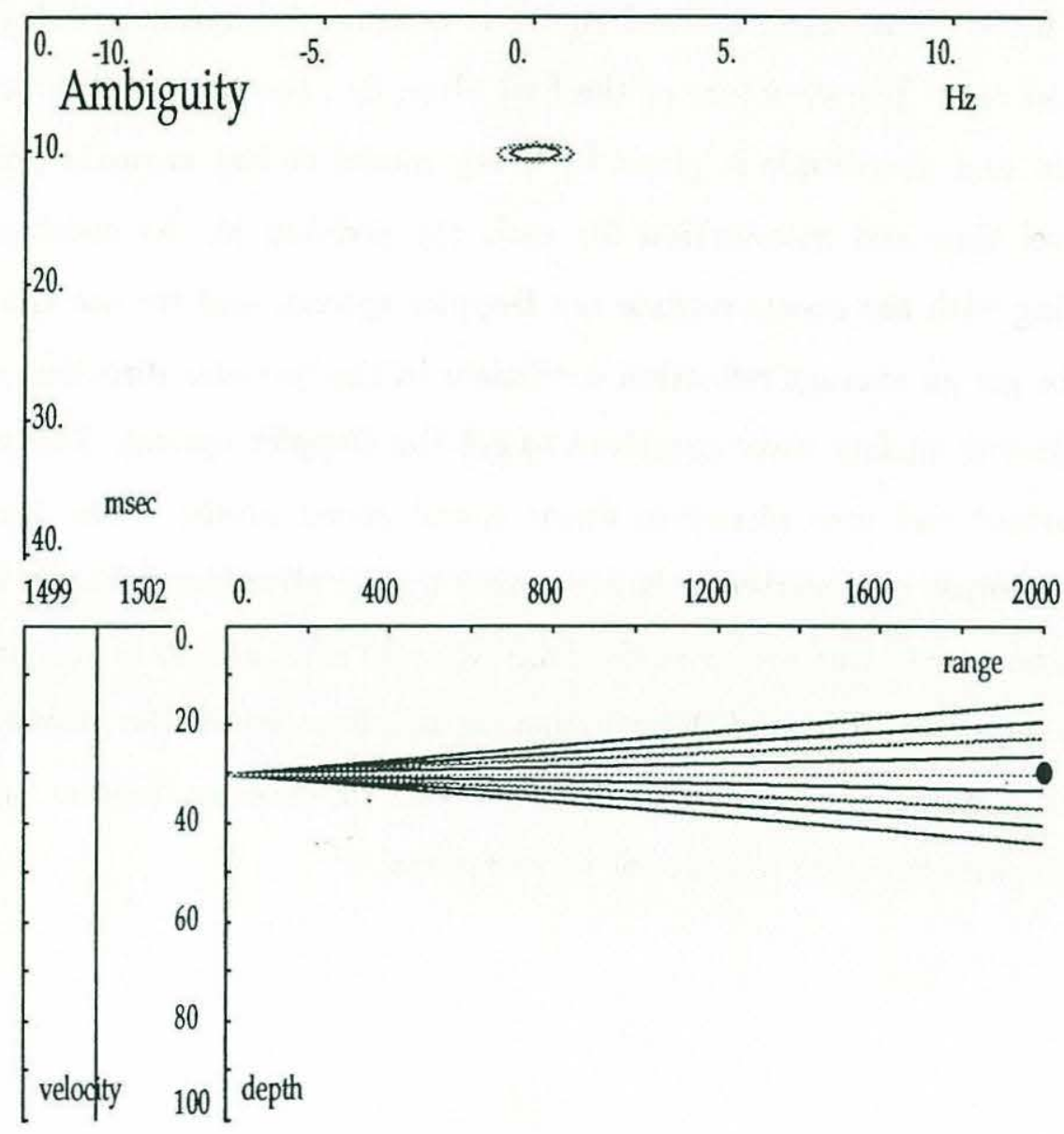

Figure 3-7: Isovelocity example, there is no motion of receiver or transmitter and the vertical beamwidth of the transmitter prevents boundary interaction. The extent of the ambiguity function is given by signal bandwidth and duration. 


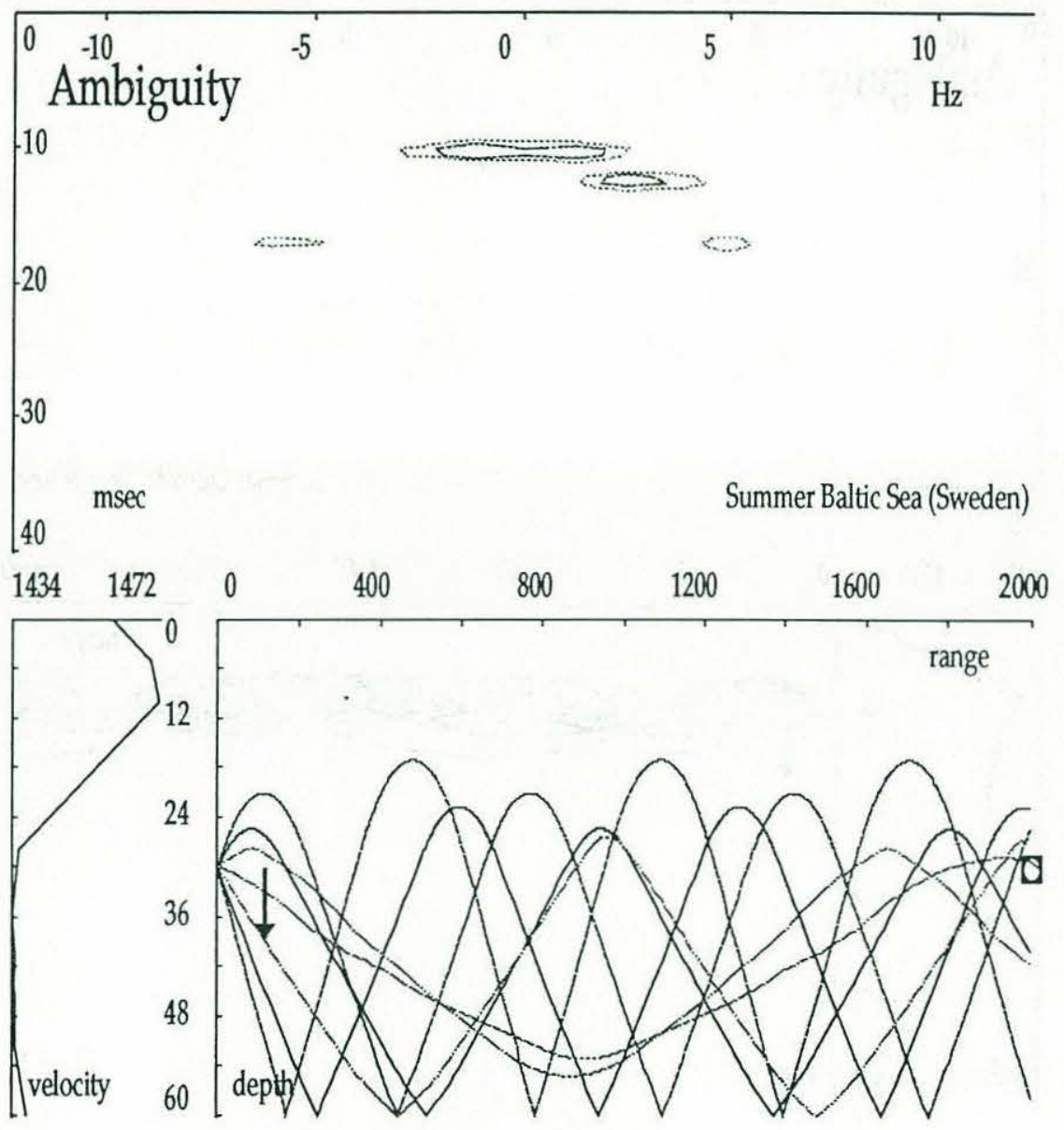

Figure 3-8: Moving transmitter, no receiver motion. 


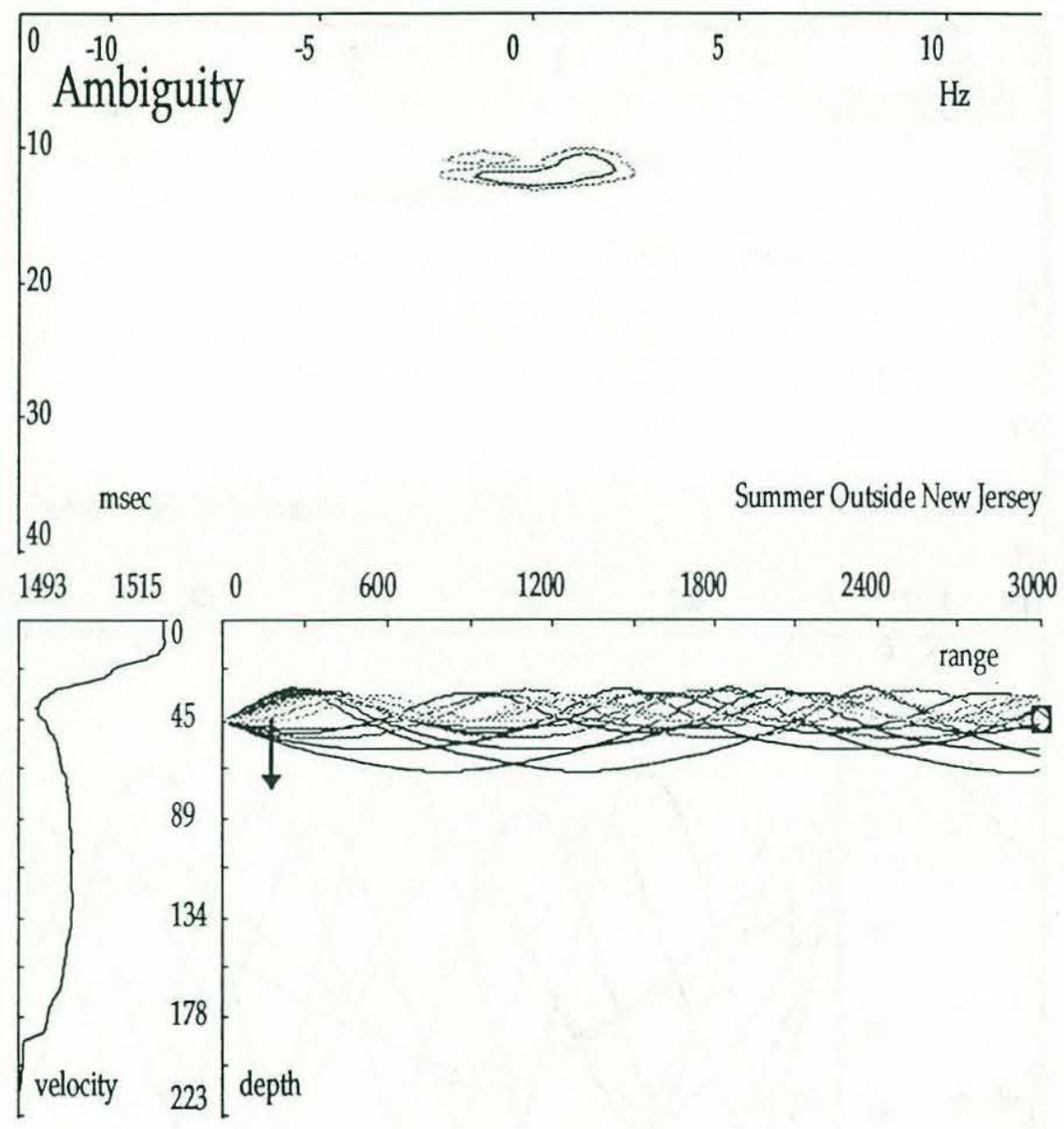

Figure 3-9: Sound speed profile taken off New Jersey during summer conditions. 


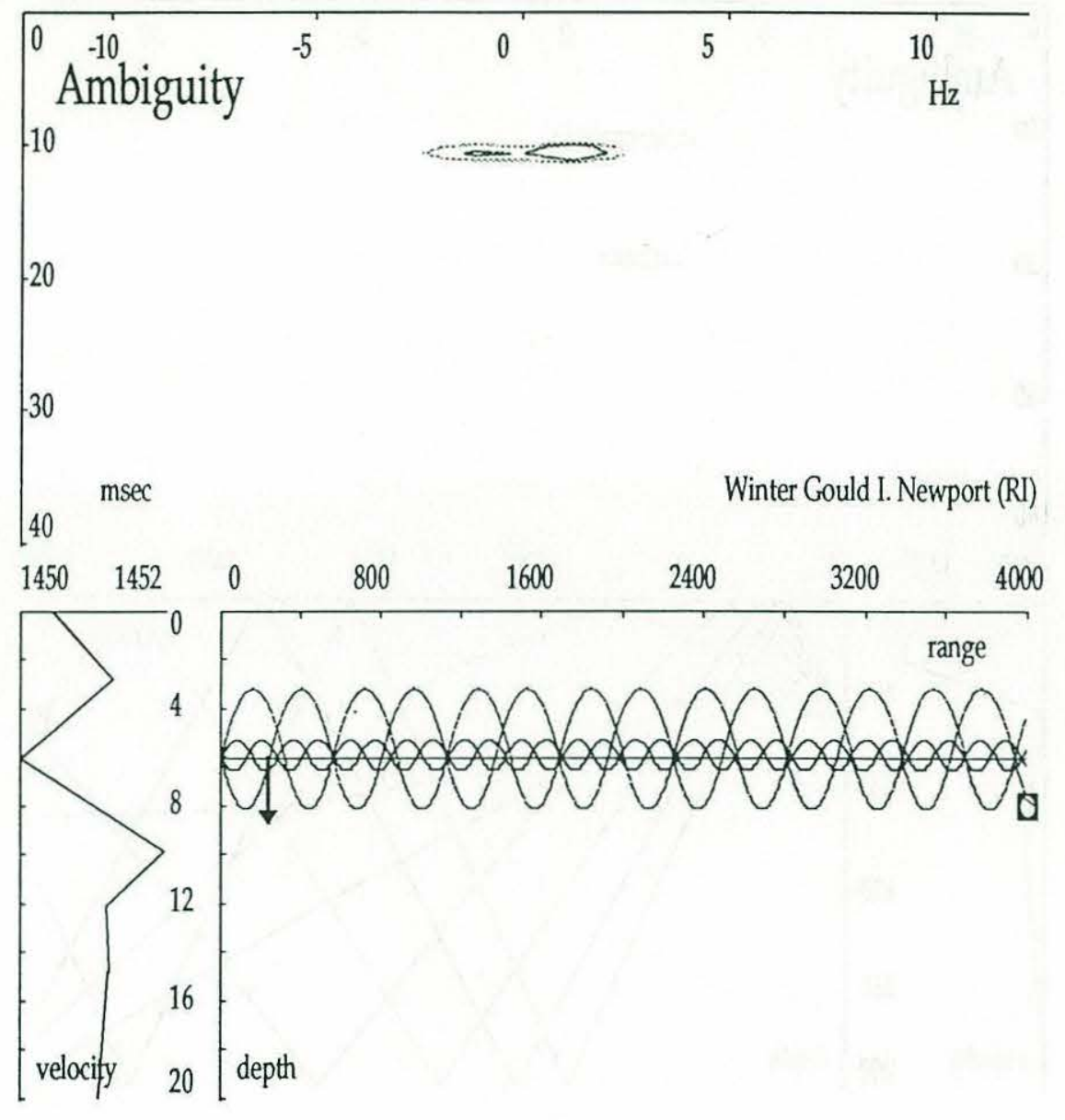

Figure 3-10: Sound speed profile taken outside Newport (RI) during an experiment in the winter. 


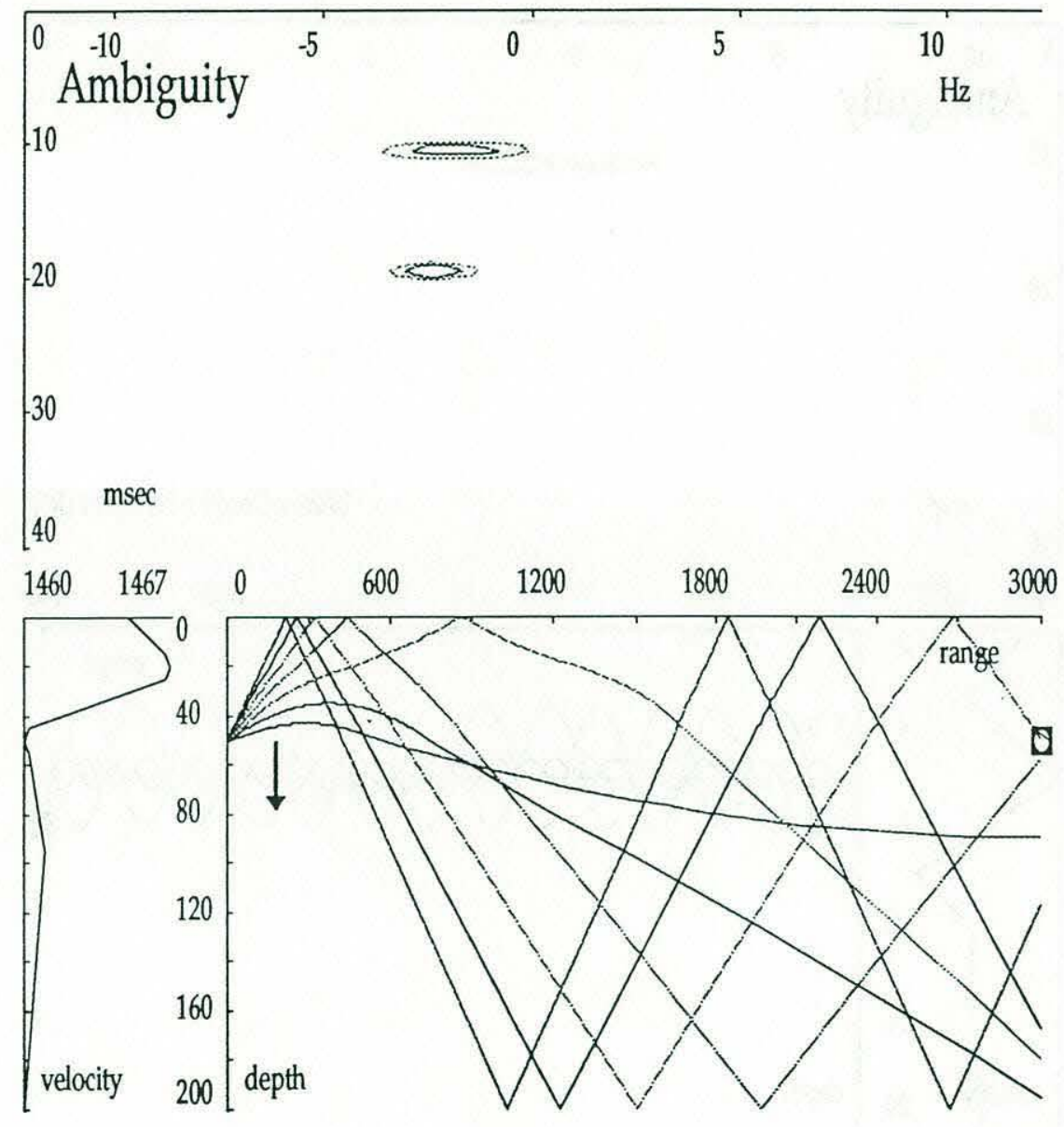

Figure 3-11: An example of a Doppler spread built up of separate Doppler shifts that are different on different ray paths. 


\subsection{Measured scattering functions}

Underwater communication channels are not simply characterized by any canonical form. They vary a lot because such basic quantities as range, water depth and water temperature (giving sound speed profile) impose very drastic changes in the propagation conditions, and these quantities vary over a wide range. In this section we give some examples of this using the cross-ambiguity function to estimate the scattering function and measure the channel. The examples shown are by no means an exhaustive collection of scattering functions, but they serve to demonstrate some of the diversity that underwater communication channels exhibit and how the environment modifies the conditions for communication in a radical way.

In all contour plots, two contour levels that are $3 \mathrm{~dB}$ apart are shown unless something else is explicitly stated. The normalization of the $\mathrm{dB}$ level is relative to the maximum value in each plot. The absolute travel times are not important. The time for the first arrival is merely a normalization issue, and we have not carried out this task. Therefore the first arrival is not shown at $0 \mathrm{msec}$. In the impulse response plots the magnitude axis units are obtained from the receiver number representation, and thus they do not reflect the sound pressure.

\subsubsection{Arctic data}

This experiment was conducted 1993 in an Arctic environment where the sea surface was covered with ice. Thus there was no Doppler spread due to surface motion. The carrier frequency was $15 \mathrm{kHz}$ and the symbol rate was $2500 \mathrm{symb} / \mathrm{sec}$ with quadrature phase shift keying (QPSK) modulation. Data packets consisted of a 1023 symbol long $\mathrm{m}$-sequence that was repeated 6 times. The ambiguity function of the $\mathrm{m}$-sequence is shown in Fig. 3-13 to give an idea of the obtainable resolution in time and frequency. The transmitter and receiver were hanging vertically from the ice cover at a depth of approximately $50 \mathrm{~m}$. Transmissions were available for two ranges: $300 \mathrm{~m}$ and 3 


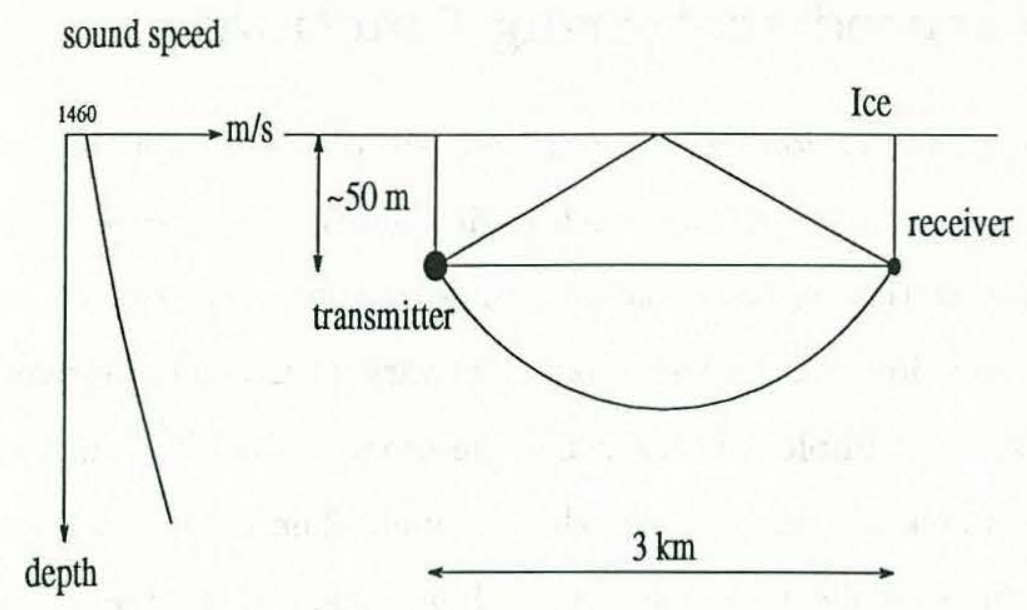

Figure 3-12: The geometry of the Arctic experiment. Both transmitter and receiver were suspended from the ice.

$\mathrm{km}$, and the cross-ambiguity functions of the two channels are shown in Fig. 3-14 and Fig. 3-15 together with impulse response estimates. The second return that is observed in Fig. 3-15 corresponds geometrically (from computing ray travel times) to a surface reflected ray, whereas the third return may be from a deeper refracted ray as suggested in Fig. 3-12 where the geometry is shown. None of the rays are Doppler spread. This scenario essentially gives a sparse delay-spread channel. 


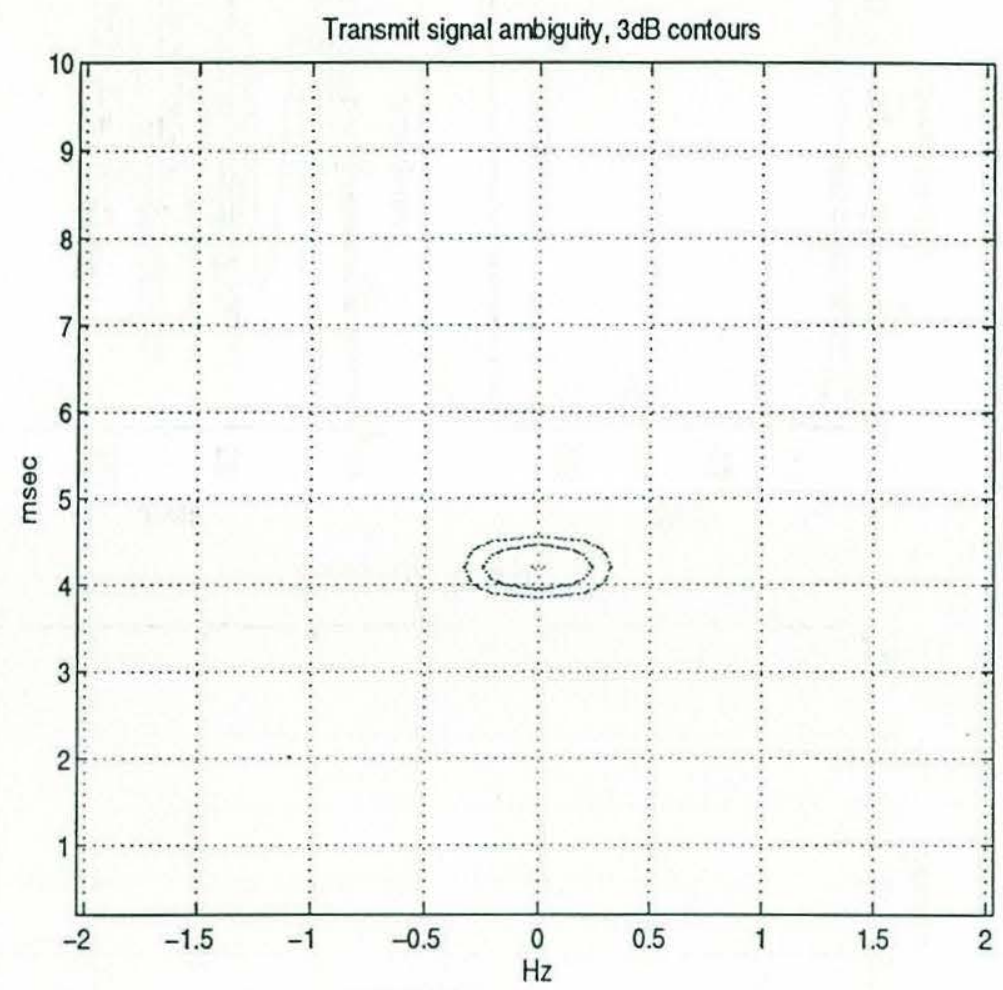

Figure 3-13: The ambiguity function of the transmitted signal. The extent in time is given by the signal bandwidth and the extent in frequency is given by the signal duration. 

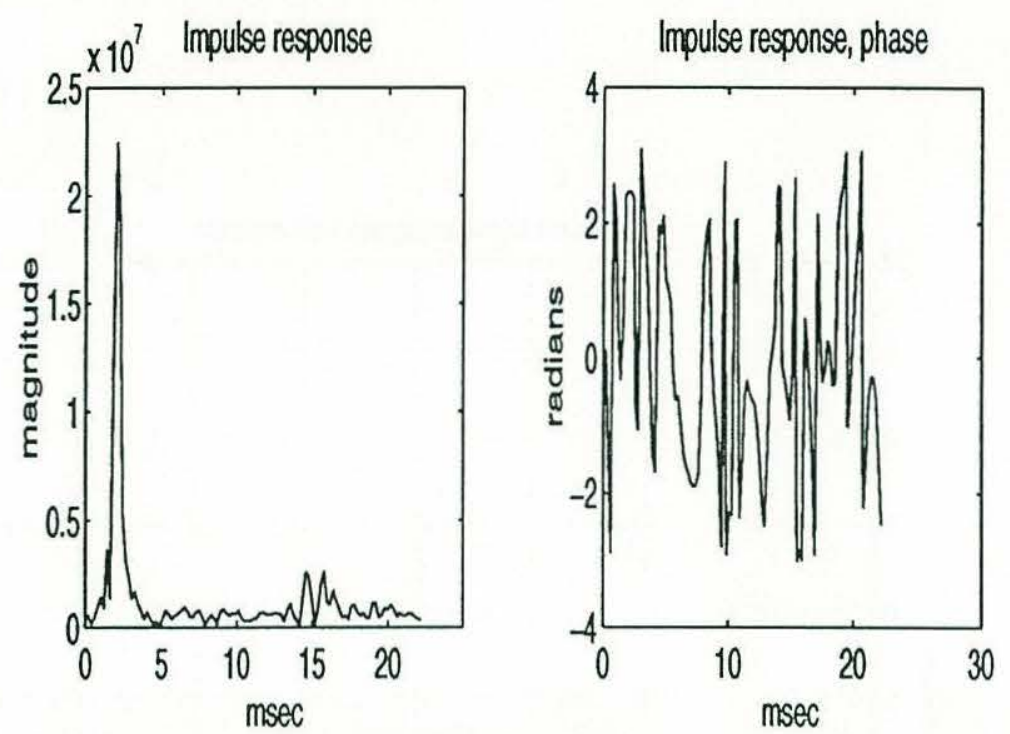

Ambiguity, $3 \mathrm{~dB}$ contours

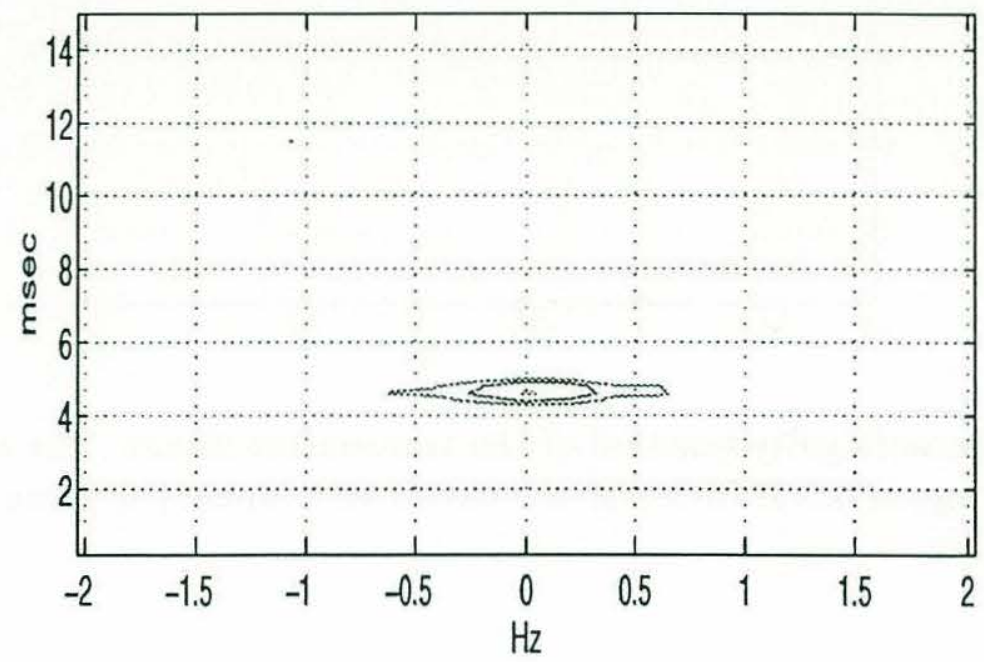

Figure 3-14: Impulse response and channel scattering function for $300 \mathrm{~m}$ range. 

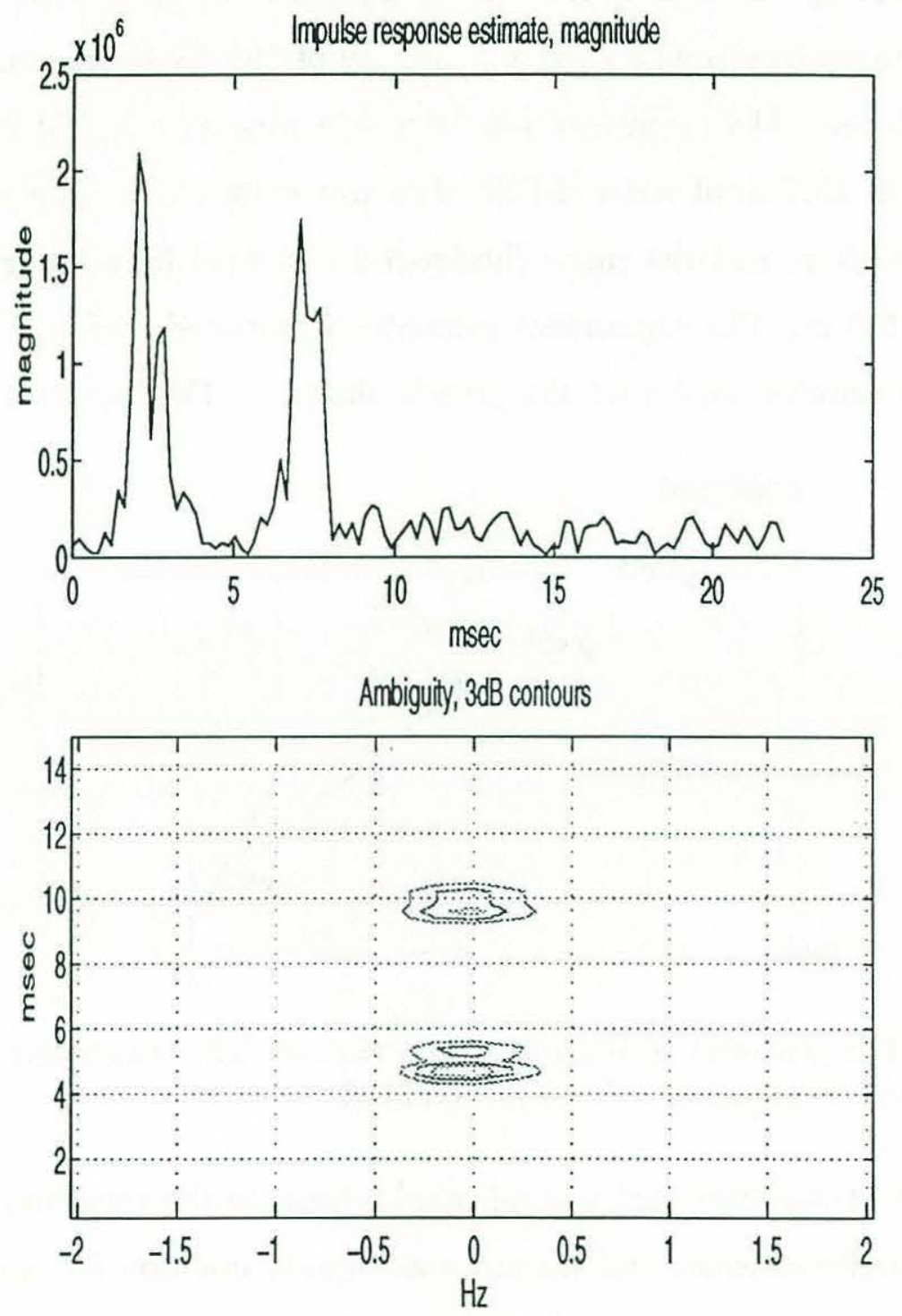

Figure 3-15: Impulse response and channel scattering function for $3 \mathrm{~km}$ range. 


\subsubsection{Autec data}

During the summer of 1994 acoustic transmissions were conducted in deep water outside Florida near the Bahama Islands. It was a several day long experiment and the surface wind speed varied in the range 5-20 knots. In the measurements that we look at the transmitter used a carrier frequency of $15 \mathrm{kHz}$ to transmit QPSK data at $2500 \mathrm{symb} / \mathrm{sec}$. The receiver was bottom mounted at a height of $4.5 \mathrm{~m}$ above the sea floor in $1800 \mathrm{~m}$ of water depth. The transmitter was hanging from a boat at a depth of $15 \mathrm{~m}$, and the range (horizontal distance) between transmitter and receiver was $500 \mathrm{~m}$. The experiment geometry is outlined in Fig. 3-16 where also the empirical summer profile for the area is shown. The transmit sequence was
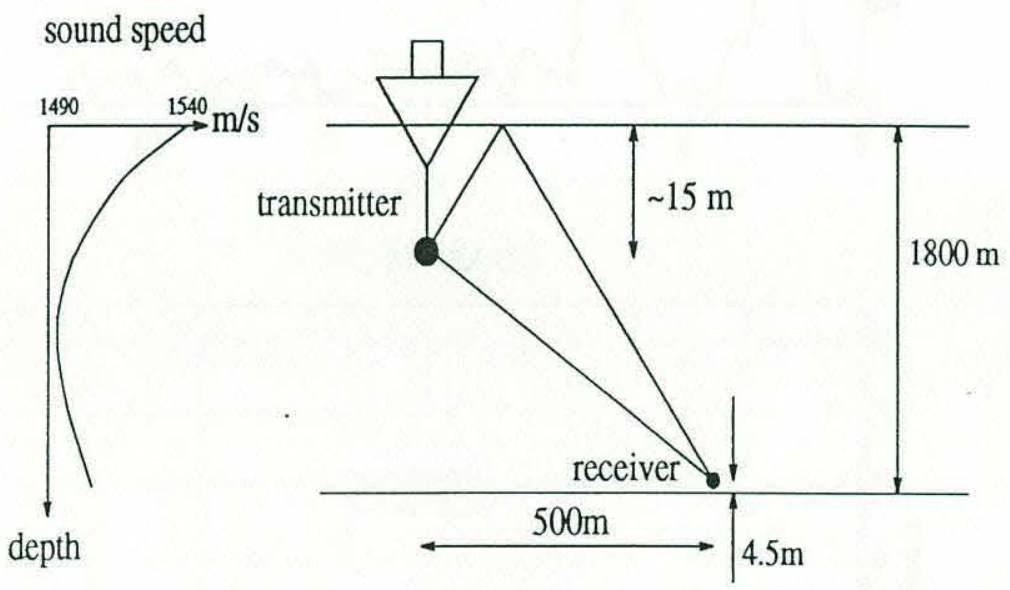

Figure 3-16: The geometry of the Autec experiment. The transmitter is suspended from a boat and the receiver is bottom mounted.

made up of an m-sequence that was repeated 6 times in the same way as the Arctic data. The impulse response and the cross-ambiguity function for one transmission is shown in Fig. 3-17, and in this figure the impulse response estimate is based on 0.2 $\mathrm{sec}$ of data whereas the cross-ambiguity function is computed from $2 \mathrm{sec}$ of data. In this plot we can see a direct path near $(2 \mathrm{~Hz}, 2 \mathrm{msec})$, and also a strongly scattered return starting around $20 \mathrm{msec}$ delay. This second return has consistent delay with a surface reflected path and the wind speed was about 15 knots, so the Doppler- 
spread from the surface is significant. By using (3.10) with this wind speed we get a Doppler spread of $\Delta \nu \approx 11 \mathrm{~Hz}$ which is in good accordance with Fig. 3-17. We observe a mean Doppler shift of the second return which is believed to come from net surface water transportation, since it is known that motion of water particles near a wind driven surface wave field is not purely circular [11]. To further illustrate this a series of scattering function estimates are shown in Fig. 3-18 where the time between adjacent plots is approximately $8 \mathrm{sec}$. We can see that the shift in Doppler of the surface reflected path is always in the same direction consistent with what a steady surface wind would cause. The series Fig. 3-18 demonstrates that Fig. 3-17 is a representative picture of this channel, and by averaging the series of cross-ambiguity functions we obtain the scattering function estimate for this channel.

The difference between Fig. 3-15 and Fig. 3-17 is striking, even though the carrier frequency, bit rate and modulation method is the same the difference in environment gives two very different communication channels. 


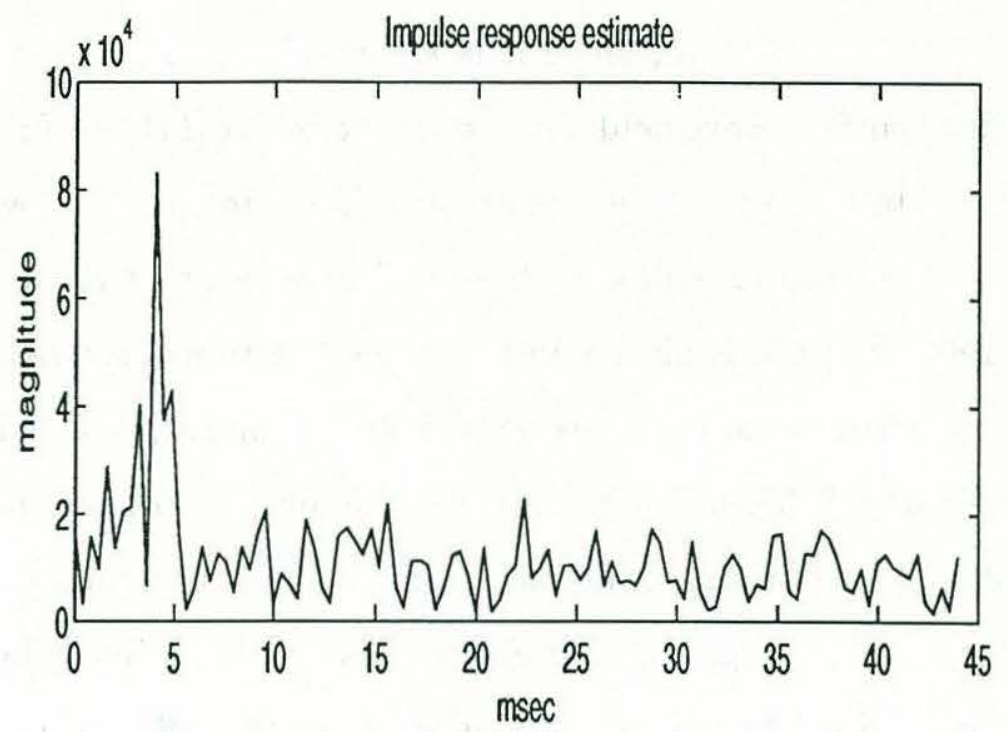

Ambiguity, $3 \mathrm{~dB}$ contours

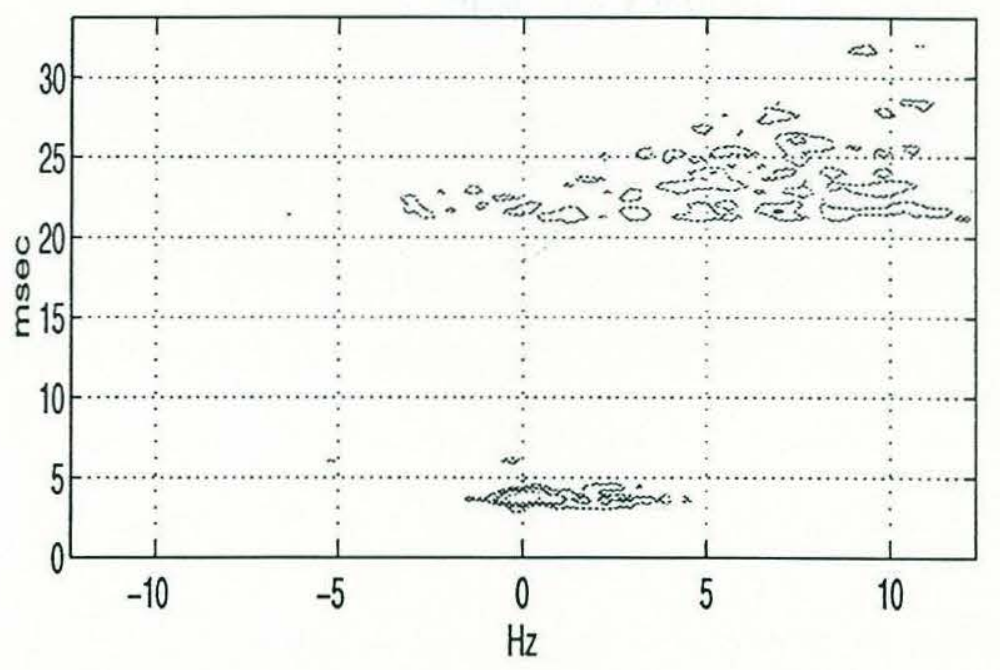

Figure 3-17: Impulse response and scattering function estimates. The channel has a direct path and a surface reflected path that has been Doppler and delay spread due to the surface motion. Three contour levels with distance between contours 3 $\mathrm{dB}$ are shown. 

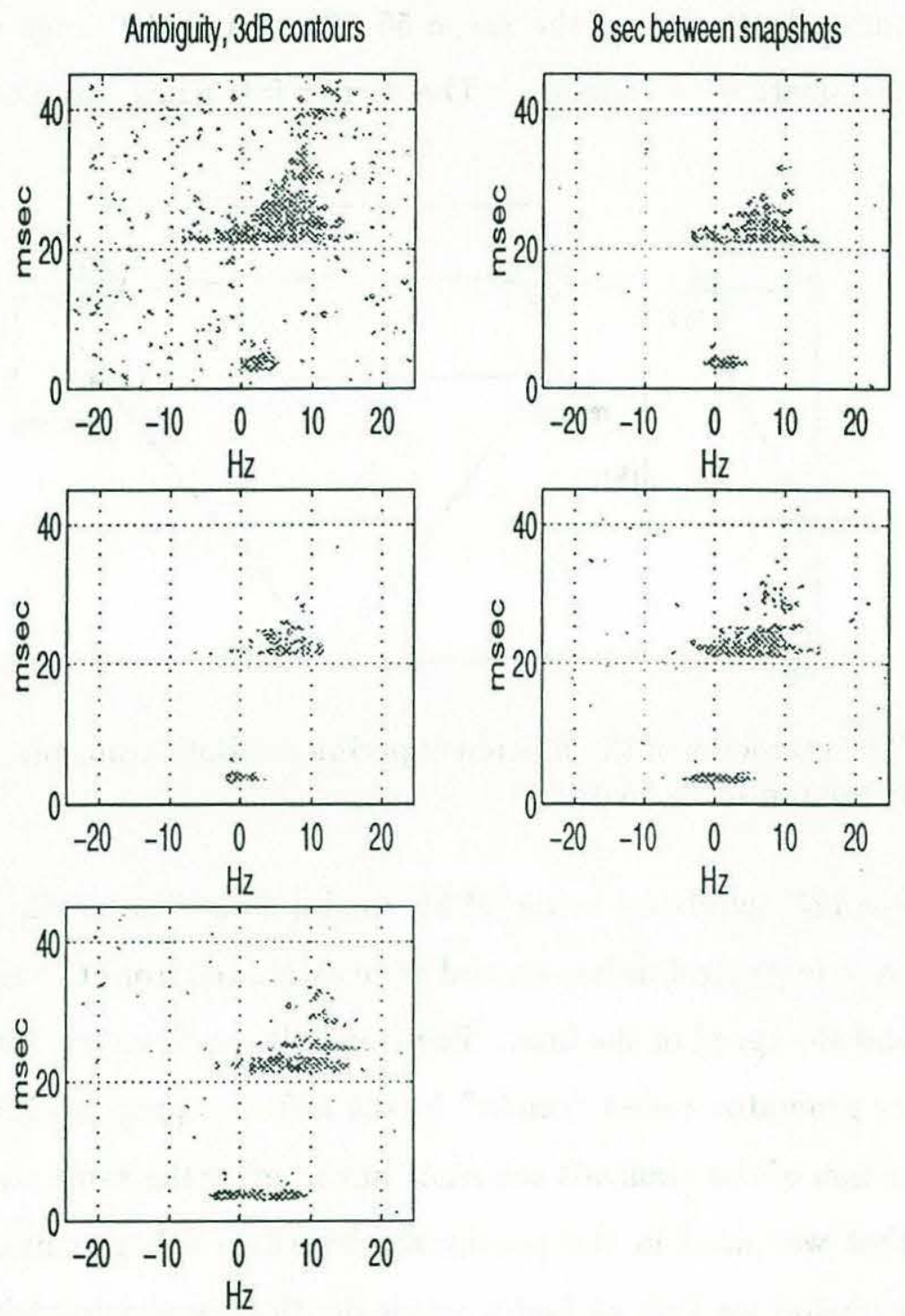

Figure 3-18: Series of cross-ambiguity functions containing the direct path and a surface reflected path that has been Doppler and delay spread due to the surface motion. Three contour levels with distance between contours $3 \mathrm{~dB}$ are shown. 


\subsubsection{Florida data}

In the summer of 1995 an experiment was conducted off Ft.Lauderdale, Florida where both the transmitter and the receiver were on platforms that were towed from boats. The water depth was in the range 50-200 $\mathrm{m}$ and the range was constantly changing as the boats were moving. The carrier frequency was $12.5 \mathrm{kHz}$ and the

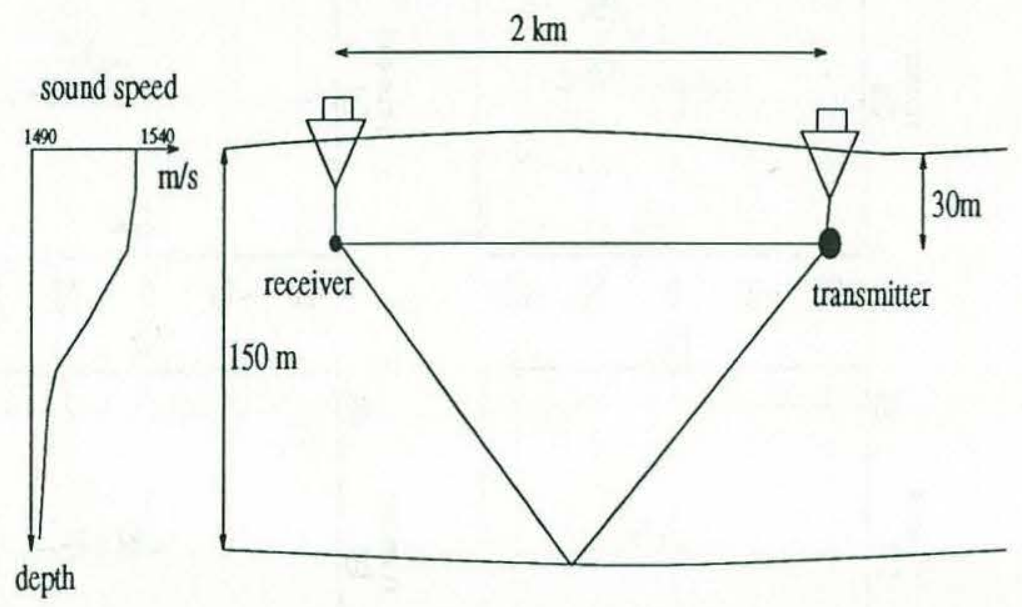

Figure 3-19: The geometry of the Florida experiment. Both transmitter and receiver are suspended from moving boats.

symbol rate was $1250 \mathrm{symb} / \mathrm{sec}$ using QPSK modulation. The depths of transmitter and receiver were in general unknown and were estimated from the amount of cable in the water and the speed of the boat. The transmit sequence was a sequence made with a number generator called "randn" in the software program Matlab, and the ambiguity function of the transmit sequence has roughly the same resolution as the $m$-sequence that was used in the previously described experiments. The site for the first transmission we look at had a water depth of approximately $150 \mathrm{~m}$. The transmitter and receiver depths were $30 \mathrm{~m}$ and the range was $150 \mathrm{~m}$. The two returns shown in Fig. 3-20 correspond to the direct path and a surface return. The shift in Doppler on the surface return relative to the direct path is believed to come from surface motion, and in fact a series of scattering function estimates around the one in Fig. 3-20 shows that the second return moves in Doppler with a time constant on 
the order of a surface swell.

The other transmission is shown in Fig. 3-21 with the geometry of this experiment outlined in Fig. 3-19, and here the boats were approximately $2 \mathrm{~km}$ apart. Of the two returns shown, one is the direct path whereas the other may be a bottom reflected path, but insufficient knowledge about bottom conditions and sound speed velocity profile makes it impossible to know for sure. This channel is another example of an extremely sparse channel. 

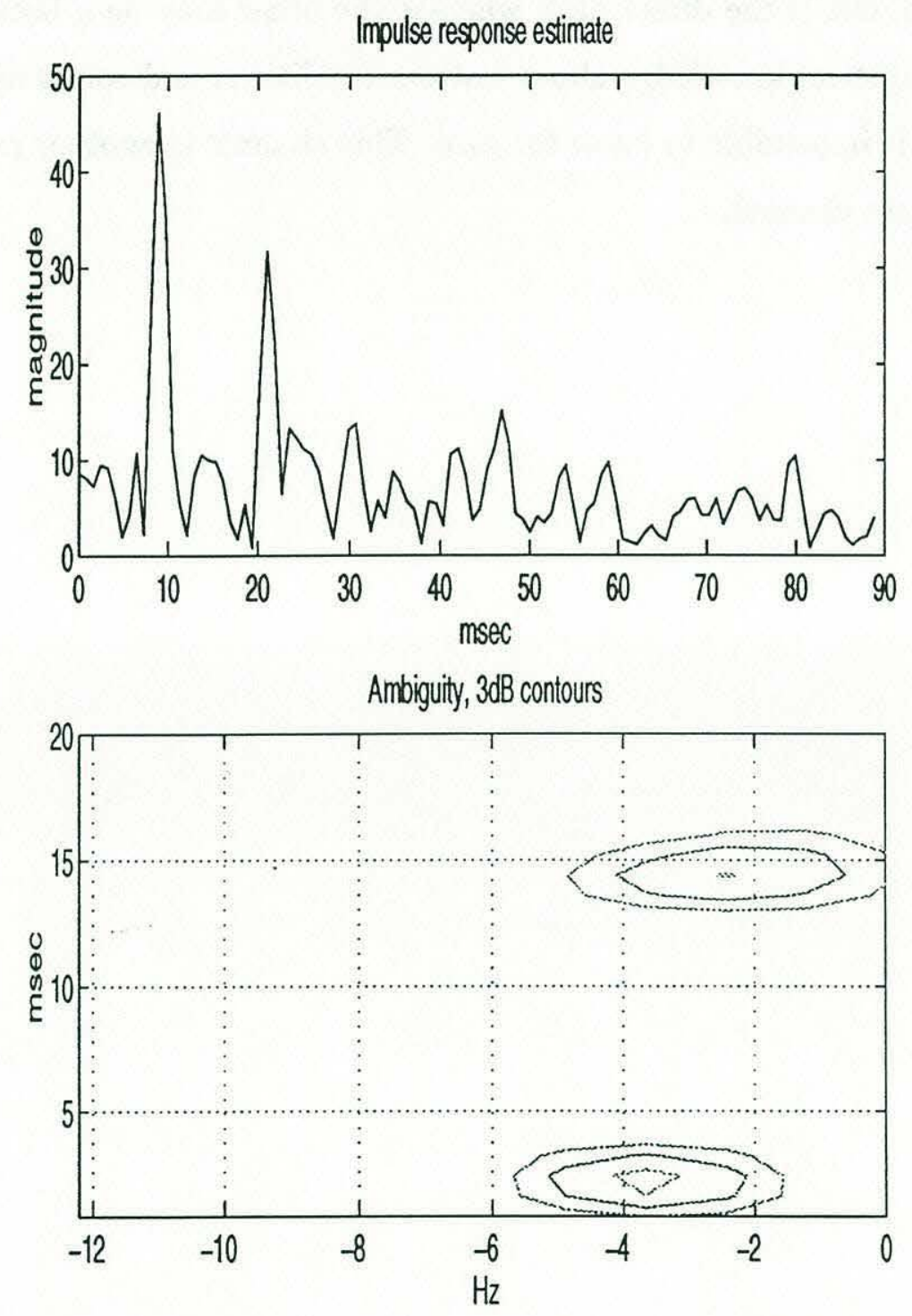

Figure 3-20: The impulse response and channel scattering function for short range $(300 \mathrm{~m})$ data, showing one direct and one surface reflected path. 

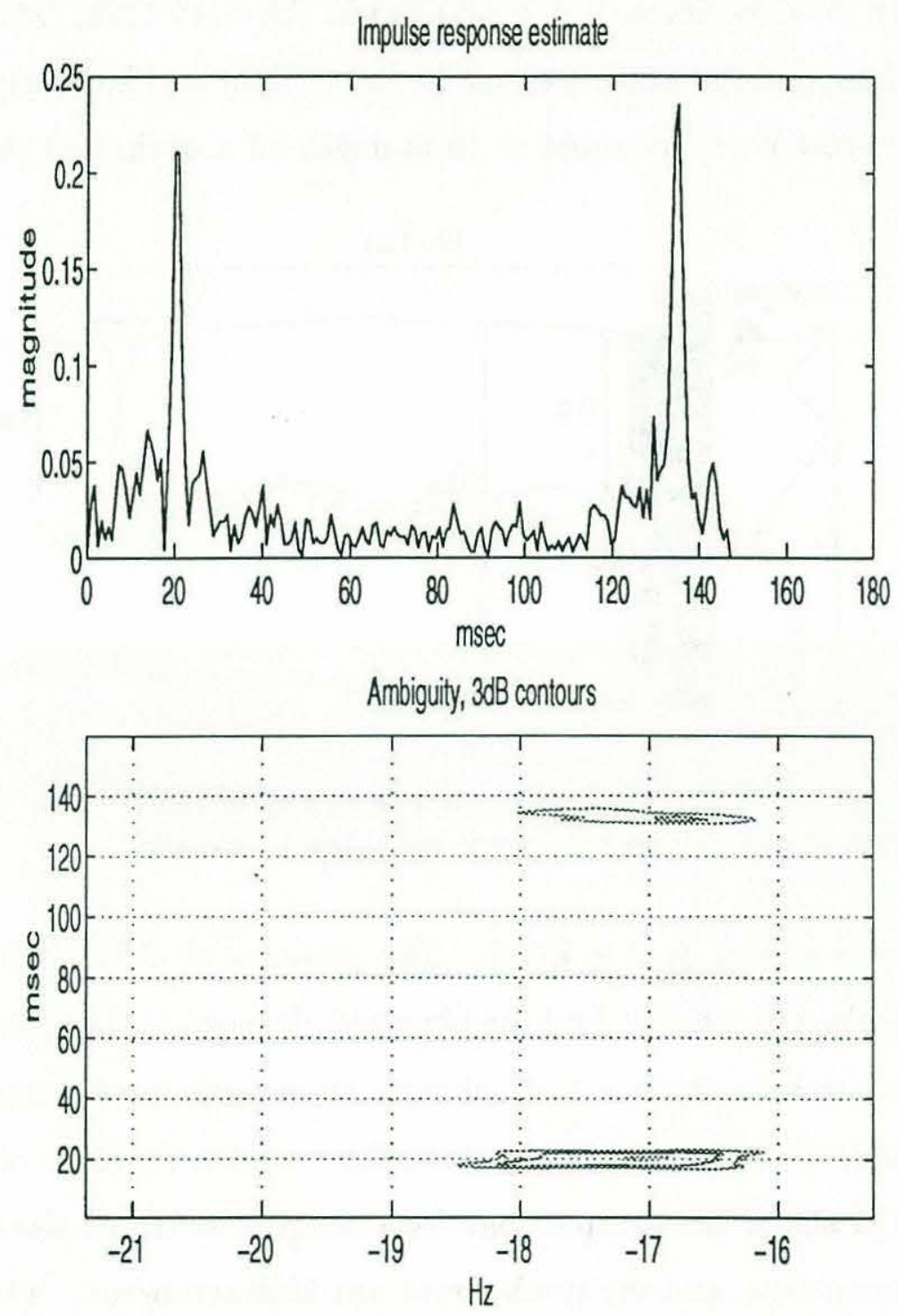

Figure 3-21: The impulse response and channel scattering function for long range (3 $\mathrm{km}$ ) data, showing one direct and one possibly bottom reflected path. 


\subsubsection{Gould Island data}

In an experiment performed in sheltered coastal environment near Newport, Rhode Island symbol rates in the range $600-10000 \mathrm{symb} / \mathrm{sec}$ were used. The modulation was QPSK and the experiment was conducted in February 1996. The water depth was roughly $15 \mathrm{~m}$, and the range was varied from $100 \mathrm{~m}$ to $5 \mathrm{~km}$ during a period of 3 days. The receiver was mounted at $10 \mathrm{~m}$ depth off a dock, and the transmitter

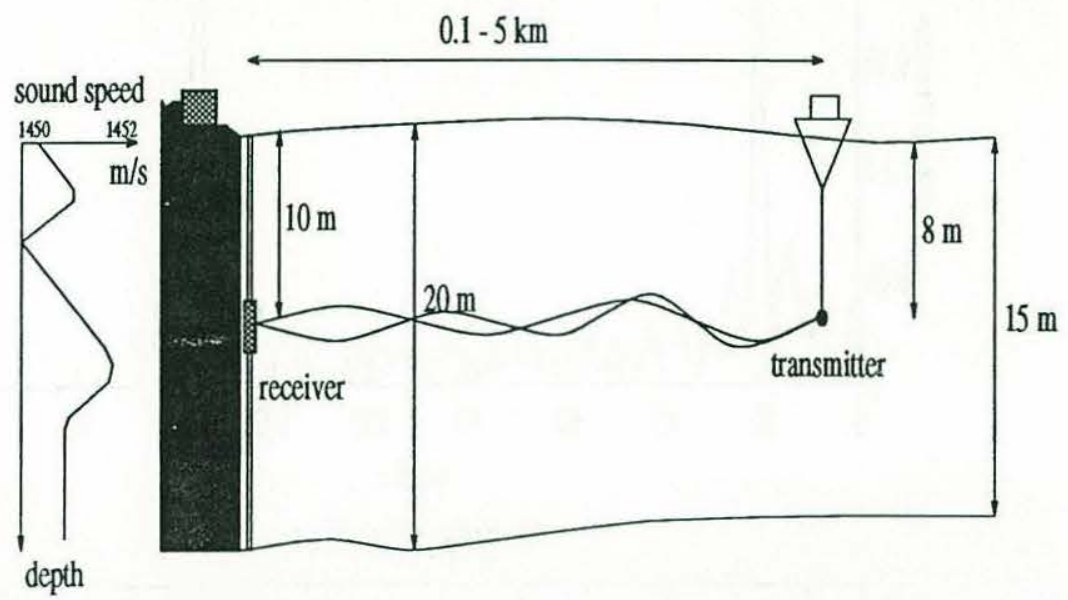

Figure 3-22: The geometry of the Gould Island experiment. The transmitter is suspended from a boat and the receiver is mounted in a dock.

was hanging from a boat at $8 \mathrm{~m}$ depth. The presence of different frequencies on different ray paths can usually best be observed through the scattering function, but during this experiment some transmissions were carried out where the beating of different frequencies also could be seen in the complex envelope of the received signal. Fig. 3-23 shows the complex envelope sampled at 2 samples/symbol from one of the transmissions, and the symbol rate was $2500 \mathrm{symb} / \mathrm{sec}$. The lower panel shows the complex envelope of a signal obtained with the simulator in a scenario with two dominant ray paths and a vertically moving transmitter with different Doppler shifts on each of the paths. Since the ray path directions have different projections onto the transmitter movement direction they get different Doppler shifts. It is believed that the beating of the two paths creates the periodic pattern seen in the 
real data in the same way as it creates it in the simulated data. The beat frequency in this case is so slow (it corresponds to a Doppler spread of $0.8 \mathrm{~Hz}$ ) that it is not a significant problem for a conventional receiver to decode.

Fig. 3-24 shows a transmission with more Doppler spread, and the geometry of this transmission is outlined in Fig. 3-22 The range in this case was $4 \mathrm{~km}$, and the symbol rate was $600 \mathrm{symb} / \mathrm{sec}$. From varying the transmitter depth carefully and monitoring the received power level at the receiver it was obvious that there was a sound channel present. By moving the transmitter 1-2 m vertically a difference of $10 \mathrm{~dB}$ in received power was observed. The sound channel was later verified by processing of sound speed measurements that were taken in the area the same time. The net Doppler shift is explained from that the boat was drifting, and the two peaks in the cross-ambiguity function giving a spread may be from different rays with different launch angles. The set of transmissions represented by Fig. 3-24 has not been decoded with any conventional single data channel receiver, even though the SNR is around $15 \mathrm{~dB}$.

Summary We present data açquired from the ocean at four different locations varying from the Arctic ice covered ocean to warm water south of Florida. We observe a wide range of underwater communication channels varying from not spread in Fig. 3-14 via pure delay spread in Fig. 3-15 and pure Doppler spread in Fig. 3-24 to doubly spread in Fig. 3-18 and Fig. 3-20. We also observe the time-varying magnitude of the complex envelope in Fig. 3-23 illustrating rays with different Doppler shifts. 
Real data, Gould Island 1996

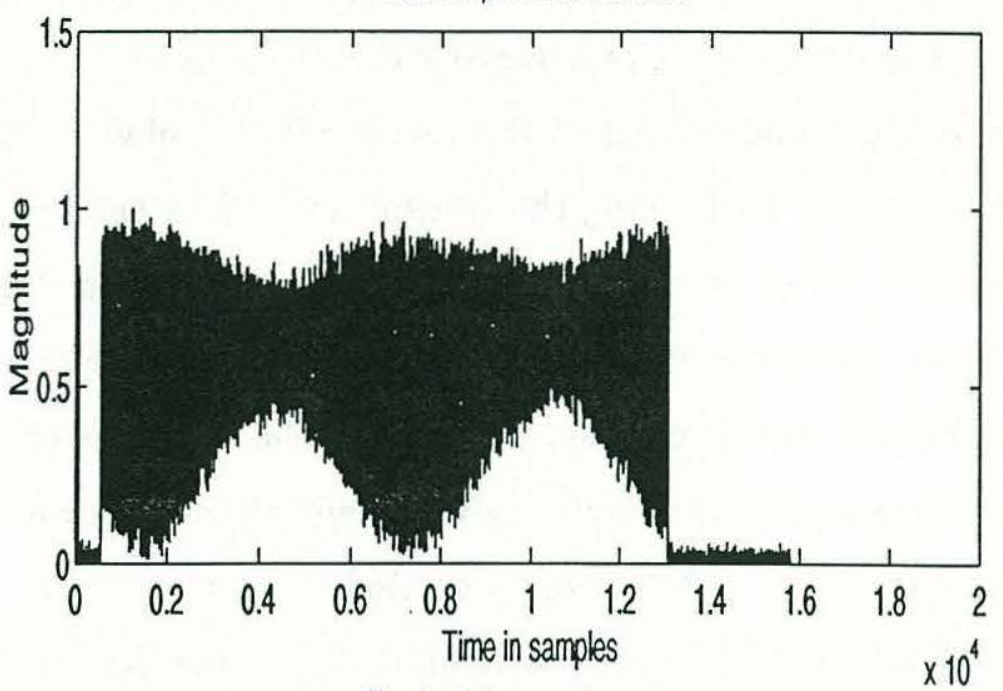

Simulated data, moving source

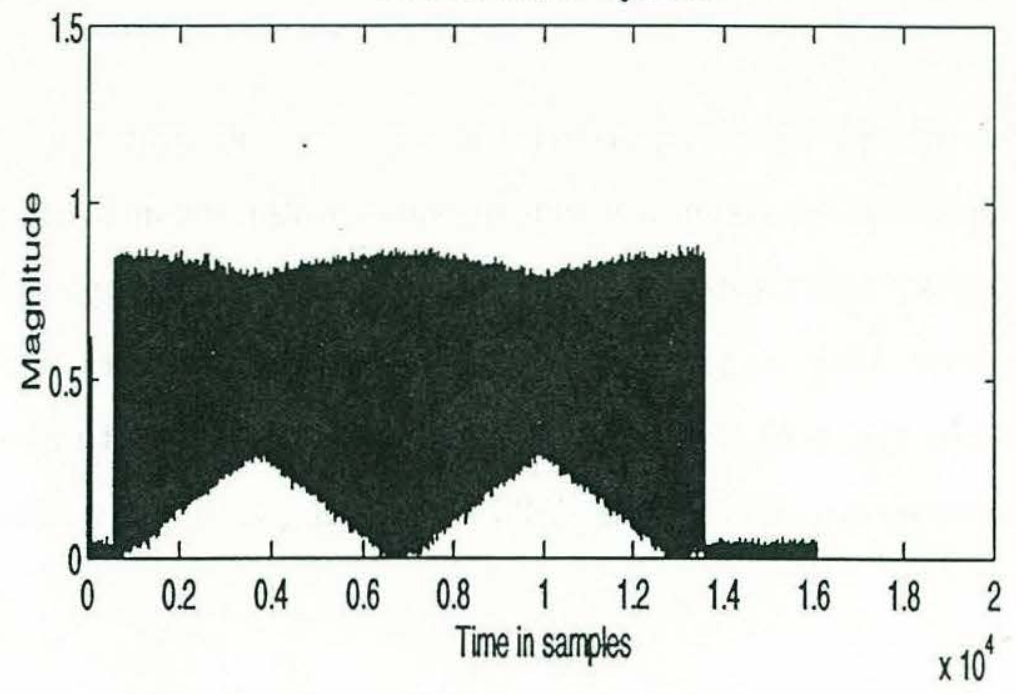

Figure 3-23: The complex envelope of real and simulated data. Two paths with different Doppler shifts create a beating frequency. 


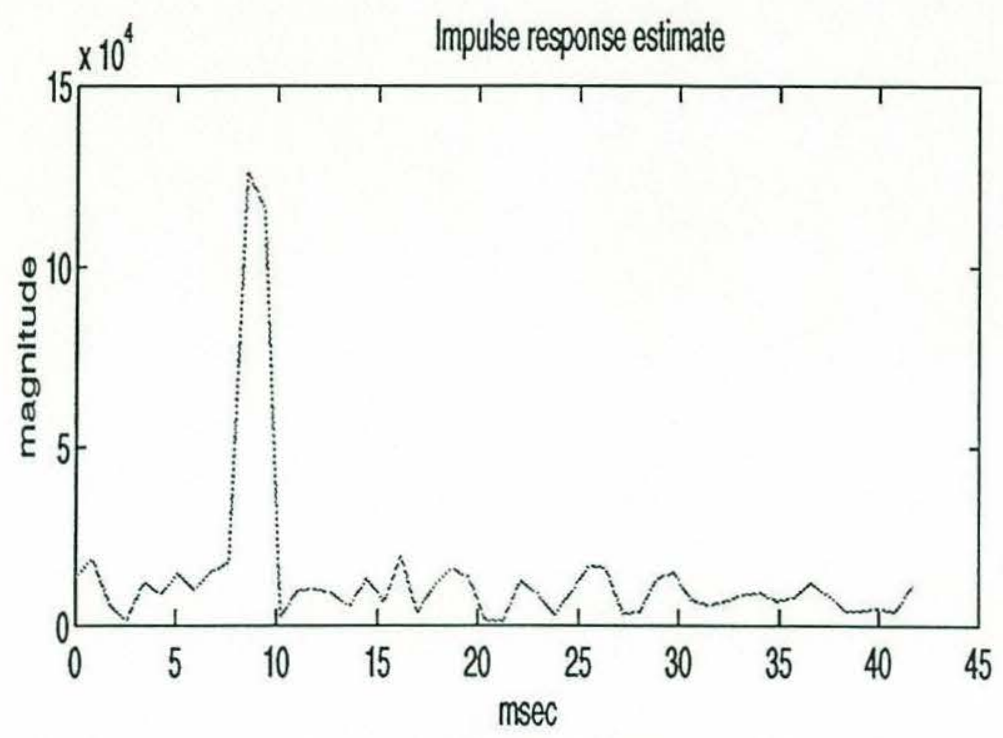

Ambiguity, $3 \mathrm{~dB}$ contours

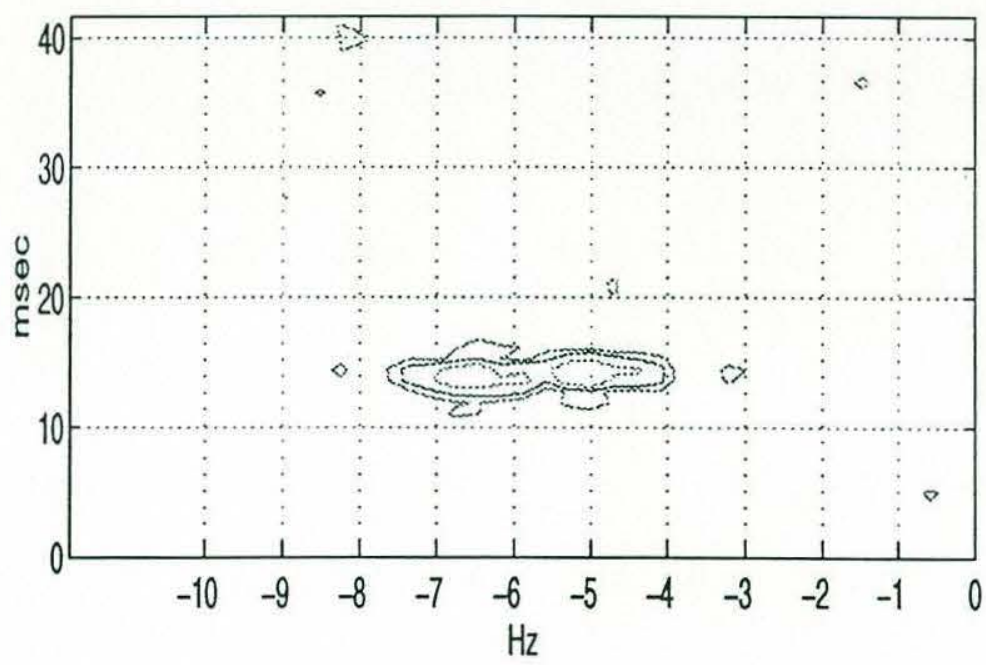

Figure 3-24: Impulse response and channel scattering function of Doppler spread data from transmitter movement. Three contour level with spacing $3 \mathrm{~dB}$ are shown in the lower panel. 
. 


\section{Chapter 4}

\section{Receiver}

\subsection{Maximum likelihood receiver}

\subsubsection{Preview and motivation}

The best quality criterion for a communication system is the probability that a decoding error takes place. If statistical models are imposed on the noise and signal this probability can be computed. The probability of error is therefore often used as a design criterion and it is well known that minimum probability of error decoding is the same as maximum aposteriori (MAP) decoding [40], [82]. When the transmitted symbols are equally likely, and the channel response is known, maximum aposteriori (MAP) decoding is the same as maximum likelihood (ML) decoding. In a realistic situation both the transmitted sequence and the channel response are unknown, and they would have to be estimated. The major point of this section is to show that, in the case of the underwater communication channel, the ML receiver is very computationally intensive even in the case of known channel response. This motivates the choice of the suboptimal minimum mean square error (MMSE) receiver that is introduced in Section 4.3. We assume that the channel response $U_{l, k}$ is given in the derivations of Section 4.1.2-4.1.4, and we bear in mind that the channel iden- 
tification and tracking should be performed simultaneously with the decoding in a realistic receiver. This is the way it is carried out when we discuss the decision feedback equalizer and the time updated RLS (TU-RLS) receiver in Section 4.2 and Section 4.3. The requirement of estimating the channel makes it necessary to have two receiver modes of operation called training and tracking mode. We qualitatively discuss the factors that determine the channel estimation error after the presentation of the ML receivers, and we return to a quantitative discussion of the channel estimation error during training and tracking in Section 4.3.6 and Section 4.3.8.

\subsubsection{Doubly spread channel}

We use the delay-Doppler-spread function $U(\tau, \nu)$ to represent time and frequency dispersive channels, where we remember that $U(\tau, \nu) d \nu d \tau$ is the complex scattering amplitude of the signal at delay $\tau$ and Doppler shift $\nu$. We now transmit the signal $x_{0}(t)$ represented by the complex envelope $x(t)$, and we receive $y_{0}(t)$ which can be represented by its complex envelope

$$
y(t)=\iint x(t-\tau) U(\tau, \nu) e^{j 2 \pi \nu t} d \nu d \tau+w(t)
$$

where $w(t)$ is zero-mean complex Gaussian white noise. By using the discrete representation (2.56) we have

$$
y(n)=\sum_{l, k} U(l, k) z(n-l) e^{j 2 \pi k \Delta \nu n}+w(n)
$$

where $z(n)$ is the transmitted data sequence and $w(n)$ has variance $\sigma_{w}^{2}$. The ML estimate of $\mathbf{z}=[z(n) \cdots z(n+L-1)]$ is given by means of the conditional probability density $p(\mathbf{y} \mid \mathbf{z}, U)$ where $\mathbf{y}=[y(n) \cdots y(n+N-1)]$ and $U$ is the set of all coefficients 
$U(l, k)$. This distribution is Gaussian and given by

$$
\begin{aligned}
p(\mathbf{y} \mid \mathbf{z}, U) & =\left(\frac{1}{\pi \sigma_{w}^{2}}\right)^{N} \exp \left(-\sum_{m=n}^{n+N-1} \mid y(m)-\sum_{l, k} z(m-l) U(l, k)\right. \\
& \left.\times\left. e^{j 2 \pi k \Delta \nu m}\right|^{2} / \sigma_{w}^{2}\right)
\end{aligned}
$$

Therefore, remembering that the channel response $U_{l, k}$ is considered known, we find the ML estimate by performing

$$
\min _{\mathbf{Z}} \sum_{m=n}^{n+N-1}\left|y(m)-\sum_{l, k} z(m-l) U(l, k) e^{j 2 \pi k \Delta \nu m}\right|^{2} .
$$

We comment on this estimator after the section below on delay spread.

\subsubsection{Delay spread channel}

When the channel is delay spread only we have a time-invariant channel which gives intersymbol interference (ISI). The ML receiver for this channel is a well known result [82], [100], and it can be viewed as a special case of the result from Section 4.1.2. The channel is now characterized by its impulse response $h(\tau)$ and we can relate the delay-Doppler-spread function and the impulse response $h(\tau)$ by

$$
U(\tau, \nu)=h(\tau) \delta(\nu)
$$

where $\delta(\nu)$ is the Dirac delta function, and by means of this (4.1) yields

$$
y(t)=\int x(t-\tau) h(\tau) d \tau+w(t) .
$$

By using the discrete representation (2.56) we get

$$
y(n)=\sum_{l} z(n-l) h(l)+w(n) .
$$


The probability density for $\mathbf{y}$ conditioned on the transmit sequence $\mathbf{z}$ and the set $h$ containing all $h(l)$ is

$$
p(\mathbf{y} \mid \mathbf{z}, h)=\left(\frac{1}{\pi \sigma_{w}^{2}}\right)^{N} \exp \left(-\sum_{m=n}^{n+N-1}\left|y(m)-\sum_{l} z(m-l) h(l)\right|^{2} / \sigma_{w}^{2}\right)
$$

and the ML receiver is obtained by maximizing $p(\mathbf{y} \mid \mathbf{z}, h)$. This is the same as minimizing the exponent of (4.8), which is the same as minimizing

$$
J(\mathbf{z})=2 \operatorname{Re}\left[\sum_{m=n}^{n+N-1} y^{*}(m) z(m-l)\right]-\sum_{m=n}^{n+N-1} \sum_{l, i} z(m-l) z^{*}(m-i) h(l) h^{*}(i)(.
$$

One commonly used method for minimizing $J(\mathbf{z})$ is the Viterbi algorithm, but when the information symbols are M-ary, and the ISI spans L symbols, $M^{L}$ probabilities must be computed for each decision. A realistic pair of impulse response duration (taken from the ocean) and data rate may be $(100 \mathrm{msec}, 2500 \mathrm{symb} / \mathrm{sec})$. If the modulation is QPSK in this case we get

$$
M^{L}=4^{250}
$$

error probability computations for each decoded symbol. This is a very high computational load, and it makes this approach less attractive.

It is now clear that in the case of the doubly spread channel, that was treated in Section 4.1.2, the computational load associated with the ML receiver for this channel is even higher. 


\subsubsection{Doppler spread channel}

We now consider the other special case of the doubly spread channel which is the purely Doppler spread channel. We can write the delay-Doppler-spread function as .

$$
U(\tau, \nu)=F(\nu) \delta(\tau)
$$

and from (4.1) we get

$$
y(t)=\int x(t) F(\nu) e^{j 2 \pi \nu t} d \nu+w(t) .
$$

By using (2.56) we get

$$
y(n)=z(n) \sum_{k} F(k) e^{j 2 \pi k \Delta \nu n}+w(n) .
$$

The probability density for $y(n)$ conditioned on $z(n)$ and the set $F$ of all $F(k)$ is Gaussian and given by

$$
p(y(n) \mid z(n), F)=\frac{1}{\pi \sigma_{w}^{2}} e^{-\left|y(n)-z(n) \sum_{k} F(k) e^{j 2 \pi k \Delta \nu n}\right|^{2} / \sigma_{w}^{2}} .
$$

The ML receiver for $z(n)$ is obtained by minimizing the exponent of (4.14) which yields

$$
\hat{z}(n)=\min _{z(n)}\left|z(n)-\frac{y(n)}{\sum_{k} F(k) e^{j 2 \pi k \Delta \nu n}}\right| .
$$

This amounts to dividing the current sample $y(n)$ with a complex gain and then choosing the closest symbol. A receiver implementing (4.15) corresponds to the IFS Doppler line that is discussed in Section 2.4 because, referring to Fig. 2-7, in this case $y(n)$ is the input signal whereas $\hat{z}(n)$ is the output signal. This receiver is not robust to modeling errors, since there is nothing that prevents the dividing gain from 
being arbitrarily close to zero and this gives noise enhancement.

By using the concept of duality that was discussed in Section 2.4 we observe that the dual of the receiver in (4.15), which would apply to the dual channel, is an IIR delay line. The dual channel is the delay spread (LTI) channel, and the dual of the receiver in (4.15) is the zero forcing equalizer [82]. This equalizer has the problem of noise enhancement when the delay spread channel has a spectral null. In the same way the IFS receiver in (4.15) has the problem of noise enhancement when the Doppler spread channel, which is the dual of the delay spread channel, has a fade. A fade is a temporal null which is the dual of a spectral null. One way of avoiding noise enhancement in the case of the delay spread channel is to constrain the receiver to be a FIR delay line instead of using the ML criterion that yields the IIR delay line. Thus it is straightforward by the concept of duality to motivate the constraint in the case of a Doppler spread channel: We can use a FFR Doppler line in order to avoid the noise enhancement in the case of a channel fade. This direction is utilized in Section 4.3.4 when we present a receiver for the Doppler spread channel. It is useful for the underwater communication channel when we have different rays that have the same travel time and different Doppler shifts.

Summary The discrete representation in Section 2.3.2 is used to find the ML receiver in the case of doubly spread (4.4), delay spread (4.9) and Doppler spread (4.15) channel assuming the channel response is known. The computational load in the case of a delay spread channel for a realistic underwater communication channel is extensive (4.10) and even higher in the case of a doubly spread channel.

\subsubsection{Training vs tracking}

Motivation The ML receivers in Section 4.1 all rely on a known channel response. In practice they are used with a channel response that is derived simultaneously with the decoding, and the decoding error rate depends on the channel estimation 
error. Before the receiver can start decoding it is initialized with a channel response estimate which is obtained from a training sequence, and this is referred to as training mode. This estimate is maintained during decoding, and this is referred to as tracking mode. We now discuss the factors that determine the channel estimate both in training and tracking mode. In order to synchronize the receiver and transmitter, a short sequence, usually a Barker sequence, is transmitted a fixed time before the training symbols. The receiver runs a filter matched to this sequence and thereby obtains synchronization. We show the total transmission of one packet in Fig. 4-1.

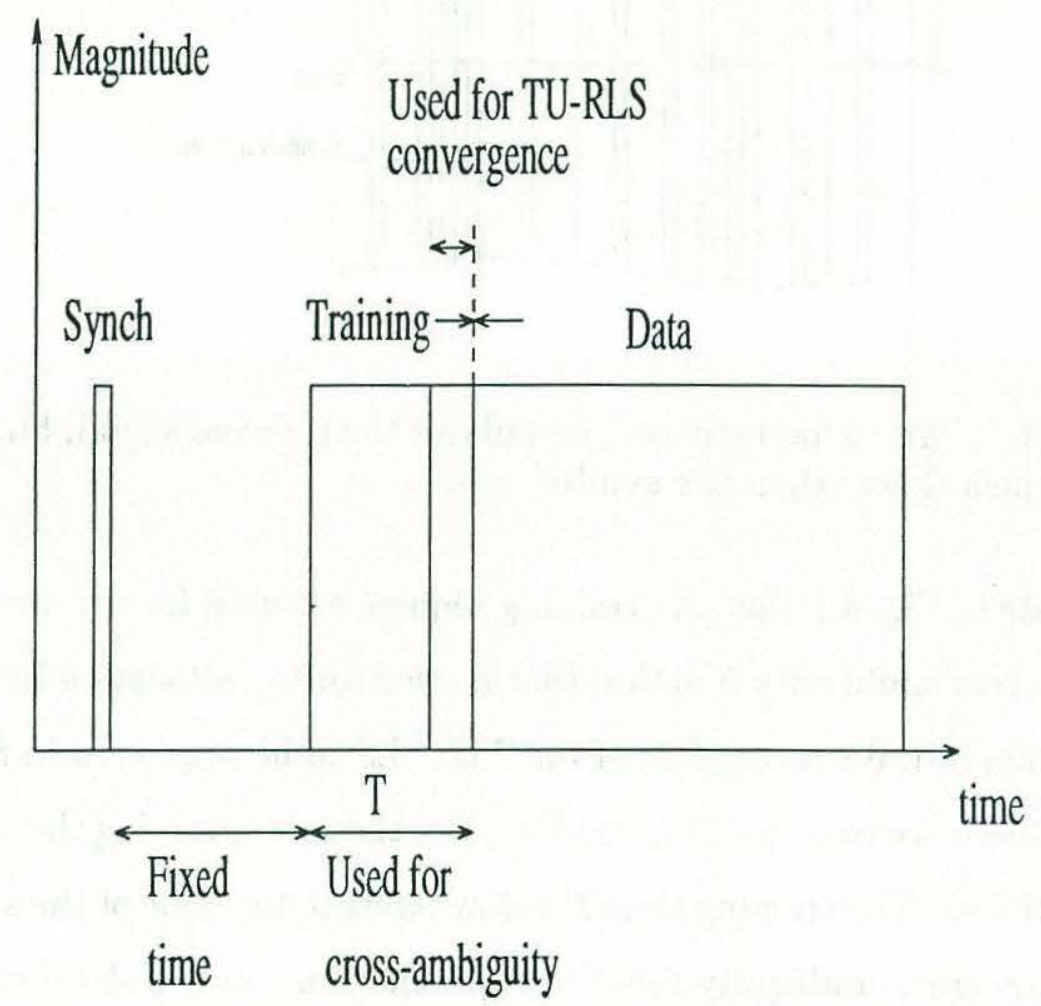

Figure 4-1: Data packet format containing synchronization, training sequence used for the cross-ambiguity function and the TU-RLS convergence and data symbols to be decoded.

We find during the discussion of the cross-ambiguity function in Section 2.3 that this function enables the task of channel identification from the knowledge of input and output data to the channel. The channel output data are always available since 
this is the received signal. The channel input data are the transmitted symbols which we want to detect and they are not known. The channel rate of variation is always much less than the symbol rate, as shown in Fig. 4-2, and this enables us to use previously detected symbols together with the received signal as the channel input and output.

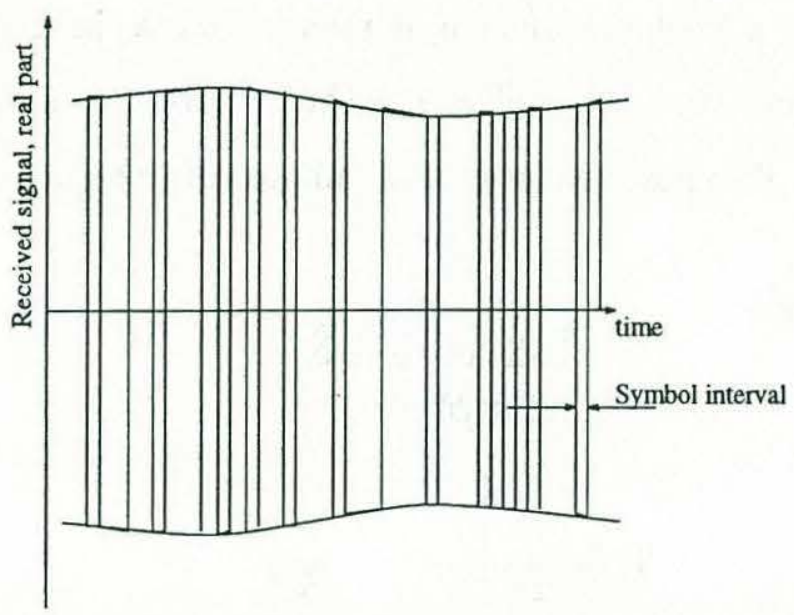

Figure 4-2: The time-variant channel modulates the received signal, but the channel variation is much slower than the symbol rate.

We indicate in Fig. 4-1 that the training sequence is used for two purposes: 1) To compute the cross-ambiguity function that is used for tap allocation in Section 4.3.6 and 2) to obtain initial convergence of the TU-RLS to be presented in Section 4.3.2. In this sense there are two "training modes", but the first involving the tap allocation is the most critical. The training time $T$ below refers to the time of the sequence used to compute the cross-ambiguity function, and the time for TU-RLS convergence is usually a fraction of $T$.

This is the way many practical receivers work [82]: They construct and track the channel response by using the previously detected symbols and the prediction for the current symbol as channel input and the received signal as channel output. The receiver operating in this way yields a delayed channel estimate. The delay is on the order of the number of taps $L$ in the delay direction, and for the receiver and 
channels presented in Chapter $4 L$ is on the order of 10 . A channel is considered rapidly varying if the rate is variation is more than $1 / 100$ of the symbol rate [63], [81]. Therefore the receiver delay is insignificant, and we use the delayed channel estimate as our current estimate. This approach assumes that the decoding is correct or else the channel input is not known. When the decoding is incorrect, the estimation of the channel response will be wrong, and this in turn gives more incorrectly decoded symbols. The phenomenon is known as error propagation and it is present in both the DFE and the TU-RLS receiver to be discussed in Section 4.2 and Section 4.3.

Before we can decode a symbol correctly we must have a reasonable estimate for the channel response, and no previously detected symbols are available from which to construct this. A common way to resolve this is to transmit a sequence of training symbols known to the receiver. In this way the receiver can make use of both channel input and output to construct the channel response estimate. This is called a training sequence, and the necessary length and bandwidth of this sequence depends on the desired resolution and SNR of the channel response estimate. If we want to resolve rays with difference in travel time of $1 \mathrm{msec}$ we need a bandwidth (frequency duration) of $1 \mathrm{kHz}$. If we have rays with different Doppler shifts the dual of this rule is that when we want to resolve Doppler components that are $1 \mathrm{~Hz}$ apart we need a signal time duration of $1 \mathrm{sec}$. Thus resolution in training mode is given by bandwidth and time duration of the training sequence. The finite SNR of the received signal also gives constraints on the training sequence. One interpretation of (2.32) is as the sample cross-covariance function at different Doppler shifts. If the signal model is as in (2.19), the noise term in (2.24) scaled by $1 / T$ approaches the cross-covariance between the transmitted signal and the noise as the integration time $T$ gets large. The signal and noise are assumed to be uncorrelated, therefore the SNR in the cross-ambiguity function increases with the training sequence length. The detailed procedure of allocating taps is presented in Section 4.3.6, and the reader is referred to this chapter for more details. However, the major constraints are the 
ones that we have mentioned here.

We note that there are contradicting concerns between time resolution and good SNR: For good time resolution we want large bandwidths which means more noise in the received signal because the noise is assumed to be white and we are admitting more noise by opening the bandwidth of our receiver filter. There is also a similar tradeoff between the Doppler resolution and the tracking of channel variation: If the Doppler shifts on the rays are time-variant we want to keep the integration time $T$ small enough so that the Doppler is approximately constant over $T$, and this interferes with the requirement to use a signal of long duration in order to resolve Doppler shifts on different rays. In Section 4.3 .6 we present the approach to update the receiver tap locations based on the training sequence and the previous tap locations. When initial convergence of the TU-RLS from the first training sequence from the first packet is achieved, the taps are started with non-zero values estimated by the cross-ambiguity function as described in Section 4.3.7.

The algorithm for tracking used in this work is the RLS and the TU-RLS. The RLS is discussed in [64], and the forgetting factor $\lambda$ determines the tracking bandwidth of the algorithm. A rough rule [64] is that the RLS uses an averaging window of $1 /(1-\lambda)$ samples corresponding to a time window of

$$
\frac{1}{(1-\lambda) f_{s}}
$$

to form the channel response estimate. This is a general and rough rule, and more specific behavior for a specific signal model is derived in the case of RLS in Section 4.2. The motivation for the rough rule is that it enables us to compare the tradeoffs in training mode with the tracking mode where data is being transmitted. In the training mode the averaging window that gives the SNR in the channel response estimate, the Doppler resolution and the tracking bandwidth is given by $T$. In tracking mode we can replace $T$ with (4.16), and then the analysis of the tradeoffs 
is the same as for training mode. Thus in tracking mode a small tracking bandwidth gives high robustness against noise and good Doppler resolution but low capability of tracking changes in the channel such as time-variant Doppler shifts or changing tap allocation vs delay.

A typical communication scenario is that of a packet transmission: A packet of data symbols is prepended with a training sequence and transmitted, and then there is a certain pause before another packet is transmitted. We now discuss the issues involved in determining the ratio of the number of data symbols to training symbols. The length $M$ of the training sequence is governed by the required resolution in delay and Doppler and by the SNR as discussed above. The resolution in delay and Doppler is roughly the reciprocal of the training sequence bandwidth and duration respectively. The noise is present in the cross-ambiguity function that is used for initializing the receiver as discussed in Section 4.3.6. The probability $P_{0}$ for detecting that a signal is present at a given location $\left(l_{0}, k_{0}\right)$ of the cross-ambiguity function is given by (4.178) and shown in Fig. 4-25. In order to maintain a given $P_{0}$ we need a certain $M$. This number is a function of the signal strength present at $\left(l_{0}, k_{0}\right)$. If we require detection of a weak signal with high probability $M$ must be large. Thus the factors that constrain the minimum $M$ are the requirements for Doppler resolution and tap assignment reliability for both delay and Doppler.

The receiver that we develop in Section 4.3 is subject to the WSSUS assumption. As shown in Section 4.3.8, depending on the distribution of $(l, k)$ and $\lambda$, the receivers are stable. This means that for a WSSUS channel with given delay spread, Doppler spread and SNR the receiver reach a stable equilibrium where the probability of decoding error is constant. The SNR is defined as $10 \log \left(\mathcal{E} / \sigma_{w}^{2}\right)$ where $\mathcal{E}$ is the energy per symbol and $\sigma_{w}^{2}$ is the noise variance. For a higher SNR than approximately $6 \mathrm{~dB}$, a lower Doppler spread than $5 \mathrm{~Hz}$ at a symbol rate of $2500 \mathrm{symb} / \mathrm{sec}$ and under the assumption of the channel being WSSUS the receiver approaches steady state with a fixed error rate as shown in Section 4.3.8. When it is re-initialized the receiver 
converges to the same error rate as in steady state. Underwater communication channels are never perfectly WSSUS, and it is partly this fact that in a practical scenario makes it necessary to re-initialize the receiver. There are many ways in which the true channel deviates from a WSSUS channel, and the required rate of re-initialization can only be established when this deviation is characterized. To illustrate this we give an example.

Example: Moving receiver Consider a receiver that moves away from a fixed transmitter at $1 \mathrm{~m} / \mathrm{s}$. The symbol rate is $600 \mathrm{symb} / \mathrm{sec}$ so that the delay resolution is $1.7 \mathrm{msec}$ for a system sampled at the symbol rate. A tap that is initially at the delay $l_{0}$ has moved to $l_{0}+1$ after $2.5 \mathrm{sec}$. If 3 taps were initially allocated and centered at this signal return we need to re-initialize after approximately $5 \mathrm{sec}$ because this is the time it takes for the tap to move outside the receiver allocation range.

Summary The requirement for delay resolution, Doppler resolution and the SNR gives the minimum length of the training sequence from which we compute the crossambiguity function in order to initialize the receiver with a certain reliability. By means of a rough rule for the averaging window in the tracking mode we point out a relationship between the channel estimates in the tracking mode and the training mode. One reason to re-initialize the receiver is that the channel is never perfectly WSSUS, and we give an example of a scenario where this assumption is broken. We re-emphasize that the tracking mode operation requires correctly decoded symbols, and that this is the assumption used in both receivers to be discussed in Section 4.2 and Section 4.3 . 


\subsection{Doppler analysis of a common receiver}

\subsubsection{Preview}

We now consider a modification of a well known and extensively used receiver called a decision feedback equalizer (DFE). The modification is to add a phase locked loop (PLL) that operates jointly with the regular DFE on the data, and we discuss it in particular with respect to the Doppler dimension of the doubly spread underwater communication channel. We find that this receiver is not well suited for Doppler spread signals, and this is an important finding when we consider receivers for doubly spread channels. If this receiver was well suited it would be an obvious candidate because it is a popular receiver in many areas of communication. Since we have a time-variant channel that requires an adaptive receiver with high complexity we consider baseband realizations only. Thus we assume that the complex demodulation and subsampling of the received signal has been carried out, and that the input to the receiver is baseband complex samples at a rate of 2 samples per symbol. The complex demodulation has thus been performed prior to the receiver and is not considered as part of the process here.

It can be argued that a decision feedback equalizer (DFE) should be a good receiver candidate for the underwater communication channel with its often long delay spread. Since the channel is time-variant the DFE needs to be adaptive and the recursive least squares (RLS) algorithm is used to update the taps in order to track the channel. The effect of a Doppler shift is that the signal is phase rotated from symbol to symbol. The receiver needs to apply the opposite phase rotation, and

this is known as tap rotation. To account for common Doppler shifts a phase locked loop (PLL) is used outside the DFE, so that tap rotation due to common Doppler is avoided. We note that the adaptation of the taps as given by the RLS is designed to compensate any signal distortion, including that of Doppler spread. When the PLL is included, in the case of Doppler spread, we have two adaptive algorithms 
compensating the same effect. From an intuitive viewpoint this is unwanted, and we show the consequences. This approach to construct a receiver is taken in [90], and we analyze this receiver with respect to Doppler spread. The signal model of a purely Doppler spread channel (4.20) is used to drive the RLS algorithm in (4.22) and (4.23). We show that the steady state result of this is (4.29). The consequence of this when a DFE is used with the RLS on the same signal is the receiver structure in Fig. 4-5, and we show the maximum Doppler this can accommodate before error propagation occurs. Two versions of the PLL are presented in (4.53) and (4.55), and the interaction between the PLL and the RLS is shown in a set of difference equations in (4.67) and (4.69). We show that there exists marginally stable stationary regions (4.70) and limit cycles (4.76) for his system. Examples of the system behavior is shown in Fig. 4-14-4-16 and the verification on real data is shown in Fig. 4-18 and 419.

\subsubsection{Signal models}

We first review the signal models that are used in the analysis. The transmission channel is a multipath channel, and more specifically it can be modeled as a delay and Doppler spread channel. The presence of Doppler changes, by definition, the delay structure of the channel because the scatterers are moving. We are not considering this effect in our analysis, and thus it is only valid over times that are short enough for this phenomenon not to be significant, see also the example in Section 2.2. The Doppler is different from path to path, and the reason for this may be that the various paths interact with different scatterers at different speeds. If there are $L$ paths with distinct Doppler shifts $\omega_{l}$ impinging on the receiver the received signal is described by

$$
y(n)=\sum_{l=0}^{L-1} y_{l}(n) e^{j \omega_{l} n}
$$


where $y_{l}(n)$ is the signal that is received over ray path $l$. In terms of the transmitted symbols $z(n)$ we write

$$
y_{l}(n)=h_{l}(n) z\left(n-n_{l}\right)
$$

where $h_{l}(n)$ is the time-varying complex attenuation coefficient and $n_{l}$ is the delay of the ray. Note that this allows us to model sparse channels, but this is a minor point in this context since we are now concerned with Doppler spread and not delay spread. Equation (4.17) can be generalized to

$$
y(n)=\sum_{l=0}^{L-1} y_{l}(n) e^{j \phi(n)}
$$

where $\phi(n)$ is the phase shift at time $n$ which can be deterministic or stochastic. Since the objective in this section is analysis with respect to Doppler spread we assume no multipath spread $(L=1)$ and no noise added in the unity gain channel, so the received signal can be written

$$
y(n)=z(n) e^{j \phi(n)}
$$

This is a simple frequency dispersive channel well suited for analyzing Doppler spread, and we use the general $\phi(n)$ in the first part of the analysis and then specialize to $\phi(n)=\omega_{0} n$ when this is appropriate.

Another channel model is given by the impulse response $h(n)$. When we consider constant Doppler shifts it is convenient to separate the common Doppler shift of the channel from its multipath structure. Thus $h(n)$ denotes the channel for zero Doppler, and if there is a Doppler shift $\omega_{0}$ introduced by the channel we receive

$$
y(n)=[h(n) \star z(n)] e^{j \omega_{0} n}
$$


where $\star$ denotes convolution and $z(n)$ is the transmitted sequence which has been whitened. This implies that all paths have the same Doppler, and $h(n)$ is only valid over times short enough to neglect the change is delay structure.

This specifies the different channel models that are used, and they are all timevariant in accordance with the underwater communication channel. Thus the receiver is adaptive in order to track the channel, and we now discuss the adaptive algorithm that is used in the receiver.

Summary We represent the received signal as a sum of differently attenuated and Doppler spread contributions arriving over a number of ray paths. The objective is analysis with respect to Doppler spread, and thus the model in (4.20), corresponding to a single Doppler spread ray, is especially useful.

\subsubsection{RLS algorithm}

The adaptive algorithm that is used in the DFE is the RLS algorithm, and this is chosen over the LMS because of its faster convergence. It is known that the convergence speed of the LMS depends on the spread in the eigenvalues of the channel covariance matrix. A large spread yields slow convergence whereas the RLS convergence speed is independent of this. The RLS is also related to the Kalman filter and the TU-RLS presented in Section 4.3. The RLS algorithm is derived and discussed in e.g. [45], [64], but the objective here is to derive steady state expressions for the algorithm when it is driven with a Doppler spread signal. This information is used to interpret how the steady state DFE will process the received signal. The result and the assumptions we make to obtain the steady state in (4.29) and (4.35) are in accordance with [65], [31]. We now want to define and analyze the steady state characteristics of the RLS algorithm. The RLS equations are [45]

$$
\hat{z}(n)=\mathbf{a}^{H}(n) \mathbf{c}(n)
$$




$$
\begin{gathered}
e(n)=z(n)-\hat{z}(n) \\
\mathbf{a}(n+1)=\mathbf{a}(n)+\mathbf{k}(n) e^{*}(n) \\
\mathbf{k}(n)=\frac{\frac{1}{\lambda} P(n-1) \mathbf{c}(n)}{1+\frac{1}{\lambda} \mathbf{c}^{H}(n) P(n-1) \mathbf{c}(n)} \\
P(n)=\frac{1}{\lambda} P(n-1)-\frac{1}{\lambda} \mathbf{k}(n) \mathbf{c}^{H}(n) P(n-1)
\end{gathered}
$$

where $\mathrm{k}(n)$ is the $L \times 1$ gain vector, $e(n)$ is the scalar error and $P(n)$ is an $L \times L$ matrix. The symbol $\mathbf{c}^{H}$ denotes complex conjugate transpose of $\mathbf{c}$ and $\lambda$ denotes the forgetting factor. The data is contained in the vector $\mathbf{c}(n)$ :

$$
\mathbf{c}(n)=[y(n) \cdots y(n-L+1)]^{T} .
$$

$\mathbf{a}(n)$ is the $L \times 1$ parameter vector that is adapted to minimize the error, $\hat{z}(n)$ is the signal estimate and $z(n)$ is the desired signal. $L$ is the system order and $y(n)$ is the observed data.

Note The first and last equations of (4.22) are usually written $\mathbf{a}(n)=\mathbf{a}(n-$ 1) $+\mathbf{k}(n) e^{*}(n), \hat{z}(n)=\mathbf{a}^{H}(n-1) \mathbf{c}(n)$ in the traditional RLS context. We show in Section 4.3.2 that the RLS may be interpreted as a Kalman filter where the system matrix $A=I$ and $\lambda=1$. Thus the update step of the RLS in the Kalman filtering context is $\mathbf{a}(n+1)=I \mathbf{a}(n)$ which is equivalent to $\mathbf{a}(n)=I \mathbf{a}(n-1)$. In order to unify the treatment we chose the form in (4.22).

The recursion for $P(n)$ can be written by means of (4.23)

$$
\begin{aligned}
\lambda^{2} P(n) & +\lambda P(n)\left(\mathbf{c}^{H}(n) P(n-1) \mathbf{c}(n)\right)=\lambda P(n-1) \\
+ & P(n-1) \mathbf{c}^{H}(n) P(n-1) \mathbf{c}(n)-P(n-1) \mathbf{c}(n) \mathbf{c}^{H}(n) P(n-1)
\end{aligned}
$$

where $P(0)$ is chosen to be a large number times the identity matrix. The reason 
for a large number is motivated by (4.23), because it will start the gain vector $\mathrm{k}(0)$ large so that initial convergence is fast.

The equation (4.25) is a matrix difference equation with random coefficients because the data $\mathbf{c}(n)$ is random. We now assume that there exists a matrix $\mathrm{P}$ that is independent of time and that is the solution to the mean of the equation above. Thus we insert $P(n-1)=P(n)=P$ and take expectations in (4.25). It is not obvious that we are justified in these assumptions, but if a solution exists under these assumptions it may be interpreted as a steady state value. A similar approach is taken in [31], [65], and the results in these references are in accordance with this work. In order to arrive at (4.26) these references neglect certain statistical dependencies between variables, whereas we avoid this by assuming that $P$ is a deterministic matrix. We obtain after some straightforward manipulations of (4.25)

$$
P\left(\lambda^{2} I+\lambda E\left[\mathbf{c}^{H}(n) P \mathbf{c}(n)\right] I\right)=P\left(\lambda I+E\left[\mathbf{c}^{H}(n) P \mathbf{c}(n)\right] I-E\left[\mathbf{c}(n) \mathbf{c}^{H}(n)\right] P\right) .
$$

$\mathrm{P}$ is assumed to be invertible, so we can multiply the equation by $P^{-1}$ and rearrange to get

$$
\begin{aligned}
E\left[\mathbf{c}(n) \mathbf{c}^{H}(n)\right] P & =(1-\lambda) E\left[\mathbf{c}^{H}(n) P \mathbf{c}(n)\right] I-\lambda(\lambda-1) I \\
E\left[\mathbf{c}(n) \mathbf{c}^{H}(n)\right] P & =\left[(1-\lambda) E\left[\mathbf{c}^{H}(n) P \mathbf{c}(n)\right]-\lambda(\lambda-1)\right] I \\
& =\chi I .
\end{aligned}
$$

The right hand side of (4.27) is a scalar $\chi$, as defined by (4.28), times the identity matrix, so we get

$$
P=\chi E\left[\mathbf{c}(n) \mathbf{c}^{H}(n)\right]^{-1} .
$$

We note that the expected value of the outer product in (4.29) must be full rank in order for $P$ to exist. The steady state value of $\mathrm{P}(\mathrm{n})$, which has been shown [65] to be close to $P$ in a mean square sense, is a scalar times the inverse of the input 
signal covariance. The following derivation shows that $\chi$ is not a function of $P$, so $P$ is proportional to the inverse of the input signal covariance. By inserting (4.29) in (4.27) we get

$$
E\left[\mathbf{c}(n) \mathbf{c}^{H}(n)\right] P=(1-\lambda) E\left[\mathbf{c}^{H}(n) \chi E\left[\mathbf{c}(n) \mathbf{c}^{H}(n)\right]^{-1} \mathbf{c}(n)\right] I-\lambda(\lambda-1) I
$$

and this is solved to get an expression for the scaling factor $\chi$ :

$$
\chi=\frac{\lambda(1-\lambda)}{1-(1-\lambda) E\left[\mathbf{c}^{H}(n) E\left[\mathbf{c}(n) \mathbf{c}^{H}(n)\right]^{-1} \mathbf{c}(n)\right]}
$$

The expectation expression in the denominator equals $L$. This is seen by identifying the quadratic form inside the outer expectation as

$$
\mathbf{c}^{H}(n) E\left[\mathbf{c}(n) \mathbf{c}^{H}(n)\right]^{-1} \mathbf{c}(n)=\operatorname{tr}\left[\mathbf{c}^{H}(n) E\left[\mathbf{c}(n) \mathbf{c}^{H}(n)\right]^{-1} \mathbf{c}(n)\right] .
$$

Using $\operatorname{tr}(B A)=\operatorname{tr}(A B)$ with $B=\mathbf{c}^{H}(n)$ and $A=E\left[\mathbf{c}(n) \mathbf{c}^{H}(n)\right]^{-1} \mathbf{c}(n)$ we get

$$
\operatorname{tr}\left[\mathbf{c}^{H}(n) E\left[\mathbf{c}(n) \mathbf{c}^{H}(n)\right]^{-1} \mathbf{c}(n)\right]=\operatorname{tr}\left[E\left[\mathbf{c}(n) \mathbf{c}^{H}(n)\right]^{-1} \mathbf{c}(n) \mathbf{c}^{H}(n)\right] .
$$

Now applying the outer expectation gives

$$
\begin{aligned}
& E\left[\operatorname{tr}\left[E\left[\mathbf{c}(n) \mathbf{c}^{H}(n)\right]^{-1} \mathbf{c}(n) \mathbf{c}^{H}(n)\right]\right]= \\
& \operatorname{tr}\left[E\left[\mathbf{c}(n) \mathbf{c}^{H}(n)\right]^{-1} E\left[\mathbf{c}(n) \mathbf{c}^{H}(n)\right]\right]=L .
\end{aligned}
$$

Thus the scaling factor is given by

$$
\chi=\frac{\lambda(1-\lambda)}{1-(1-\lambda) L} .
$$

This expression is shown in Fig. 4-3 where $\chi$ is plotted vs $\lambda$ with $L$ as parameter. The expression (4.35), and the derivation in general, is only valid for $L \ll 1 /(1-\lambda)$. 


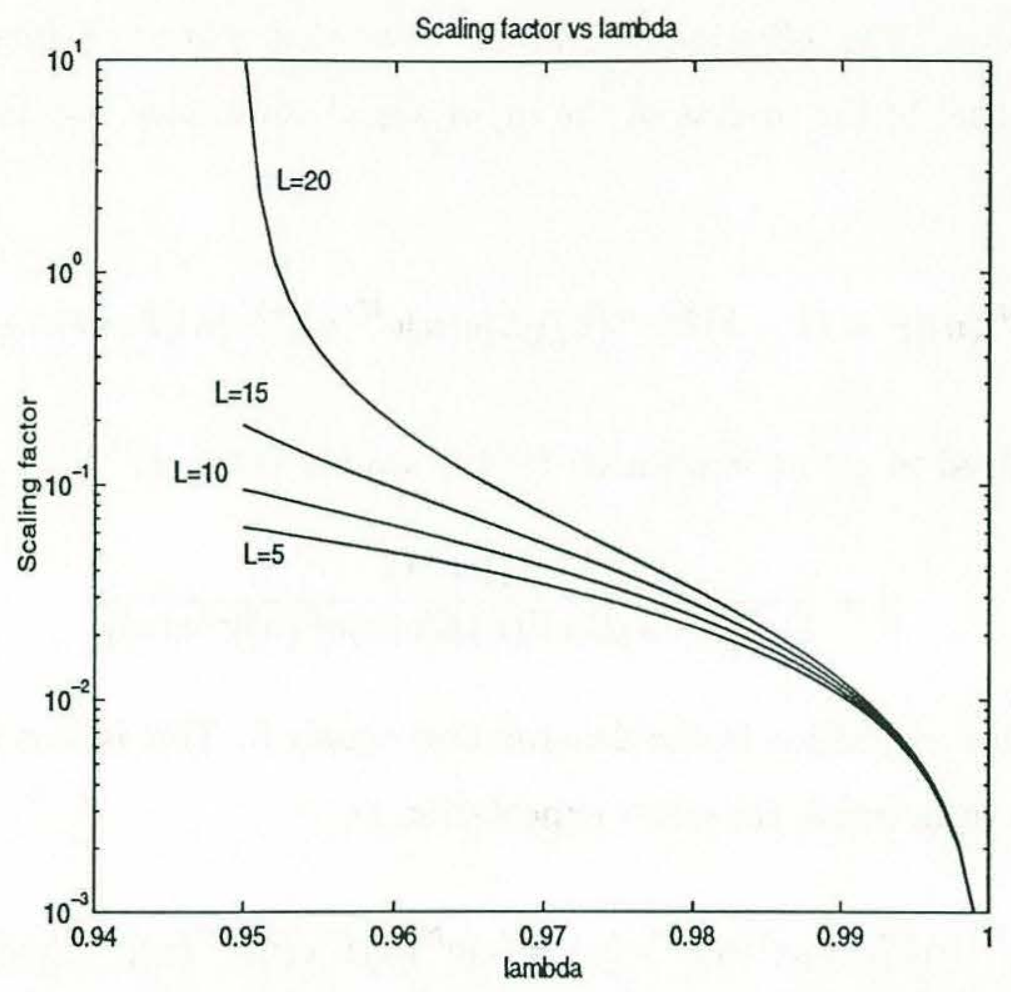

Figure 4-3: The scaling factor of the steady state solution vs $\lambda$ with the number of taps $L$ as parameter.

The reason for this is that $P$ becomes close to rank deficient, and this is shown by the discussion of (4.37) below. Some additional insight can be obtained by examining the result if this assumption is violated. Therefore let us now allow $L$ to be arbitrary, and then we have that

$$
\chi \rightarrow \infty \text { as } L \rightarrow \frac{1}{1-\lambda}
$$

The reason for this behavior of $\chi$ is the following: The matrix $P$ is computed from a number of the most recent data vectors. Due to the exponential windowing in RLS, the number of data vectors used, or the window length, is roughly $1 /(1-\lambda)$.

$$
P \sim \sum_{k=0}^{1 /(1-\lambda)} \mathbf{c}(n+k) \mathbf{c}^{H}(n+k)
$$

where the sign $\sim$ means "proportional to". The outer products $\mathbf{c}(n+k) \mathbf{c}^{H}(n+k)$ 
are rank one, so the maximum rank of the right hand side sum in (4.37), which is approximately $P$, is $1 /(1-\lambda)$. Thus, as $L$ approaches $1 /(1-\lambda), P$ becomes very close to singular. This is reflected in $\chi$ approaching infinity for this value of $L$.

The solution we get from the employed assumptions is a positive definite matrix, and the expressions correspond well with numerical values for $P$ and $\chi$ obtained by simulation of the difference equation (4.23) for $P$. This is performed as part of the verification of (4.29) where Gaussian noise is used to model the data in $\mathbf{c}(n)$, and both white and nonwhite noise give answers predicted by (4.29) and (4.35).

Summary We analyze the RLS in presence of a Doppler spread signal, and the difference equation (4.23) that determines the gain is found to have a steady state solution given by (4.29) and (4.35). This solution suggests that the steady state of the matrix $P$, determining the gain, is given by the exponential weighting $\lambda$ and the input signal covariance matrix, and this is verified by simulations of (4.23).

\subsubsection{Receiver structure}

We now consider the DFE for acoustic underwater communication [90], which is shown in Fig. 4-4, and the modulation is assumed to be QPSK. The various parts of the receiver has different physical interpretations which we now list.

- The FIR filter $a_{1}(n)$ is the feedforward part of the DFE, and it is used to combine signal energy from different time lags.

- The FIR filter $a_{2}(n)$ is the feedback part which subtracts out ISI generated by previous symbols. This filter is driven by the decoded signal $\tilde{z}(n)$ which is identical to $z(n)$ if the decoding is correct.

- The sequences $p_{1}(n)$ and $p_{2}(n)$ are the outputs of the feedforward and feedback filters. 
- The quantizer $Q()$ takes the soft signal estimate $\hat{z}(n)$ into one of four legal symbols which in the case of QPSK are given by (2.16).

The coefficients $\mathbf{a}$ of (4.22) are split into two parts $\mathbf{a}=\left[\begin{array}{ll}\mathbf{a}_{1} & \mathbf{a}_{2}\end{array}\right]$ in order to obtain this interpretation.

Consider the simple channel, dispersive in frequency, described by (4.20). We want to analyze how the receiver handles Doppler, so this channel model is sufficient for the issue addressed. If we transmit a whitened sequence $z(n)$ and assume that the channel gain is unity we receive

$$
y(n)=z(n) e^{j \phi(n)} .
$$

The assumptions in (4.38) are a Doppler spread signal arriving over a single ray

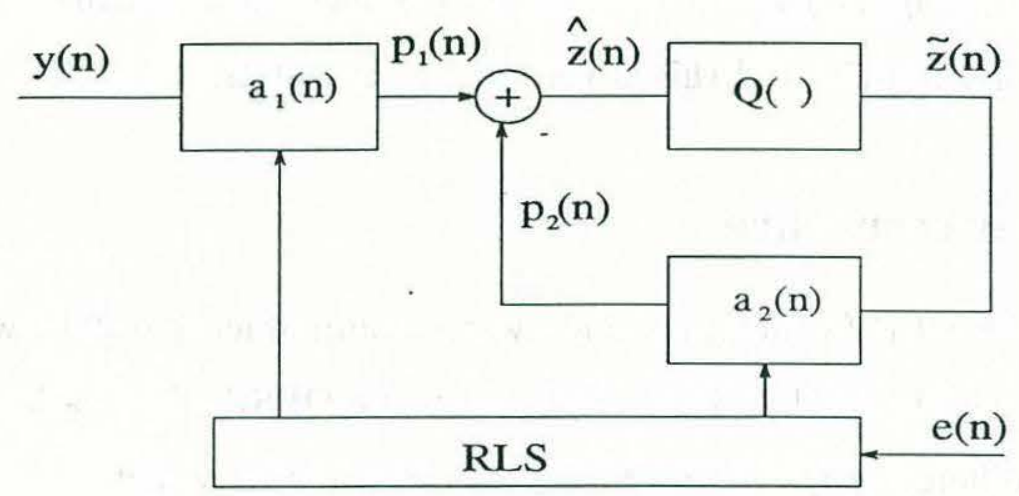

Figure 4-4: A single channel receiver, all parameters adapted with RLS.

path with no amplitude fluctuation, and the noise is assumed to be zero since this is not a major point for the analysis with respect to the Doppler spread. The phase $\phi(n)$ is a correlated sequence that determines the Doppler spread. The maximum rate of variation is one of the concerns in this analysis, and we find an expression for this rate that is valid under specific assumptions.

The channel model (4.38) gives no ISI caused by multipath in the received signal since there is no delay spread in the channel. Thus it is plausible that the steady 
state receiver feedback part converges to zero because the purpose of this part of the receiver is to remove ISI from previously detected symbols. By the same argument the feedforward section will have a single tap, since the purpose of this filter is to combine signal energy at different lags and the symbols in our case are not spread in delay. Thus we get the receiver in Fig. 4-5. The structure of the steady state DFE depends in general on the bandwidth of the process $\phi(n)$. We assume that it is a correlated process that varies slowly compared to the symbol rate. The steady state configuration is verified by running the DFE on both real data with no ISI and simulated data with the channel model (4.38). We note that this receiver implies the special case of the scalar RLS equation which means that $\mathbf{a}(n), \mathrm{k}(n), P(n)$ and $\mathbf{c}(n)$ are all scalars.

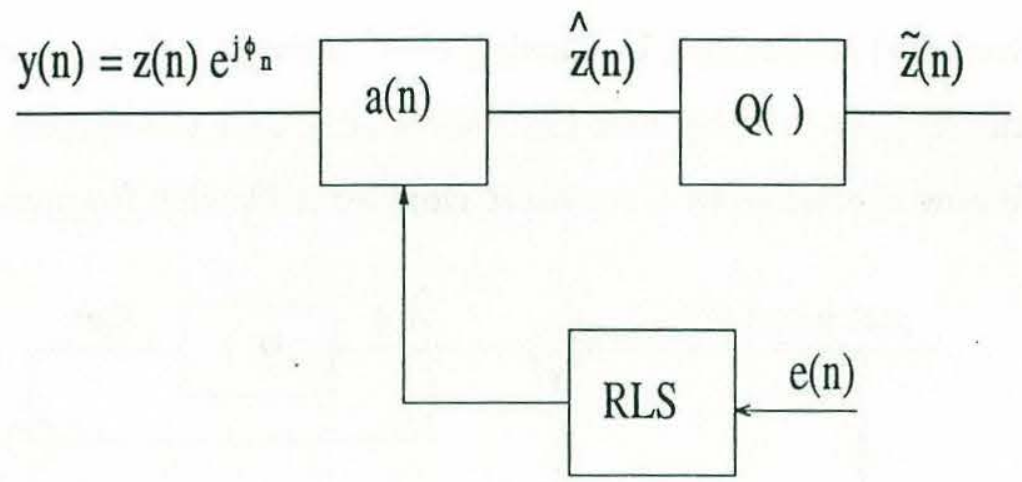

Figure 4-5: The matched receiver that must handle the Doppler shift in the input signal. With no ISI the feedback part of the DFE is absent.

For the very simple system considered here the solution $P$ to (4.23) that is independent of time is given by inserting $P(n)=P(n-1)=P$ (by definition of steady state) into this equation. This yields

$$
P=1-\lambda
$$

where we have used that $z z^{*}=1$ for QPSK modulation and that there is no noise in our channel model (4.38). This is in correspondence with the result obtained from 
the more general (4.29). We find the steady state gain by using (4.23) and (4.39) which yields

$$
k(n)=(1-\lambda) y(n) .
$$

We assume that the output of the decision device is the correct data, $\tilde{z}(n)=z(n)$. This implies that the receiver is either in a training mode where correct decisions are supplied externally or that the receiver is decoding correctly. We also assume that the receiver has been able to converge to steady state, so that (4.39) and (4.40) are valid. Thus we get from (4.22) and (4.23)

$$
\begin{aligned}
a(n+1) & =a(n)+(1-\lambda) z(n) e^{j \phi(n)}\left(z(n)-a^{*}(n) z(n) e^{j \phi(n)}\right)^{*} \\
& =\lambda a(n)+(1-\lambda) e^{j \phi(n)} .
\end{aligned}
$$

We can see that $a(n)$ is obtained by passing $e^{j \phi(n)}$ through a first order IIR low pass filter where the dc gain is unity and the pole is at $\lambda$, and the system is depicted in Fig. 4-6. We now specialize to a constant Doppler shift with frequency $\omega_{0}$,

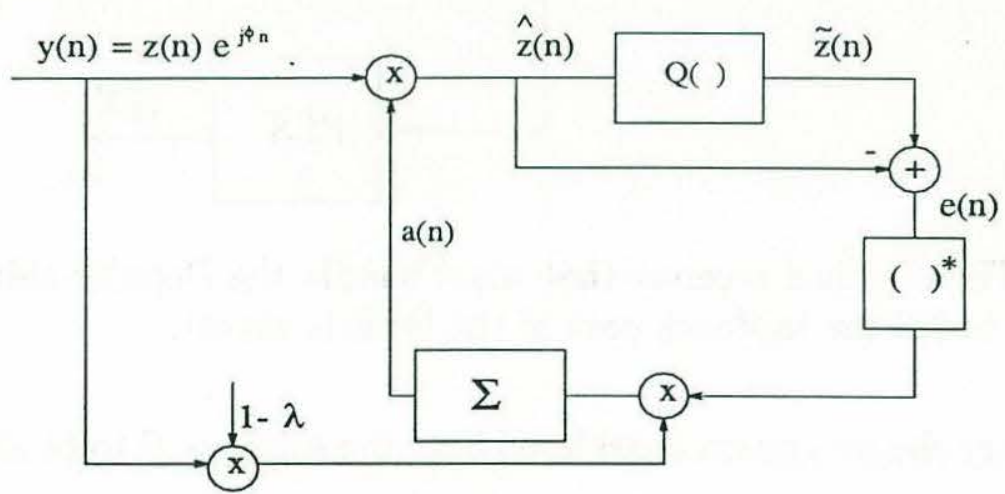

Figure 4-6: The equivalent RLS phase locked loop. $\Sigma$ is a summer (discrete integrator).

$$
\phi(n)=\omega_{0} n
$$


so that now the signal model is

$$
y(n)=z(n) e^{j \omega_{0} n} .
$$

In the frequency domain we have from (4.41) and (4.42)

$$
A\left(e^{j \omega}\right)=\frac{1-\lambda}{1-\lambda e^{-j \omega}} e^{-j \omega} \delta\left(\omega-\omega_{0}\right)=H\left(e^{j \omega}\right) \delta\left(\omega-\omega_{0}\right)
$$

For large $n$ the filter tap $a(n)$ is a complex exponential with amplitude $\left|H\left(e^{j \omega_{0}}\right)\right|$ and angle $\angle H\left(e^{j \omega_{0}}\right)$. This is valid as long as the decoding is correct, i.e., for QPSK as long as

$$
|\angle \hat{z}(n)-\angle z(n)|<\frac{\pi}{4}
$$

When this condition is violated the decisions will be incorrect. This implies that (4.41) and (4.44) are violated, and the structure in Fig. 4-6 is invalid. The filter $H\left(e^{j \omega}\right)$ determining the filter $\operatorname{tap} a(n)$ is shown in Fig. 4-7. When the RLS is
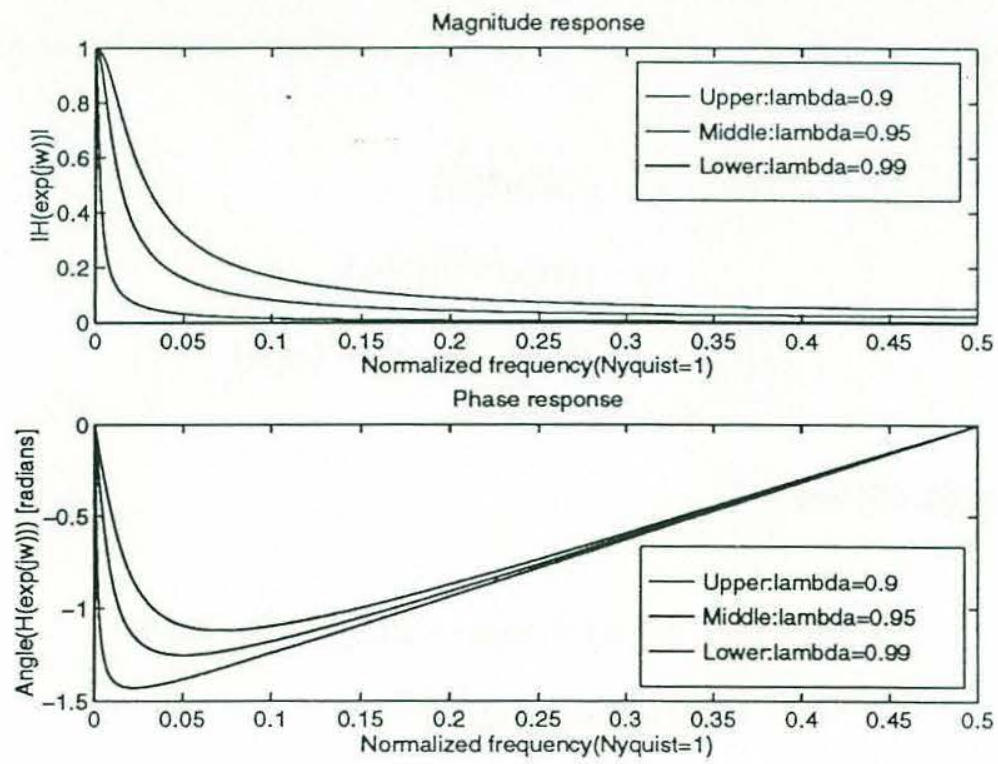

Figure 4-7: Magnitude (top) and phase of the filter determining the filter tap $a(n)$ when the RLS algorithm is tracking a constant Doppler. 
driven by a signal as in (4.20) the steady state filter tap $a(n)$ tracks the phase of the input signal. This is the same function as a PLL. This RLS-PLL, not to be confused with the regular PLL discussed later, is tracking when (4.45) is satisfied. Ideally the incoming phase $\omega_{0} n$ should be compensated by $\angle H\left(e^{j \omega_{0}}\right)$, and we have that $a(n)$ is generated by passing $e^{j \omega_{0} n}$ through the filter $H\left(e^{j \omega}\right)$ as shown in Fig. 4-8. Thus in

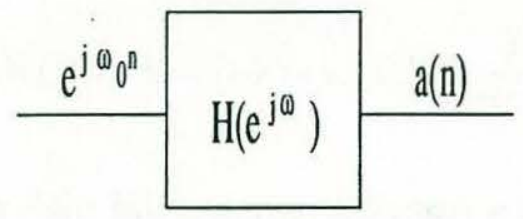

Figure 4-8: The filter tap is generated from a low pass filter driven by a complex sinusoid that is the Doppler shift.

steady state we have

$$
\begin{aligned}
a(n) & =\left|H\left(e^{j \omega_{0}}\right)\right| e^{L H\left(e^{j \omega_{0}}\right)} e^{j \omega_{0} n} \\
\angle a(n) & =\angle H\left(e^{j \omega_{0}}\right)+\omega_{0} n .
\end{aligned}
$$

When the incoming sample is $y(n)=z(n) e^{j \omega_{0} n}(4.22)$ in steady state yields

$$
\begin{aligned}
\hat{z}(n) & =y(n) a^{*}(n) \\
& =z(n) e^{j \omega_{0} n} a^{*}(n) \\
\angle \hat{z}(n) & =\angle z(n)+\omega_{0} n-\angle a(n)
\end{aligned}
$$

and by inserting (4.46) we get

$$
\begin{aligned}
L \hat{z}(n) & =\angle z(n)+\omega_{0} n-\angle H\left(e^{j \omega_{0}}\right)-\omega_{0} n \\
& =\angle z(n)+\angle H\left(e^{j \omega_{0}}\right) .
\end{aligned}
$$

Thus in order for the phase estimate $\angle \hat{z}(n)$ to equal the true value $\angle z(n)$ the angle of $\mathrm{H}$ should be zero. If it is more than $\pi / 4$ the correct decoding assumption (4.45) 
is violated, and the RLS-PLL goes out of lock. This is satisfied when

$$
\begin{aligned}
\angle H\left(e^{-j \omega_{0}}\right) & =\tan ^{-1} \frac{\lambda \sin \left(\omega_{0}\right)}{1-\lambda \cos \left(\omega_{0}\right)}=\frac{\pi}{4} \\
\omega_{0} & =\cos ^{-1}\left[\frac{1}{2 \lambda}+\sqrt{\frac{1}{2}-\frac{1}{4 \lambda^{2}}}\right] .
\end{aligned}
$$

Fig. 4-9 shows the maximum Doppler that can be handled by the RLS-PLL given by (4.49). For a representative $\lambda$ of 0.98 we get $\omega_{0}=0.0206$ corresponding to a

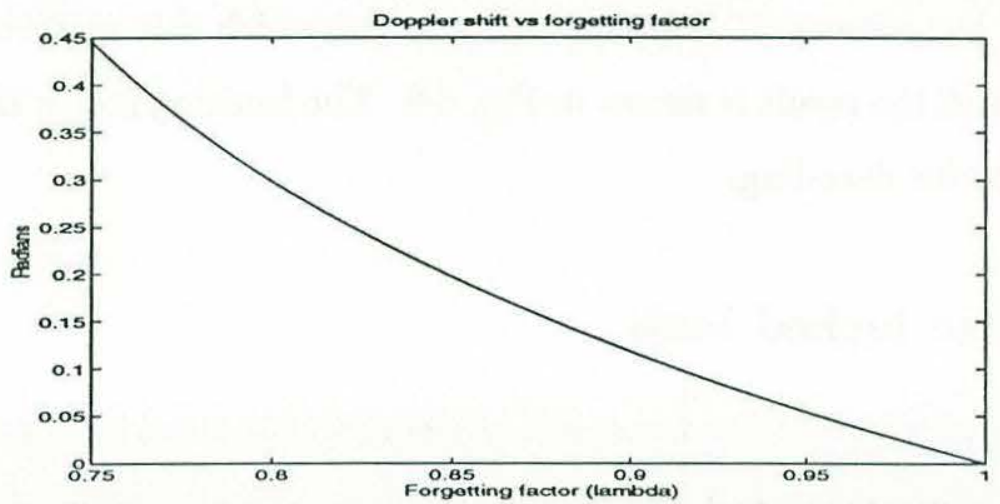

Figure 4-9: The maximum Doppler shift that can be corrected by the RLS-PLL is a function of the forgetting factor $\lambda$.

Doppler shift of a fraction of a percent of the symbol rate. This corresponds to a Doppler shift of $\nu_{0}=2 \mathrm{~Hz}$ at a symbol rate of $600 \mathrm{symb} / \mathrm{sec}$. Thus the RLS cannot handle significant Doppler shifts. The filter $a(n)$ is usually started with $a(0)=0$, and when $z(n) e^{j \omega_{0} n}$ arrives the phase of $A\left(e^{j \omega_{0}}\right)$ starts at zero and builds up towards its final value. By summing up (4.41) we get:

$$
a(n+1)=(1-\lambda) \frac{1-\left(\lambda e^{j \omega_{0}}\right)^{n}}{1-\lambda e^{j \omega_{0}}} e^{j \omega_{0} n} .
$$


The speed with which the phase $\angle a(n)$ approaches its asymptote is given by the time constant $\lambda$ of the filter which is the same as the RLS forgetting factor. It is given by

$$
\angle a(n+1)=\tan ^{-1} \frac{\dot{\lambda}^{n} \sin \left(\omega_{0} n\right)}{1-\lambda^{n} \cos \left(\omega_{0} n\right)}-\tan ^{-1} \frac{\lambda \sin \left(\omega_{0} n\right)}{1-\lambda \cos \left(\omega_{0} n\right)}+\omega_{0} n
$$

Summary We present a DFE adapted with RLS in Fig. 4-4, and we show that the steady state of this receiver reduces to the configuration in Fig. 4-6 when driven with a purely Doppler spread signal from (4.20). The steady state solution for the RLS is used to find the amount of Doppler spread under which this receiver can operate satisfactorily, and the result is shown in Fig. 4-9. The limiting factor is the feedback of symbols used for decoding.

\subsubsection{Phase locked loop}

There are several ways for the transmitted signal to be distorted by Doppler, and some of them were mentioned earlier in this work. The analysis in Section 4.2.3 shows that the RLS algorithm is very sensitive to Doppler, and not very capable of handling significant Doppler shifts. A way to overcome this is to use a phase locked loop to remove the Doppler shift from the signal before it enters the rest of the receiver. In this section we discuss some of the features of the phase locked loop (PLL). The PLL is embedded in the DFE, therefore some features that are from this part of the receiver are also discussed in this section.

A classical PLL is built up of three components as shown in Fig. 4-10, and this PLL works on complex signals in discrete time. The PLL is derived from an extended Kalman filter [105]. Consider the signal given in (4.21), and remember that the input signal $y(n)$ is a baseband signal sampled at 2 samples per symbol. Thus the purpose of the PLL is not carrier recovery but tracking and elimination of a Doppler shift present in the demodulated data. If an $\mathrm{m}$-sequence is used as the transmit signal and the modulation used is QPSK this signal can not be used to drive a PLL 


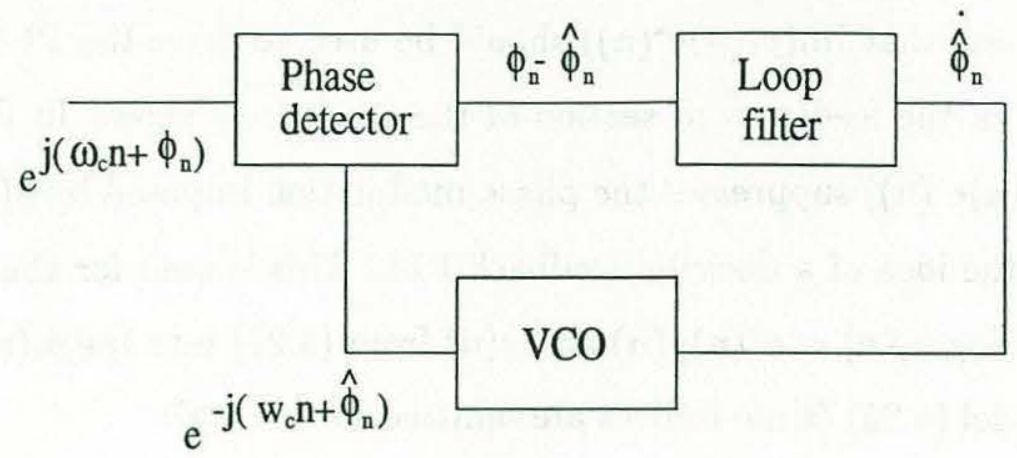

Figure 4-10: Building blocks of a PLL.

directly. The input signal spectrum is not centered in a narrow band around any frequency $\omega_{c}$, and this violates a basic assumption of the PLL in Fig. 4-10. Thus the effect of the transmitted signal must be taken out before using a PLL to track $\omega_{0}$. Ideally the PLL should be driven with $e^{j \omega_{0} n}$. If we modulate the incoming signal with a signal containing $z^{*}(n)$ this effect is achieved. This modulation is used in a decision feedback PLL [80], [71], and the PLL characteristics are discussed in these references.

Much of the literature on PLL's assumes a real input signal whereas we have a complex input signal, and this implies that the phase detector (PD) must be modified. Its function is to extract the phase of the incoming signal and compare it to the estimated phase as shown in Fig. 4-10. There are two ways of extracting the phase difference (i.e., two phase detectors) that are fairly common, and in current use in the receiver described in Section 4.2.6. They are hereafter called the imaginary part PD and the angle PD.

\section{Imaginary part PD}

The idea of jointly adapting the PLL and the DFE in the manner we discuss is reported in [90]. In this work an approach is taken where $\phi(n)=\omega_{0} n$ and the filter taps $\mathbf{a}(n)$ are treated as random variables. The cost criterion is the mean square 
error (MSE) of the decoded data sequence. It is interesting that joint optimization of a $(n)$ and $\phi(n)$ suggests that $\operatorname{Im}\left(p_{1}(n) e^{*}(n)\right)$ should be used to drive the PLL where $p_{1}(n)$ is the output of the feedforward section of the receiver as shown in Fig. 4-4. The product $\operatorname{Im}\left(p_{1}(n) e^{*}(n)\right)$ suppresses the phase modulation imposed by $z(n)$, and this is in line with the idea of a decision feedback PLL. This is seen for the case of scalar $a(n)$ by inserting $p_{1}(n)=a^{*}(n) y(n)$ and $e(n)$ from (4.22) into $\operatorname{Im}\left(p_{1}(n) e^{*}(n)\right)$ using the signal model (4.20) (time indices are omitted for clarity):

$$
\operatorname{Im}\left(p_{1} e^{*}\right)=\operatorname{Im}\left(a^{*} z e^{j \phi}\left(z^{*}-\hat{z}^{*}\right)\right) .
$$

Since we are using QPSK modulation we have that $z z^{*}=1$ for the receiver used in [90]. With our signal model the steady state receiver is as in Fig. 4-5. This gives

$$
\begin{aligned}
\operatorname{Im}\left(p_{1} e^{*}\right) & =\operatorname{Im}\left(a^{*} z e^{j \phi}\left(z^{*}-a z^{*} e^{-j \phi}\right)\right) \\
& =\operatorname{Im}\left(a^{*}\left(e^{j \phi}-a\right)\right) \\
& =\operatorname{Im}\left(a^{*} e^{j \phi}\right) \\
& =|a| \sin \left(\angle\left[a^{*} e^{j \phi}\right]\right)
\end{aligned}
$$

and thus $\operatorname{Im}\left(p_{1} e^{*}\right)$ contains no phase modulation from $z$. We can also use standard identities and write

$$
\begin{aligned}
p_{1} e^{*} & =\left|p_{1} e^{*}\right|\left(\cos \left(\angle\left[p e^{*}\right]\right)+j \sin \left(\angle\left[p_{1} e^{*}\right]\right)\right) \\
\operatorname{Im}\left(p_{1} e^{*}\right) & =\left|p_{1} e^{*}\right| \sin \left(\angle\left[p_{1} e^{*}\right]\right) .
\end{aligned}
$$

By using the usual small angle approximation $\sin (\phi) \approx \phi$ on $\angle\left[p_{1} e^{*}\right]$ this PLL follows the analysis in regular texts on PLL's [82]. 


\section{Angle PD}

The PD is sometimes modified, and the modification reported in [53] is in current use for a receiver similar to the one we discuss. In an attempt to mimic the function of the PLL in Fig. 4-10 the phase detector is taken as a multiplier followed by phase angle extraction (time indices are dropped for clarity):

$$
\angle\left[p_{1} e^{*}\right]=\angle x_{e}=\tan ^{-1} \frac{\operatorname{Im}\left(x_{e}\right)}{R e\left(x_{e}\right)} .
$$

where we define $x_{e}=p_{1} e^{*}$ for brevity. The idea is that the PD ideally should deliver the instantaneous phase difference between the feedback and input signal as shown in Fig. 4-11. Since we argue that $p_{1} e^{*}$ should be used to drive the PLL (4.55) follows naturally. The PD of Fig. 4-10 corresponds to the multiplier and angle operator of Fig. 4-11, the filter $g(n)$ of Fig. 4-11 is the loop filter in Fig. 4-10 and the sum and exponential operator of Fig. 4-11 are the VCO of Fig. 4-10. The loop filter has the same function and form as in Fig. 4-10. It changes the control characteristics of the feedback loop and modify important system parameters such as the sensitivity function of the closed loop system [5]. The voltage controlled oscillator (VCO) is an integrator followed by a complex sinusoidal generator. The resulting PLL should

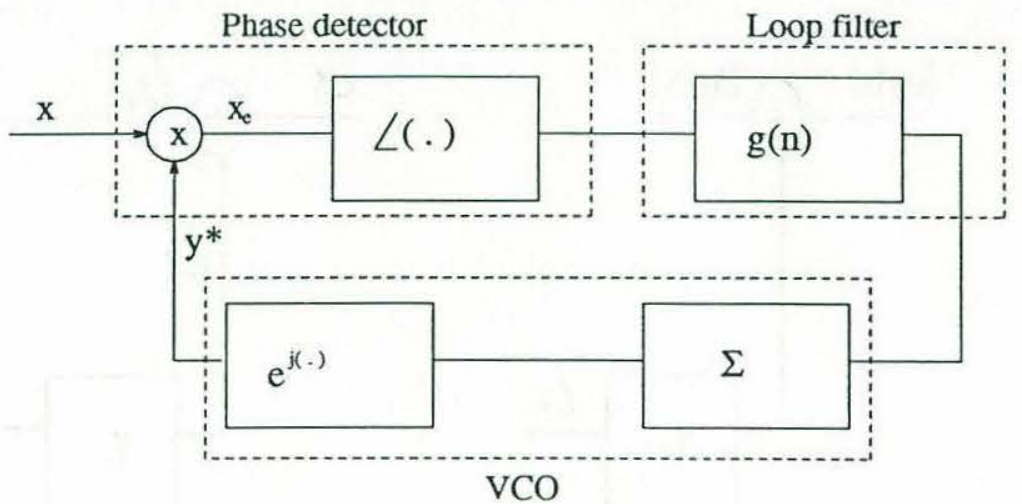

Figure 4-11: The structure of the digital PLL, working on complex signals. $\Sigma$ is a summer (discrete integrator).

be designed for tracking the angle of the input signal. It is nonlinear, and it can 
be interpreted as two linear subsystems that are coupled with a nonlinear equation. Fig. 4-11 gives us

$$
x_{e}=x y^{*}=x e^{-j\left(\gamma \star L x_{e}\right)}
$$

where $\gamma(n)$ is the composite effect of $g(n)$ in Fig. 4-11 and the integrator, and it is called the loop transfer function. The symbol $\angle$ means the physical, unwrapped, angle and not only the principal angle that is between $\pm \pi$. Taking the logarithm of the complex quantity $x_{e}$ we get

$$
\log \left|x_{e}\right|+j \angle x_{e}=\log |x|+j \angle x-j \gamma \star \angle x_{e} .
$$

The real and imaginary part of this equation must be separately satisfied, and thus we have the structure in Fig. 4-12 which can be written as

$$
\begin{aligned}
\angle x_{e} & =\angle x-\gamma_{r} \star \angle x_{e} \\
\log \left|x_{e}\right| & =\log |x|+\gamma_{i} \star \angle x_{e} .
\end{aligned}
$$

The angle dynamics are given by the real part $\gamma_{r}$ of the loop filter and VCO (loop

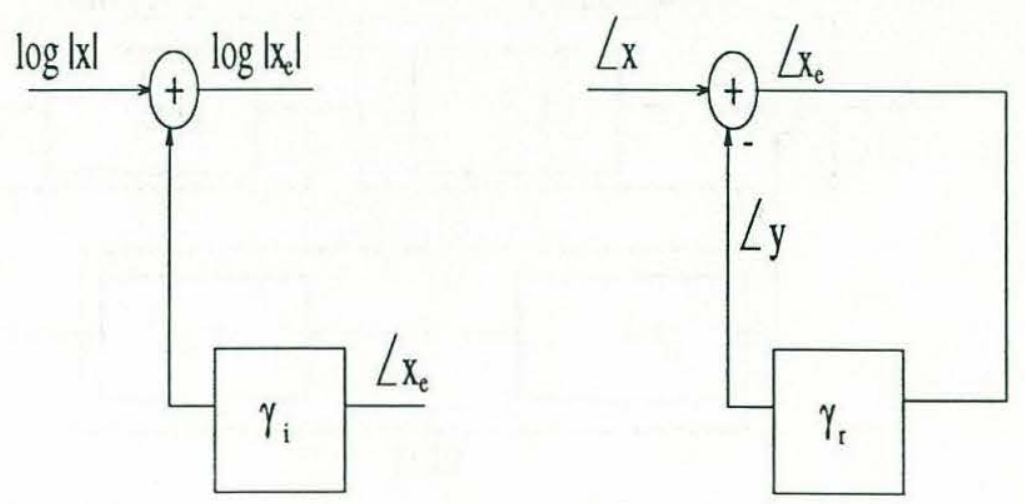

Figure 4-12: The phase and modulus of the signal going into the PLL is found by considering these structures. 
transfer function). If the imaginary part $\gamma_{i}$ of the loop transfer function is zero, the absolute value is unaffected. This enables us to apply control theory in the design and analysis of the PLL angle tracking performance. The most important assumption for this analysis to be true is that the angle error $\angle x_{e}$ is small. If the condition

$$
\left|\angle x_{e}\right|<\pi
$$

is violated we get cycle skipping, because the complex logarithm in (4.58) delivers only the principal angle. When the PLL is in normal operation (4.59) is satisfied and when the PLL is out of lock the condition is violated.

Summary We present the general PLL in Fig. 4-10, and discuss two realizations with different phase detectors called the imaginary part PD (4.52) and angle PD (4.55). The imaginary part PD corresponds to the classical PLL, using the small angle approximation in (4.54). The angle PD is motivated from heuristic arguments mentioned in the discussion of (4.55) and from its practical usefulness.

\subsubsection{Interaction between phase locked loop and filter adap- tation}

Preview We now combine the results on the RLS and the PLL presented in Section 4.2.3 and Section 4.2.5 to characterize the composite system. We find that in the two cases of imaginary part PD and angle PD the system is governed by the nonlinear, coupled sets of difference equations (4.67) and (4.69). We find the stationary regions (4.70) and limit cycles (4.76) to these difference equations. Then we determine the stability of the stationary regions, and we show in Appendix A that they are unstable in the case of angle PD and marginally stable in the case of imaginary part PD.

We now consider the composite coherent communication system as outlined above 
and depicted in Fig. 4-13, using QPSK modulation. The information of this system is in the phase, and thus it is essential to have a reliable and robust phase estimate at the receiver. We see from the analysis of the steady state that the RLS algorithm can not handle significant mean Doppler. This motivates the use of a PLL outside the adaptive RLS receiver to compensate a Doppler shift. If we consider the signal model (4.19) there are $L$ different Doppler shifts. Therefore if the bandwidth of the PLL is small, relative to the Doppler spread made up of the different Doppler shifts, the PLL is at most able to remove one of the Doppler shifts or it locks on to an intermediate frequency. Nevertheless the residual Doppler is left for the filter taps to take care of. Thus we have a situation where both the PLL and RLS see Doppler in their input signals. The RLS steady state analysis shows that the RLS performs a PLL function. The RLS-PLL and the PLL are designed for the same purpose.

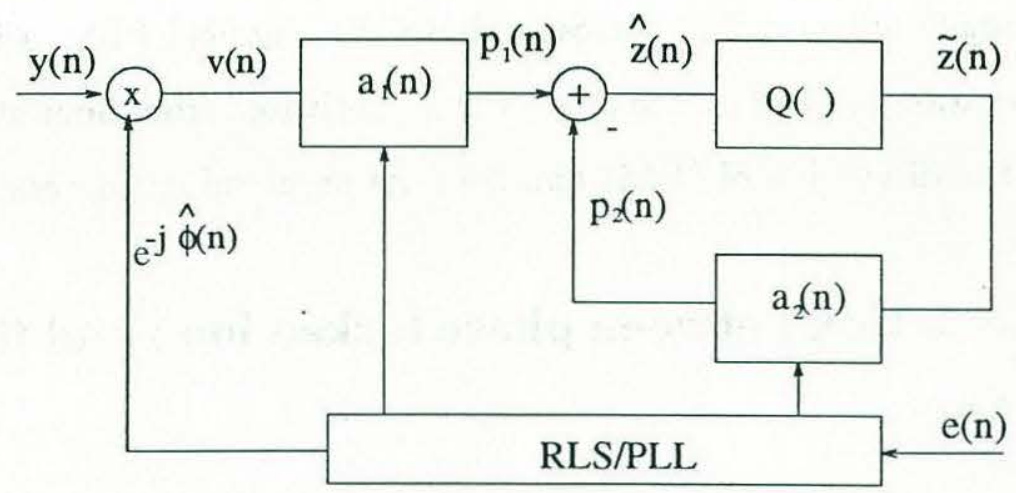

Figure 4-13: The composite receiver, consisting of a decision feedback equalizer and a phase locked loop.

Let us now analyze this situation when the PLL and RLS adaptation of the taps take place on the same data. We constrain ourselves to the simple example treated in Section 4.2.5, and $y(n)$ is given by (4.20). The receiver structure is as in Fig. 4-5 with no feedback taps and a single feedforward tap. In addition a PLL correcting the phase of $y(n)$ is present as shown in Fig. 4-13. 


\section{Difference equations}

We now derive the system of equations that describes the simultaneous adaptation of the PLL and the RLS in presence of Doppler. We then look for stationary regions and limit cycles of the composite system of difference equations from the RLS algorithm and the PLL. As will be shown the angle PD has no stable stationary regions. Thus we derive stationary regions for both systems, but look for limit cycles only in the system comprising the imaginary part PD.

First observe that there is no principal difference between Fig. 4-13 and a corresponding situation where the PLL is absent when it comes to the input signal as seen by the filter $a(n)$. The only difference is that the input signal to the feedforward filter is

$$
v(n)=z(n) e^{j(\phi(n)-\hat{\phi}(n))}
$$

rather than

$$
v(n)=z(n) e^{j \phi(n)} .
$$

Thus the derivation of the filter tap evolution as driven by RLS is not changed. In particular (4.41) is valid:

$$
a(n+1)=\lambda a(n)+(1-\lambda) e^{j(\phi(n)-\hat{\phi}(n))} .
$$

Angle PD The output of the single tap feedforward section of the DFE is

$$
p_{1}(n)=a^{*}(n) v(n)=\hat{z}(n)
$$

because there is no feedback part. By using (4.63), (4.38) and (4.22) we get

$$
\begin{aligned}
p_{1}(n) & =a^{*}(n) z(n) e^{j(\phi(n)-\hat{\phi}(n))} \\
e(n) & =z(n)-\hat{z}(n)
\end{aligned}
$$




$$
\begin{aligned}
p_{1}(n) e^{*}(n) & =p_{1}(n) z^{*}(n)-p_{1}(n) p_{1}^{*}(n) \\
& =a^{*}(n) e^{j(\phi(n)-\hat{\phi}(n))}-|a(n)|^{2} .
\end{aligned}
$$

From this the phase detector output for the angle PD is

$$
\angle\left[p_{1}(n) e^{*}(n)\right]=\angle\left[a^{*}(n) e^{j(\phi(n)-\hat{\phi}(n))}-|a(n)|^{2}\right] .
$$

Since the loop transfer function is $\gamma(n)$ and (4.62) is valid we have the following nonlinear system of difference equations:

$\mathbf{x}(n+1)=\left[\begin{array}{c}a(n+1) \\ \hat{\phi}(n+1)\end{array}\right]=\left[\begin{array}{c}\lambda a(n)+(1-\lambda) e^{j(\phi(n)-\hat{\phi}(n))} \\ \gamma(n) \star \angle\left[a^{*}(n) e^{j(\phi(n)-\hat{\phi}(n))}-|a(n)|^{2}\right]\end{array}\right]=\mathbf{f}(\mathbf{x}(n), \phi(n))$

For simplicity let $\gamma(n)$ be an integrator (the loop filter is 1 ), and then we get

$$
\mathrm{f}(\mathrm{x}(n), \phi(n))=\left[\begin{array}{c}
a(n+1) \\
\hat{\phi}(n+1)
\end{array}\right]=\left[\begin{array}{c}
\lambda a(n)+(1-\lambda) e^{j(\phi(n)-\hat{\phi}(n))} \\
\hat{\phi}(n)+\angle\left[a^{*}(n) e^{j(\phi(n)-\hat{\phi}(n))}-|a(n)|^{2}\right]
\end{array}\right] .
$$

Imaginary part PD The imaginary part PD output is from (4.53) and (4.54)

$$
\operatorname{Im}\left[p_{1}(n) e^{*}(n)\right]=\operatorname{Im}\left[a^{*}(n) e^{j(\phi(n)-\hat{\phi}(n))}\right]
$$

and by letting $\gamma(n)$ be an integrator as with the angle PD, the imaginary part PD give another set of nonlinear coupled difference equations for the filter tap and PLL output:

$$
\mathbf{f}(\mathbf{x}(n), \phi(n))=\left[\begin{array}{c}
a(n+1) \\
\hat{\phi}(n+1)
\end{array}\right]=\left[\begin{array}{c}
\lambda a(n)+(1-\lambda) e^{j(\phi(n)-\hat{\phi}(n))} \\
\hat{\phi}(n)+\operatorname{Im}\left[a^{*}(n) e^{j(\phi(n)-\hat{\phi}(n))}\right]
\end{array}\right] .
$$

The set of nonlinear coupled difference equations are similar to the ones found in [35], [34] where a passband DFE is adapted with a stochastic gradient algorithm 
and a PLL is used on the data at the same time. Some of the conclusions that we make here could also be drawn from this work.

\section{Stationary regions}

The trajectories of $a(n)$ and $\hat{\phi}(n)$ vs $n$ can be drawn in separate coordinate systems to visualize their evolution. Given initial conditions and input $\phi(n)$ the evolution of $\mathrm{x}(n)$ is well defined. We note that the set of difference equations determining the evolution of the tap and the PLL are coupled in both the cases of angle part PD and imaginary part PD in (4.69) and (4.67). We show in the following that it is of interest to determine what we call stationary regions of these difference equations. A stationary region is a set of $\mathrm{x}(n)$ for which the difference equations 1 ) decouples and 2) exhibits fixed point behavior in some of its coordinates. We find in both PD cases that a stationary region exists for $a(n)=e^{j(\phi(n)-\hat{\phi}(n))}$ because by inserting this in (4.69) or (4.67) we have that $\hat{\phi}(n+1)=\hat{\phi}(n)$. Thus $\hat{\phi}(n)$ is constant vs $n$ and we have that

$$
\mathrm{X}_{*}(n)=\left[\begin{array}{c}
e^{j\left(\phi(n)-\hat{\phi}_{0}\right)} \\
\hat{\phi}_{0}
\end{array}\right]
$$

is a stationary region.

\section{Limit cycles}

We now look for limit cycles of the system under the constraint that the phase in the input signal is a linear function of time which corresponds to constant Doppler shift:

$$
\phi(n)=\omega n
$$

The analysis can be carried out by specializing some of the equations. We consider the case of imaginary part PD since it is, as is shown, at least marginally stable. From (4.50) we know that if a constant Doppler $\omega_{0}$ is driving the RLS algorithm we 
have

$$
a(n) \approx \frac{1-\lambda}{1-\lambda e^{j \omega_{0}}} e^{j \omega_{0} n}
$$

Since the PLL and the RLS are interacting we do not know if the RLS will receive the constant Doppler $\omega_{0}$ even if the input signal has a constant Doppler $\omega$. To show that the RLS actually receives a constant Doppler in this case we first assume that this is true: Assume there is a constant Doppler into the RLS i.e., (4.72) is assuming

$$
\phi(n)-\hat{\phi}(n)=\omega n-\omega_{1} n=\omega_{0} n
$$

Here $\omega$ is the total Doppler in the input signal, $\omega_{1}$ is the Doppler compensated by the PLL and $\omega_{0}$ is the residual Doppler present at the input of the RLS. Thus the assumption that (4.72) is true implies (4.73) which assumes that the Doppler compensated by the PLL is constant equal to $\omega_{1}$. We now show that this is true, and thereby make the assumptions of constant Doppler shift into both the PLL and the RLS a consistent pair: The Doppler driving the RLS comes from the PLL output, so the phase output of the PLL must be a linear function of time for (4.72) to be true. Equation (4.69) gives

$$
\hat{\phi}(n+1)=\hat{\phi}(n)+\operatorname{Im}\left[a^{*}(n) e^{j \omega_{0} n}\right]=\hat{\phi}(n)+\operatorname{Im}\left[\frac{1-\lambda}{1-\lambda e^{-j \omega_{0}}}\right] .
$$

Indeed, the PLL is increased by a constant amount at each iteration, so the PLL output is a linear function of time.

As mentioned earlier a limit cycle occurs when the filter tap $a(n)$ repeats itself after a number of steps $n$. This will not be the case with the state $\mathrm{x}(n)$ made up of (4.72) and (4.74) unless

$$
\begin{aligned}
& \omega_{0}=\frac{2 \pi}{k} \\
& \omega_{1}=\frac{2 \pi}{l}
\end{aligned}
$$


for $k, l$ integers. Thus we have shown that

$$
\mathbf{x}(n)=\left[\begin{array}{c}
\frac{1-\lambda}{1-\lambda e^{j \omega_{0}}} e^{j \omega_{0} n} \\
\omega_{1} n
\end{array}\right]
$$

is a limit cycle to (4.69) when (4.75) is satisfied. But even when (4.75) is not satisfied the state trajectory for $a$ is closed which means that the system is in a stable condition, and this is the important feature even if it is not a limit cycle. This is supported by simulation of the system of equations.

\section{Stability analysis}

We now return to the stationary region (4.70), and ask the question whether the stationary region is stable or not. The solution $\mathrm{x}_{*}$ given in (4.70) is valid for both angle PD and imaginary part PD and a stable stationary region would imply that $\mathbf{x}(n)$ in the vicinity of $\mathbf{x}_{*}$ would be attracted to $\mathbf{x}_{*}$ and the trajectory would end up in $\mathrm{x}_{*}$. The stability is determined by linearizing $\mathrm{f}(\mathrm{x})$ around the stationary region and examining the eigenvalues of the resulting matrix. This gives local behavior only since the system is nonlinear.

Consider a perturbation of $\mathrm{x}$ away from the stationary region $\mathrm{x}_{*}$. We can linearize the system by a first order Taylor series of $\mathbf{f}(\mathbf{x})$ around $\mathbf{x}_{*}$, and if the linear system is stable we have a stable stationary region.

$$
\begin{aligned}
\delta \mathrm{x}(n+1) & =A \delta \mathrm{x}(n)=A^{n} \delta \mathrm{x}_{0} \\
A & =\frac{\partial \mathrm{f}}{\partial \mathrm{x}} \mid \mathrm{x}_{*} \\
\text { Stability } & :\left|\kappa_{i}(A)\right|<1 \quad \forall i
\end{aligned}
$$

where $\kappa_{i}(A)$ is the $i^{\prime}$ th eigenvalue of $\mathrm{A}$, and expressions for these are given in Appendix $\mathrm{A}$ in both the cases of angle PD and imaginary part PD. By using a perturbation with arbitrary direction $\delta a=r e^{j \phi}$, and letting $r$ go to zero, the lineariza- 
tion shows that the stationary region is unstable using the angle PD (4.65), and marginally stable (one eigenvalue has magnitude one for a specific direction) for the imaginary part PD (4.68). The reader is referred to Appendix A for details.

In the case of using the angle PD the state trajectory fails to end in the stationary region when started from arbitrary initial conditions since the stationary region is unstable. This forces the system to find another way to go, and the result is a limit cycle. The limit cycle state trajectory and the number of time steps $n$ in a limit cycle is determined in part by the loop filter of the PLL. In the case of a system described by (4.67) the result is a limit cycle with period 2, i.e.,

$$
a(n+2)=a(n) \quad \hat{\phi}(n+2)=\hat{\phi}(n)
$$

and it is possible to find expressions for the limit values by solving

$$
\mathbf{x}(n)=\mathbf{f}(\mathbf{f}(\mathbf{x}(n), \phi(n)))
$$

The period of two in the limit cycle is specific for the loop filter that is assumed in (4.67), and limit cycles of arbitrary period occurs by changing this filter.

Summary We use the steady state RLS solution and the recursion obeyed by the PLL to get a coupled set of non-linear difference equations in (4.67) and (4.69). We find stationary regions and limit cycles of these equations and we analyze their stability. A major point in our context is that the stationary region given by (4.70) is not a single point, but a region. We find that the angle PD has an unstable stationary region, and that the imaginary part $\mathrm{PD}$ has an infinite number of marginally stable limit cycles. Thus the operation of the PLL and filter tap adaptation is not well conditioned. Adding noise and modifying the PLL loop filter further changes the behavior of the system, but the main features discussed above prevail. 


\subsubsection{Simulation and real data examples}

The limit two-cycle for the angle PD in (4.79) is verified by simulations. Assume that we start the system in equilibrium, so that (4.70) is satisfied, and then at time zero we perturb it with

$$
\delta \mathrm{x}_{0}=\left[\begin{array}{c}
\delta a \\
0
\end{array}\right]
$$

where $\delta a$ is a random variable with jointly Gaussian real and imaginary parts of variance 0.1. The trajectories of $a(n)$ in the complex plane are shown in Fig. 4-14. The upper panel shows ten trials of the output $\hat{\phi}(n)$ of the PLL vs time. We see that $\hat{\phi}(n)$ goes into the limit 2-cycle with the two values being symmetric around 0 radians. This symmetry can be verified by looking at (4.67) because the lower equation for $\hat{\phi}(n+1)$ is a difference between $\hat{\phi}(n)$ and the PLL input. Difference equations of this kind are satisfied by symmetric $\hat{\phi}(n)$ with period 2. The lower panel of Fig. 4-14 shows the filter tap $a(n)$ in the complex plane, and in each of the ten trials it is started on the unit circle in accordance with (4.70). The perturbation causes $|a(n)|$ to decrease, and $a(n)$ ends up in limit cycles with $|a(n)|<1$. This is in accordance with (4.67) which states that $a(n)$ is the output of the IIR low pass filter discussed in an earlier section. The behavior of the system is modified by the introduction of a loop filter, but not fundamentally. Fig. 4-15 shows trajectories of $\hat{\phi}(n)$ and $a(n)$ when

$$
\begin{aligned}
& \phi(n)=0.01 n \\
& \Gamma(z)=10^{-3} \frac{1-0.9 z^{-1}}{\left(1-z^{-1}\right)^{2}}
\end{aligned}
$$

where $\Gamma(z)$ is the $z$-transform of the loop transfer function $\gamma(n)$. The phase is linear (constant Doppler) and the loop filter is more complex, and in this example the angle PD is used in the PLL. In this case the real part steady state tap value $a(n)$ in the lower panel of Fig. 4-15 always comes approximately back to one, but the 


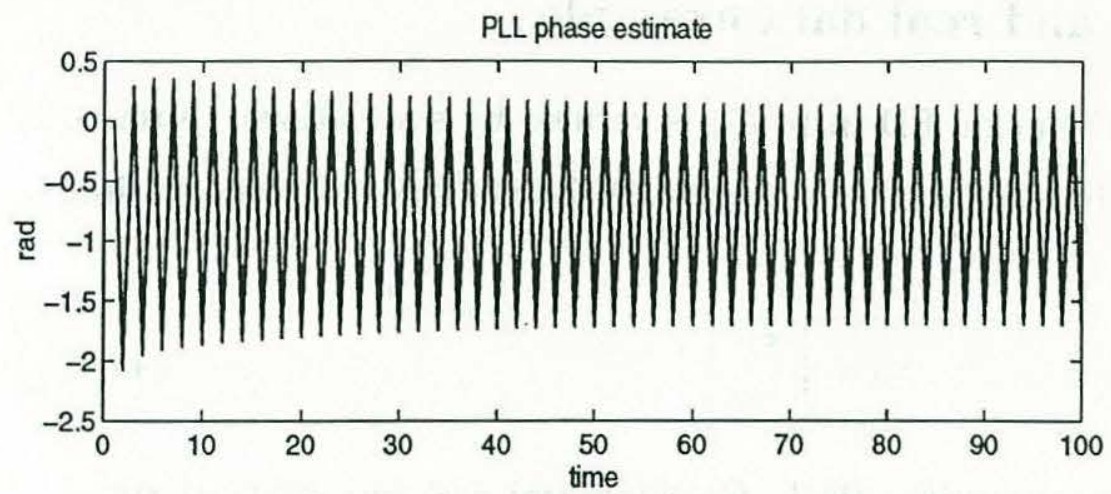

Filter tap $a(n)$

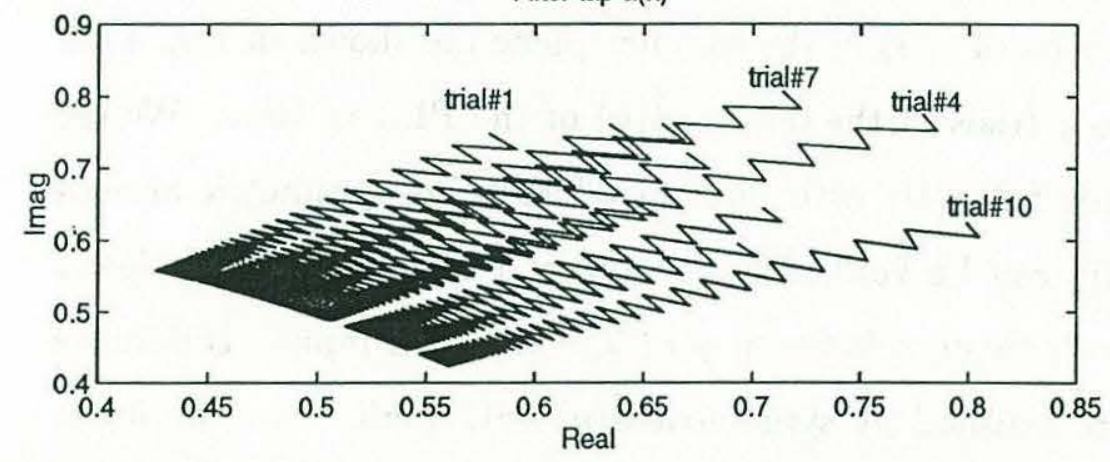

Figure 4-14: 10 trials of the state trajectories when perturbing the filter tap with a small random value $\delta a$ away from the stationary region. The top picture is PLL phase estimate, and the bottom picture is the complex filter tap values.

imaginary part has arbitrary final value so that $|a(n)|$ fails to return to one after a perturbation.

Examples of limit cycles in the case of constant Doppler and imaginary part PD is shown in Fig. 4-16. It shows the filter tap and the PLL output for a linear Doppler input, and in this is picture we can see the action of the IIR filter very clearly. As the PLL locks on to an arbitrary amount of the full Doppler present in the signal the RLS must take care of the rest by means of tap rotation. When the tap rotation is fast the low pass filter attenuates $|a(n)|$ so that it stays away from the unit circle where the stationary regions are.

Also included is an example of RLS and PLL simultaneous adaptation on real data that illustrates the phenomena of the undetermined and unstable stationary 

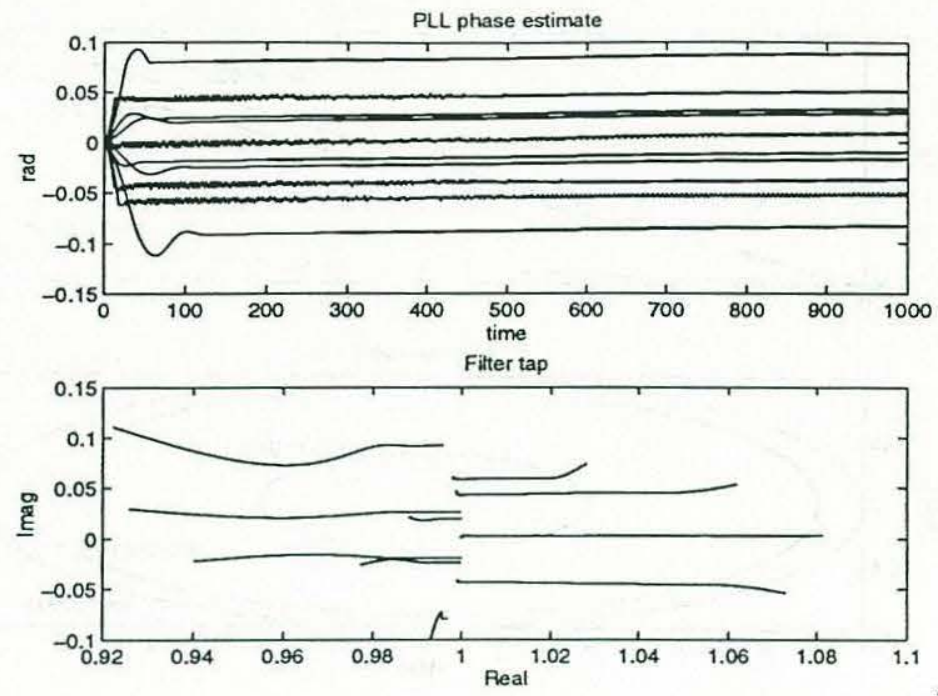

Figure 4-15: 10 trials of the state trajectories when a linear phase shift is applied to the input and the filter tap is perturbed away from the stationary region. The PLL phase estimate at the top, and the complex filter tap values at the bottom. The time arrows of all the trajectories of $a(n)$ are pointing toward the middle where the $\operatorname{Re}(a)=1$.

region of the system. The underwater communication channel used for transmissions consists of one main stable return, and a more rapidly varying delayed return. The receiver has 4 piezo-electric elements receiving the sound field, and they are mounted on a rigid platform spanning less than 1 meter. The platform is towed from a boat, and each of the data channels are connected to a receiver as shown in Fig. 4-17 with one PLL for each data channel. The data channels in Fig. 4-17 are created by complex demodulation and sampling of each of the four transducer outputs, and the sampling rate is 2 samples per symbol. Thus each transducer is equivalent of one data channel of the same kind as is used for input in the previous section. The RLS works jointly on all DFE coefficients to minimize the error. In this configuration it is very unlikely that there should be any difference in Doppler among the data channels. The data are correctly decoded, so the receiver achieves convergence to steady state as assumed in the analysis above. Fig. 4-18 shows the PLL outputs for the data channels, and the left part is when all data channels are used whereas 

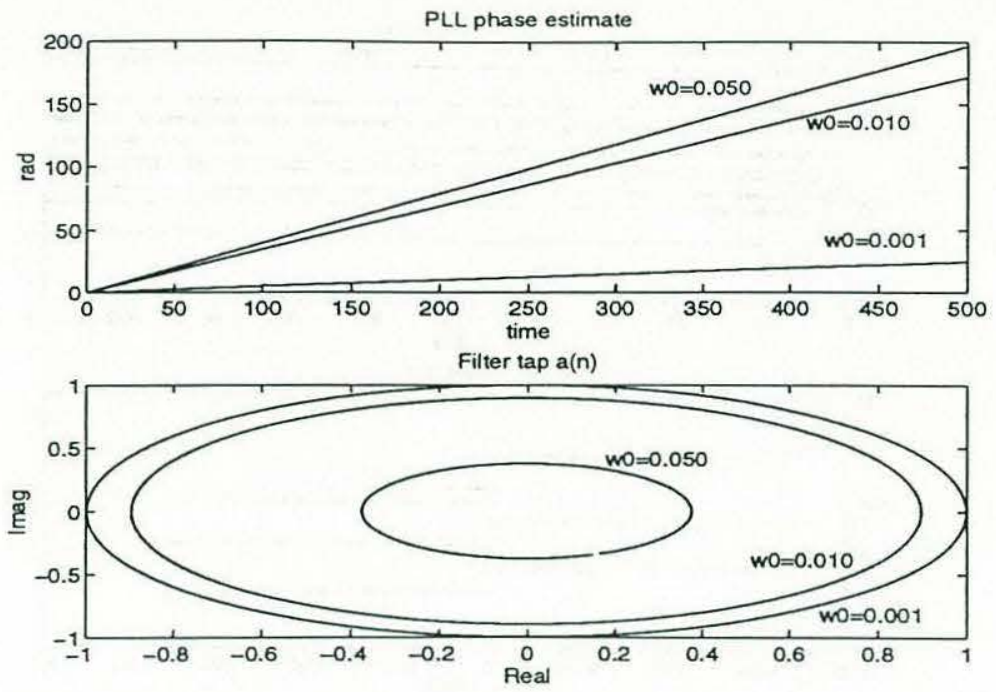

Figure 4-16: The filter tap rotates at a constant rate, taking care of some of the Doppler at the expense of its amplitude. The rest of the Doppler is compensated by the PLL. It is arbitrary how the Doppler is shared between the PLL and the filter tap. There are three cases of Doppler handled by the RLS, $\omega_{0}=\left[\begin{array}{lll}.001 & .01 & .05\end{array}\right]$.

the right part is with two channels used (decoding is still correct). The two lowest curves in the left picture are the same data channels as the two curves in the right picture. Thus the phase estimates on the same data channels are different, and this is due to the ill behaved dynamics of the interaction between the PLL and the filter tap adaptations. The connection between tap amplitude attenuation in (4.50) and Doppler handled by the RLS is shown for this real data example in Fig. 4-19. The tap with the smallest Doppler, corresponding to the channel with largest PLL phase estimate in Fig. 4-18, is the one with largest magnitude in Fig. 4-19. This is in accordance with (4.50) which suggests that the tap amplitude is attenuated when the Doppler compensated by the RLS is significant.

Summary We demonstrate the behavior of the receiver in Fig. 4-13 by simulating the difference equations (4.67) and (4.69) for different cases of Doppler spread. In the case of a Doppler shift the receiver is compensating some fraction of this by means of the RLS and the rest by means of the PLL, and this results in attenuation 


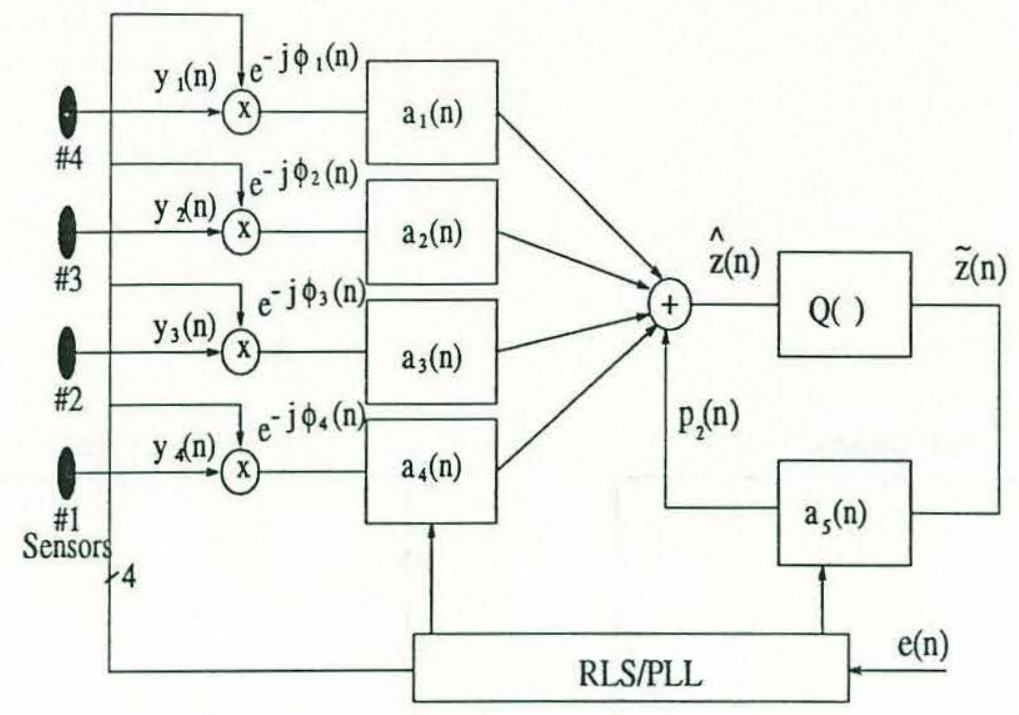

Figure 4-17: The multichannel version of the DFE, running on four data channels. The RLS is jointly adapting all filter coefficients.

of the tap amplitude estimated by the RLS. The system behavior as predicted by (4.67) is verified by looking at the receiver RLS and PLL output when running on real data in Fig. 4-18 and Fig. 4-19. 

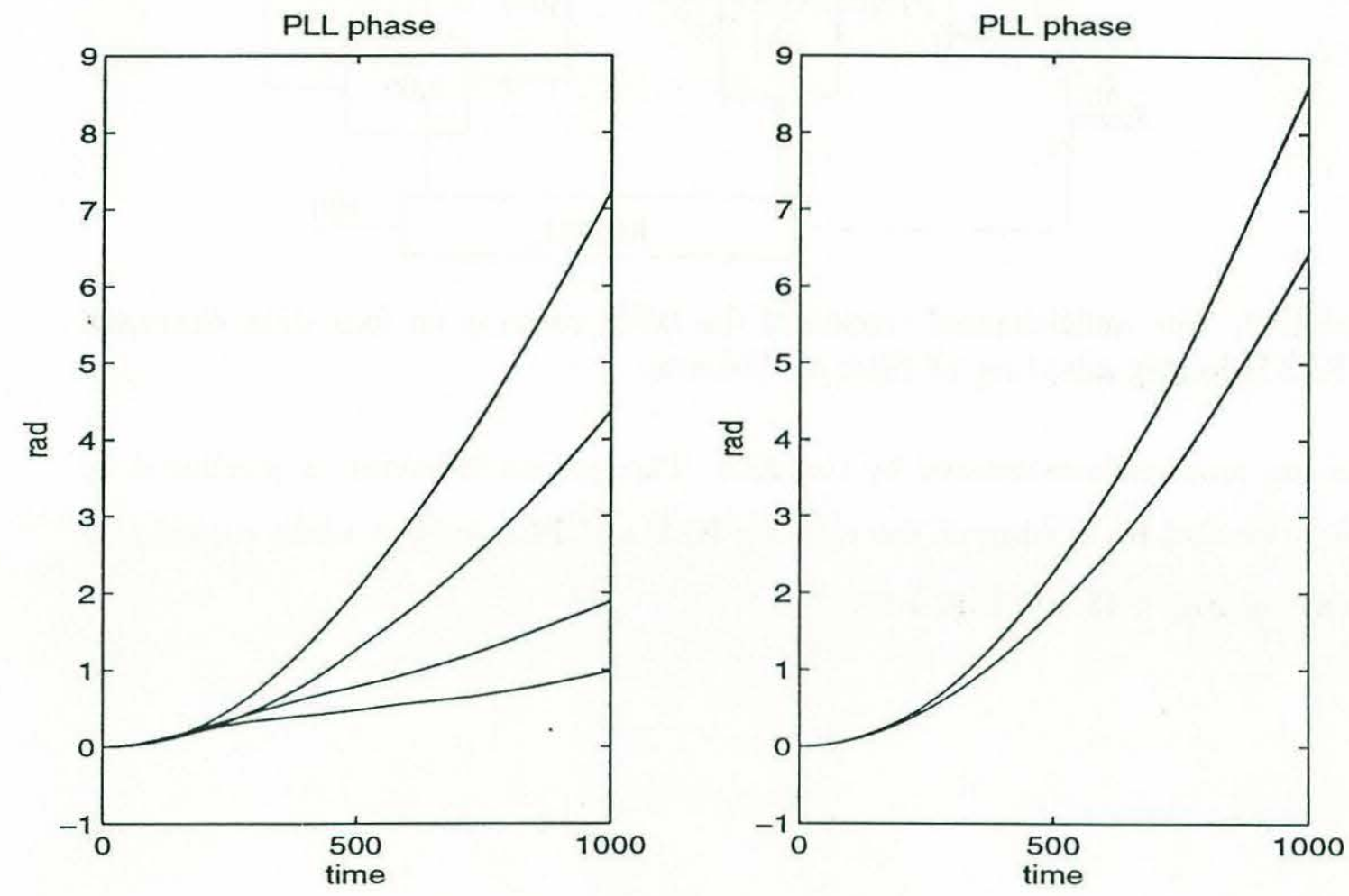

Figure 4-18: The PLL output phase estimate $\hat{\phi}(n)$ when applied to channels 1-4 (left) and channels 1-2 (right) of real data. The four channels are believed to have the same Doppler because they were recorded from elements closely spaced and mounted on a rigid platform, but the PLL estimates are different. The two channels 1 and 2 are estimated differently when channel 3 and 4 are not used. 


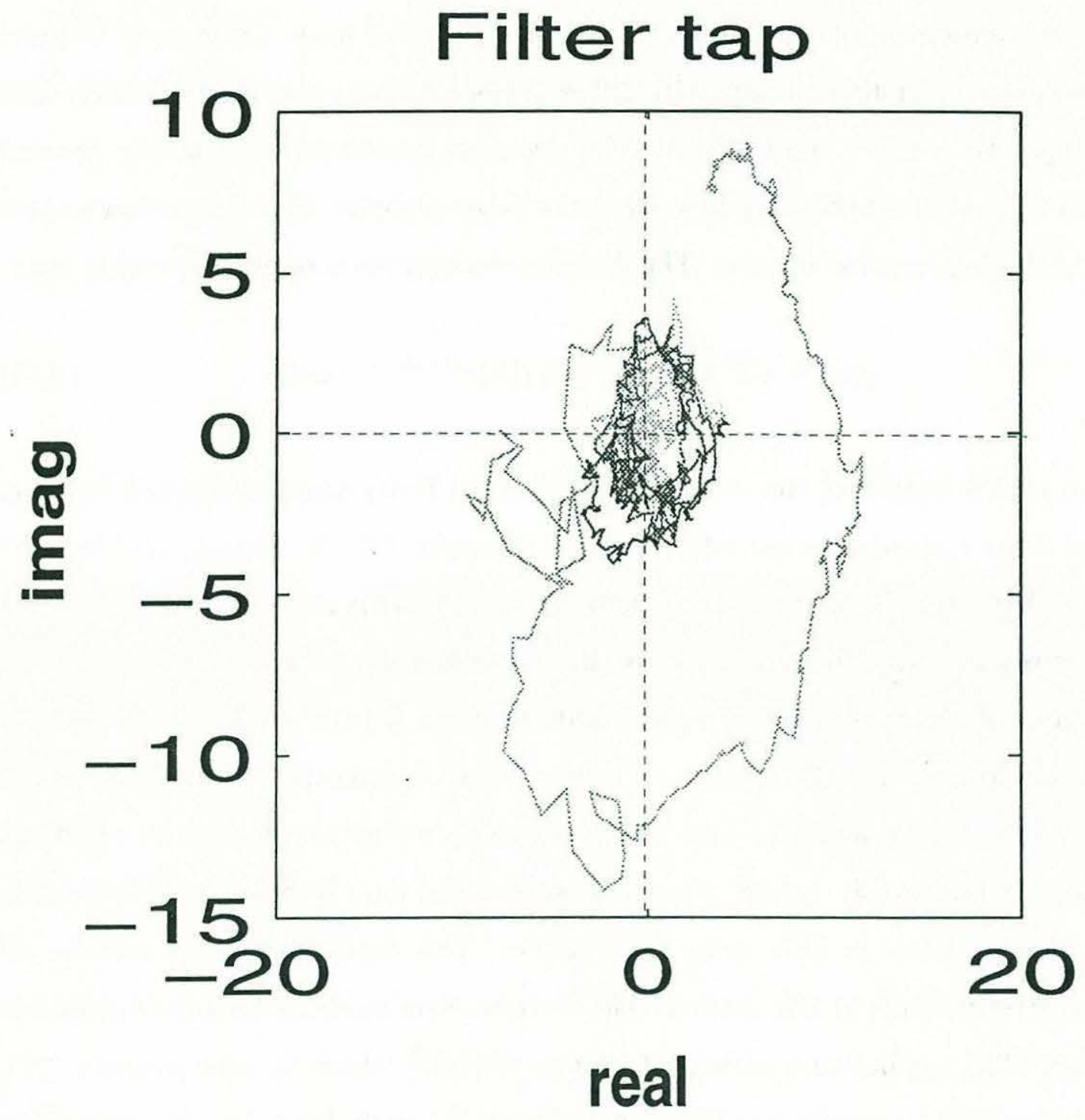

Figure 4-19: The taps of the 4 channel equalizer running on real data. All taps are rotating. The magnitudes of the taps are significantly different, even though the noise has similar variance on all the channels. The highest magnitude tap is on the channel where the PLL phase estimate is largest. 


\subsection{A receiver for doubly spread channels}

\subsubsection{Channel model}

We now propose a receiver that works on a sparse channel in the presence of Doppler spread made up of different rays with different Doppler shifts that may be slowly time varying. We consider the modified delay-Doppler-Spread function as our channel model where the modification is to allow the delay-Doppler-spread function to be a slowly varying function of time. The discrete representation of our channel is thus

$$
y(n)=\sqrt{\mathcal{E}} \sum_{(l, k)}^{L} z(n-l) U_{l, k}(n) e^{j 2 \pi k \Delta \nu n}+w(n)
$$

where $y(n)$ is output of the channel, $U_{l, k}(n)$ is the Delay-Doppler-Spread function which is the scattering amplitude at lag $l$ and Doppler $k \Delta \nu$ for time $n, z(n)$ is input to the channel, $L$ is the number of signal returns, $w(n)$ is measurement noise, $\mathcal{E}$ is the energy in each symbol and $\Delta \nu$ is the Doppler spacing. The notation $(l, k)$ in the index of the sum of (4.83) means that there are $L$ pairs of $(l, k)$, and there is no assumption on the distribution of these points. Specifically, some of the delays $l_{i}$ may be identical and also some of the Doppler coefficients $k_{i}$ may be identical. Thus we model doubly spread, pure delay spread and pure Doppler spread channels that may be sparse in both delay and Doppler. This channel model is used for all the receiver variants in this section. The basic receiver model, which is discussed in Section 4.3.3, applies to a restricted version of (4.83) which we now present. The restriction is to have only one Doppler coefficient for each delay, i.e., for each delay $l$ there is only one $k$. By collecting $z(n)$ in the vector $\mathbf{c}^{H}(n)$ we can write (4.83) as

$$
y(n)=\mathbf{c}^{H}(n)\left[\begin{array}{ccc}
e^{j 2 \pi k_{0} \Delta \nu n} & & 0 \\
& \ddots & \\
0 & & e^{j 2 \pi k_{L-1} \Delta \nu n}
\end{array}\right]\left[\begin{array}{c}
U_{l_{0}, k_{0}}(n) \\
\vdots \\
U_{l_{L-1}, k_{L-1}}(n)
\end{array}\right]+w(n)
$$




$$
\mathbf{c}^{H}(n)=\sqrt{\mathcal{E}}\left[z\left(n-l_{0}\right) \cdots z\left(n-l_{L-1}\right)\right] .
$$

Now we define a state space description for a system aiming at deriving a recursive estimate of the state space vector $\mathbf{h}(n)$ :

$$
\mathbf{h}(n)=\left[\begin{array}{c}
U_{l_{0}, k_{0}}(n) e^{j 2 \pi k_{0} \Delta \nu n} \\
\vdots \\
U_{l_{L-1}, k_{L-1}}(n) e^{j 2 \pi k_{L-1} \Delta \nu n}
\end{array}\right] .
$$

We assume a first order autoregressive $(\operatorname{AR}(1))$ model for $U_{l_{i}, k_{i}}(n)$, so that

$$
U_{l_{i}, k_{i}}(n+1)=\alpha_{i} U_{l_{i}, k_{i}}(n)+\tilde{v}_{i}(n) \quad 0 \leq i \leq L-1
$$

where $\left|\alpha_{i}\right| \leq 1$ and $\tilde{v}_{i}(n)$ is white noise. There is one $\alpha_{i}$ for each pair $\left(l_{i}, k_{i}\right)$. The original delay-Doppler-spread model of $U_{l_{i}, k_{i}}(n)$ being constant random variables (not functions of time $n$ ) is contained in this model when the noise variance is zero and $\alpha_{i}=1$. The state space model for $\mathbf{h}(n)$ is now given by

$$
\begin{aligned}
\mathbf{h}(n+1) & =A \mathbf{h}(n)+\mathbf{v}(n) \\
A & =\left[\begin{array}{ccc}
\alpha_{0} e^{j 2 \pi k_{0} \Delta \nu} & & 0 \\
& \ddots & \\
0 & \alpha_{L-1} e^{j 2 \pi k_{L-1} \Delta \nu}
\end{array}\right] \\
y(n) & =\mathbf{c}^{H}(n) \mathbf{h}(n)+w(n)
\end{aligned}
$$

where

$$
\mathbf{v}=\left[v_{0}(n) \cdots v_{L-1}(n)\right]^{T}
$$




$$
\begin{aligned}
R & =E\left[\mathrm{vv}^{H}\right]=\left[\begin{array}{ccc}
\left(1-\alpha_{0}^{2}\right) u_{0}^{2} & & 0 \\
& \ddots & \\
0 & & \left(1-\alpha_{L-1}^{2}\right) u_{L-1}^{2}
\end{array}\right] \\
u_{i}^{2} & =E\left[\left|U_{l_{i}, k_{i}}\right|^{2}\right] \\
v_{i}(n) & =\tilde{v}_{i}(n) e^{j 2 \pi k_{i} \Delta \nu(n+1)} .
\end{aligned}
$$

The quantity $u_{i}^{2}$ is the scattering strength at $\left(l_{i}, k_{i}\right)$, and the introduction of $v_{i}(n)$ is merely a notational convenience. We note that if the assumption of one Doppler per delay is not satisfied, the resulting state space model is derived from

$$
y(n)=\sum_{l_{i}} \sum_{k_{i}} U_{l_{i}, k_{i}}(n) e^{j 2 \pi k_{i} \Delta \nu n}
$$

The coefficients $U_{l_{i}, k_{i}}(n)$ are only observed through their sum over $k_{i}$, and it is possible to resolve this sum only if we have a number of time samples $y(n) \cdots y(n+$ $N-1)$. Even in the case of one Doppler per delay we use on the order of $N \gg$ $L$ samples, where $N$ is given by the exponential weighting $\lambda$ to be introduced in Section 4.3.2. The reason for large $N$ in this case is to get noise suppression through averaging as discussed in Section 4.1.5. In the case of (4.89) we need $N$ large in order to resolve the sum over $k_{i}$, and this also depends on $\Delta \nu$. This is used when the basic receiver is extended, and we comment further on this in Section 4.3.4. The diagonal form of $A$ is obtained because we model the scattering from different lags and at different Dopplers as being uncorrelated with each scattering process being WSS, and this corresponds to the WSSUS assumption that is discussed in Section 2.2. The WSSUS assumption also implies a diagonal form of $R$. The channel model (4.87) is used to derive a receiver which also makes use of a modified RLS algorithm that we present in Section 4.3.2. 
Summary We use the time-variant delay-Doppler-spread function $U_{l, k}(n)$ as our channel model in (4.83). The time evolution of $U_{l, k}(n)$ is modeled as an $\operatorname{AR}(1)$ process in (4.86) and by means of this a state space model (4.87) is presented.

\subsubsection{The modified RLS algorithm}

The regular RLS algorithm is given in (4.22) and (4.23), and it can be shown [64] that it minimizes a weighted sum of the errors given by

$$
\sum_{m=0}^{n} \lambda^{n-m}|e(m)|^{2}
$$

When the forgetting factor $\lambda=1$ the RLS is identical with a Kalman filter [45], [64] for the system with state space description

$$
\begin{aligned}
\mathbf{h}(n+1) & =\mathbf{h}(n) \\
y(n) & =\mathbf{c}^{H}(n) \mathbf{h}(n)+w(n)
\end{aligned}
$$

where the variance of $w(n)$ is $\sigma_{\cdot w}^{2}=1$ for the analogy to hold. Thus in Kalman filter terminology [105] the system matrix of (4.91) is the identity matrix $I$ and the observation matrix $C$ is the data vector $\mathbf{c}(n)$. The analysis in Section 4.2 on the DFE identified one of the main problems as a model mismatch between the true channel model and the underlying model for the RLS given by (4.91). The mismatch in this case is in the time update of (4.91) where the model (4.87) predicts a rotation of $\mathbf{h}$. Motivated by this, and also by findings in the literature [46] that address the same issue, we introduce a modified RLS with an improved model for the time update that incorporates the model (4.87) and (4.88). Thus we replace the regular RLS (4.22) with

$$
\hat{\mathbf{h}}(n+1)=A \hat{\mathbf{h}}(n)+\mathbf{k}(n) e(n)
$$




$$
\begin{gathered}
\hat{y}(n)=\mathbf{c}^{H}(n) A \hat{\mathbf{h}}(n) \\
\mathbf{c}^{H}(n)=\sqrt{\mathcal{E}}\left[z\left(n-l_{0}\right) \cdots z\left(n-l_{L-1}\right)\right] \\
e(n)=y(n)-\hat{y}(n) \\
\mathbf{k}(n)=\frac{\frac{1}{\lambda}\left(A P(n-1) A^{H}+R\right) \mathbf{c}(n)}{\sigma_{w}^{2}+\frac{1}{\lambda} \mathbf{c}^{H}(n)\left(A P(n-1) A^{H}+R\right) \mathbf{c}(n)} \\
P(n)=\frac{1}{\lambda}\left(A P(n-1) A^{H}+R\right)-\frac{1}{\lambda} \mathbf{k}(n) \mathbf{c}^{H}(n)\left(A P(n-1) A^{H}+R\right)
\end{gathered}
$$

where $w(n)$ has variance $\sigma_{w}^{2}$ and $e(n)$ is the innovations sequence. We call this algorithm the time updated RLS (TU-RLS) and in (4.93) $\mathrm{k}(n)$ is the gain vector and $P$ is a covariance matrix. If $\lambda=1$ we have that $\mathrm{k}$ is the Kalman gain and $P$ is the prediction error covariance matrix. This recursive algorithm is also reminiscent of the RLS since it degenerates to the regular RLS when the Doppler spread is zero, $w(n)$ has unit variance and $R=0$, and it could be called the Doppler spread RLS. The Doppler spread enters the TU-RLS through the $A$ matrix which yields differential Doppler among ray paths, and also through $R$ which yields the effect of a time-variant ray. Additional motivation for this modified RLS is revealed as we use the algorithm and analyze the results in Section 4.3.3-4.3.8. We now combine the TU-RLS and the channel model (4.87) to present the receiver.

Summary We propose a modified RLS algorithm with a time update step (TURLS) that incorporates the channel model (4.87) in (4.92) and (4.93). This recursive algorithm is identical to the regular RLS when the Doppler spread is zero, $w(n)$ has unit variance and $R=0$. It is identical to a Kalman filter when the exponential weighting $\lambda$ is one and $w(n)$ had unit variance. 


\subsubsection{Receiver for one Doppler shift at each delay}

Consider the receiver design in fig. $4-20$, which is built according to a demand for simultaneous channel tracking and coherent signal combining to reduce dispersion in both time and frequency. The system for channel tracking obeys the TU-

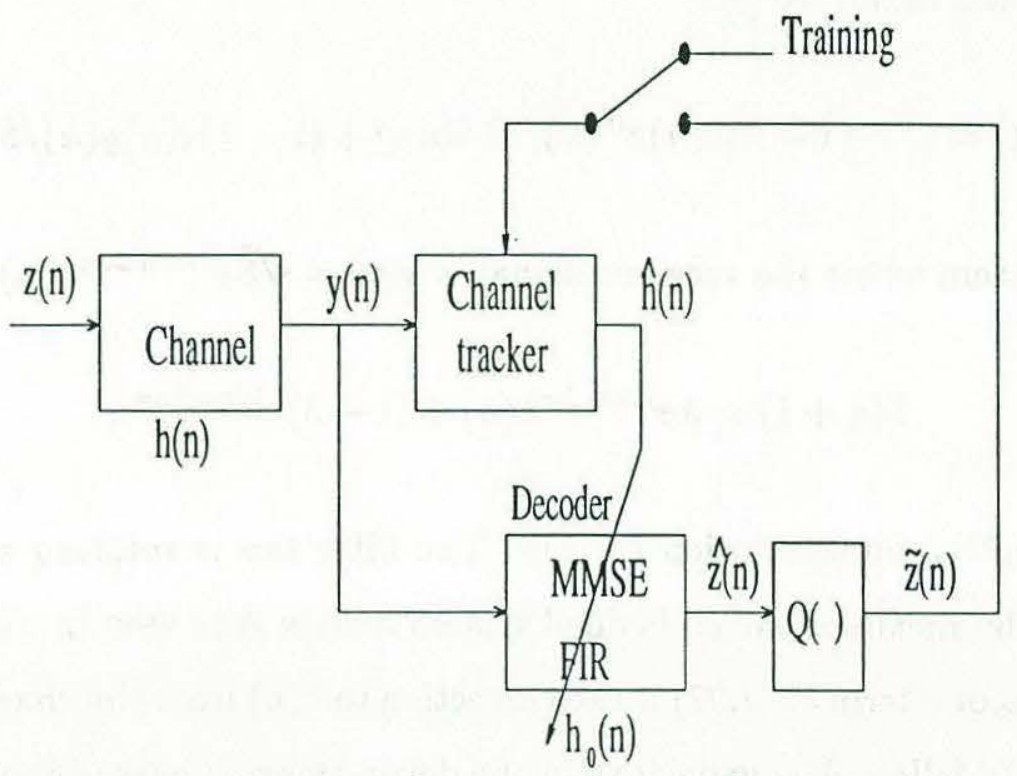

Figure 4-20: Receiver built up of separate channel tracker and equalizer.

RLS algorithm yielding a recursive estimate of $h(n)$ and a recursive equation for the covariance $P(n)$. To obtain the algorithm we apply the TU-RLS to the model (4.87), and this yields the estimate $\hat{h}(n)$ in (4.92) and a covariance matrix and gain vector varying according to (4.93).

Before we characterize the receiver we now compare the tracking part of the receiver in Fig. 4-20 to the DFE in Fig. 4-13 with respect to Doppler. In order to make the comparison as relevant as possible we remember that one of the channel models used with the DFE is a time-invariant delay-Doppler-spread function. To accommodate this we require

$$
\alpha_{i}=1 \quad \forall i
$$

so that we can look for a steady state solution of the covariance equation in the same 
way as for the DFE in Section 4.2. We show in Appendix B that the gain for this case is given by (B.6), (B.7) and (B.8) which yields

$$
\mathrm{k}(n) \approx(1-\lambda) \mathrm{c}(n) / \mathcal{E}
$$

Using (4.92) and (4.95) we get

$$
\hat{\mathbf{h}}(n+1)=\left[I-(1-\lambda) \mathbf{c}(n) \mathbf{c}^{H}(n) / \mathcal{E}\right] A \hat{\mathbf{h}}(n)+(1-\lambda) \mathbf{c}(n) y(n) / \mathcal{E} .
$$

In a $1 \times 1$ system where the received signal is $y(n)=\sqrt{\mathcal{E}} e^{j 2 \pi k_{0} \Delta \nu n} z(n)$ we get

$$
\hat{h}(n+1)=\lambda e^{j 2 \pi k_{0} \Delta \nu} \hat{h}(n)+(1-\lambda) e^{j 2 \pi k_{0} \Delta \nu n} .
$$

Note the intuitive interpretation of this: The filter tap is rotating at the correct Doppler rate by means of the embedded system matrix $A$ as seen in the first term of (4.97). The second term of (4.97) is the correction to $\hat{h}(n)$ from the channel variation. The tracking to follow slow variations in the delay-Doppler-spread function is still a first order IIR filter, refer to (4.41), but now the Doppler frequency is not entering the filter. The first order IIR filter is not a lowpass filter but a bandpass filter centered approximately at the Doppler frequency, and this is the effect of the TU-RLS.

The tap in (4.97) rotates in the same direction as the channel whereas the tap in (4.41) rotates in the opposite direction of the channel. This is consistent with the fact that the DFE (4.41) directly compensates the channel whereas the TU-RLS (4.97) only tracks the channel.

Summary We present the basic receiver architecture in Fig. 4-20, and it comprises a channel tracker updated with TU-RLS, a linear decoder and a quantizer. We show the effect of a simple Doppler shift on the TU-RLS in (4.97) and compare it to the DFE in Section 4.2. 


\subsubsection{Generalized receiver}

We now consider a way to increase the amount of Doppler spread that can be present at a given delay. The state space model that is used to derive the channel tracker in Fig. 4-20 has only one nonzero Doppler coefficient at each delay, and we now discuss the situation where several values of the delay-Doppler-spread function at a specific delay are nonzero. This can be accommodated by a modified receiver which we now present.

The signal model is given by (4.83) and in this section we assume $\mathcal{E}=1$. In the receiver in Fig. 4-20 we assume correct decoding and use past decisions $\tilde{z}(n)$ to track the channel. The channel variation is assumed to be much slower than the symbol rate, therefore we can introduce a small delay in the channel tracking and thereby have the transmitted sequence $z(n)$ available. As will be further explained below this enables us to bring out single terms in the delay index of (4.83) by assuming that $z(n)$ is an uncorrelated sequence, and at a specific delay $l_{0}=0$ we can write

$$
\begin{aligned}
U_{0, k_{0}}(n) & =U_{k}(n) \\
y_{0}(n) & =z(n) \sum_{k}^{K_{0}-1} U_{k}(n) e^{j 2 \pi k \Delta \nu n}+w(n) .
\end{aligned}
$$

The physical interpretation of (4.98) is $K_{0}$ rays with Doppler shifts $k \Delta \nu$. Each ray is time variant since $U_{k}(n)$ is a function of time. The Doppler spread on a ray is

given by the bandwidth $B_{k}$ of $U_{k}(n)$. We now assume that there is only one ray with coefficient $U_{0}(n)=U(n)$ present at $k=0$ so that (4.98) yields

$$
y_{0}(n)=z(n) U(n)+w(n) .
$$

Thus $U(n)$ is the time-variant gain of a single ray, and this ray is the only path for signal propagation in this particular channel. In the context of the general channel model (4.83) we have that $L=1$ and $l_{0}=k_{0}=0$. We observe the sequence $U(n)$ 
over a finite time $N$ and thus $U(n)$ can be represented by its Fourier transform $\tilde{U}_{k}$

$$
y_{0}(n)=z(n) \sum_{k=0}^{K-1} \tilde{U}_{k} e^{j 2 \pi k \Delta \nu n}+w(n)
$$

where we assume that the number of non-zero Fourier coefficients is $K \ll N$.

\section{Block processing}

We now derive an estimator for the coefficients $U_{l, k}$ in our signal model (4.83). In the derivation of the receiver in Fig. 4-20 it is argued that the channel estimation and the symbol decoding can be carried out separately. The selection of the taps $\{(l, k)\}$ to track is carried out by means of the training sequence, and it is treated in Section 4.3.6. We now assume that this initialization has been performed and that we are in tracking mode. By introducing a small delay and assuming correct decoding $z(n)$ is known, and we have a situation where $y(n), z(n)$ and $\Delta \nu$ are all known and the task is to find $U_{l, k}$. We now define the vectors

$$
\begin{aligned}
\mathbf{y} & =[y(m) \cdots y(m+N-1)]^{T} \\
\mathbf{w} & =[w(m) \cdots w(m+N-1)]^{T}
\end{aligned}
$$

where $\mathrm{w}$ is a zero-mean white Gaussian random vector with covariance matrix $\sigma_{w}^{2} I$. The probability density of $\mathrm{y}$ conditioned on $\mathrm{h}$ given by (4.85) is

$$
\begin{aligned}
p_{\mathbf{y} \mid \mathbf{h}}= & \frac{1}{\left(\pi \sigma_{w}^{2}\right)^{N}} e^{-\left(\mathbf{y}-\mathbf{m}_{\mathbf{y} \mid \mathbf{h}}\right)^{H}\left(\mathbf{y}-\mathbf{m}_{\mathbf{y} \mid \mathbf{h}}\right) / \sigma_{w}^{2}} \\
\mathbf{m}_{\mathbf{y} \mid \mathbf{h}}= & {\left[\sum_{(l, k)} z(m-l) U_{l, k} e^{j 2 \pi k \Delta \nu m} \cdots \sum_{(l, k)} U_{l, k} z(m+N-1-l) \times\right.} \\
& \left.e^{j 2 \pi k \Delta \nu(m+N-1)}\right]^{T} .
\end{aligned}
$$


The ML estimate of $h$ is

$$
\begin{aligned}
\hat{\mathbf{h}} & =\min _{\mathbf{h}} \sum_{n=m}^{m+N-1}\left|y(n)-\sum_{(l, k)} z(n-l) U_{l, k} e^{j 2 \pi k \Delta \nu n}\right|^{2} \\
& =\min _{\mathbf{h}} \sum_{n=m}^{m+N-1}|e(n)|^{2} .
\end{aligned}
$$

In order to find the minimum we solve

$$
\partial \sum_{n=m}^{m+N-1}[\cdot] / \partial U_{i, p}=0
$$

We note that (4.104) implies the derivative of the nonanalytic, real quantity in the minimization of (4.103) with respect to the complex variable $U_{i, p}$. This is interpreted in slightly different ways [45], [13] [85]. We use the interpretation of [13] in carrying out the derivative in (4.104) which yields

$$
\sum_{n=m}^{m+N-1} y(n) z^{*}(n-i) e^{-j 2 \pi p \Delta \nu n}=\sum_{\substack{n=m \\ j 2 \pi(k-p) \Delta \nu n}}^{m+N-1} \sum_{\substack{(l, k) \\ j 2 \pi}} z(n-l) z^{*}(n-i) U_{l, k} \times
$$

for the $L$ pairs $(i, p)$. This can be solved for $U_{l, k}$ assuming that the $L \times L$ matrix with elements $l, k$ given by the sum in the curly brackets of (4.106) is invertible. An approximate solution can be obtained by noting that the right hand side of (4.105) yields

$$
N \sum_{(l, k)} U_{l, k}\left\{\frac{1}{N} \sum_{n=m}^{m+N-1} z(n-l) z^{*}(n-i) e^{j 2 \pi(k-p) \Delta \nu n}\right\}
$$

and identifying the term in curly brackets as the sample correlation between $z(n-l)$ and $z^{*}(n-i) e^{j 2 \pi(k-p) \Delta \nu n}$. This will approach zero for large $N$ when $l \neq i$ because $z(n)$ is an uncorrelated sequence, and also for $l=i, k \neq p$ if we use $\Delta \nu=1 / N$. We note that this may not always be satisfied, but it is a realistic number since 
$N=1024$ yields a Doppler spacing of $\Delta \nu$ corresponding to $1.3 \mathrm{~Hz}$ at $1250 \mathrm{symb} / \mathrm{sec}$. With these approximations (4.105) yields

$$
\sum_{n=m}^{m+N-1} y(n) z^{*}(n-i) e^{-j 2 \pi p \Delta \nu n} \approx N U_{i, p}
$$

Therefore the ML estimate at delay $l_{i}$ is given approximately by choosing the largest peaks of the left hand side in (4.107). We note that this is carried out separately for each delay because $z(n)$ is white. A block diagram of the channel tracker given by (4.107) is shown in Fig. 4-21. In this figure we are looking at the delay $l_{0}=0$ and we assume that the number of coefficients at this delay is $K$. The frequency tap spacing

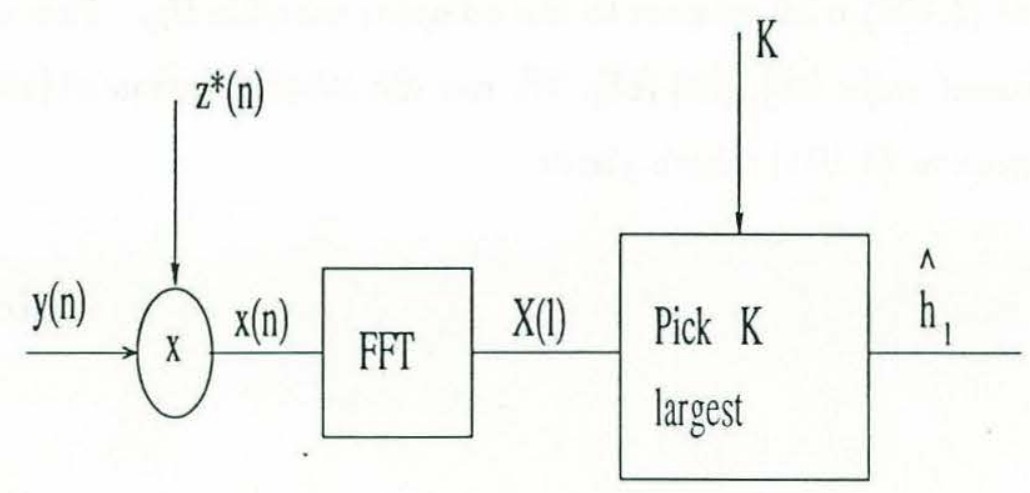

Figure 4-21: The channel tracking of a doubly spread channel implemented with block processing.

$\Delta \nu$ is chosen based on the Doppler spread, and it is discussed in Section 4.3.6. Thus it may not be possible to satisfy $\Delta \nu=1 / N$. Moreover, as is shown in Section 4.3.8, the stability of the receiver in (4.105) and also its recursive counterpart in (4.109) is limited by $\Delta \nu$. If $\Delta \nu$ is too small the matrix in (4.106) approaches a singular matrix, and we return to this case in Section 4.3.8. We also not that (4.107) is only valid for large $N$ so that the expression in the curly brackets of (4.106) approximates the true correlation closely. 


\section{Recursive processing}

The signal model (4.83) also allows for recursive estimation of the channel, and the TU-RLS algorithm can be used to track its coefficients $U_{l, k}(n)$. We assume the AR(1) model (4.86) for the channel coefficients, and thus the state space model (4.87) is valid. Specifically, if we consider the exponentially weighted version of (4.103) which is

$$
\begin{aligned}
J & =\sum_{m=0}^{n} \lambda^{n-m}|e(m)|^{2} \\
& =\sum_{m=0}^{n} \lambda^{n-m}\left|y(m)-\mathbf{c}^{H}(m) A^{m-n} \hat{\mathbf{h}}(n)\right|^{2}
\end{aligned}
$$

we have the cost function that is minimized by the TU-RLS algorithm. We define

$$
\begin{aligned}
& \mathbf{c}^{H}(n, l)=z(n-l)\left[\begin{array}{lll}
1 & \cdots & 1
\end{array}\right]^{T} \\
& \mathbf{c}^{H}(n)=\left[\mathbf{c}^{H}\left(n, l_{0}\right) \cdots \mathbf{c}^{H}\left(n, l_{L-1}\right)\right] \\
& \hat{\mathbf{h}}(n)=\left[\hat{U}_{l_{0}, k_{0}}(n) e^{j 2 \pi k_{0} \Delta \nu n} \cdots \hat{U}_{l_{L-1}, k_{L-1}}(n) e^{j 2 \pi k_{L-1} \Delta \nu n}\right]^{T}
\end{aligned}
$$

where the dimension of $\mathbf{c}(n, l)$ is $\operatorname{dim}(\mathbf{c}(n, l))=K_{l} \times 1$ and $K_{l}$ is the number of Fourier coefficients at lag $l$. The TU-RLS algorithm yields the recursion in (4.92), and a block diagram of the channel tracker given by (4.109) and (4.92) is shown in Fig. 4-22.

\section{Comparison of block and recursive estimates}

The recursive estimate (4.92) and (4.109) is related to the block estimate (4.107), and we now show this connection for the case of purely Doppler spread signal. In this case the $\mathrm{AR}(1)$ parameters are all identical and equal to

$$
\alpha=2-\cos \left(\omega_{0} / 2 L\right)-\sqrt{\cos ^{2}\left(\omega_{0} / 2 L\right)-4 \cos \left(\omega_{0} / 2 L\right)+3}
$$




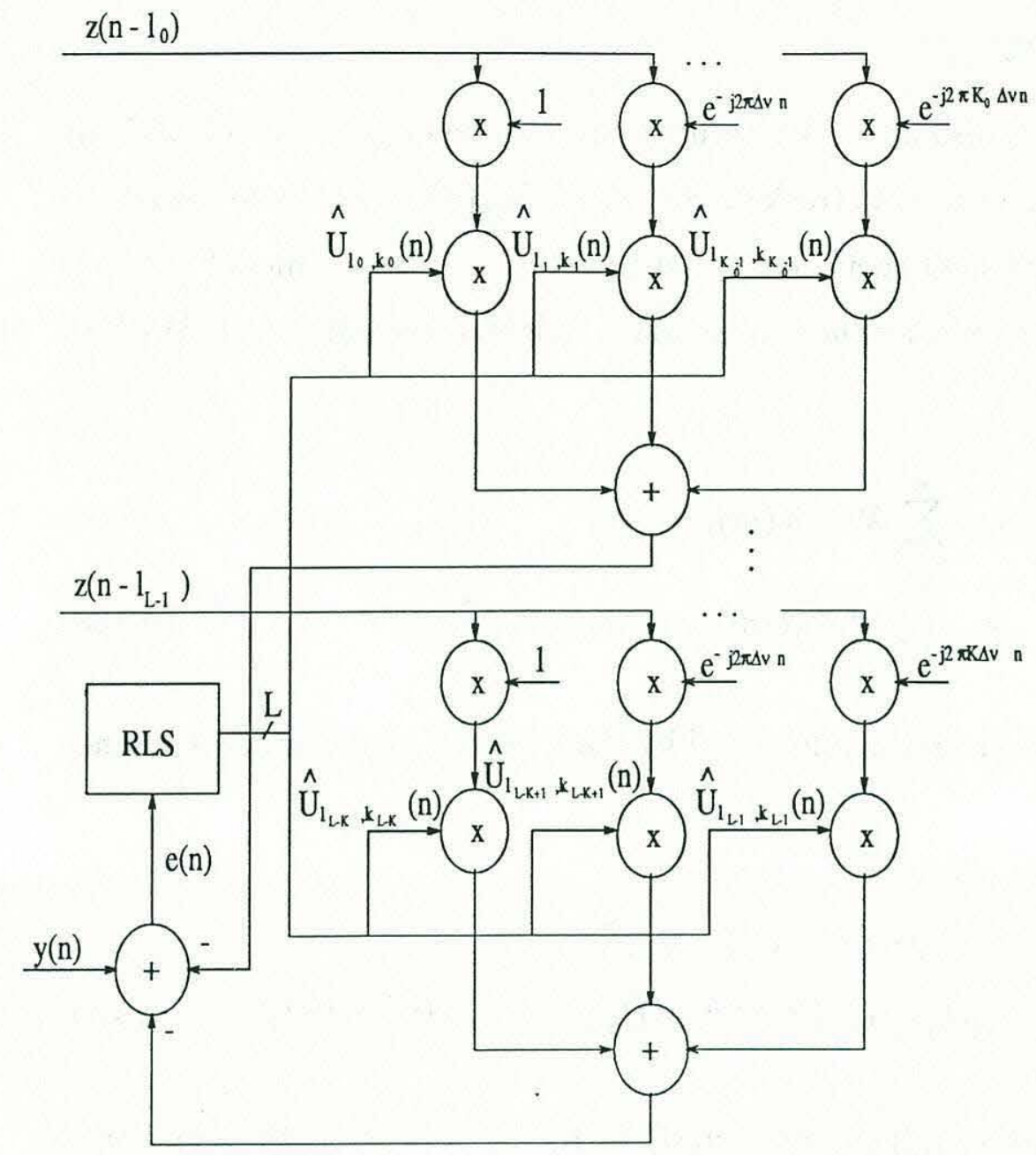

Figure 4-22: The channel tracking of a doubly spread channel implemented with recursive processing. There are 5 delay bins between the first energy cluster at 3 msec and the second cluster at 5 msec.

where $\omega_{0}=2 \pi B / f_{s}$ is the $3 \mathrm{~dB}$ bandwidth of the Doppler spread $B$ for a symbol rate of $f_{s}$. Each of the $L$ taps is now tracking a process of bandwidth $B / L$ corresponding to $\omega_{0} / 2 L$. The equation (4.110) is derived from the power spectrum of an $\operatorname{AR}(1)$ process in such a way that $\alpha$ for $L=1$ corresponds to the $3 \mathrm{~dB}$ bandwidth of the $\mathrm{AR}(1)$ process. Thus we have $L$ taps all at the same delay $l_{0}$ and we assume $l_{0}=0$ so that (4.109) yields

$$
\mathbf{c}^{H}(n)=z(n)\left[\begin{array}{lll}
1 & \cdots & 1
\end{array}\right]^{T}
$$




$$
\hat{\mathbf{h}}(n)=\left[\hat{U}_{0}(n) e^{j 2 \pi k_{0} \Delta \nu n} \cdots \hat{U}_{L-1}(n) e^{j 2 \pi k_{L-1} \Delta \nu n}\right]^{T}
$$

In this case we have a purely Doppler spread channel model. The solution to (4.92) and (4.111) is presented in Appendix B and given by

$$
\begin{aligned}
\hat{\mathbf{h}}(n) & =\left[\sum_{m=0}^{n} \lambda^{n-m}\left(A^{H}\right)^{m-n} \mathbf{c}(m) \mathbf{c}^{H}(m) A^{m-n}\right]^{-1} \sum_{m=0}^{n} \lambda^{n-m}\left(A^{H}\right)^{m-n} \mathbf{c}(m) y(m) \\
& =R_{c}^{-1} \sum_{m=0}^{n} \lambda^{n-m}\left(A^{H}\right)^{m-n} \mathbf{c}(m) y(m)
\end{aligned}
$$

provided that the inverse in this expression exists. By inserting $\mathbf{c}(n)$ from (4.111) in the matrix $R_{c}$ of (4.112) and using $A$ from (4.87) with $0 \leq k<L$ we get

$$
\begin{aligned}
R_{c} & =\sum_{m=0}^{n} \lambda^{n-m}\left(A^{H}\right)^{m-n} \mathbf{c}(m) \mathbf{c}^{H} A^{m-n}(m) \\
& =\sum_{m=0}^{n}\left(\lambda / \alpha^{2}\right)^{n-m}\left[\begin{array}{ccc}
1 & \cdots & e^{-j 2 \pi(L-1) \Delta \nu(n-m)} \\
\vdots & & \vdots \\
e^{j 2 \pi(L-1) \Delta \nu(n-m)} & \cdots & 1
\end{array}\right]
\end{aligned}
$$

We note that the condition number of $R_{c}$, defined by the ratio of the largest to the smallest eigenvalue, determines the numerical accuracy needed to compute $R_{c}^{-1}$ in (4.112). We return to this issue when the receiver stability is discussed in Section 4.3.8. We now look at the structure of $R_{c}$ in order to get an expression for $\hat{\mathbf{h}}(n)$ that can be compared to the block estimate (4.107). The diagonal terms of $R_{c}$ are all equal and given by

$$
\left[R_{c}\right]_{l l}=\sum_{m=0}^{n}\left(\lambda / \alpha^{2}\right)^{n-m}=\frac{1-\left(\lambda / \alpha^{2}\right)^{n+1}}{1-\left(\lambda / \alpha^{2}\right)} \approx \frac{1}{1-\left(\lambda / \alpha^{2}\right)}
$$

where the last approximation is good as $n$ gets large. We do not consider the range of $\alpha$ for which $\alpha^{2} \leq \lambda$ because this corresponds to a tracking bandwidth that is less than the bandwidth of the Doppler process we are tracking. In this case the receiver 
is unable to converge due to insufficient tracking bandwidth, and (4.112) is invalid. An off-diagonal term at location $(l, k)$ is given by

$$
\begin{aligned}
{\left[R_{c}\right]_{l k} } & =\sum_{m=0}^{n}\left(\lambda / \alpha^{2}\right)^{n-m} e^{j 2 \pi(k-l) \Delta \nu m} \\
& =\frac{\left(\lambda / \alpha^{2}\right)^{n+1}-e^{j 2 \pi(k-l) \Delta \nu(n+1)}}{\left(\lambda / \alpha^{2}\right)-e^{j 2 \pi(k-l) \Delta \nu}} \approx \frac{e^{j 2 \pi(k-l) \Delta \nu n}}{e^{j 2 \pi(k-l) \Delta \nu}-\left(\lambda / \alpha^{2}\right)}
\end{aligned}
$$

The magnitude of (4.115) is a monotonically decreasing function of $k-l$ and also of $\Delta \nu$ for the ranges of these variables stated below. Thus the first diagonal, where $k-l=1$, has the largest magnitude and $R_{c}$ is a banded matrix where the width of the only band which is around the main diagonal is given by $\left(\lambda / \alpha^{2}\right)$ and $\Delta \nu$. Each component in $\hat{\mathbf{h}}(n)$ of (4.112) is a linear combination of the components of the vector on the right hand side of this equation. The expression (4.115) means that $R_{c}$ has constant magnitude elements vs time $n$, therefore this is also true for $R_{c}^{-1}$. Thus the coefficients in the linear combination, given by $R_{c}^{-1}$, have approximately constant magnitude. The range of $k-l$ is bounded by $2 L \ll N$, and this is always satisfied for realistic $N$ and $L$. Thus $e^{j 2 \pi(k-l) \Delta \nu}$ in (4.115) never traverses more than a small fraction of a full rotation, and this enables us for the range of $\lambda$ and $\Delta \nu$

$$
\begin{aligned}
\lambda / \alpha^{2} & \geq 0.999 \\
\Delta \nu & <\frac{1}{2 \pi} \cos ^{-1} \frac{100\left(1-\lambda / \alpha^{2}\right)^{2}-1-\left(\lambda / \alpha^{2}\right)^{2}}{2 \lambda / \alpha^{2}}
\end{aligned}
$$

to write

$$
\max _{k \neq l}\left[\left|\left[R_{c}\right]_{l k}\right|\right] \leq 0.1\left|\left[R_{c}\right]_{l l}\right|
$$

Thus, for this range we neglect off-diagonal terms in $R_{c}$ so that (4.112) yields

$$
\hat{\mathrm{h}}(n) \approx\left(1-\left(\lambda / \alpha^{2}\right)\right) /\left(\lambda / \alpha^{2}\right) \sum_{m=0}^{n}\left(\lambda / \alpha^{2}\right)^{n-m} u(m)\left[1 \cdots e^{j 2 \pi(L-1) \Delta \nu m}\right]^{H}
$$


We have that $\lambda / \alpha^{2}=.999$ and $\Delta \nu=1.6 \times 10^{-3}$ satisfying (4.116) corresponds to $0.95 \mathrm{~Hz}$ at $600 \mathrm{symb} / \mathrm{sec}$. This suggests that the recursive estimate is approximately a windowed Fourier transform of $U(n)$. In comparison the block estimate (4.107) in the purely Doppler spread case is a sliding window Fourier transform of $U(n)$. In the recursive estimate we have an exponential window whereas the block estimate uses a rectangular window of length $N$. Thus if we replace the exponential weighting in (4.108) with a fixed length $N$ rectangular window, corresponding to a version of the RLS known as sliding window RLS [65], the terms in (4.115) are all zero for $\Delta \nu=1 / N$ and the expression for $\hat{\mathbf{h}}(n)$ corresponding to (4.118) is exact. The approximation in (4.118) gets poorer as $\lambda$ decreases, and in this case the recursive estimate is given by (4.112).

The accuracy with which $\mathrm{h}(n)$ is estimated by (4.112) depends partially on the condition number of $R_{c}$, and we discuss this in Section 4.3.8. We assume that $R_{c}$ is well conditioned so that $\hat{U}_{l, k}$ yields a good estimate of $U_{l, k}$. Having determined the receiver coefficients $\hat{U}_{l, k}$ we can use (4.83) for decoding, and we now derive two receivers making use of this knowledge.

\section{Decode using ML estimate}

Given the signal model of (4.83) and the estimate $\hat{\mathrm{h}}$ in (4.92) we now estimate the transmitted data sequence $z(n)$ by using the ML criterion. Thus we assume $y(n)$ and $U_{l, k}$ known, and the task is to find $z(n)$. By the Gaussian noise assumption the probability density of $\mathbf{y}$ conditioned on $\mathbf{z}$ is Gaussian and the ML estimate $\hat{\mathbf{z}}$ follows readily

$$
\begin{aligned}
\hat{\mathbf{z}} & =\min _{\mathrm{z}} \sum_{n=m}^{m+N-1}\left|y(n)-\sum_{l, k} z(n-l) U_{l, k} e^{j 2 \pi k \Delta \nu n}\right|^{2} \\
& =\min _{\mathrm{z}}\left[2 \operatorname{Re}\left[\sum_{n=m}^{m+N-1} \sum_{l, k} y^{*}(n) z(n-l) U_{l, k} e^{j 2 \pi k \Delta \nu n}\right]\right.
\end{aligned}
$$




$$
\left.-\sum_{n=m}^{m+N-1} \sum_{l, k} \sum_{i, p} z(n-l) z^{*}(n-i) U_{l, k} U_{i, p}^{*} e^{-j 2 \pi(k-p) \Delta \nu n}\right] .
$$

This is the MLSE receiver when the channel is modeled with the delay-Dopplerspread function. To further illustrate this receiver we now assume the simple case of no delay spread, and we recognize the ML estimate as an IFS Doppler line since we can write

$$
\hat{z}(n)=\frac{y(n)}{\sum_{k} U_{k} e^{j 2 \pi k \Delta \nu n}} .
$$

There is nothing that prevents the denominator of (4.120) from going arbitrarily close to zero. This would result in noise enhancement, and it sometimes limits the usefulness of this receiver. This is avoided by adding to the denominator of $(4.120)$ a small constant term so that it never goes to zero, but this makes the estimate $\hat{z}(n)$ biased. The fundamental problem is the channel fade manifested by the gain $U(n)$ going to zero, and two ways to solve this is by means of diversity or coding.

It is instructive to use the concept of time-frequency duality developed elsewhere to proceed with the receiver discussion. It is clear that the dual of (4.120) is the zero forcing equalizer [82], and it is well known that this equalizer, which is an IIR filter, performs poorly for channels containing spectral nulls. The dual of a spectral null is a (time) fade, and this is the problem causing noise enhancement in (4.120). One classical way to circumvent the problem of the zero forcing equalizer is to constrain the equalizer structure to be an FIR filter. The problem of a channel fade is not alleviated by this approach, but the noise enhancement due to the IIR filter is eliminated. The dual of the FIR filter is the FFS Doppler line, and this motivates the following derivation of an FFS Doppler line based receiver. 


\section{Decode using an FFS Doppler line}

We now impose the constraint that we want to determine an estimate $\hat{z}(n)$ by using FFS Doppler lines at each delay as shown in Fig. 4-23. We use a slightly different channel model (4.121) and before we derive the receiver this change is motivated.

In the channel tracker we save many degrees of freedom by tracking nonzero taps only, and this is captured mathematically by not restricting the index $l$ in $z(n-l)$ from (4.83) to be consecutive numbers. We define the receiver coefficients $\mathbf{V}$ by means of (4.123) and (4.122). As will be shown below the solution for $\mathbf{V}$ involves a tensor which degenerates to matrices in the special cases of pure delay spread and pure Doppler spread. For example, in the case of pure delay spread the solution for $\mathbf{V}$ is given by the Wiener-Hopf equation which is solved by inverting a correlation matrix. When the channel response is sparse, as indicated by the values that $l$ take on in (4.83), the correlation matrix is banded but the inverse of a banded matrix is in general dense. In the decoder, where we need the inverse matrix, there is no gain in keeping the banded formulation. It gives more complex notation, therefore we modify the model $(4.83)$ by restraining the sequence $(l, k)$. In $(4.83)$ the $L$ pairs $(l, k)$ are arbitrary points. Now we consider $(l, k)$ to take on all integer values between $(0,0)$ and $(L-1, K-1)$. Therefore we write

$$
\begin{aligned}
y(n) & =\sum_{l=0}^{L-1} \sum_{k=0}^{K-1} z(n-l) U_{l, k} e^{j 2 \pi k \Delta \nu n}+w(n) \\
\hat{z}(n) & =\sum_{l=-L_{1} / 2}^{L_{1} / 2-1} \sum_{k=-K_{1} / 2}^{K_{1} / 2-1} V_{l, k} y(n-l) e^{j 2 \pi k \Delta \nu n} .
\end{aligned}
$$

The lower bound $(0,0)$ on $(l, k)$ is merely a notational convenience, and negative Doppler frequencies are easily accommodated by modifying this. The criterion to determine the coefficients $\mathbf{V}$ is the MMSE which is

$$
\mathbf{V}=\min _{\mathbf{V}} E\left[|\mathbf{z}(m)-\hat{\mathbf{z}}(m)|^{2}\right]
$$




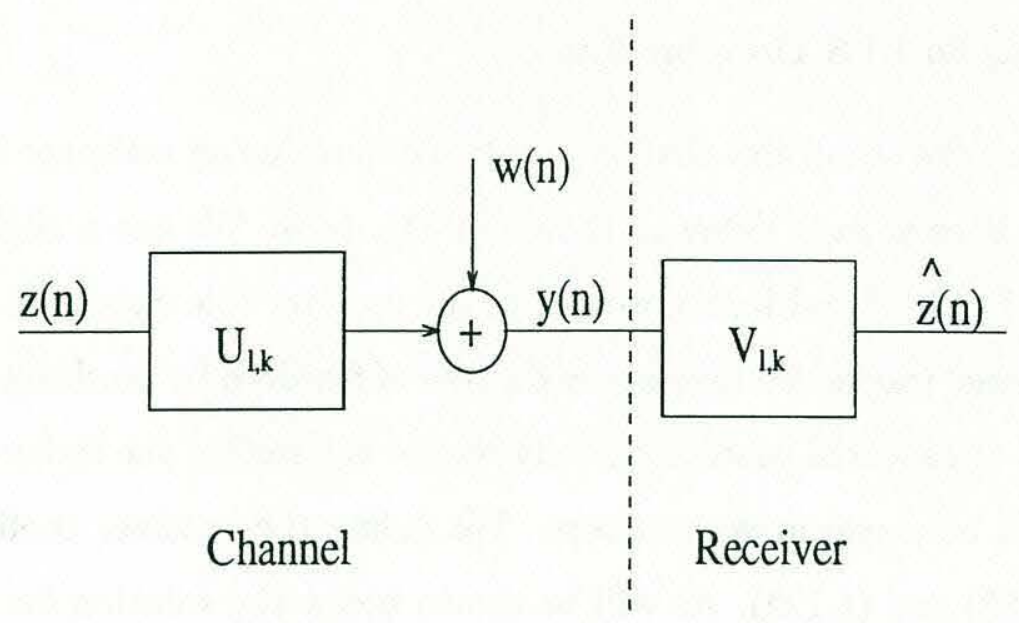

Figure 4-23: Decoding using FFS Doppler lines.

where

$$
\begin{aligned}
\mathbf{z}(m) & =[z(m) \cdots z(m+N-1)]^{T} \\
\hat{\mathbf{z}}(m) & =[\hat{z}(m) \cdots \hat{z}(m+N-1)]^{T} \\
\mathbf{V} & =\left[V_{-L_{1} / 2,-K_{1} / 2} \cdots V_{L_{1} / 2, K_{1} / 2}\right]^{T} .
\end{aligned}
$$

Thus we consider $U_{l, k}$ given, and we determine $\mathrm{V}$ by inserting $\hat{z}(n)$ from (4.121) in (4.122) and carry out the minimization by taking the derivative with respect to $V_{i, p}$. In this way $\mathbf{V}$ is determined, and the estimate $\hat{z}(n)$ is given by (4.122).

We model $z(n)$ and $w(n)$ as jointly WSS white random processes, and in order to find the minimum in (4.122) we solve $\partial E[\cdot] / \partial V_{i, p}=0$ which yields

$$
\sum_{n=m}^{m+N-1} E\left[z(n) y^{*}(n-i) e^{-j 2 \pi p \Delta \nu n}\right]=\sum_{\substack{n=m \\ j 2 \pi(k-p) \Delta \nu n}}^{m+N-1} E\left[\sum_{\substack{l=-L_{1} / 2 \\ e^{j}}}^{L_{1} / 2-1} \sum_{k=-K_{1} / 2}^{K_{1} / 2-1} V_{l, k} y(n-l) y^{*}(n-i) \times\right.
$$

where

$$
-L_{1} / 2 \leq i \leq L_{1} / 2-1
$$




$$
-K_{1} / 2 \leq p \leq K_{1} / 2-1 .
$$

By inserting $y(n)$ from (4.121) in (4.124), using $\Delta \nu=1 / N$ and carrying out the expectation and summation over $N$ we get

$$
\begin{aligned}
U_{-i,-p}^{*} & =\sum_{l=-L_{1} / 2}^{L_{1} / 2-1} \sum_{k=-K_{1} / 2}^{K_{1} / 2-1} V_{l, k}\left[\sum_{q=0}^{L-1} \sum_{r=0}^{K-1} U_{q, r} U_{q+l-i, r+k-p}^{*} e^{-j 2 \pi \Delta \nu[(r+k-p) i-r l]}\right. \\
& \left.+\sigma_{w}^{2} \delta(i-l) \delta(k-p)\right]
\end{aligned}
$$

where $\delta(k)$ is the Kronecker delta and

$$
\begin{aligned}
|l-i| & <L \\
|k-p| & <K .
\end{aligned}
$$

We have used that $z(n)$ is white and that

$$
\sum_{n=0}^{N-1} e^{j 2 \pi k n / N}=\left\{\begin{array}{cc}
N & k=0 \\
0 & 0<k \leq N
\end{array}\right.
$$

in order to evaluate (4.126). The integers $n, i, l, q$ are time indices and $k, p, r$ are frequency indices. In the case of pure delay spread where $K=K_{1}=1$ and $p=k=$ $r=0$ we have

$$
U_{-i}^{*}=\sum_{l=-L_{1} / 2}^{L_{1} / 2-1} V_{l}\left[\sum_{q=0}^{L-1} U_{q} U_{q+l-i}^{*}+\sigma_{w}^{2} \delta(i-l)\right]
$$

for

$$
\begin{array}{r}
-L_{1} / 2 \leq i \leq L_{1} / 2-1 \\
|l-i|<L
\end{array}
$$


which corresponds to the Wiener-Hopf equation. In the case of pure Doppler spread where $L=L_{1}=1$ and $i=l=0$ we have

$$
U_{-p}^{*}=\sum_{k=-K_{1} / 2}^{K_{1} / 2-1} V_{k}\left[\sum_{r=0}^{K-1} U_{r} U_{r+k-p}^{*}+\sigma_{w}^{2} \delta(k-p)\right]
$$

for

$$
\begin{array}{r}
-K_{1} / 2 \leq p \leq K_{1} / 2-1 \\
|k-p|<K
\end{array}
$$

which corresponds to the dual of the Wiener-Hopf equation. In (4.129) we have suppressed the frequency index of $U, V$ which in this case is zero, and in (4.131) we have suppressed the time index of $U, V$ which in this case is zero. The receiver in (4.126) is suboptimal in the sense that it fails to minimize the error probability, but the disadvantage of the noise enhancement problem of the IFS Doppler line is absent. In evaluating $\mathbf{V}$ from the equations (4.126), (4.129) and (4.131) we assume that the channel $U_{l, k}$ is known, so that in practice we use $\hat{U}_{l, k}$ obtained from the channel tracker in these equations. We have assumed the Doppler spacing $\Delta \nu=1 / N$ in the derivations of this section, and as pointed out earlier this is a realistic spacing. If this spacing is not used the equations (4.129) and (4.131) yields only approximate MMSE solutions. In order to summarize the receiver structure and further illustrate Fig. 4-20 we show in Fig. 4-24 a more detailed picture of the receiver in the case of the recursive channel tracker and the FFS based decoder. The box marker "inversion" in Fig. 4-24 performs the operation of (4.126).

We are particularly interested in Doppler spread channels and their corresponding receivers. In this case the FFS based receiver is given by (4.131), and in Fig. 4-25 we show a detailed picture of the general receiver in Fig. 4-20 for the special case of a channel tracker for a purely Doppler spread channel and an FFS based decoder. 


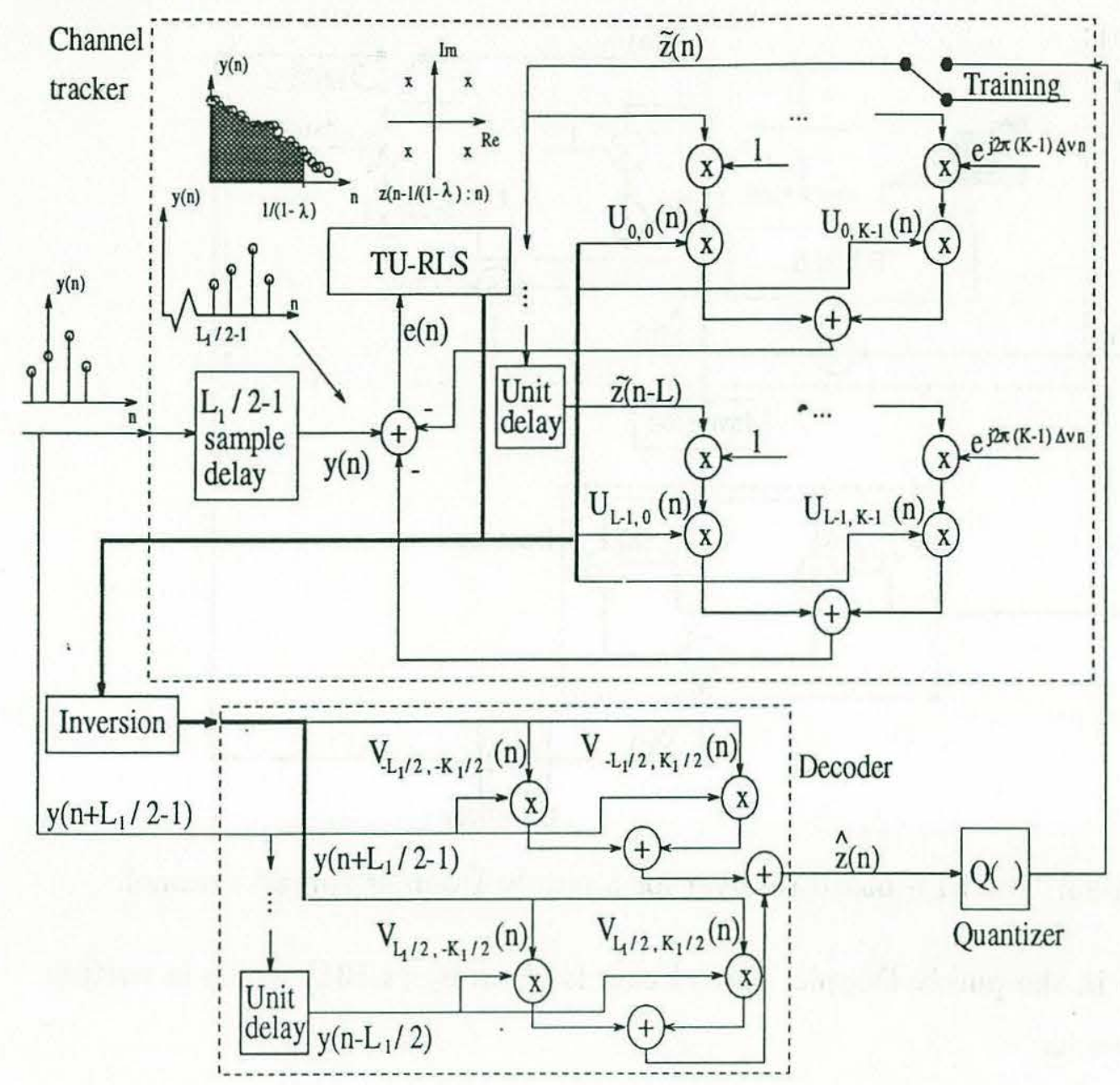

Figure 4-24: The FFS based receiver for a doubly spread channel.

\section{Constrained optimization}

We find in Section 4.4 that the unconstrained least squares solution (4.131) may give a Doppler line with excessive degrees of freedom exhibiting a behavior reminiscent of super directivity [67]. This is avoided by introducing a constrained solution to (4.122) which we now present in the case of purely Doppler spread channel. We use the constraint of unity white noise gain on the Doppler line coefficients which yields

$$
|\mathbf{V}|^{2}=1 \text {. }
$$




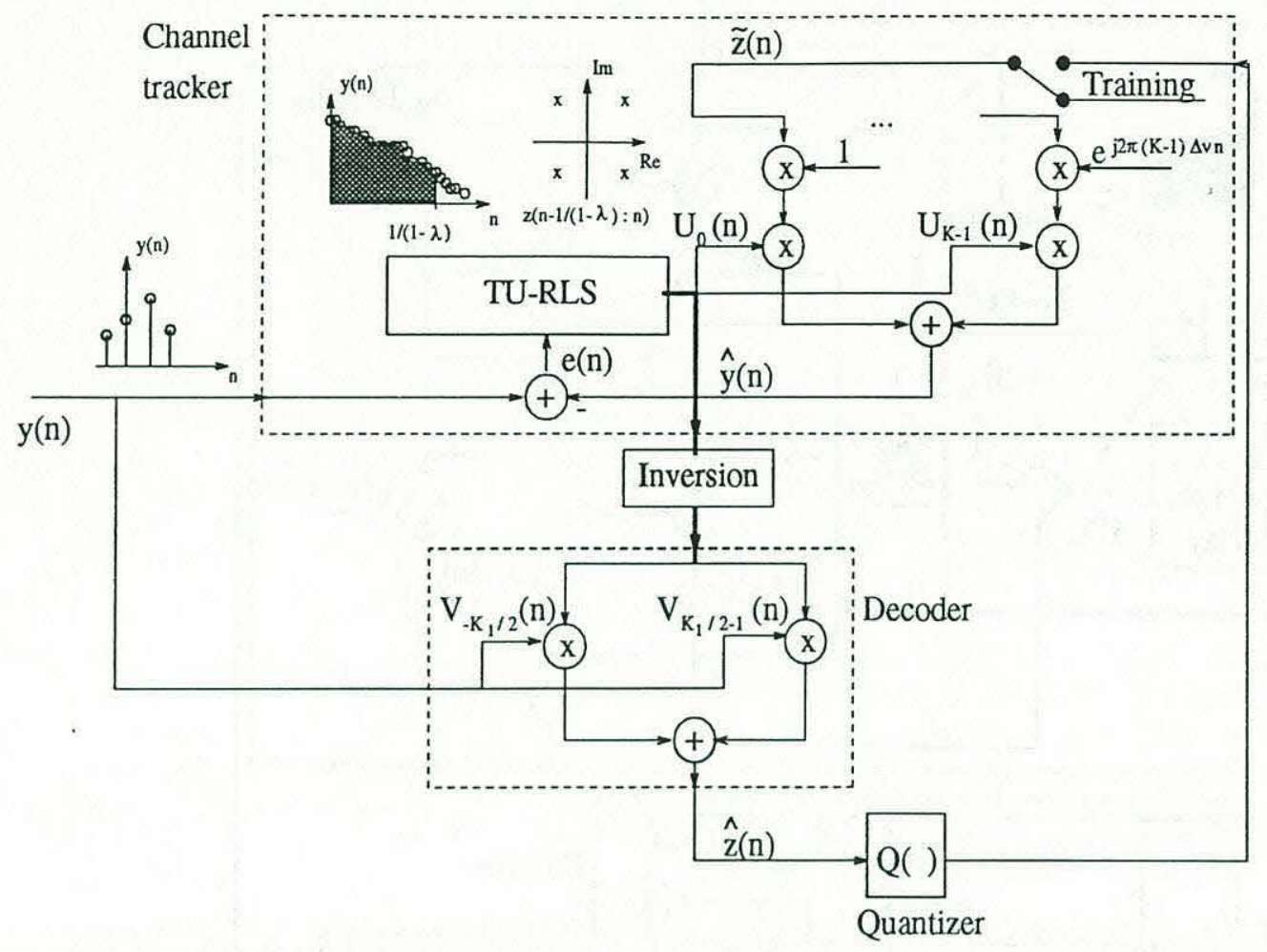

Figure 4-25: The FFS based receiver for a purely Doppler spread channel.

The solution in the purely Doppler spread case is given by (4.131) which is written in matrix form as

$$
S \mathrm{~V}=\mathrm{s}
$$

In order to find the constrained Doppler line we define the modified cost function

$$
J=E\left[|\mathbf{z}-\hat{\mathbf{z}}|^{2}\right]+\rho\left(|\mathbf{V}|^{2}-1\right)
$$

where $\rho$ is the Lagrange multiplier. The minimization of (4.135) over $\mathbf{V}$ yields

$$
\mathbf{V}=(S-\rho I)^{-1} \mathbf{s}
$$


where $\rho$ satisfies

$$
\mathbf{s}^{H}(S-\rho I)^{-H}(S-\rho I)^{-1} \mathbf{s}=1 .
$$

We solve (4.137) numerically for $\rho$ and insert this in (4.136) when we present an example of the constrained Doppler line in Section 4.4.

Summary The receiver tracking bandwidth at a single delay can be increased by employing Doppler lines provided that the physical interpretation of the delayDoppler spread function is changed. The delay-Doppler-spread function $U_{l, k}(n)$ introduced in (4.83) is interpreted as the scattering amplitude at $(l, k)$ whereas the recursive channel tracker (4.112) is approximately a sliding window Fourier-transform. This means that the tracked coefficients are the contribution to the delay-Dopplerspread function within a frequency band given by the Fourier-transform resolution. Thus the physical interpretation is that the channel tracker determines the Fourier coefficients of the time-varying scattering amplitude rather than the scattering amplitude directly. The receiver structure performs channel tracking and symbol decoding in parallel. The delay-Doppler-spread function is estimated using the channel input and output data sequences. The ML estimate is approximately found by performing a demodulation and Fourier transform of the received signal as given by (4.107) and shown in Fig. 4-21 where the demodulation is with respect to the transmitted data. A recursive estimate is given in (4.109) and (4.93), and the structure of this estimator is shown in Fig. 4-22. This estimate is shown to be approximately a sliding window Fourier-transform in (4.118), and this approximation is good for the range of $\lambda, \alpha$ and $\Delta \nu$ given in (4.116). This also relates the block estimate (4.107) and the recursive estimate (4.109): The block estimate is a Fourier-transform with a rectangular window that is moved so that blocks are non-overlapping. The recursive estimate is a Fourier-transform with an exponential window that is moved only one sample for each update. This is discussed in the comments to the equations (4.112) 
and (4.118). Based on the channel estimate two receivers are presented in (4.119) and (4.126). The first receiver is derived from the ML criterion of decoding assuming that the channel is known. In the case of pure Doppler spread this receiver reduces to an IFS Doppler line in (4.120).The second receiver is derived from the MMSE criterion and its structure is constrained to only contain FFS Doppler lines. In the case of a purely Doppler spread channel this receiver reduces to an FFS Doppler line in (4.131) with a constrained version in (4.136).

Decoders in the case of purely Doppler spread channel are suggested in (4.120) and (4.131). We further characterize the decoder part of our receiver in the general case by assuming that the TU-RLS is tracking the channel so that it delivers an

$\hat{\mathrm{h}}(n)$ that is close to $\mathbf{h}(n)$, and we turn to the problem of how to use this knowledge optimally to recover the transmitted data sequence. This is the task of the signal combiner in our receiver.

\subsubsection{Signal combiner}

The coherent combiner denoted "MMSE FIR" ("minimum mean square error finite impulse response") in Fig. 4-20 is now given as the Wiener filter $\hat{\mathrm{h}}_{0}(n)$ based on the estimated channel response $\hat{\mathrm{h}}(n)$, and we denote the m'th component of this vector $\hat{h}(n, m)$. Thus the equations to obtain the time-variant impulse response for the signal combiner, referring to Fig. 4-20, are

$$
\begin{aligned}
& y(n)=\sum_{m=0}^{L-1} \hat{h}(n, m) z\left(n-l_{m}\right)+w(n) \\
& \hat{z}(n)=\sum_{m=-L_{1} / 2}^{L_{1} / 2-1} \hat{h}_{0}(n, m) y(n-m)
\end{aligned}
$$

where $L$ is the length of $\hat{\mathrm{h}}(n)$ and $L_{1}$ is the number of taps in the signal combiner filter $\hat{h}_{0}(n, m)$. The time-variant impulse response $\hat{h}_{0}(n, m)$ of this filter is described 
by the Wiener-Hopf equation

$$
R_{y y}(n) \hat{\mathrm{h}}_{0}(n)=\mathrm{r}_{z y}(n) .
$$

The vector of values $y(n)$ implicitly used in forming $R_{y y}$ has length $L_{1}$ which is different from the length of $\mathbf{y}$ in (4.101). Note that we now consider $\hat{h}(n, m)$ given, i.e., we assume that the channel tracker is working properly, and $\hat{h}(n, m)$ is not a stochastic quantity in this context. The autocorrelation matrix $R_{y y}(n)$ and cross correlation vector $\mathbf{r}_{z y}(n)$ are expressed in terms of $\hat{h}(n, m)$ rather than as timeaveraged correlations:

$$
\begin{aligned}
{\left[R_{y y}(n)\right]_{m l} } & =\sum_{o=0}^{L-1} \hat{h}(n-l, o) \hat{h}^{*}(n-m, o+l-m)+\left[R_{w w}\right]_{m l} \\
r_{z y}^{m}(n) & =\hat{h}^{*}(n-m,-m)
\end{aligned}
$$

where $-L_{1} / 2 \leq m<L_{1} / 2,-L_{1} / 2 \leq l<L_{1} / 2$ and $r_{z y}^{m}(n)$ is element $m$ of $\mathrm{r}_{z y}(n)$. Note that this makes it necessary to estimate the noise covariance $R_{w w}$ explicitly, whereas when $R_{y y}$ and $\mathbf{r}_{z y}$ are estimated from received data the variance is implicitly estimated through the sample covariance of the received data. Also note that $R_{y y}$ is a non-Toeplitz matrix as opposed to its time-invariant counterpart. To see the structure of (4.139) better let us make an example. Consider the noise $w(n)$ to be white with variance $\sigma_{w}^{2}$ and the time-variant, normalized impulse response

$$
\begin{aligned}
& \hat{h}(n, m)=\left\{\begin{array}{cc}
\hat{h}_{0} e^{j 2 \pi \nu_{0} n} & m=0 \\
\hat{h}_{1} & m=1 \\
\hat{h}_{2} & m=2
\end{array}\right. \\
& \sum_{m}|\hat{h}(n, m)|^{2}=1 \forall n
\end{aligned}
$$

where $\nu_{0}=k_{0} \Delta \nu$. The physical interpretation of (4.141) may be a signal arriving over two direct ray paths $\hat{h}_{1}$ and $\hat{h}_{2}$ and one surface reflected ray path $\hat{h}_{0}$ with a long 
swell producing a Doppler shift $e^{j 2 \pi k_{0} \Delta \nu n}$. For $L_{1}=6$ (4.139) yields 


$$
\begin{aligned}
& {\left[\begin{array}{ccc}
1+\sigma_{w}^{2} & \hat{h}_{0} e^{j 2 \pi \nu_{0}(n-2)} \hat{h}_{1}^{*}+\hat{h}_{1} \hat{h}_{2}^{*} & \hat{h}_{0} e^{j 2 \pi \nu_{0}(n-1)} \hat{h}_{2}^{*} \\
\hat{h}_{0}^{*} e^{-j 2 \pi \nu_{0}(n-2)} \hat{h}_{1}+\hat{h}_{1}^{*} \hat{h}_{2} & 1+\sigma_{w}^{2} & \hat{h}_{0} e^{j 2 \pi \nu_{0}(n-1)} \hat{h}_{1}^{*}+\hat{h}_{1} \hat{h}_{2}^{*} \\
\hat{h}_{0}^{*} e^{-j 2 \pi \nu_{0}(n-1)} \hat{h}_{2} & \hat{h}_{0}^{*} e^{-j 2 \pi \nu_{0}(n-1)} \hat{h}_{1}+\hat{h}_{1}^{*} \hat{h}_{2} & 1+\sigma_{w}^{2} \\
0 & \hat{h}_{0}^{*} e^{-j 2 \pi \nu_{0} n} \hat{h}_{2} & \hat{h}_{0}^{*} e^{-j 2 \pi \nu_{0} n} \hat{h}_{1}+\hat{h}_{1}^{*} \hat{h}_{2} \\
0 & 0 & \hat{h}_{0}^{*} e^{-j 2 \pi \nu_{0}(n+1)} \hat{h}_{2} \\
0 & 0 & 0
\end{array}\right.}
\end{aligned}
$$

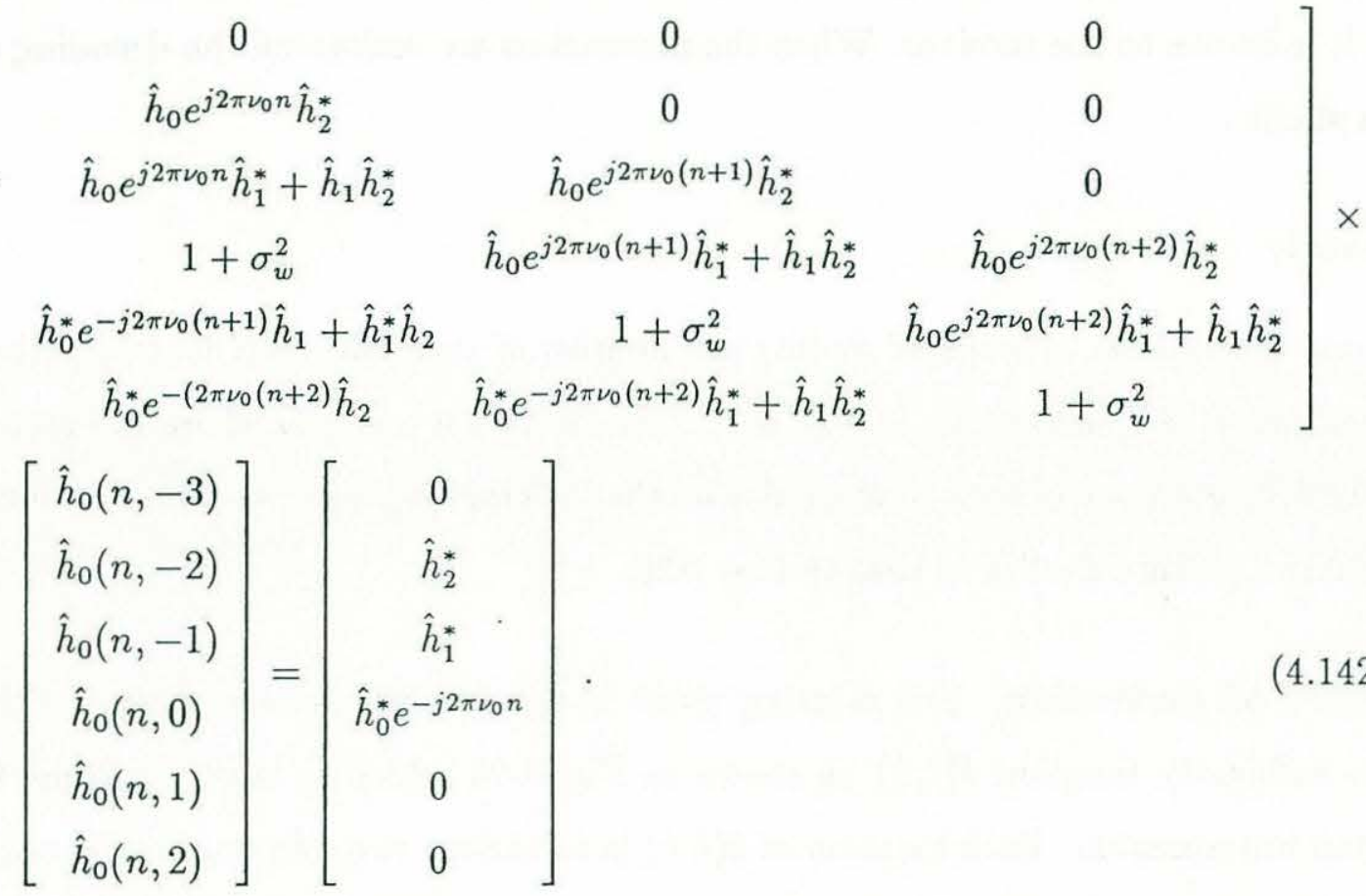

Thus the decoder is the solution to (4.139) which involves the inversion of the matrix $R_{y y}$ with the structure shown in (4.142). This yields the linear filter used in (4.138) to compute $\hat{z}(n)$, and it is updated based on the channel tracker output at each sample.

Summary The signal combiner is a FIR filter with its taps computed from the channel estimate by means of the Wiener-Hopf equation (4.139). We show an ex- 
ample of the structure of this matrix, which is updated every sample, in (4.142).

\subsubsection{Tap initialization}

Before the receiver can be used for decoding it needs to be initialized because the TU-RLS is based on knowledge of certain parameters. These are the positions of the signal returns in delay and Doppler, i.e., the pairs $(l, k)$ in $(4.83)$, and the number of taps $L$ that is used in the channel tracker. The estimation of these parameters is carried out by means of a training sequence that is transmitted before the data, and it is known to the receiver. When the parameters are estimated the decoding of data starts.

\section{Preview}

We now discuss the problem of finding the number of taps and their locations that is used in the channel tracker in Fig. 4-20. We consider a scenario where the crossambiguity function has been computed from the training sequence, and thus we have available a picture similar to that in Fig. 4-26.

Outline of derivation The starting point is the information contained in the cross-ambiguity function $\theta(l, k)$ as shown in Fig. 4-26 which is available prior to the tap initialization. Each location of $\theta(l, k)$ is a random variable due to the noise present in the received signal. The main idea is at each location $(l, k)$ to pose a classical detection problem [100]: A signal return is either present or absent (4.143), and the problem is to choose between including or omitting a tap (4.144) at the location in question. This procedure is repeated over all locations. The locations are ordered according to descending $\theta(l, k)$, so that the locations with large values are considered first. Thus we have a binary hypothesis test, and there are two possible errors in this assignment problem. They are shown in the section called "Energy losses" and the first is called "Omit tap when signal is present" corresponding to 


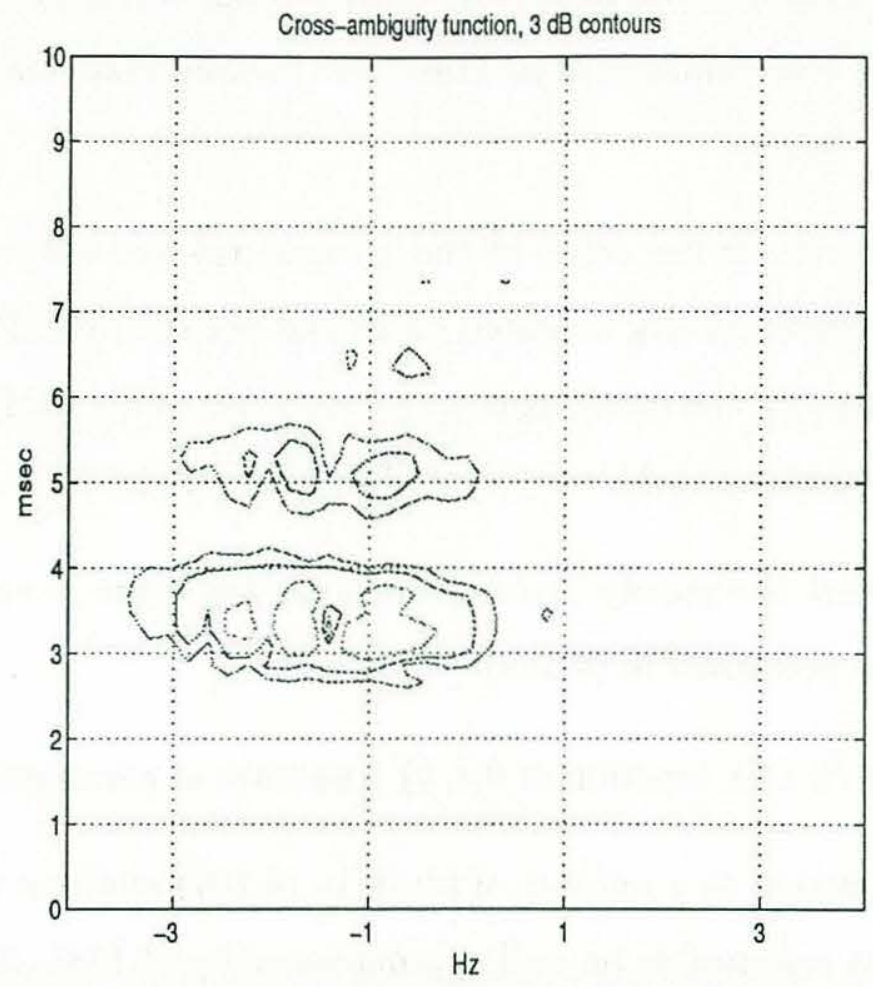

Figure 4-26: Contour plot of the cross-ambiguity function computed from the training sequence and the received data. There are 5 symbol intervals between the arrival clusters at 3 and $5 \mathrm{msec}$.

"Probability[Miss]" in detection theory. The second is called "Include tap when signal is absent" corresponding to "Probability[False alarm]" in detection theory. The energy losses play the role of the cost in the Bayesian framework we are using, and we minimize the expected cost. This is carried out in the section called "Tap selection rule". The rule makes use of the hypothesis probability $P_{0} . P_{0}$ is a function of the noise and signal level at each location of $\theta(l, k)$. In the section "The hypothesis probability" $P_{0}$ is expressed in terms of $\theta(l, k)$, and this completes the derivation. We show two examples in order to illustrate the rule.

Assumptions There are a number of assumptions involved in the derivation of the rule, and we list the most important here. 
- We order the locations where the binary hypothesis test is performed according to descending cross-ambiguity function. We assume that this is a good way to choose taps

- The cost criterion is the MSE of the channel tracker output. The channel tracker is an exponentially weighted LS fit and not the MMSE solution. When it operates properly the resulting error is very close to the MMSE performance, and we assume that it achieves this performance in (4.149)

- We assume that the steady state channel tracker error is as derived in Section 4.3 .8 and presented in (4.152)

- In expressing $P_{0}$ as a function of $\theta(l, k)$ a number of assumptions are made $\theta(l, k)$ is expressed as a number of terms in (4.164) and one term called $\theta_{1}$ in (4.167) is assumed to be well approximated by (4.168). This is motivated in Appendix C

Another term of $\theta(l, k)$ called $\theta_{2}$ in (4.175) is neglected, and this is also motivated in Appendix C

The time variation of $U_{l, k}(n)$ over $M$ is neglected in (4.169)

- We assume no apriori information about whether there is a signal present at any location

- We assume that a good estimate of the scattering strength is obtained from the mean of $\theta(l, k)$ in $(4.190)$

Steps The major steps of the derivation of the tap initialization rule and the corresponding equations are summarized in table 4.1.

The initialization of the taps is part of a system identification problem [64]. There is a fundamental issue that has implications for the way we propose to assign taps, 


\begin{tabular}{|l|l|}
\hline \hline Step & Equations \\
\hline Pose the binary hypothesis problem & $(4.143),(4.144)$ \\
Choose cost function & $(4.146),(4.149)$ \\
Compute cost of errors & $(4.155),(4.158)$ \\
Derive rule to minimize expected cost & $(4.161)$ \\
Express the hypothesis probability in terms of & \\
the cross-ambiguity function & $(4.165),(4.177),(4.178)$ \\
\hline \hline
\end{tabular}

Table 4.1: The major steps and the associated equations in the derivation of the tap initialization rule.

and we comment on this before proceeding with the assignment rule. As is shown in this chapter the rule we use for tap assignment is a function of how many taps that have already been assigned. The reason for this is that the noise level increases with the number of degrees of freedom in the channel tracker. Thus the order in which we assign taps is not arbitrary. Suppose in Fig. 4-26 we start assigning taps by considering, say, the lower left (delay,Doppler) location first. The result is different from the one obtained if we start with considering the largest signal returns around ( $3.5 \mathrm{msec},-2 \mathrm{~Hz}$ ). When we start assigning taps we have the cross-ambiguity function available, so we choose to consider the tap locations according to descending crossambiguity function amplitude. This prevents the situation described in the example above, and it makes the tap assignment algorithm unique. As we populate the channel tracker there is a tradeoff between including a weak signal return, increasing the number of degrees of freedom, and loosing a small portion of the transmitted signal energy by omitting the tap. The rule presented below takes care of this by not including taps when the associated increase in adaptation noise is larger than the signal energy at the location in question.

We now want to decide if a specific peak should be assigned to a tap. The tradeoff we have to make is the following: If we include a tap and the corresponding peak is noise the number of degrees of freedom increases and, as will be shown below, 
the noise level increases. If we omit a tap and the cross-ambiguity function peak corresponds to a signal return we loose some of the energy from the signal. Given a peak of a certain height there are two hypotheses:

$$
\begin{aligned}
& H_{0} \text { : The peak corresponds to a signal return } \\
& H_{1} \text { : The peak corresponds to noise }
\end{aligned}
$$

and we must decide between

$$
\begin{aligned}
& A_{1} \text { : include tap } \\
& A_{2} \text { : omit tap . }
\end{aligned}
$$

The task of the channel tracker in Fig. 4-20 is to estimate and track the channel impulse response $\mathbf{h}(n)$. A useful alternative interpretation of the channel tracker [105] is that of an optimal signal predictor in the MMSE sense. This means that the channel tracker attempts to minimize

$$
J=E\left[|y(n)-\hat{y}(n)|^{2}\right]
$$

where $\hat{y}(n)$ is the prediction of the received signal $y(n)$. We know that the MMSE is

$$
J=E\left[|y(n)|^{2}\right]-E\left[|\hat{y}(n)|^{2}\right]=\sigma_{y}^{2}-\sigma_{\hat{y}}^{2}
$$

where we have used that $E\left[y(n) \hat{y}^{*}(n)\right]=E\left[|\hat{y}(n)|^{2}\right]$ for the Wiener filter. Therefore in order to minimize $J$ we maximize $\sigma_{\hat{y}}^{2}$. We have the channel model (4.83) and if we have assigned taps at the positions $\left(l_{0}, k_{0}\right) \cdots\left(l_{L-2}, k_{L-2}\right)$ the TU-RLS obeys (4.87) and (4.92) which yields

$$
\hat{\mathrm{h}}(n+1)=A \hat{\mathrm{h}}(n)+\mathrm{k}(n) e(n)
$$




$$
\begin{aligned}
\hat{y}(n) & =\mathbf{c}^{H}(n) A \hat{\mathbf{h}}(n) \\
e(n) & =y(n)-\hat{y}(n) \\
\mathbf{c}^{H}(n) & =\sqrt{\mathcal{E}}\left[z\left(n-l_{0}\right) \cdots z\left(n-l_{L-2}\right)\right] \\
A & =\left[\begin{array}{ccc}
\alpha_{0} e^{j 2 \pi \nu_{0}} & 0 \\
& \ddots & \\
0 & & \alpha_{L-2} e^{j 2 \pi \nu_{L-2}}
\end{array}\right]
\end{aligned}
$$

where $\mathbf{c}^{H}(n)$ contains the transmitted sequence which is known since we are in training mode. We define the channel estimation error $\mathrm{h}_{e}$ by

$$
\mathbf{h}(n)=\hat{\mathbf{h}}(n)+\mathbf{h}_{e}(n)
$$

and note that when the channel tracker works properly it gives the estimate $\hat{\mathrm{h}}(n)$ close to the MMSE estimate, and we have approximately that

$$
E\left[\mathbf{h h}^{H}\right]=E\left[\hat{\mathbf{h}} \hat{h}^{H}\right]+E\left[\mathbf{h}_{e} \mathbf{h}_{e}^{H}\right]
$$

This is known as the principle of orthogonality in the Wiener filter literature [100]. The total variance of all components of the channel estimate is

$$
\begin{aligned}
\sigma_{\hat{h}}^{2} & =\operatorname{tr}\left(E\left[\hat{\mathbf{h}} \hat{h}^{H}\right]\right)=\operatorname{tr}\left(E\left[\mathbf{h h}^{H}\right]\right)-\operatorname{tr}\left(E\left[\mathbf{h}_{e} \mathbf{h}_{e}^{H}\right]\right) \\
& =\sigma_{h}^{2}(L-1)-\sigma_{h_{e}}^{2}(L-1)=\mathcal{E}_{\hat{h}}
\end{aligned}
$$

and we assign taps by maximizing this quantity. We see the relation to (4.146) by means of (4.147), and we choose this criterion because it yields a simple rule for tap assignment. The signal model given by (4.83) can be written

$$
\begin{aligned}
y(n) & =\sqrt{\mathcal{E}} \sum_{(l, k) \neq\left(l_{L-1}, k_{L-1}\right)} z(n-l) U_{l, k}(n) e^{j 2 \pi k \Delta \nu n} \\
& +z\left(n-l_{L-1}\right) U_{l_{L-1}, k_{L-1}}(n) e^{j 2 \pi k_{L-1} \Delta \nu n}+w(n)
\end{aligned}
$$


where we have written explicitly the signal contribution at location $\left(l_{L-1}, k_{L-1}\right)$ and $U_{l, k}(n)$ is an $\mathrm{AR}(1)$ process given by (4.86) with variance given by (4.88). We consider a scenario where $L-1$ taps have been assigned to the channel tracker so that $\mathbf{h}$ has dimension $L-1 \times 1$. The channel estimation error is derived in Section 4.3.8. It is given by (4.221) which for this case yields

$$
E\left[\mathbf{h}_{e} \mathbf{h}_{e}^{H}\right]=\left[I-\tilde{P} Q_{0}\right] A E\left[\mathbf{h}_{e} \mathbf{h}_{e}^{H}\right] A^{H}\left[I-\tilde{P} Q_{0}\right]^{H}+\tilde{P} Q_{0} \tilde{P}^{H} \sigma_{w}^{2}+R
$$

where from (4.88)

$$
E\left[\mathbf{v v}^{H}\right]=R=\left[\begin{array}{ccc}
\left(1-\alpha_{0}^{2}\right) u_{0}^{2} & & 0 \\
& \ddots & \\
0 & & \left(1-\alpha_{L-2}^{2}\right) u_{L-2}^{2}
\end{array}\right]
$$

$Q_{0}$ is the covariance matrix of $\mathbf{c}(n)$ and $\tilde{P}$ is the matrix given by (B.7) in Appendix B. Note that $E\left[h_{e} h_{e}^{H}\right]$ occurs on both sides of (4.152) because we are looking for the steady state solution of the difference equation (4.221). We find $E\left[\mathbf{h}_{e} \mathbf{h}_{e}^{H}\right]$ by solving (4.152) numerically. The reader is referred to the discussion pertaining to (4.221) for a derivation of (4.152). We now want to decide if a tap should be included at location $\left(l_{L-1}, k_{L-1}\right)$.

\section{Energy losses}

There are two ways of making an error, and each has an associated energy loss. Both these errors give a decrease in the energy of $\hat{\mathrm{h}}(n)$ and thus $\sigma_{\hat{h}}^{2}$ is a function of the choice we make. The energy $\sigma_{\tilde{h}}^{2}=\mathcal{E}_{\hat{h}}$ before we process the $L$ 'th tap is given by (4.150) and we now proceed with quantifying the energy loss in each case. 
Omit tap when signal is present We now consider the scenario under $H_{0}$ where the tap in question corresponds to a signal return. We can write the energy of $\hat{\mathbf{h}}(n)$ in each of the two cases

$$
\begin{aligned}
& A_{1}: \sigma_{\hat{h}}^{2}=\sigma_{h}^{2}(L)-\sigma_{h_{e}}^{2}(L) \\
& A_{2}: \sigma_{\hat{h}}^{2}=\sigma_{h}^{2}(L-1)-\sigma_{h_{e}}^{2}(L-1)=\mathcal{E}_{\hat{h}} .
\end{aligned}
$$

The energy loss is the difference between $A_{1}$ and $A_{2}$ which yields

$$
\Delta_{0}=u_{L-1}^{2}-\sigma_{h_{e}}^{2}(L)+\sigma_{h_{e}}^{2}(L-1)
$$

where we use

$$
\sigma_{h}^{2}(L)=\sum_{i=0}^{L-1} u_{i}^{2} .
$$

The quantity $\Delta_{0}$ is the loss if we erroneously choose $A_{2}$. We note that $\Delta_{0}$ may take on both positive and negative values, and this partly depends on the signal strength $u_{i}^{2}$. If the signal component is strong, corresponding to large $u_{i}^{2}$, it is more likely that $\Delta_{0}$ is positive, and then the gain is positive when we choose $A_{1}$. However, for a weak signal the increase in signal energy gained by including a tap may be canceled by the increased adaptation noise from including another tap. This is captured by $\Delta_{0}<0$ for this case. Therefore the rule to be presented, based on $\Delta_{0}$, implies that weak signal components are omitted depending on their strength relative to the increase in adaptation noise.

Include tap when signal is absent We now consider the other error which is to include a tap at a location where the signal is absent. In this case we make an error 
if we include a tap at the location in question, and we get the energy in $\hat{\mathbf{h}}(n)$ as

$$
\begin{aligned}
& A_{1}: \sigma_{\hat{h}}^{2}=\sigma_{h}^{2}(L-1)-\sigma_{h_{e}}^{2}(L) \\
& A_{2}: \sigma_{\hat{y}}^{2}=\sigma_{h}^{2}(L-1)-\sigma_{h_{e}}^{2}(L-1)=\mathcal{E}_{\hat{h}} .
\end{aligned}
$$

This gives the loss

$$
\Delta_{1}=\sigma_{h_{e}}^{2}(L)-\sigma_{h_{e}}^{2}(L-1)
$$

if we erroneously choose $A_{1}$. We now have quantified the energy losses associated with each error, and they will be used in the following when a rule for tap selection is derived.

\section{Tap selection rule}

We have no knowledge which of the two hypotheses $H_{0}$ or $H_{1}$ that is true, but if we find their probabilities of occurrence a selection rule based on the maximization of the expected energy can be found. Table 4.2 shows the value of the energy that results under each of the hypotheses. We now need the probabilities of occurrence

\begin{tabular}{|l|l|l|}
\hline \hline & $H_{0}$ & $H_{1}$ \\
\hline$A_{1}:$ include tap & $\mathcal{E}_{\hat{h}}+\Delta_{0}$ & $\mathcal{E}_{\hat{h}}-\Delta_{1}$ \\
$A_{2}:$ omit tap & $\mathcal{E}_{\hat{h}}$ & $\mathcal{E}_{\hat{h}}$ \\
\hline \hline
\end{tabular}

Table 4.2: The energy in $\hat{h}(n)$ for different cases of tap selection

for the two hypotheses, and these probabilities are

$$
\begin{aligned}
& \operatorname{Pr}\left[H_{0} \mid \theta\left(l_{L-1}, k_{L-1}\right)>\theta_{t}\right]=P_{0}=\operatorname{Pr}[\text { "Peak is signal" } \mid \text { Peak height }] \\
& \operatorname{Pr}\left[H_{1} \mid \theta\left(l_{L-1}, k_{L-1}\right)>\theta_{t}\right]=1-P_{0}
\end{aligned}
$$


where $\theta_{t}$ is the measured value of the cross-ambiguity function $\theta\left(l_{L-1}, k_{L-1}\right)$. From the table we see that the expected energy for each of the choices $A$ or $B$ is

$$
\begin{aligned}
& E\left[\sigma_{\hat{h}}^{2} \mid A_{1}\right]=\mathcal{E}_{\hat{h}}+P_{0} \Delta_{0}-\Delta_{1}+P_{0} \Delta_{1} \\
& E\left[\sigma_{\hat{h}}^{2} \mid A_{2}\right]=\mathcal{E}_{\hat{h}} .
\end{aligned}
$$

Thus the rule to maximize the expected energy is

$$
\begin{aligned}
& P_{0}\left(\Delta_{0}+\Delta_{1}\right)-\Delta_{1}>0: \text { Choose } A_{1} \\
& P_{0}\left(\Delta_{0}+\Delta_{1}\right)-\Delta_{1}<0: \text { Choose } A_{2}
\end{aligned}
$$

We note that $\sigma_{h_{e}}^{2}(L)$ is a function of the frequency tap spacing $\Delta \nu$ because it enters through $\tilde{P}$ as shown in (4.113) and (B.7). When the Doppler spread $B$ at a certain delay is given we choose

$$
\Delta \nu=\frac{B}{L f_{s}}
$$

where $f_{s}$ is the symbol rate. The implication of this is that when we add a tap, in addition to the increase in $\sigma_{h_{e}}^{2}(L)$ due to increased self noise and lag noise of the channel tracker, we get an increase in $\sigma_{h_{e}}^{2}(L)$ due to an increasing condition

number of $\tilde{P}$ because $\Delta \nu=0$ renders $\tilde{P}$ singular as seen from (4.113). We show in Section 4.3 .8 that this limits the stability of the receiver.

\section{The hypothesis probability}

The rule in (4.161) makes use of $P_{0}$, and we now find an expression for this probability. The cross-ambiguity function from Section 2.3 is given by

$$
\theta(n, k)=\mathcal{E}\left|\sum_{m=0}^{M-1} z(m) y^{*}(m-n) e^{j 2 \pi k \Delta \nu m}\right|^{2}
$$


The signal model under $H_{0}$ is given by (4.151) and by using this we find the crossambiguity function as

$$
\begin{aligned}
\theta(n, k)= & \mathcal{E}^{2}\left|\sum_{(l, o)} \sum_{m=0}^{M-1} z(m) z^{*}(m-n-l) U_{l, o}^{*}(m-n) e^{-j 2 \pi \Delta \nu[o(m-n)-k m]}\right|^{2} \\
+ & 2 \mathcal{E}^{3 / 2} \operatorname{Re}\left[\sum_{(l, o)} \sum_{m=0}^{M-1} z(m) z^{*}(m-n-l) U_{l, o}^{*}(m-n) e^{-j 2 \pi \Delta \nu[o(m-n)-k m]} \times\right. \\
& \left.\sum_{m=0}^{M-1} z(m) w^{*}(m-n) e^{j 2 \pi k \Delta \nu m}\right] \\
+ & \mathcal{E}\left|\sum_{m=0}^{M-1} z(m) w^{*}(m-n) e^{j 2 \pi k \Delta \nu m}\right|^{2} .
\end{aligned}
$$

We now without loss of generality assume that $\left(l_{L-1}, k_{L-1}\right)=(0,0)$, and this yields

$$
\begin{aligned}
\theta(0,0)= & \mathcal{E}^{2}\left|\sum_{(l, o)} \sum_{m=0}^{M-1} z(m) z^{*}(m-l) U_{l, 0}^{*}(m) e^{-j 2 \pi o \Delta \nu m}\right|^{2} \\
+ & 2 \mathcal{E}^{3 / 2} \operatorname{Re}\left[\sum_{(l, o)} \sum_{m=0}^{M-1} z(m) z^{*}(m-l) U_{l, 0}^{*}(m) e^{-j 2 \pi o \Delta \nu m} \times\right. \\
& \left.\sum_{m=0}^{M-1} z(m) w^{*}(m)\right] \\
+ & w_{\theta}(0,0)
\end{aligned}
$$

where

$$
w_{\theta}(n, k)=\mathcal{E}\left|\sum_{m=0}^{M-1} z(m) w^{*}(m-n) e^{j 2 \pi k \Delta \nu m}\right|^{2} .
$$

The first term of (4.165) is

$$
\theta_{1}=\mathcal{E}^{2}\left|\sum_{(l, o)} \sum_{m=0}^{M-1} z(m) z^{*}(m-l) U_{l, o}^{*}(m) e^{-j 2 \pi o \Delta \nu m}\right|^{2} .
$$


We show in Appendix $C$ that the approximation of (4.167) by

$$
\theta_{1}=\mathcal{E}^{2} \sum_{(l, o)}\left|\sum_{m=0}^{M-1} z(m) z^{*}(m-l) U_{l, o}^{*}(m) e^{-j 2 \pi \circ \Delta \nu m}\right|^{2}
$$

leaves the mean and variance of this term unchanged, and thus we use (4.168) for the first term of (4.165). $U_{l, k}(m)$ varies slowly and if it is assumed to be constant over $M$ we have

$$
\theta_{1}=\mathcal{E}^{2} \sum_{(l, o)}\left|U_{l, o}\right|^{2}\left|\sum_{m=0}^{M-1} z(m) z^{*}(m-l) e^{-j 2 \pi o \Delta \nu m}\right|^{2}
$$

We seek a simpler expression for $\theta_{1}$, and to obtain this we observe that the $L-1$ terms of (4.169) all contain the signal ambiguity function

$$
\theta_{0}(l,-o)=\mathcal{E}^{2}\left|\sum_{m=0}^{M-1} z(m) z^{*}(m-l) e^{-j 2 \pi o \Delta \nu m}\right|^{2}
$$

We have that [101]

$$
\begin{aligned}
\sum_{n=0}^{M-1} \sum_{k=0}^{M-1} \theta_{0}(n, k) & =M(M \mathcal{E})^{2} \\
\theta_{0}(0,0) & =(M \mathcal{E})^{2}
\end{aligned}
$$

where the first equation is the volume invariance property of the ambiguity-function, and the second equation follows from (4.163). It is desirable to have the level of $\theta_{0}(n, k)$ outside $\theta_{0}(0,0)$ as low as possible, and thus the best case scenario is when the rest of the volume of $\theta_{0}(n, k)$ is equally spread over $(n, k)$. The level $\Theta$ of $\theta_{0}(n, k)$ outside $(0,0)$ in this case is given by [101]

$$
\begin{aligned}
(M \mathcal{E})^{2}+\Theta\left(M^{2}-1\right) & =M(M \mathcal{E})^{2} \\
\Theta & \approx M \mathcal{E}^{2} .
\end{aligned}
$$


As noted in Section 2.3 we use unnormalized ambiguity functions, unlike [101] where $\theta_{0}(0,0)=1$. The difference is nothing but a multiplication by a constant, and it has no consequences for our derivation. Specifically, the distance from the peak to the noise floor of $\theta_{0}(l, k)$ as given by (4.172) is the same independent of normalization. The $\mathrm{m}$-sequence will determine $\theta_{0}(n, k)$, and numerical computations for $M$ in the range 64-1024 show that the peak level (worst case scenario) of $\theta_{0}(n, k)$ outside $(0,0)$ can be $4-5$ times $\Theta$ for ranges of $(n, k)$ corresponding to realistic delays and Doppler shifts. The distance to the peak $\theta_{0}(0,0)$ is roughly $M$, so the best case scenario is reasonable to use. Thus we approximate

$$
\theta_{0}(n, k) \approx \begin{cases}(M \mathcal{E})^{2} & (n, k)=(0,0) \\ M \mathcal{E}^{2} & (n, k) \neq(0,0)\end{cases}
$$

This property of the m-sequences when used in ambiguity functions is also suggested in [101], and by means of (4.173) we approximate (4.165)

$$
\begin{aligned}
\theta(0,0) \approx & \left|U_{0,0}\right|^{2}(M \mathcal{E})^{2}+\sum_{(l, o) \neq(0,0)}\left|U_{l, o}\right|^{2} M \mathcal{E}^{2} \\
+ & 2 \mathcal{E}^{3 / 2} \operatorname{Re}\left[\sum_{(l, o)} U_{l, o}^{*} \sum_{m=0}^{M-1} z(m) z^{*}(m-l) e^{-j 2 \pi o \Delta \nu m} \times\right. \\
& \left.\sum_{m=0}^{M-1} z(m) w^{*}(m)\right] \\
+ & w_{\theta}(0,0) .
\end{aligned}
$$

The third term of (4.174) is a product of two uncorrelated random processes and we define

$$
\begin{aligned}
\theta_{2}= & 2 \mathcal{E}^{3 / 2} \operatorname{Re}\left[\sum_{(l, o)} U_{l, o}^{*} \sum_{m=0}^{M-1} z(m) z^{*}(m-l) e^{-j 2 \pi o \Delta \nu m} \times\right. \\
& \left.\sum_{m=0}^{M-1} z(m) w^{*}(m)\right] .
\end{aligned}
$$


We show in Appendix $\mathrm{C}$ that $\theta_{2}$ is a zero-mean random variable with negligible impact on $\theta(0,0)$ of $(4.174)$ compared to $\theta_{1}$. Thus we omit this term and write $(4.174)$ as

$$
\theta(0,0)=\left|U_{0,0}\right|^{2}(M \mathcal{E})^{2}+\sum_{(l, o) \neq(0,0)}\left|U_{l, o}\right|^{2} M \mathcal{E}^{2}+w_{\theta}(0,0)
$$

The derivation in (4.165)-(4.176) is valid for the signal model (4.151) which is the model under hypothesis $H_{0}$. It is clear that under $H_{1}$ the only difference in (4.164) is that the term containing $U_{0,0}$ is omitted, therefore by looking at the specific location $(0,0)$ under the two hypotheses we have

$$
\theta(0,0)= \begin{cases}\left|U_{0,0}\right|^{2}(M \mathcal{E})^{2}+\sum_{(l, o) \neq(0,0)}\left|U_{l, o}\right|^{2} M \mathcal{E}^{2}+w_{\theta}(0,0) & H_{0} \\ \sum_{(l, o) \neq(0,0)}\left|U_{l, o}\right|^{2} M \mathcal{E}^{2}+w_{\theta}(0,0) & H_{1}\end{cases}
$$

We now express the hypothesis probability as

$$
\begin{aligned}
P_{0} & =\operatorname{Pr}\left[H_{0} \mid \theta>\theta_{t}\right] \\
& =\frac{\operatorname{Pr}\left[\theta>\theta_{t} \mid H_{0}\right]}{\operatorname{Pr}\left[\theta>\theta_{t} \mid H_{0}\right]+\operatorname{Pr}\left[\theta>\theta_{t} \mid H_{1}\right]}
\end{aligned}
$$

where we have omitted the location index $(0,0)$ and $\operatorname{Pr}\left[H_{0}\right]=\operatorname{Pr}\left[H_{1}\right]=1 / 2$ reflects that we have no apriori information about the presence of a tap. We can evaluate (4.178) by using (4.177) if we find the probability distribution for $\theta$. $U_{l, k}$ are mutually uncorrelated and WSS by the WSSUS assumption, and if the noise driving the AR(1) model in (4.86) is Gaussian they are in addition Gaussian random variables. The purpose of (4.177) is to find $P_{0}$, and this probability is a function of the scattering strength for the path corresponding to $U_{0,0}$ relative to the scattering strengths of the other paths. The scattering strength is between 0 and 1 , and it is given by the variance of $U$ in (4.88). An interesting scenario is when we are trying to detect a weaker signal return in the presence of a number of stronger returns. Therefore we 
assume that the Gaussian random variables $U_{l, o}$ for $(l, o) \neq(0,0)$ have unit variance and that $U_{0,0}$ has variance $u_{L-1}^{2}$. Thus we have

$$
\begin{aligned}
\left|U_{0,0}\right|^{2} / u_{L-1}^{2} & \sim \chi_{2}^{2} \\
\sum_{(, o) \neq(0,0)}\left|U_{l, o}\right|^{2} /(L-1) & \sim \chi_{2}^{2} .
\end{aligned}
$$

We observe from (4.177) that $\theta$ is a sum of differently scaled $\chi_{2}^{2}$ variables under both hypotheses, and we can write

$$
\begin{aligned}
& \operatorname{Pr}\left[\theta>\theta_{t} \mid H_{0}\right]=\epsilon_{0} e^{-\theta_{t} / 2 a}+\beta_{0} e^{-\theta_{t} / 2 b}+\gamma_{0} e^{-\theta_{t} / 2 c} \\
& \operatorname{Pr}\left[\theta>\theta_{t} \mid H_{1}\right]=\beta_{1} e^{-\theta_{t} / 2 b}+\gamma_{1} e^{-\theta_{t} / 2 c}
\end{aligned}
$$

where

$$
\begin{aligned}
\epsilon_{0} & =\frac{1}{(1-b / a)(1-c / a)} \\
\beta_{0} & =\frac{1}{(1-a / b)(1-c / b)} \\
\gamma_{0} & =\frac{1}{(1-a / c)(1-c / b)} \\
\beta_{1} & =\frac{1}{(1-c / b)} \\
\gamma_{1} & =\frac{1}{(1-b / c)}
\end{aligned}
$$

and

$$
\begin{aligned}
a & =\left(M \mathcal{E} u_{L-1}\right)^{2} \\
b & =(L-1) M \mathcal{E}^{2} \\
c & =M \mathcal{E} \sigma_{w}^{2} .
\end{aligned}
$$

Fig. 4-27 shows $P_{0}$ vs the peak height $\theta_{t} /(M \mathcal{E})^{2}$ parameterized by the training 


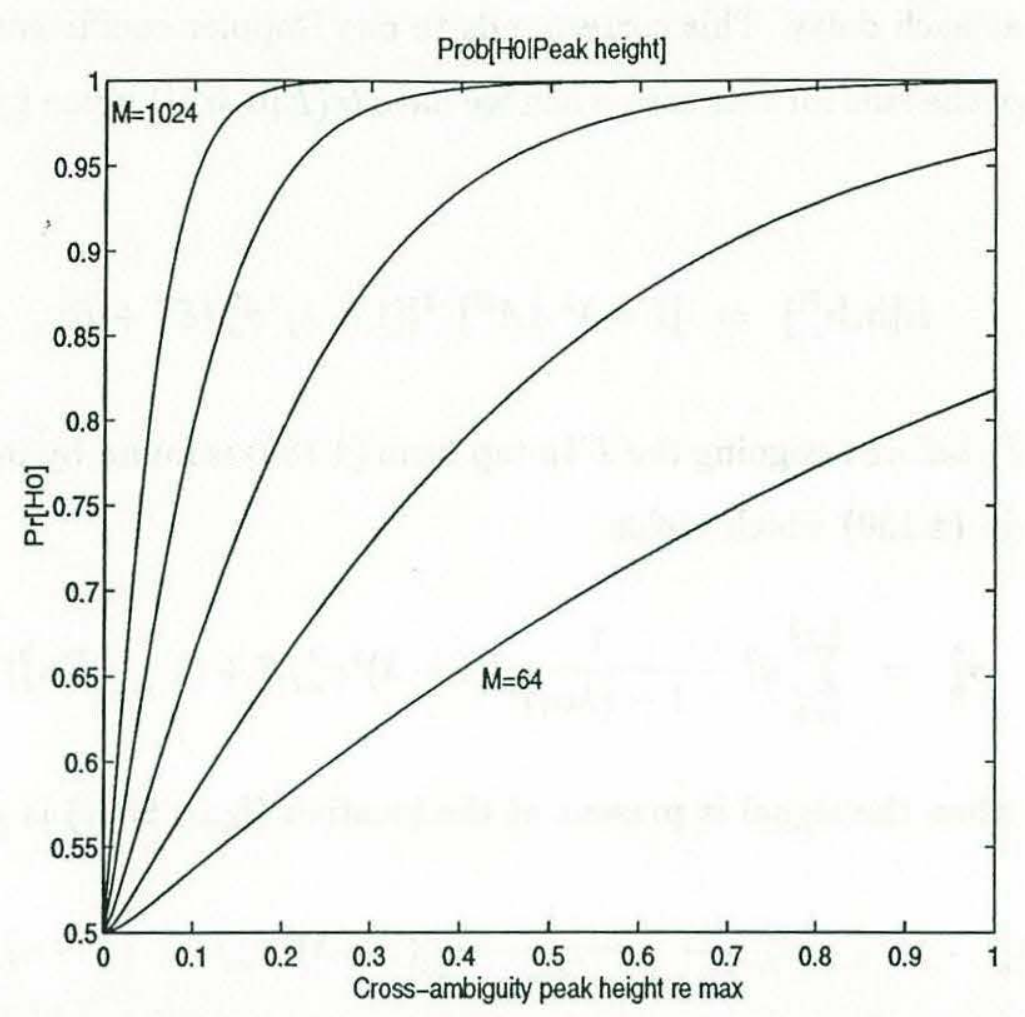

Figure 4-27: The probability that a certain location in the cross-ambiguity function corresponds to a signal return vs the value of the cross-ambiguity function. There are $L=9$ unit strength scatterers at other locations and the scatterer at $(0,0)$, which we are trying to detect has strength 0.5 . The normalization of the first axis is relative the ambiguity function peak value.

sequence length $M$ for an SNR of $6 \mathrm{~dB}, u_{L-1}^{2}=.5$ and $L=10$. We note that in the tap assignment situation $u_{i}^{2}$ is not given, and we use the sample variance estimated by the cross-ambiguity function as shown in (4.190). This completes the specification of the rule (4.161) which is now determined by the cross-ambiguity function, and we present two examples illustrating the result of using the rule.

Example 1: One Doppler coefficient for each delay The numerical approach necessary to find the solution of (4.152) precludes the presentation of a more transparent analytical form of (4.161). However, as discussed in Section 4.3.8, an analytical form of $E\left[\mathbf{h}_{e} \mathbf{h}_{e}^{H}\right]$ is found when $\tilde{P}$ is diagonal corresponding to one Doppler 
coefficients at each delay. This corresponds to one Doppler coefficient at each delay. We now show the rule for this case when we have $\operatorname{tr}\left(E\left[\mathbf{h}_{e} \mathbf{h}_{e}^{H}\right]\right)$ given by (4.231) which yields

$$
E\left[\mathbf{h}_{e} \mathbf{h}_{e}^{H}\right]=\left[I-\lambda^{2} A A^{H}\right]^{-1}\left[(1-\lambda)^{2} \sigma_{w}^{2} / \mathcal{E} I+R\right] .
$$

The energy $\mathcal{E}_{\hat{h}}$ before assigning the $L$ 'th tap from (4.150) is found by inserting (4.183) and (4.156) in (4.150) which yields

$$
\sigma_{\hat{h}}^{2}=\sum_{i=0}^{L-2} u_{i}^{2}-\frac{1}{1-\left(\lambda \alpha_{i}\right)^{2}}\left[(1-\lambda)^{2} \sigma_{w}^{2} / \mathcal{E}+\left(1-\alpha_{i}^{2}\right) u_{i}^{2}\right] .
$$

The energy when the signal is present at the location $\left(l_{L-1}, k_{L-1}\right)$ is given by

$$
\begin{aligned}
& A_{1}: \sigma_{\hat{h}}^{2}=\mathcal{E}_{\hat{h}}+u_{L-1}^{2}-\frac{1}{1-\left(\lambda \alpha_{L-1}\right)^{2}}\left[(1-\lambda)^{2} \sigma_{w}^{2} / \mathcal{E}+\left(1-\alpha_{L-1}^{2}\right) u_{L-1}^{2}\right] \\
& A_{2}: \sigma_{\hat{h}}^{2}=\mathcal{E}_{\hat{h}} .
\end{aligned}
$$

corresponding to (4.154). We get the expression under $A_{1}$ by using (4.184) and the definition of $\mathcal{E}_{\hat{h}}$ in (4.150), and observing that the only difference is that the index $i$ ends at $L-1$. The expression under $A_{2}$ reflects that nothing has changed. The energy loss is

$$
\Delta_{0}=u_{L-1}^{2}-\frac{1}{1-\left(\lambda \alpha_{L-1}\right)^{2}}\left[(1-\lambda)^{2} \sigma_{w}^{2} / \mathcal{E}+\left(1-\alpha_{L-1}^{2}\right) u_{L-1}^{2}\right]
$$

corresponding to (4.155). The energy in the two cases $A_{1}$ and $A_{2}$ when the signal is absent is now given by

$$
\begin{aligned}
& A_{1}: \sigma_{\hat{h}}^{2}=\mathcal{E}_{\hat{h}}-\frac{\alpha_{L-1}^{2}}{1-\left(\lambda \alpha_{L-1}\right)^{2}}(1-\lambda)^{2} \sigma_{w}^{2} / \mathcal{E} \\
& A_{2}: \sigma_{\hat{h}}^{2}=\mathcal{E}_{\hat{h}}
\end{aligned}
$$


corresponding to (4.157). The expression under $A_{1}$ follows from (4.184) by ending the index $i$ at $L-1$ with $u_{L-1}^{2}=0$ since the signal is absent. The energy loss corresponding to (4.158) is

$$
\Delta_{1}=\frac{\alpha_{L-1}^{2}}{1-\left(\lambda \alpha_{L-1}\right)^{2}}(1-\lambda)^{2} \sigma_{w}^{2} / \mathcal{E}
$$

We now write the rule (4.161) by using (4.186) and (4.188) as

$$
\begin{aligned}
& P_{0} u_{L-1}^{2}-\frac{1-\lambda}{(1+\lambda)\left(\mathcal{E} / \sigma_{w}^{2}\right) \alpha_{L-1}^{2}}>0: A_{1} \\
& P_{0} u_{L-1}^{2}-\frac{1-\lambda}{(1+\lambda)\left(\mathcal{E} / \sigma_{w}^{2}\right) \alpha_{L-1}^{2}}<0: A_{2}
\end{aligned}
$$

where we see from (4.180)-(4.182) that $P_{0}$ is a function of the scattering strength $u_{L-1}^{2}$. We can find $u_{L-1}^{2}$ from (4.189), and the interpretation is to include a tap if its scattering strength is above a threshold depending on the Doppler spread of the tap, the SNR and the tracking bandwidth that will be used in the receiver. For representative symbol rates and Doppler spreads the range of possible values for $\alpha$ is very small and close to 1 . It is therefore a good approximation to set $\alpha_{L-1}^{2}=1$ in (4.189).

The scattering strength is not readily available, and it can be estimated by the sample mean of the cross-ambiguity function since (4.177) under $H_{0}$ yields

$$
E[\theta(0,0)]=u_{L-1}^{2}(M \mathcal{E})^{2}+M \mathcal{E}^{2} \sum_{i=0}^{L-2} u_{i}^{2}+M \mathcal{E} \sigma_{w}^{2} \approx u_{L-1}^{2}(M \mathcal{E})^{2}
$$

The expected value of the cross-ambiguity function is a biased estimator for $u_{L-1}^{2}$ where the bias is small when $L-1 \ll M$. By substituting the sample mean for the expected value in (4.190) we can estimate $u_{L-1}^{2}$ and use the rule (4.189) to assign taps in the channel tracker. 
Example 2: All Doppler coefficients at a single delay The other extreme of $\tilde{P}$ is when this matrix is fully populated, corresponding to a purely Doppler spread channel. In this case (4.152) is solved numerically, and to further illustrate (4.161) we show in Fig. 4-28 the number of taps as given by (4.161) vs $\lambda$ for this case. We show in Section 4.3.3 that the tracking bandwidth for each tap of the TU-RLS decreases with increasing $\lambda$, therefore we see an increasing number of taps when $\lambda$ increases. The case shown in Fig. 4-28 is for an SNR of $6 \mathrm{~dB}$ and a Doppler spread of $5 \mathrm{~Hz}$ at a symbol rate of $2500 \mathrm{symb} / \mathrm{sec}$.

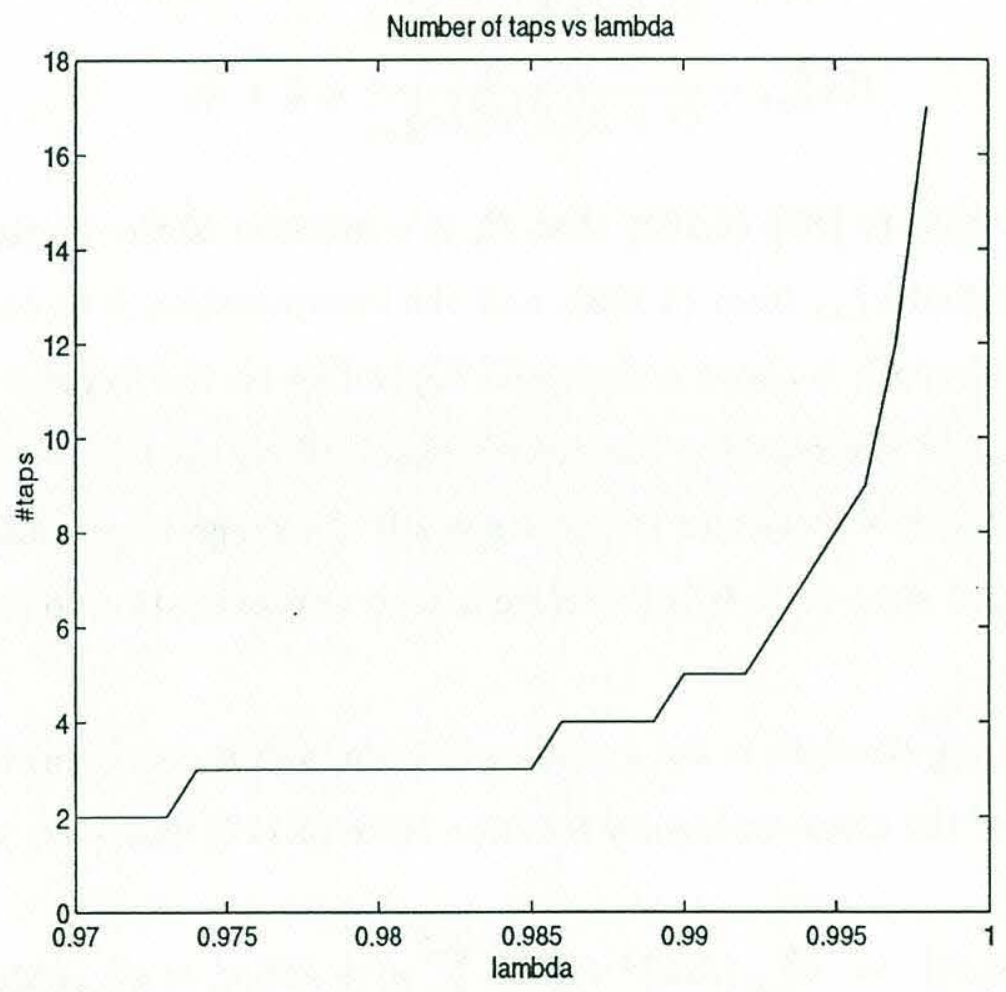

Figure 4-28: The number of taps used by the receiver vs $\lambda$ for a Doppler spread of $5 \mathrm{~Hz}$ and a SNR of $6 \mathrm{~dB}$.

Summary We assume that the cross-ambiguity function is available, and at each location of this function we perform a binary hypothesis test on signal presence of absence. The two possible errors of this test is to omit a tap at a location where 
signal is present or include a tap at a location where signal is absent. Both errors will give energy loss in the data input to the decoder. We compute the losses which are used in a cost function, and we derive a rule based in the minimization of the expected va'tue of tiis cost. The rule makes use of the hypothesis probability, and we express this in terms of the cross-ambiguity function. We show two examples of the rule (4.161). In the first example, which is the case of one Doppler coefficient at each delay, the rule (4.189) suggests that we threshold each location $\left(l_{L-1}, k_{L-1}\right)$ of the sample mean of the cross-ambiguity function. The threshold is given by the SNR and the tracking bandwidth as shown in (4.189). In the second example, which is the case of all Doppler coefficients at the same delay, we show the number of taps resulting from the rule (4.161) vs the tracking bandwidth represented by $\lambda$.

By assigning probabilities to error occurrences and costs to the energy losses a rule for tap selection is derived.

\subsubsection{Tap tracking}

Motivation The next task is then to update the initial channel response estimate by tracking the channel parameters Doppler and delay. This could be carried out on a sample by sample recursive basis by assuming statistical models for the evolution of Doppler and delay vs time. The discussion of the DFE in Section 4.2 shows that this may give unexpected system behavior. The case of tracking Doppler on a sample by sample basis leads directly to the use of a PLL since this is the result of the TU-RLS applied to this problem. The analysis pertaining to the DFE suggests not to use coupled adantive algorithms running simultaneously on the same data. In our case we already have the TU-RLS (4.92) and (4.93) running to track the delayDoppler-spread function $U_{l, k}(n)$, therefore we constrain ourselves to only updating delay and Doppler estimates on a less frequent basis than from sample to sample.

We now derive a tracking algorithm for the delays and the Doppler shifts. We use information from previous estimates, so we avoid retraining entirely for each 
estimate but use the information from the last estimate in the computation of the new one.

\section{Doppler and delay tracking}

According to the model (4.83) the Doppler shifts $\nu=\left[\nu_{0} \cdots \nu_{L-1}\right]^{T}$ are known constants. In a realistic situation depending on the relative transmitter, receiver and boundary motion it may be necessary to allow $\boldsymbol{\nu}$ to vary slowly. The initialization of the channel tracker by means of the choice of the number of taps and their locations in delay and Doppler is described in Section 4.3.6. One interpretation of this procedure is to compute the cross-ambiguity function (4.163) and then perform a thresholded search (4.161) in delay and Doppler over this surface. In this scenario, where we assume the initialization to be correct, the task is to update the location of the taps. Therefore, from the initialization we know $\boldsymbol{\nu}=\boldsymbol{\nu}_{0}$ at time $n$, and we assume

$$
\nu=\nu_{0}+\delta \nu
$$

at time $n+N-1$ where $\delta \boldsymbol{\nu} \ll \boldsymbol{\nu}_{0}$ is an unknown constant vector. The $N$ new data samples are used to compute a restricted version of the updated cross-ambiguity function. The restriction is to only compute the cross-ambiguity function over the samples $\delta \boldsymbol{\nu}$. By this constraint we use the information from previous initialization, and we save computations by this constraint. Likewise we utilize the previous estimate in delay and write the new delays as

$$
\mathrm{n}=\mathrm{n}_{0}+\delta \mathrm{n}
$$

where $\mathrm{n}_{0}$ is the estimate at time $n$ and $\mathrm{n}$ is the estimate at time $n+N-1$. Thus the updated cross-ambiguity function is computed in a number of smaller areas $(\delta \mathbf{n}, \delta \boldsymbol{\nu})$ centered at $\left(\mathrm{n}_{0}, \boldsymbol{\nu}_{\mathrm{o}}\right)$, and the taps in these areas are relocated in the same areas based on the new cross-ambiguity function in the same way as described in Section 4.3.6. 
A typical scenario for the communication system is data transmitted in packets of a certain length with a fixed pause between packets. The update described above is a constrained search over a set of tap locations starting at the old positions of the taps.

\subsubsection{Receiver robustness}

Preview Section 4.3.6-4.3.7 describe the initialization and operation of the receiver, and we now turn to the evaluation. In a practical scenario there will be estimation errors in the parameters used to initialize the receiver, and noise, delay spread and Doppler spread are always present in the data. This limits the receiver capability even with perfect initialization. The receiver is built up of a channel tracker, a linear MMSE decoder and a quantizer that are connected as shown in Fig. 4-20. One objective of this section is to find the limits of how much Doppler spread, delay spread and noise this receiver can handle before it stops working satisfactorily when it is in the tracking mode. Thus we assume correct initialization of tap locations, and we also assume that the channel tracker has converged by means of the training sequence. One important assumption when the receiver is decoding

is that the decoded sequence $\tilde{z}(n)$ is close to the transmitted sequence $z(n)$ because the channel tracker relies on this to give good channel estimates. This way of decision feedback is known to cause error propagation in the DFE that was analyzed earlier. We observe that the receiver in Fig. 4-20 is likely to be more robust than the DFE in Fig. 4-13 because a DFE uses the feedback of symbols in both channel tracking, similar to the receiver in Fig. 4-20, and also directly in the decoding of symbols. This last feedback path is not present in the receiver in Fig. 4-20, and this justifies the improved robustness. The receiver structure makes it natural to attempt to analyze each of its three parts separately. We view them as three separate systems which are the MMSE decoder, the quantizer and the channel tracker, and the assumptions associated with the characterization of each device will be stated 
when they are used. Thus the MMSE decoder is a linear filter with a given impulse response, and the received data is the input whereas the soft estimate of the transmit sequence is the output. The main result for this unit is in (4.199) which is a well known result from Wiener filtering and least squares estimation [104], [100]. The quantizer is a nonlinear system with the soft estimate of the transmit sequence as the input and the quantized transmit sequence estimate as the output. It will produce the correct transmit sequence with a certain probability, and this probability is computed [82], [100] and given in (4.203) which is the main result for this device. The channel tracker is an adaptive filter using TU-RLS. Certain results from steady state analysis [64], [65] and also some results derived in this thesis are used to quantify the error in the channel estimate, and the main result is given in (4.221). This equation suggests that the channel estimation error covariance is evolving as a first order recursive, coupled matrix equation, and it is verified in Fig. 4-30. The stability is determined by the eigenvalue spread of the matrix $\tilde{P}$ in (4.218) which is block diagonal. The two extremes of diagonal and fully populated $\tilde{P}$ are discussed. When this matrix is diagonal the combination of the results (4.199), (4.203) and (4.221) yields an equation for the error probability involving the SNR, the Doppler spread and the delay spread in (4.232) which is the main result for this case. When $\tilde{P}$ is fully populated the receiver stability is determined by the condition number of this matrix, and this is demonstrated in Fig. 4-31. The main result (4.221) is illustrated by a purely Doppler spread noisy channel in Fig. 4-34 and a more general doubly spread channel in Fig. 4-35. This equation gives an exhaustive characterization of the receiver performance when the receiver is in the tracking mode, and it is valid for the general doubly spread noisy channel under the assumptions stated throughout the section. Before we turn to this issue of channel distortion we present an example of initialization error in Fig. 4-29. This example illustrates that the receiver can tolerate a certain amount of estimation error in the initialization, i.e., perfect channel knowledge is not necessary. 


\section{Doppler mismatch}

There will be mismatch due to noise in the estimation of $\boldsymbol{\nu}$ and $\mathrm{n}$, and we now consider a situation where the Doppler shifts have been estimated and there is an error

$$
\nu_{e}=\hat{\nu}-\nu
$$

where $\boldsymbol{\nu}$ are the true Doppler shifts and $\hat{\boldsymbol{\nu}}$ are the estimates. We know from the analysis of the RLS in presence of Doppler spread, and also from the steady state expression (4.96) that the residual Doppler $\nu_{e}$ enters the TU-RLS to give tap rotation: If the transmitted signal $z(n)$ is a whitened sequence and there is one Doppler

coefficient per delay the expected value of the steady state vector equation for $\hat{\mathbf{h}}(n)$, given by (4.96), decouples to a set of scalar equations therefore we consider the scalar case which yields

$$
E[\hat{h}(n+1)]=\lambda e^{j 2 \pi \hat{\nu}} E[\hat{h}(n)]+(1-\lambda) e^{j 2 \pi \nu n} .
$$

As the analysis in (4.44)-(4.51) shows the steady state tap amplitude is affected. Fig. 4-9 shows the maximum relative Doppler shift at a given forgetting factor $\lambda$ before a DFE would break down because of error propagation. The maximum Doppler mismatch that can be handled by the TU-RLS in (4.92) is a function of how much attenuation of the steady state value of $\hat{\mathrm{h}}(n)$ that can be tolerated. If we arbitrarily require that $|E[\hat{h}(n)]|$ stays above $90 \%$ of its true value the contours of maximum allowable $\lambda$ vs symbol rate and percent Doppler mismatch $100 \nu_{e} / \nu$ is shown in Fig. 429. When the modulation is QPSK one symbol corresponds to 2 bit, so the vertical axis represents bit rate. As discussed in relation to Fig. 4-9 it is the Doppler relative to the sample rate of the received signal that is significant, so a potential way to deal with Doppler at the expense of computational load and number of degrees of 


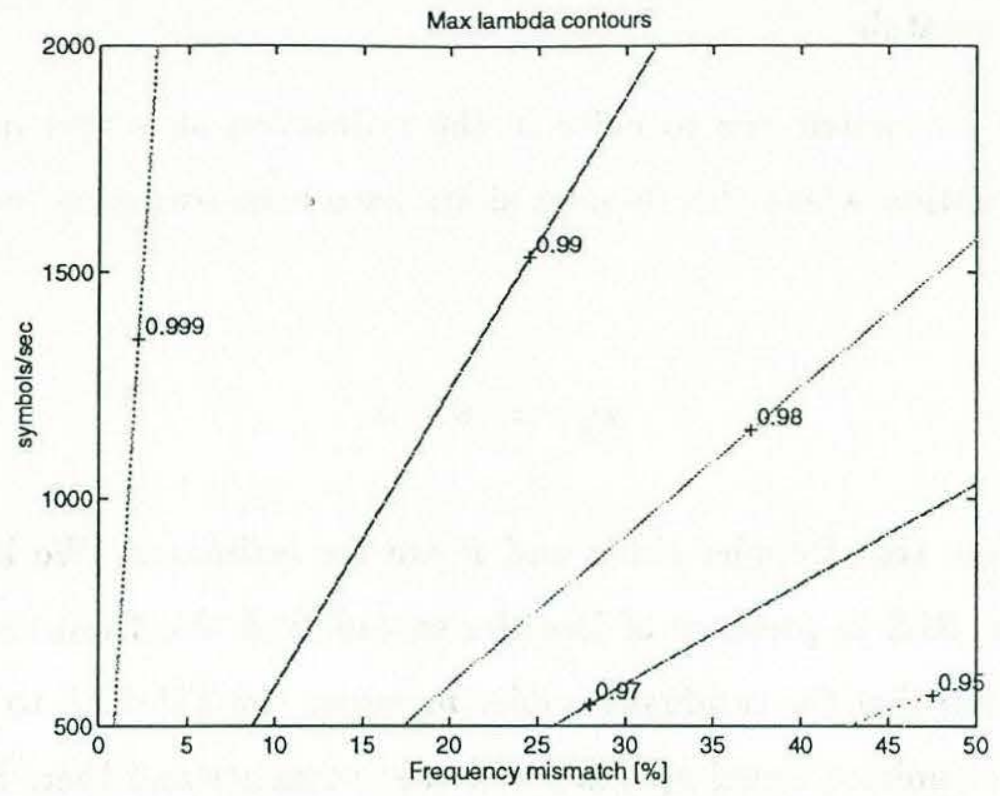

Figure 4-29: Contours of maximum $\lambda$ for a given symbol rate and Doppler frequency mismatch that keep the amplitude of $\hat{h}(n)$ above $90 \%$ of $h(n)$.

freedom is to use higher sample rate in the receiver. The contours shown in Fig. 4-29 are for a rate of 1 sample/symbol.

We note that increasing the sample frequency of the receiver is different than increasing the symbol rate. Both these changes make a fixed Doppler spread less severe, because the change from sample to sample as seen by the TU-RLS decreases. Both the approaches have disadvantages that make them less tractable. The first approach increases the number of degrees of freedom and computational load in the receiver as discussed above. If we assume that fixed amount of energy is available at the transmitter the second approach results in less energy per symbol transmitted. This in turn increases the probability of detection error in the receiver. Therefore this approach is only attractive if more power is available as the symbol rate is increased.

We now turn to the second issue of receiver robustness which is to quantify how much noise and spread the receiver can operate under with a given probability of decoding error. 


\section{MMSE decoder}

The received signal is modeled by (4.83). The decoder is an FIR filter with given impulse response, see Section 4.3.5, and the MMSE impulse response is given by the correlation matrix $R_{y y}(n)$ and the cross correlation vector $\mathbf{r}_{z y}(n)$

$$
\begin{aligned}
\hat{\mathbf{h}}_{0}(n) & =R_{y y}(n)^{-1} \mathbf{r}_{z y}(n) \\
R_{y y}(n) & =E\left[\mathbf{y}(n) \mathbf{y}(n)^{H}\right] \\
\mathbf{r}_{z y}(n) & =E\left[z^{*}(n) \mathbf{y}(n)\right] \\
\mathbf{y}(n) & =\left[y\left(n-l_{0}\right) \cdots y\left(n-l_{L-1}\right)\right]^{T} .
\end{aligned}
$$

We introduce this filter with $L$ taps whereas it has $L_{1}$ taps in Section 4.3.5, and we introduce $L_{1}$ and comment on this difference below. The impulse response for this filter is supplied by the TU-RLS channel tracker and it contains errors so we have from (4.148) that

$$
\hat{\mathbf{h}}(n)=\mathbf{h}(n)-\mathbf{h}_{e}(n)
$$

where the error $\mathbf{h}_{e}$ is modeled as a random vector. Thus we have from (4.138) that

$$
\begin{aligned}
\hat{z}(n) & =\hat{\mathbf{h}}_{0}^{T}(n) \mathbf{y}(n) \\
& =\sqrt{\mathcal{E}} z(n)+\hat{z}_{e}(n) .
\end{aligned}
$$

The Wiener filter has the well known minimum mean square error $J_{\min }$ [45], [104], and any suboptimal filter has an error variance that is expressed in terms of $J_{\min }$ and an additional term due to the deviation from the optimal Wiener filter [45]. In our case this deviation is $\mathbf{h}_{e}(n)$, and we have that the estimation error variance is

$$
E\left[\left|\hat{z}_{e}(n)\right|^{2} \mid \mathbf{h}_{e}\right]=J \mid \mathbf{h}_{e}=\mathcal{E}-\mathbf{r}_{z y}^{H} R_{y y}^{-1} \mathbf{r}_{z y}+\mathbf{h}_{e}^{H} R_{y y} \mathbf{h}_{e}
$$




$$
=J_{\min }+\mathrm{h}_{e}^{H} R_{y y} \mathrm{~h}_{e} .
$$

$J_{\min }$ is the error variance of the MMSE filter and this is the lower bound on the error variance. The channel estimation error $\mathrm{h}_{e}$ increases the decoding error variance by $\mathbf{h}_{e}^{H} R_{y y} \mathbf{h}_{e}$. The variance of $\hat{z}_{e}(n)$ is found by taking expectation over $\mathbf{h}_{e}$ and it is given by

$$
\sigma_{e}^{2}=E\left[J \mid \mathbf{h}_{e}\right]=J_{\min }+\operatorname{tr}\left(R_{y y} E\left[\mathbf{h}_{e} \mathbf{h}_{e}^{H}\right]\right) .
$$

When the filter error $\mathbf{h}_{e}$ is zero the estimation error $\hat{z}_{e}(n)$ is a white sequence uncorrelated with $\hat{z}(n)$ and it is modeled as a Gaussian random process. This is approximately true when $\mathbf{h}_{e}$ is small, and this characterization of $\hat{z}_{e}(n)$ is used in the following.

We know that $J_{\min }$ is the MMSE obtained when using the Wiener filter but in our case, where we are using a finite length FIR filter, this result leaves the filter order undetermined [82]. It only states that with a given number of taps $L_{1}$ in the linear MMSE decoder in Fig. 4-20 $J_{\min }$ is the smallest obtainable error variance. $J_{\min }$ decreases with $L_{1}$ and the decoder is sometimes used with $L_{1}>L$ taps. There is no need for the channel tracker to have more than $L$ taps, therefore we modify (4.198) and (4.199) to yield

$$
\begin{aligned}
E\left[\left|\hat{z}_{e}(n)\right|^{2} \mid \mathbf{h}_{e}\right] & =J \mid \mathbf{h}_{e}=\mathcal{E}-\mathbf{r}_{z y}^{H} R_{y y}^{-1} \mathbf{r}_{z y}+\mathbf{h}_{e}^{H} \tilde{R}_{y y} \mathbf{h}_{e} \\
& =J_{\min }+\mathbf{h}_{e}^{H} \tilde{R}_{y y} \mathbf{h}_{e} \\
\sigma_{e}^{2} & =E\left[J \mid \mathbf{h}_{e}\right]=J_{\min }+\operatorname{tr}\left(\tilde{R}_{y y} E\left[\mathbf{h}_{e} \mathbf{h}_{e}^{H}\right]\right) .
\end{aligned}
$$

$R_{y y}$ has Toeplitz structure, and we construct $\tilde{R}_{y y}$ from $R_{y y}$ by noting that $\tilde{R}_{y y}$ has the same diagonals as $R_{y y}$ for all diagonals $n \leq L$. For example, if $L=2$ and $L_{1}=3$ 
we have

$$
\begin{aligned}
& \tilde{R}_{y y}=\left[\begin{array}{ll}
R_{11} & R_{12} \\
R_{21} & R_{11}
\end{array}\right] \\
& R_{y y}=\left[\begin{array}{ccc}
R_{11} & R_{12} & 0 \\
R_{21} & R_{11} & R_{12} \\
0 & R_{21} & R_{11}
\end{array}\right]
\end{aligned}
$$

\section{Quantizer}

The operation of the quantizer is to assign $\hat{z}(n)$ to one of the symbols in the alphabet, and for QPSK we have

$$
z(n) \in\{-j,-1, j, 1\}
$$

The quantizer uses as criterion for assignment the minimum distance rule [40], which is that the value of $z(n)$ that is closest to $\hat{z}(n)$ is chosen. This is the well known minimum distance receiver, and when the error is assumed to be Gaussian the error

probability is [82], assuming that each symbol drawn from (4.202) is transmitted with energy $\mathcal{E}$,

$$
P_{e}=\operatorname{Pr}[\tilde{z}(n) \neq z(n)]=\operatorname{erfc}\left(\sqrt{\frac{\mathcal{E}}{2 \sigma_{e}^{2}}}\right)\left(1-\frac{1}{2} \operatorname{erfc}\left(\sqrt{\frac{\mathcal{E}}{2 \sigma_{e}^{2}}}\right)\right)
$$

which is a function of the $\operatorname{SNR} \mathcal{E} / \sigma_{e}^{2}$ only. The complementary error function erfc is given by

$$
\operatorname{erfc}(x)=\frac{2}{\sqrt{\pi}} \int_{x}^{\infty} e^{-t^{2}} d t
$$


Thus we can interpret the quantizer output as

$$
\tilde{z}(n)=z(n)+z_{e}(n)
$$

where $z_{e}(n)$ is the error sequence from the quantizer output. It is assumed to be independent of $z(n)$ and it is modeled as a stationary, white random process with

$$
\begin{aligned}
z_{e}(n) & \in\{-2,-2 j,-1-j, 1-j, 0, j-1, j+1,2 j, 2\} \\
\operatorname{Pr}\left[z_{e}(n)=0\right] & =1-P_{e} .
\end{aligned}
$$

In the literature on quantizers the quantity $z_{e}(n)$ is called the granular noise, and the assumption amounts to consider the granular noise to be independent of the quantizer level $z(n)$ [50]. The probability mass function of $z_{e}(n)$ follows from simple counting arguments assuming that all the sample values of $z(n)$ are equally likely. It is given by

$$
\begin{aligned}
P_{z_{e}} & =P_{e}\left[\frac{1}{12}, \frac{1}{12}, \frac{1}{6}, \frac{1}{6}, \frac{1}{P_{e}}-1, \frac{1}{6}, \frac{1}{6}, \frac{1}{12}, \frac{1}{12}\right] \\
E\left[z_{e}(n)\right] & =0 \\
E\left[\left|z_{e}(n)\right|^{2}\right] & =\frac{8}{3} P_{e}=\gamma P_{e}
\end{aligned}
$$

where each vector component of $P_{z_{e}}$ is the probability of $z_{e}(n)$ taking on the value of the corresponding entry in (4.206) and $\gamma=8 / 3$ is a constant depending on the modulation format that is used. The actual value of $\gamma$ is given in a straightforward manner from the evaluation of the variance in (4.207). To illustrate this we consider another example of binary PSK modulation. In this case (4.206) yields

$$
z_{e, P S K}(n) \in\{-2,0,2\}
$$


and (4.207) yields

$$
P_{z_{e, P S K}}=P_{e}\left[\frac{1}{2}, \frac{1}{P_{e}}-1, \frac{1}{2}\right]
$$

From this we get $E\left[\left|z_{e, P S K}(n)\right|^{2}\right]=\left((-2)^{2} \frac{1}{2}+(2)^{2} \frac{1}{2}\right) P_{e}=4 P_{e}$ so that $\gamma=4$ for binary PSK. The error probability $P_{e}$ depends on the channel estimation error, and we now find an expression for this.

\section{Channel tracker}

The channel model is given by (4.84) and (4.87) but we explicitly want to show the noișe originating from decision errors, therefore we denote the true symbol $\mathbf{c}^{H}(n)$ with

$$
\mathbf{c}_{0}^{H}(n)=\sqrt{\mathcal{E}}\left[z\left(n-l_{0}\right) \cdots z\left(n-l_{L-1}\right)\right] .
$$

The equation (4.87) models the channel variation as a collection of $\mathrm{AR}(1)$ processes with parameters $\alpha_{i}$. The TU-RLS channel tracker operates according to (4.92) and (4.93) where

$$
\begin{aligned}
\mathbf{c}^{H}(n) & =\sqrt{\mathcal{E}}\left[\tilde{z}\left(n-l_{0}\right) \cdots \tilde{z}\left(n-l_{L-1}\right)\right] \\
& =\mathbf{c}_{0}^{H}(n)+\mathbf{c}_{e}^{H}(n) \\
\mathbf{c}_{e}^{H}(n) & =\sqrt{\mathcal{E}}\left[z_{e}\left(n-l_{0}\right) \cdots z_{e}\left(n-l_{L-1}\right)\right] .
\end{aligned}
$$

We now split the error $e(n)$ and the gain $\mathrm{k}(n)$ in two terms in order to identify the error from wrong decisions in the quantizer. Thus $\mathrm{k}_{0}(n)$ and $e_{0}(n)$ refers to gain and error due to imperfect channel tracking and measurement noise. These errors are well known [45], [64]. The additional error in gain $\mathrm{k}_{e}(n)$ and $e_{e}(n)$ due to decision feedback errors are written separately because we want to quantize explicitly the 
effect of decision feedback errors. We find from (4.92) and (4.93) that

$$
\begin{aligned}
e(n) & =e_{0}(n)-e_{e}(n) \\
e_{0}(n) & =y(n)-\mathbf{c}_{0}^{H}(n) \hat{\mathbf{h}}(n) \\
e_{e}(n) & =\mathbf{c}_{e}^{H}(n) \hat{\mathbf{h}}(n) \\
\mathrm{k}(n) & =\mathrm{k}_{0}(n)+\mathbf{k}_{e}(n) \\
\mathrm{k}_{\mathbf{0}}(n) & =\frac{\frac{1}{\lambda}\left(A P(n-1) A^{H}+R\right) \mathbf{c}_{\mathbf{0}}(n)}{\sigma_{w}^{2}+\frac{1}{\lambda} \mathbf{c}_{0}^{H}(n)\left(A P(n-1) A^{H}+R\right) \mathbf{c}_{\mathbf{0}}(n)} \\
\mathrm{k}_{\mathrm{e}}(n) & =\frac{\frac{1}{\lambda}\left(A P(n-1) A^{H}+R\right) \mathbf{c}_{\mathbf{e}}(n)}{\sigma_{w}^{2}+\frac{1}{\lambda} \mathbf{c}_{0}{ }^{H}(n)\left(A P(n-1) A^{H}+R\right) \mathbf{c}_{\mathbf{0}}(n)}
\end{aligned}
$$

where we have approximated

$$
\mathbf{c}^{H}(n)\left(A P(n-1) A^{H}+R\right) \mathbf{c}(n) \approx \mathbf{c}_{\mathbf{0}}{ }^{H}(n)\left(A P(n-1) A^{H}+R\right) \mathbf{c}_{\mathbf{0}}(n)
$$

The approximation in (4.213) is good when

$$
\left|\mathbf{c}_{e}(n)\right| \ll\left|\mathbf{c}_{0}(n)\right|
$$

for large $L$, and this is satisfied for representative error probabilities $P_{e} \sim 10^{-1}-10^{-4}$. In many cases $L$ is not large, and in these cases (4.213) is not satisfied. With the error rate in the range above the violation of (4.213) is infrequent enough so that the evolution of $\mathrm{k}_{0}(n)$ and $\mathrm{k}_{e}(n)$ as given in (4.212) is not severely impacted. By inserting (4.212) in (4.92) we get

$$
\begin{aligned}
\hat{\mathrm{h}}(n+1) & =A \hat{\mathrm{h}}(n)+\mathrm{k}_{0}(n) e_{0}(n)+\mathrm{k}_{0}(n) e_{e}(n) \\
& +\mathrm{k}_{e}(n) e_{0}(n)+\mathrm{k}_{e}(n) e_{e}(n) .
\end{aligned}
$$


The estimation error as given by the combination of (4.215) and (4.87) is

$$
\begin{aligned}
\mathbf{h}_{e}(n+1) & =\mathbf{h}(n+1)-\hat{\mathbf{h}}(n+1) \\
& =\left[I-\mathbf{k}_{0}(n) \mathbf{c}_{0}^{H}(n)\right]\left[A \mathbf{h}_{e}(n)+\mathbf{v}(n)\right]-\mathbf{k}_{0}(n) w(n) \\
& +\mathbf{k}_{0}(n) \mathbf{c}_{e}^{H}(n) A\left[\mathbf{h}(n)-\mathbf{h}_{e}(n)\right]-\mathbf{k}_{e}(n) \mathbf{c}_{0}^{H}(n) A \mathbf{h}_{e}(n) \\
& -\mathbf{k}_{e}(n) \mathbf{c}_{0}^{H}(n) \mathbf{v}(n)-\mathbf{k}_{e}(n) w(n) .
\end{aligned}
$$

The first two terms in (4.216) are the standard terms as can be found in the literature [105] and the remaining terms are due to decision feedback errors. We are interested in steady state properties as $\mathrm{k}(n)$ gets small, and we show in Appendix B that the TU-RLS gain is approximately

$$
\begin{aligned}
& \mathbf{k}_{0}(n)=\chi \tilde{P}(n) \mathbf{c}_{0}(n) \\
& \mathbf{k}_{e}(n)=\chi \tilde{P}(n) \mathbf{c}_{e}(n)
\end{aligned}
$$

where $\chi$ and $\tilde{P}(n)$ are found in Appendix B and given by

$$
\begin{aligned}
\chi & =1-\lambda \\
\tilde{P}(n) & =\left[\begin{array}{lll}
\tilde{P}_{0}(n) & & \\
& \ddots & \\
& & \tilde{P}_{L-1}(n)
\end{array}\right] .
\end{aligned}
$$

We have that $\operatorname{dim}\left(\tilde{P}_{l}(n)\right)=K_{l} \times K_{l}$ and $K_{l}$ is the number of Doppler coefficients at delay $l$. $\tilde{P}(n)$ is a block diagonal matrix whose structure is given by the distribution $(l, k)$ of the taps. We note that our earlier derivation (4.35) and (4.29) suggests the same values for $\chi$ and $\tilde{P}(n)$ in the case of this matrix being diagonal, but that this result is valid only for $|A|=I$. The assumption that the gain vectors are well modeled by (4.217) is tested by the simulation of the estimation error in Fig. 4-30 and we comment further on this in the discussion pertaining to this figure. By the 
assumption that the error $z_{e}(n)$ and data $z(n)$ are uncorrelated

$$
E\left[\mathbf{c}_{0}(n) \mathbf{c}_{e}^{H}(n)\right]=0
$$

and in addition we assume

$$
\begin{aligned}
E\left[\mathbf{v}(n) w^{*}(n)\right] & =0 \\
E\left[\hat{\mathbf{h}}(n) \mathbf{h}_{e}^{H}(n)\right] & =0 .
\end{aligned}
$$

The assumptions in (4.220) are often adopted in similar analyzes, and they amount to the measurement and the process noise being uncorrelated and the channel estimate and the channel estimation error being uncorrelated. The channel estimation error covariance is $\Pi(n)=E\left[\mathbf{h}_{e}(n) \mathbf{h}_{e}^{H}(n)\right]$ and it can be found from (4.216). By squaring and taking expectation of (4.216) we get

$$
\begin{aligned}
\hat{\Pi}(n) & =\left[I-\chi \tilde{P}(n) Q_{0}\right] A \hat{\Pi}(n-1) A^{H}\left[I-\chi \tilde{P}(n) Q_{0}\right]^{H} \\
& +\chi^{2} \tilde{P}(n) Q_{0} \tilde{P}^{H}(n) \sigma_{w}^{2}+R \\
& +\chi^{2} \tilde{P}(n) \dot{\mathcal{E}}^{2} \gamma P_{e}\left(L+2 \operatorname{tr}\left(A \hat{\Pi}(n-1) A^{H}\right)\right) \tilde{P}^{H}(n) \\
& +\chi^{2} \tilde{P}(n) Q_{e} \sigma_{w}^{2} \tilde{P}^{H}(n)
\end{aligned}
$$

where we have defined

$$
Q_{0}=E\left[\mathbf{c}_{0}(n) \mathbf{c}_{0}^{H}(n)\right] .
$$

$\hat{\Pi}$ is an approximation to $\Pi$ where we have neglected certain dependencies between variables, see Appendix B for the detailed derivation of (4.221). It has been proven [65] that $\hat{\Pi}(n)$ is close to $\Pi(n)$ for $A=I$ and $P_{e}=0$, and this further motivates the choice in (4.217).

We show in Appendix B that $\tilde{P}$ can be interpreted as the inverse of a sliding 
window average that is an estimate of the input signal covariance matrix, and this is also pointed out in the literature [64], [104]. Essentially the recursive schemes such as TU-RLS perform an approximate solution to the Wiener-Hopf equation because this equation yields the MMSE estimate. To solve this equation the inversion of a correlation matrix is necessary, and this inversion is updated recursively by means of the matrix inversion lemma [43] in the TU-RLS algorithm. The main point in this context is that an inversion is required, and this operation requires improved numerical accuracy as the matrix $\tilde{P}^{-1}(n)$ that is to be inverted approaches a singular matrix. The structure of $\tilde{P}(n)$ is shown in (4.218), and it varies from diagonal to fully populated. The diagonal structure occurs when we have only one Doppler shift at each delay, so that $K_{l}=1 \forall l$. The fully populated structure occurs for a purely Doppler spread channel where $K_{0}=L$ and there is only one delay. These two cases are the extremes of $\tilde{P}(n)$. A convenient metric for how sensitive the inversion of a matrix is to noise and roundoff errors is the ratio of the largest to the smallest eigenvalue also known as the condition number. We observe from (4.218) that $\tilde{P}(n)$ is always block diagonal so that the condition number in the case of a doubly spread channel is given by the eigenvalues for each block. This motivates the closer study of the two cases of diagonal and fully populated $\tilde{P}(n)$ which we now present.

\section{One Doppler coefficient at each delay}

This is the case of $K_{l}=1 \forall l$, and we now observe from (4.221) that $\hat{\Pi}$ is a diagonal matrix if $R, Q_{e}, Q_{0}$ and $\tilde{P}$ are diagonal. $R$ is always diagonal by the WSSUS assumption, from (4.222) $Q_{0}$ is diagonal and by using (4.207) we get

$$
Q_{e}=E\left[\mathbf{c}_{e}(n) \mathbf{c}_{e}^{H}(n)\right]=\mathcal{E}_{\gamma} P_{e} I
$$

We also note that $\hat{\Pi}(n)$ is given by a set of first order linear, coupled, recursive difference equations, therefore the stability of (4.221) is given by the eigenvalues of 
the system matrix $A_{0}$ which we now proceed with defining. By using (B.8) for $\tilde{P}$, and observing that (4.222) yields $Q_{0}=\mathcal{E} I,(4.221)$ yields

$$
\begin{aligned}
\hat{\Pi}(n) & =\lambda^{2} A \hat{\Pi}(n-1) A^{H}+2(1-\lambda)^{2} \gamma P_{e} \operatorname{tr}\left(A \hat{\Pi}(n-1) A^{H}\right) I \\
& +(1-\lambda)^{2} \sigma_{w}^{2} / \mathcal{E} I+R+(1-\lambda)^{2} \gamma P_{e} L I \\
& +(1-\lambda)^{2} \gamma P_{e} \sigma_{w}^{2} / \mathcal{E} I
\end{aligned}
$$

To bring out $A_{0}$ explicitly we introduce the $L \times 1$ vector $\pi(n)$ consisting of the diagonal elements of $\hat{\Pi}(n)$, so that

$$
\boldsymbol{\pi}(n)=\operatorname{diag}(\hat{\Pi}(n))
$$

where $\operatorname{diag}(x)$ means the column vector of the main diagonal of $x$ if $x$ is a matrix, and a matrix with $x$ as its main diagonal and zeros elsewhere if $x$ is a vector. By defining

$$
\alpha_{2}=\left[\alpha_{0}^{2} \cdots \alpha_{L-1}^{2}\right]^{H}
$$

we can write

$$
\operatorname{tr}\left(A \hat{\Pi}(n) A^{H}\right)=\operatorname{tr}\left(A A^{H} \hat{\Pi}(n)\right)=\boldsymbol{\alpha}_{2}^{H} \boldsymbol{\pi}(n) .
$$

Thus (4.224) is rewritten in vector version as

$$
\begin{aligned}
\boldsymbol{\pi}(n) & =\left(\lambda^{2} A A^{H}+2(1-\lambda)^{2} \gamma P_{e}\left[\begin{array}{c}
\boldsymbol{\alpha}_{2}^{H} \\
\vdots \\
\boldsymbol{\alpha}_{2}^{H}
\end{array}\right]\right) \boldsymbol{\pi}(n-1) \\
& +(1-\lambda)^{2}\left[\sigma_{w}^{2} / \mathcal{E}+\gamma P_{e}\left(\sigma_{w}^{2} / \mathcal{E}+L\right)\right] \mathbf{1}+\operatorname{diag}(R)
\end{aligned}
$$


where 1 is a column vector of $L$ 1's. We get

$$
A_{0}=\lambda^{2} A A^{H}+2(1-\lambda)^{2} \gamma P_{e}\left[\begin{array}{c}
\boldsymbol{\alpha}_{2}^{H} \\
\vdots \\
\boldsymbol{\alpha}_{2}^{H}
\end{array}\right] \text {. }
$$

Thus the stability of the difference equation for the channel estimation error covariance is given by the constraint

$$
\left|\kappa_{i}\left(A_{0}\right)\right|<1 \forall i
$$

where $\kappa_{i}\left(A_{0}\right)$ is the i'th eigenvalue of $A_{0}$. These eigenvalues determine the stability of the receiver in Fig. 4-20, and we can see that they depend explicitly on Doppler spread through $A$, the number of degrees of freedom through the size of $A$, tracking bandwidth through $\lambda$ and decision errors through $P_{e}$. The eigenvalues implicitly depend on the SNR and the delay spread through $P_{e}$. If (4.230) is satisfied steady state is given by

$$
\begin{aligned}
& E\left[\mathbf{h}_{e}(n) \mathbf{h}_{e}^{H}(n)\right] \approx \hat{\Pi} \\
= & {\left[I-A_{0}\right]^{-1}\left[(1-\lambda)^{2}\left(\sigma_{w}^{2} / \mathcal{E}+\gamma P_{e}\left(\sigma_{w}^{2} / \mathcal{E}+L\right)\right)\right.} \\
+ & R] .
\end{aligned}
$$

By combining (4.203), (4.199) and (4.231) we get

$$
\begin{aligned}
P_{e} & =\operatorname{erfc}\left(\sqrt{\frac{\mathcal{E}}{2 \sigma_{e}^{2}\left(P_{e}\right)}}\right)\left(1-\frac{1}{2} \operatorname{erfc}\left(\sqrt{\frac{\mathcal{E}}{2 \sigma_{e}^{2}\left(P_{e}\right)}}\right)\right) \\
\sigma_{e}^{2}\left(P_{e}\right) & =\mathcal{E}-\mathbf{r}_{z y}^{H} R_{y y}^{-1} \mathbf{r}_{z y} \\
& +\operatorname{tr}\left(\tilde { R } _ { y y } [ I - A _ { 0 } ] ^ { - 1 } \left[(1-\lambda)^{2} \sigma_{w}^{2} / \mathcal{E}+R\right.\right. \\
& \left.\left.+(1-\lambda)^{2} \gamma P_{e}\left(\sigma_{w}^{2} / \mathcal{E}+L\right)\right]\right)
\end{aligned}
$$


and this equation determines the performance of the composite receiver in Fig. 4-20 when it is stable. This equation in combination with (4.88) and (4.110) yields the error probability as a function of SNR $\mathcal{E} / \sigma_{w}^{2}$, Doppler spread $B$ and delay spread through $R_{y y}$.

We verify that the steady state given by (4.231) is representative for the receiver in Fig. 4-20 by running this receiver on data with a time-variant SNR as shown in the upper panel of Fig. 4-30. This SNR evolution models a long channel fade in a simplified manner. The channel has only one time-variant tap so that the delay spread is zero. Thus the error degradation in the SNR around $0.4 \mathrm{sec}$ is promptly reflected in the error probability shown in the middle panel of Fig. 4-30. The channel estimation error covariance given by (4.231) is shown as the dotted line in the lower panel, and the solid line in this panel is the channel error covariance estimated from the receiver output by running the receiver many times. We observe that the two curves in the lower panel are in good agreement, and this is a verification of the assumptions preceding the expression in (4.231) in this section.

Interpretation of steady state covariance Some insight of the terms on (4.231) can be obtained by deriving the connection to some well known results from the literature for the special case $|A|=I$. We now proceed with performing the simplifications necessary to bring out the connections. We approximate

$$
\begin{aligned}
\lambda^{2} & =(1-(1-\lambda))^{2}=1-2(1-\lambda)+(1-\lambda)^{2} \\
& \approx 1-2(1-\lambda)
\end{aligned}
$$

so that for $|A|=I(4.224)$ can be written

$$
\begin{aligned}
\hat{\Pi}(n) & =\hat{\Pi}(n-1)-2(1-\lambda) \hat{\Pi}(n-1)+2(1-\lambda)^{2} \gamma P_{e} \operatorname{tr}\left(A \hat{\Pi}(n-1) A^{H}\right) I \\
& +(1-\lambda)^{2} \sigma_{w}^{2} / \mathcal{E} I+R+(1-\lambda) \gamma P_{e} L I
\end{aligned}
$$



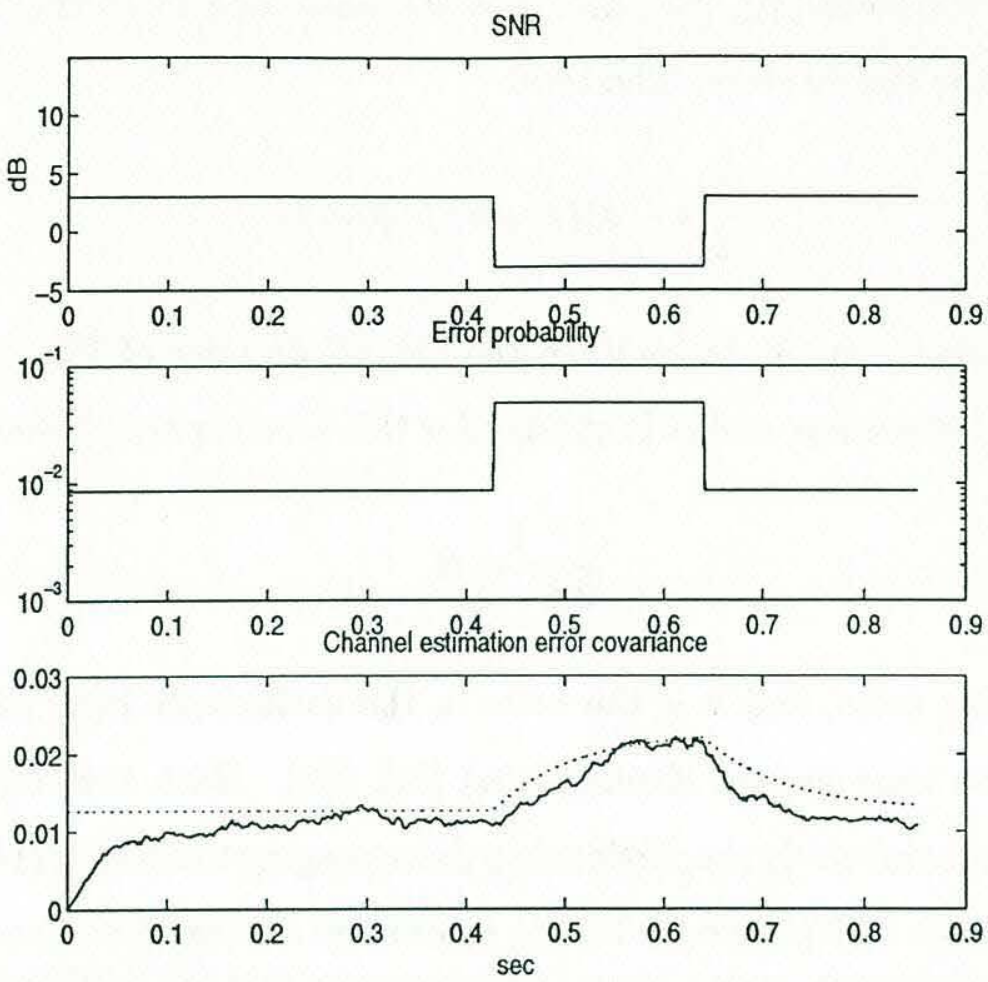

Figure 4-30: Simulation of the channel estimation error covariance for a purely Doppler spread channel. The lower panel shows the comparison between the closed form and simulated channel estimation error covariance.

$$
+(1-\lambda)^{2} \gamma P_{e} \sigma_{w}^{2} / \mathcal{E} I
$$

We neglect the term containing $\operatorname{tr}(\hat{\Pi}(n-1))$ noting that it is more than one order of magnitude smaller than the other terms involving $\hat{\Pi}(n-1)$. In steady state (4.234) yields

$$
\hat{\Pi}=\frac{1}{2}(1-\lambda) \sigma_{w}^{2} / \mathcal{E} I+\frac{1}{2} \frac{1}{1-\lambda} R+\frac{1}{2}(1-\lambda)\left(L+\sigma_{w}^{2} / \mathcal{E}\right) \gamma P_{e} I .
$$

The term

$$
\frac{1}{2}(1-\lambda) \sigma_{w}^{2} / \mathcal{E} I
$$


is called the self noise [31], [63], and it is the noise due to coefficient variation in $\hat{\mathbf{h}}(n)$ around the steady state. The term

$$
\frac{1}{2}(1-\lambda)\left(L+\sigma_{w}^{2} / \mathcal{E}\right) \gamma P_{e} I
$$

is the noise due to wrong decisions in the MMSE decoder of Fig. 4-20. It will be called the decision noise, and it is specific for this receiver structure. The term

$$
\frac{1}{2} \frac{1}{1-\lambda} R
$$

is called the lag noise, and it is the noise in the coefficients $\hat{\mathbf{h}}(n)$ due to imperfect tracking of the time-variant channel $\mathbf{h}(n)$ [31], [63]. Both the self noise and the decision noise are inversely proportional to the averaging window $1 /(1-\lambda)$, and this is in correspondence with physical intuition since more averaging will cancel noise when the noise process is white. The lag error is proportional to the averaging window, and this is also physically intuitive; since the noise is due to lack of tracking it decreases if the tracking is made faster by shortening the averaging window. Thus we observe in (4.235) the well known tradeoff between tracking bandwidth and noise robustness inherent in any adaptive algorithm, as well as the effect of decision feedback errors.

We now return to the case of general $\hat{\Pi}$ as given by (4.221), and to gain further insight into the form of this equation we turn to the other extreme of $\tilde{P}(n)$ occurring for a purely Doppler spread channel.

\section{All Doppler coefficients at a single delay}

In this case $\tilde{P}(n)$ is fully populated, and (4.221) can only be solved numerically. However, we make the following observations regarding its solution:

- For a fixed Doppler spread $B$ the tap spacing $\Delta \nu=B / f_{s} L$ when we have $L$ taps 
- $\tilde{P}(n)$ is increasingly ill-conditioned when $\Delta \nu$ decreases

- The eigenvalues of $\tilde{P}(n)$ determines the eigenvalues of the matrix $F=[I-$ $\left.\chi \tilde{P} Q_{0}\right] A$

- The term $2 \chi^{2} \tilde{P} \mathcal{E}^{2} \gamma P_{e} \operatorname{tr}\left(A \hat{\Pi} A^{H}\right) \tilde{P}^{H}$ of (4.221) is an order of magnitude smaller than the other terms involving $\hat{\Pi}$ because of its $\chi^{2}$ factor. If we neglect this term the solution of (4.221) is

$$
\hat{\Pi}(n)=F^{n} \hat{\Pi}(0)\left(F^{H}\right)^{n}+\sum_{m=0}^{n} F^{n} F_{0}\left(F^{H}\right)^{n}
$$

where

$$
F_{0}=\chi \tilde{P}\left(Q_{0}+Q_{e}\right) \tilde{P}^{H} \sigma_{w}^{2}+R+\chi^{2} \mathcal{E}^{2} \gamma P_{e} L \tilde{P} \tilde{P}^{H}
$$

provided that $F$ is stable

- The dimension of $F$ is $L \times L$ and its ill-conditioning is severe if $L \gg B \tau$ where $\tau=1 /\left((1-\lambda) f_{s}\right)$ is the time constant of the TU-RLS

The stability of $F$ depends on the conditioning of $\tilde{P}$. In addition, the TU-RLS depends on this conditioning because the algorithm estimates $\tilde{P}^{-1}(n)$ as discussed earlier in this section. Thus it is the eigenvalue spread of $\tilde{P}$ that limits the receiver stability in this case. The practical issue of when $\tilde{P}$ is close enough to singular to impose problems depends on, among other things, the receiver implementation. A rough rule [94] is that $\log _{10} \zeta$ decimal places are lost in the inversion of a matrix with condition number $\zeta$, therefore in practice the stability depends on the number of bits used in the receiver. A conservative rule is to require $\zeta<100$, and for any $\zeta$ the receiver becomes unstable when $L$ increases so that $\Delta \nu$ is small enough to render $\tilde{P}(n)$ singular for practical purposes. This is illustrated in Fig. 4-31 where we show the total channel estimation error variance, involving the numerical solution of 
(4.221), vs $\lambda$ and $L$ for a fixed Doppler spread. When $L$ increases and $\lambda$ decreases the condition number of $\tilde{P}$ increases as shown in Fig. 4-32. This is the reason for the increase in $\hat{\Pi}$ vs $L$. The theoretical result in Fig. 4-31 is verified by running the receiver on 15 trials of data as shown in Fig. 4-33. The SNR is $6 \mathrm{~dB}$, the Doppler spread is $5 \mathrm{~Hz}$ and the symbol rate is $2500 \mathrm{symb} / \mathrm{sec}$ in these figures. The flat area of the unstable regions of Fig. 4-33 and Fig. 4-31 is a plot artifact in order to show in more detail the structure of the stable region. The level in the transition region of the simulation Fig. 4-33 is slightly higher than the analytical result in Fig. 4-31. Two reasons for this may be the limited number of trials used in Fig. 4-33 and unmodeled behavior in the transition region. These figures illustrate the receiver limitation in terms of how many taps that can be used for a given SNR and Doppler spread.

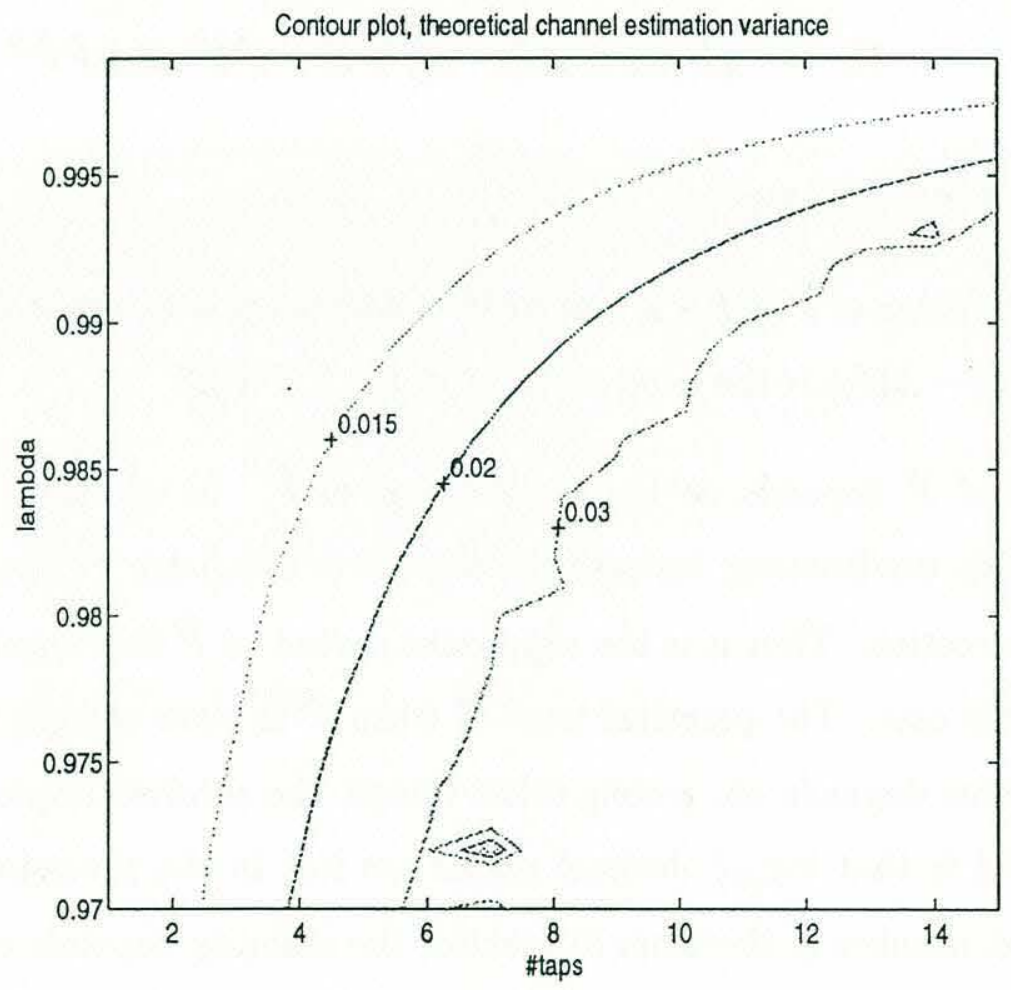

Figure 4-31: The theoretical total channel estimation error variance vs the number of taps $L$ and the forgetting factor $\lambda$. The increase of the variance for low $\lambda$ and high $L$ shows the receiver limitation due to ill conditioning of $\tilde{P}$. The Doppler spread is $5 \mathrm{~Hz}$ and the $\mathrm{SNR}$ is $6 \mathrm{~dB}$. 


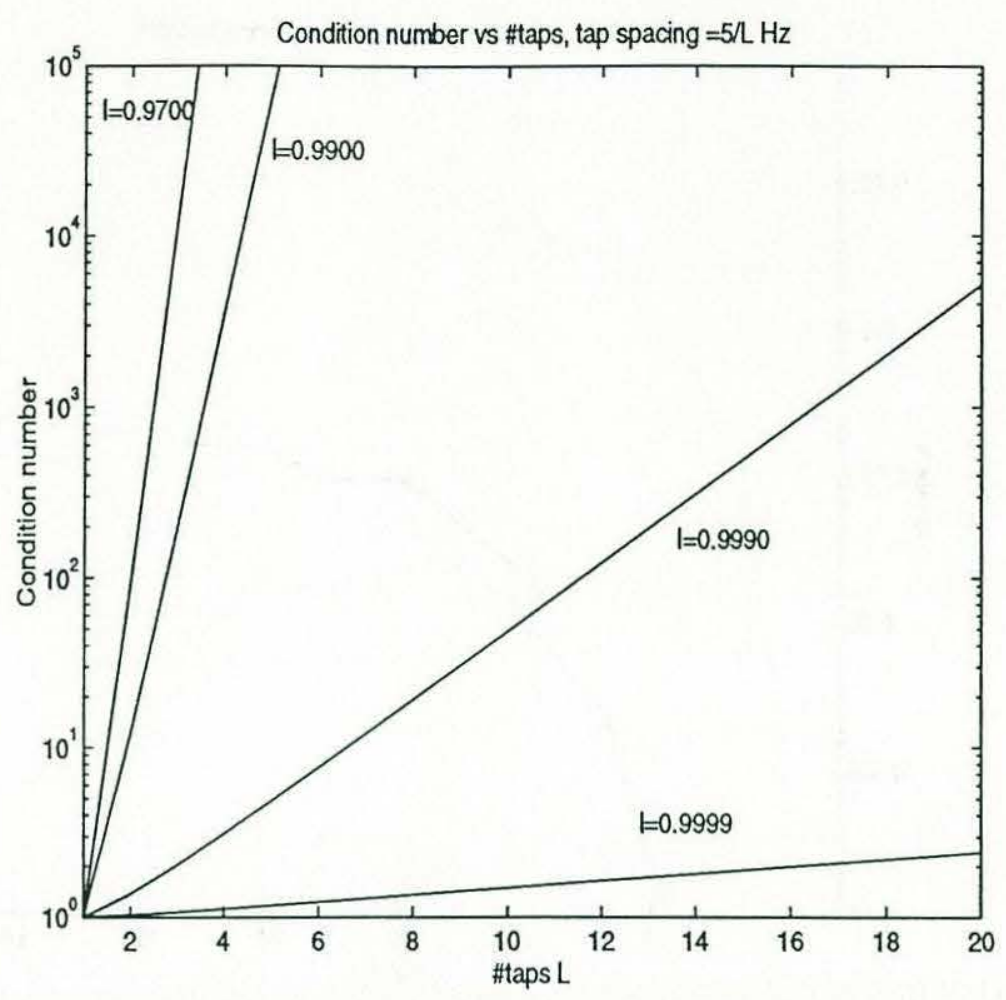

Figure 4-32: The condition number of $\tilde{P}$ vs the number of taps $L$ for different forgetting factors $\lambda$ and a Doppler spread of $5 \mathrm{~Hz}$ at a symbol rate of $2500 \mathrm{symb} / \mathrm{sec}$.

In order to further illustrate (4.221) we now show two examples. The first example is a purely Doppler spread channel, and the second example is a doubly spread channel with 3 taps where one of them is time-variant. This example serves as a more realistic ocean channel.

Example 1: Purely Doppler spread channel In this case we have one tap only in the channel impulse response

$$
h_{0}(n+1)=\alpha h_{0}(n)+v(n) .
$$

We choose $u_{0}^{2}=1$, so the channel model is

$$
y(n)=\sqrt{\mathcal{E}} h_{0}(n) z(n)+w(n)
$$




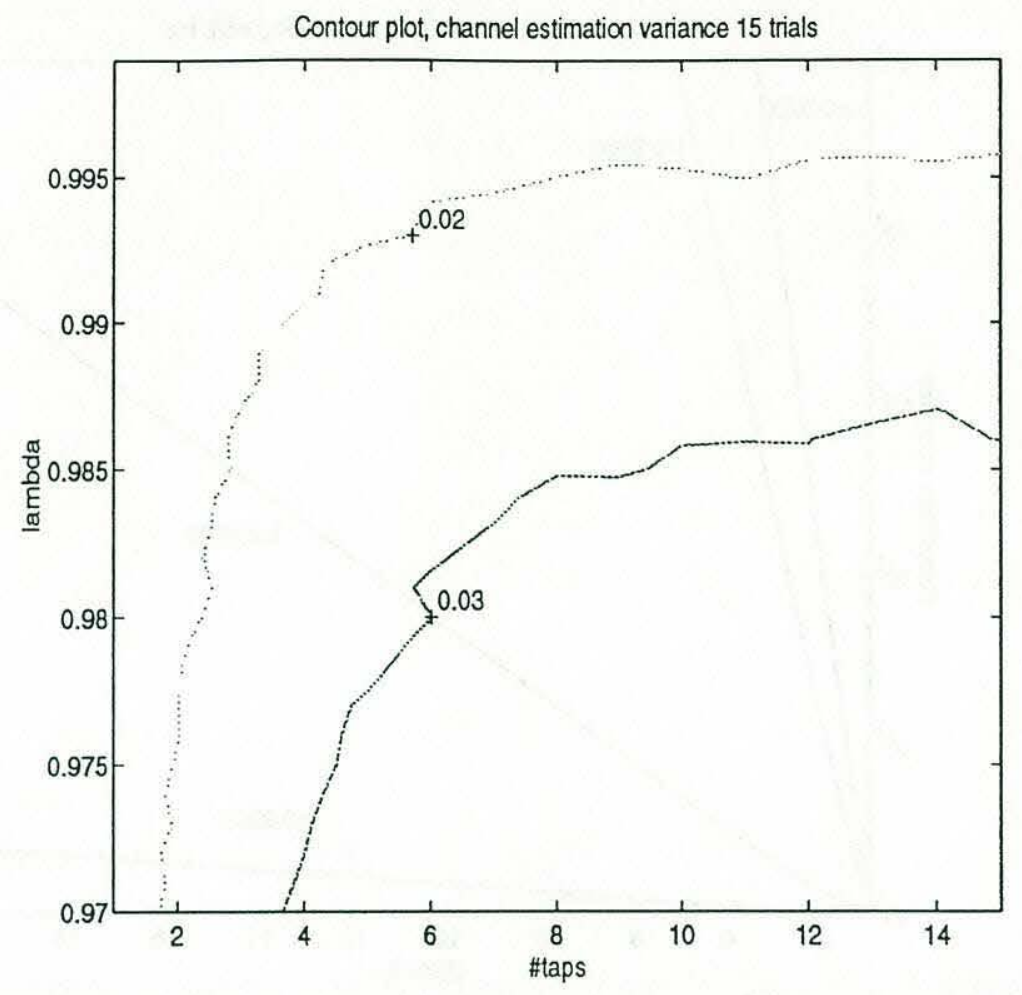

Figure 4-33: The total channel estimation error variance vs the number of taps $L$ and the forgetting factor $\lambda$ obtained by running the receiver on Doppler spread data. The increase of the variance for low $\lambda$ and high $L$ is consistent with the theoretical prediction, and it shows the receiver limitation due to ill conditioning of $\tilde{P}$.

$$
\tilde{R}_{y y}=R_{y y}=\mathcal{E}+\sigma_{w}^{2}
$$

We get from (4.200) and (4.88) that

$$
\begin{aligned}
J_{\min } & =\mathcal{E}\left(1-\frac{\mathcal{E}}{\sigma_{w}^{2}+\mathcal{E}}\right) \\
R & =1-\alpha^{2} \\
A & =\alpha .
\end{aligned}
$$

The channel variation is assumed to be an $\mathrm{AR}(1)$ process, and we have that $\alpha$ is given by (4.110) Fig. 4-34 shows the numerical solution for $P_{e}$ of (4.203), which involves the solution of (4.221), vs Doppler spread parameterized by the SNR. We remember 
that all the three effects of self noise, decision noise and lag noise are accounted for in (4.203), and they are all present in Fig. 4-34. At 3 dB SNR the self noise is masking the other noises, making the error almost equal over the whole Doppler range. At $10 \mathrm{~dB}$ SNR the self noise is low enough so that the lag noise becomes dominant at high Doppler spread, and at $15 \mathrm{~dB}$ SNR this effect is even more significant. The barely visible dotted lines show the probability of decoding error with no feedback errors present at the input to the channel tracker. This serves to show the effect of the decision error and it is negligible in this case. The dotted probability curves correspond to replacing the feedback of decoded symbols to the channel tracker in Fig. 4-20 with the correct symbol sequence.

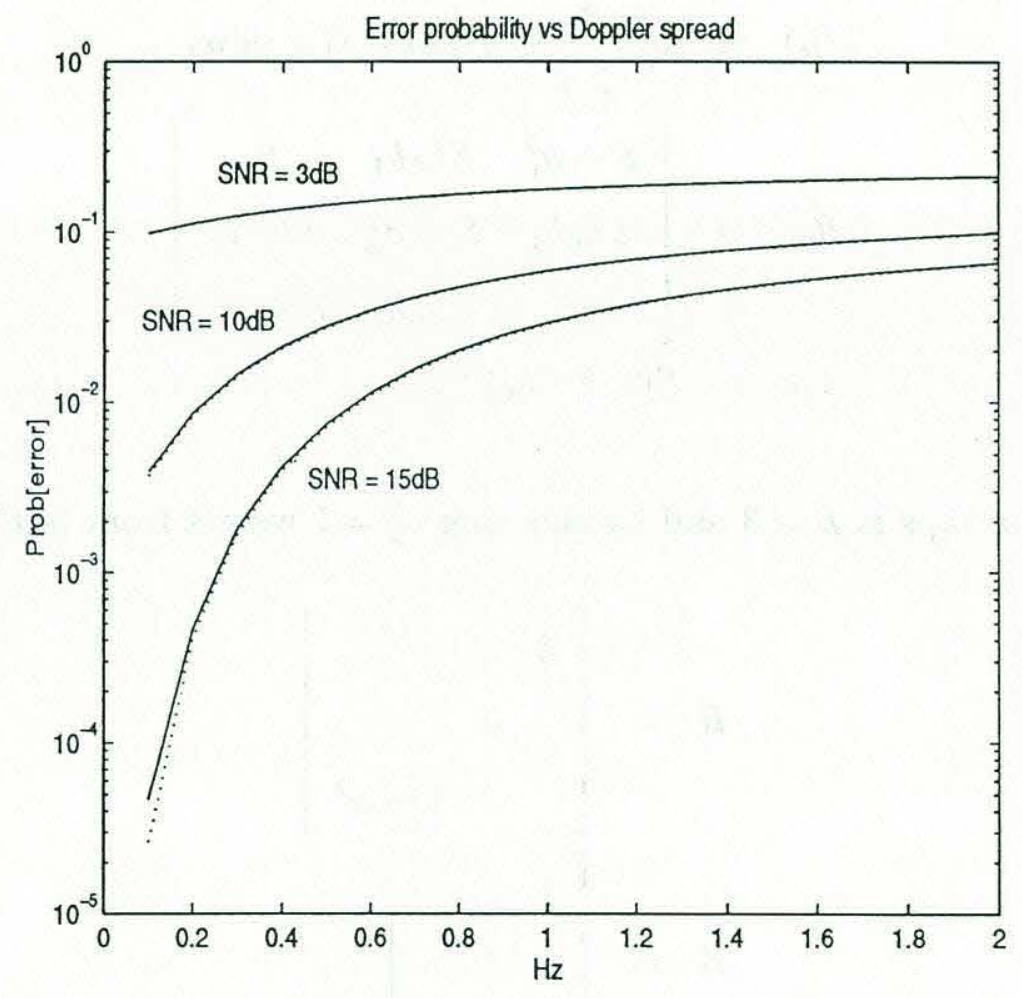

Figure 4-34: The probability of decoding error vs Doppler spread for a purely Doppler spread channel with the SNR as parameter. The symbol rate is assumed to be 600 symb/sec. The dotted lines are the probability of error when the decision errors are not fed back to the channel tracker. 
Example 2: Doubly spread channel We now assume the channel impulse response to contain the three taps

$$
\begin{aligned}
h_{0}(n) & =0.78 \\
h_{1}(n) & =0.62 \\
h_{2}(n+1) & =\alpha h_{2}(n)+v(n)
\end{aligned}
$$

where the taps are chosen to model a channel with impulse response corresponding to a direct path $h_{0}$, a bottom bounce $h_{1}$ and a surface bounce $h_{2}$. The channel model is

$$
\begin{aligned}
y(n) & =\sqrt{\mathcal{E}} \sum_{i=0}^{2} h_{i}(n) z(n-i)+w(n) \\
R_{y y} & =\left[\begin{array}{ccc}
\mathcal{E}+\sigma_{w}^{2} & \mathcal{E} h_{0} h_{1} & 0 \\
\mathcal{E} h_{0} h_{1} & \mathcal{E}+\sigma_{w}^{2} & \mathcal{E} h_{0} h_{1} \\
0 & \mathcal{E} h_{0} h_{1} & \mathcal{E}+\sigma_{w}^{2}
\end{array}\right] \\
\mathbf{r}_{z y} & =\mathcal{E}\left[\begin{array}{lll}
0 & h_{1} & h_{0}
\end{array}\right]^{H} .
\end{aligned}
$$

The number of taps is $L=3$ and by choosing $u_{2}^{2}=1$ we get from (4.88)

$$
\begin{aligned}
& R=\left[\begin{array}{lll}
0 & & \\
& 0 & \\
& & 1-\alpha^{2}
\end{array}\right] \\
& A=\left[\begin{array}{lll}
1 & & \\
& 1 & \\
& & \alpha
\end{array}\right] .
\end{aligned}
$$

By using (4.246), (4.110) and choosing $N=8$ in (4.200) we solve (4.203) numerically for different SNR and Doppler spreads. The result of this is shown in Fig. 4-35 and 
by comparing this to Fig. 4-34 we see that the error probability is now higher at a given Doppler spread for the high SNR case. The reason for this is twofold: (1) The minimum possible error variance $J_{\min }$ is higher for the delay spread channel of this example and (2) the self noise (4.236) and the decision noise (4.237) are increasing because of more taps. This causes $\sigma_{e}^{2}\left(P_{e}\right)$ in (4.203) to be higher at a given SNR and it explains some of the difference between the two examples. In this more realistic channel the decision noise is contributing significantly, and we can see the increase in error probability as the difference between the dotted and solid curves in Fig. 4-35.

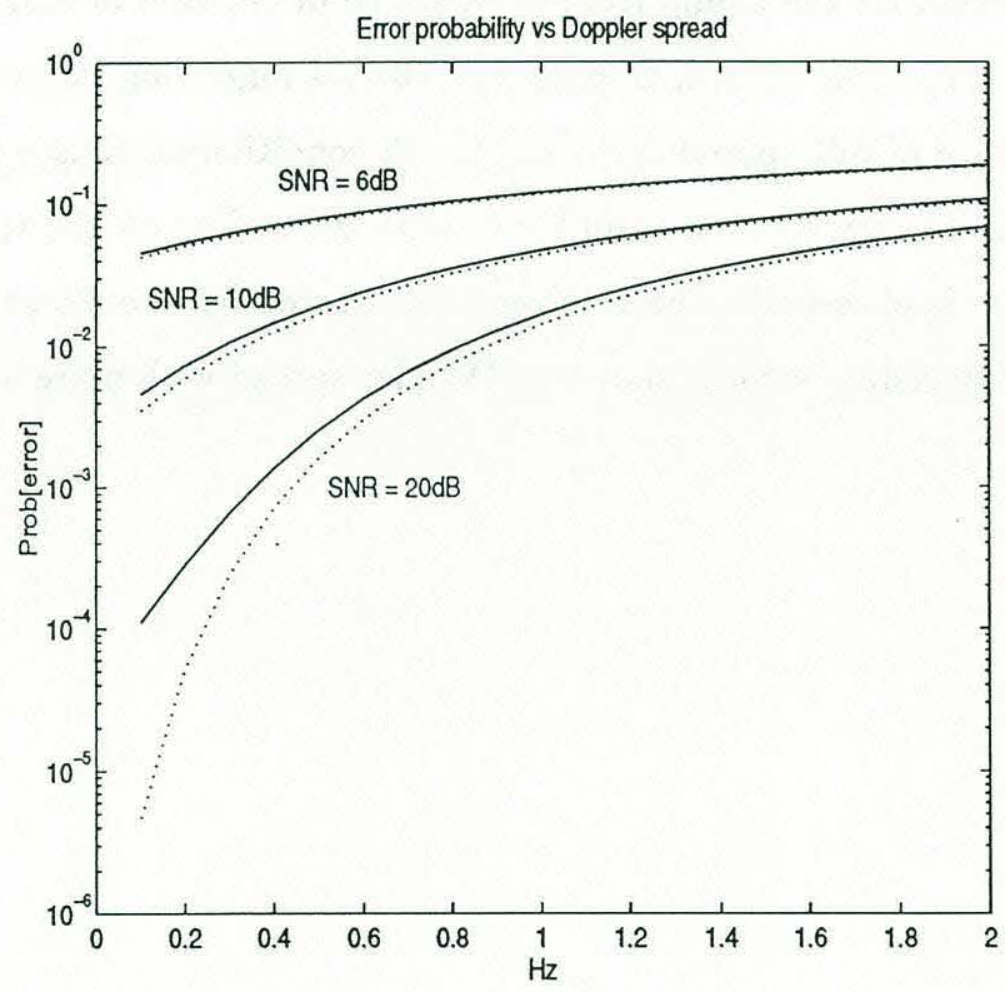

Figure 4-35: The probability of decoding error vs Doppler spread for a doubly spread channel with the SNR as parameter. The symbol rate is assumed to be $600 \mathrm{symb} / \mathrm{sec}$ and the delay spread is $5 \mathrm{msec}$. The dotted lines are the probability of error when the decision errors are not fed back to the channel tracker. 
Summary The receiver operates in presence of both initialization errors and data corrupted by noise, delay spread and Doppler spread. We show that the result of Doppler initialization error is tap attenuation in the TU-RLS channel tracker. The errors in the feedback of symbols used in the channel tracker causes noise in the channel estimate, and we find an equilibrium where the probability of decoding error is consistent with the noise, delay spread and Doppler spread in the data. The stability is determined by the conditioning of $\tilde{P}(n)$ and we treat the two extremes of diagonal and fully populated $\tilde{P}(n)$, noting that the block diagonal structure of $\tilde{P}(n)$ in the general case motivates that these two cases are the most important ones. We find the condition for the equilibrium to be stable in the case of diagonal $\tilde{P}$, and it is given by noise, delay spread, Doppler spread and forgetting factor $\lambda$ of the TURLS. In the case of fully populated $\tilde{P}(n)$ the ill conditioning causes the receiver to become unstable when a certain number of taps, depending on the receiver number representation, is exceeded. The receiver is illustrated by two examples where the probability of decoding error is shown vs Doppler spread with noise as a parameter. 


\subsection{Verification of the receiver}

Preview The receiver structure in Fig. 4-20, called the TU-RLS in the following, is tried on both simulated and real data in order to demonstrate its capabilities and limitations. In Section 4.4.1 we treat the case when several rays with different Doppler shifts contribute to the received signal so that the Doppler spread contains several discrete frequencies at different delays. In this case the receiver in (4.92) is applicable, and results are shown in Fig. 4-36-4-38. In Section 4.4 .2 we treat the case when the relative ray arrival times are less than one symbol interval. Thus the Doppler spread is made up of several frequencies at the same delay, and the receivers from Section 4.3.4 are used to achieve the results in Fig. 4-40-4-43. If the Doppler spread contains a continuous frequency band, which may be the case for a time-variant ray reflected from the ocean-surface, we use the IFS based receiver (4.120) with the recursive channel tracker 4.109 and the result is shown in Fig. 4-47. In Section 4.4.3 real data decoding from the Gould Island experiment described in Section 3.2.4 is shown in Fig. 4-51 for channels without significant Doppler spread and in Fig. 4-52-4-54 for three severely Doppler spread channels.

\subsubsection{One Doppler shift for each delay}

We simulate scenarios where the channel response is constructed from several rays with different Doppler shifts. This is discussed in Chapter 3 and it occurs as a result of transmitter or receiver relative motion, and also when some rays interacts with an ocean-surface that has a long swell.

Common parameters For all figures in this section except Fig. 4-39 $3 \mathrm{~dB}$ contours of the ambiguity function (2.32) are shown in the upper left panel, complex

values of the TU-RLS taps $\hat{\mathrm{h}}(n)$ as given by (4.92) in the upper right panel and the estimates of the decoded symbols $\hat{z}(n)$ from (4.138) in the lower left panel. The sym- 
bol rate is $2500 \mathrm{symb} / \mathrm{sec}$ in all simulations of this section and the SNR is $15 \mathrm{~dB}$. The channel tracker in (4.92) and (4.93) is started with $\hat{\mathbf{h}}(0)=0$ and $P(0)=100 I$, and the training sequence duration is 512 symbols. The training sequence is used both to compute the cross-ambiguity function and to achieve initial convergence of the TU-RLS. The tap initialization is carried out by thresholding the cross-ambiguity function. In some of the figures parameters are listed in the lower right panel. The "\#taps, tracking" is the channel tracker dimension $L$ of (4.83), the "\#taps, inversion" is the FIR filter order $L_{1}$ of $(4.138)$, the "SNR" is the ratio $\mathcal{E} / \sigma_{w}^{2}$, "lambda" is the exponential weighting factor $\lambda$ of the TU-RLS (4.93) and "\# errors in" is the ratio of transmitted to erroneously decoded symbols.

Fig. 4-36 shows the result of a channel where the signal is arriving over three rays with slightly different delays and different Doppler shifts. The delays and Doppler shifts are

$$
\begin{aligned}
& \mathrm{n}=\left[\begin{array}{lll}
0 & 1 & 2
\end{array}\right] \text { symbols }=\left[\begin{array}{lll}
8 & 8.4 & 8.8
\end{array}\right] \mathrm{msec} \\
& \boldsymbol{\nu}=\left[\begin{array}{lll}
4 & 0 & -4
\end{array}\right] \mathrm{Hz} .
\end{aligned}
$$

It demonstrates the ability to decode in presence of Doppler spread that contains several discrete tones. The circular patterns traced by the tap magnitudes in the upper right panel of Fig. 4-36 shows the fact that the channel tap magnitudes are constant. The channel is time variant only because each tap has a fixed Doppler shift. The complex tap values change because of this, but since their magnitudes are constant they stay on a circle in the complex plane. Therefore the tracking of the taps also stay on circles, and this is observed in the upper right panel of Fig. 4-36. The time between adjacent tick marks on the circles is $250 \mathrm{msec}$.

Fig. 4-37 shows the limitation of the linear signal combiner to decode in the presence of delay spread. The reason for the much noisier symbol estimation is seen in the ambiguity function which shows that the communication channel has two 
almost equal amplitude lags around 8 and $10 \mathrm{msec}$. The channel delays and Doppler shifts are

$$
\begin{aligned}
& \mathrm{n}=\left[\begin{array}{lllll}
0 & 1 & 2 & 3 & 4
\end{array}\right] \text { symbols }=\left[\begin{array}{lllll}
8 & 8.4 & 8.8 & 9.2 & 9.6
\end{array}\right] \mathrm{msec} \\
& \boldsymbol{\nu}=\left[\begin{array}{lllll}
4 & 0 & 8 & 0 & 0
\end{array}\right] \mathrm{Hz} \text {. }
\end{aligned}
$$

When the SNR is high the decoder (4.138) attempts to invert the channel response, and it is constrained to be a FIR filter. The inversion of a channel response with almost equal taps leads to a high order FIR filter, and we explain this in more detail in Section 4.4.2.

Fig. 4-38 shows an example of a sparse communication channel with different Doppler shifts on the two widely spaced returns The channel parameters are

$$
\begin{aligned}
& \mathrm{n}=\left[\begin{array}{ll}
0 & 50
\end{array}\right] \text { symbols }=\left[\begin{array}{ll}
8 & 28
\end{array}\right] \mathrm{msec} \\
& \boldsymbol{\nu}=\left[\begin{array}{ll}
0 & 4
\end{array}\right] \mathrm{Hz} .
\end{aligned}
$$

The TU-RLS contains only two taps and the estimated symbols are based on a signal combiner with 8 taps. This scenario is the result when one direct path and one surface reflected path are present, and the ocean-surface has a swell with period significantly longer than the packet length.

The adaptive DFE with a PLL that is discussed in Section 4.2 is unable to decode any of the cases shown in Fig. 4-36, Fig. 4-37 and Fig. 4-38 because the total Doppler spread is too large. The case where this receiver achieves its best performance is for the cross-ambiguity function shown in Fig. 4-38. This signal is made from a channel with one direct ray and one surface reflected ray with a slow swell on the surface that gives the Doppler shift shown. If there is no swell on the surface the channel is LTI, and the DFE decodes correctly as shown in the upper left panel of Fig. 4-39. The right column of this figure shows the predicted symbols for channels where the 
first return is at $0 \mathrm{~Hz}$ as in Fig. 4-38 and the second return is at 0,1 and $2 \mathrm{~Hz}$ in the upper, middle and lower panels respectively. The left column shows the mean square error in the predictions as computed by

$$
J(n)=\frac{1}{N} \sum_{k=n}^{n+N-1}|\hat{z}(k)-\tilde{z}(k)|^{2}
$$

which is a moving average estimate of the power in the decision error that is used as input to the RLS. As can be seen from Fig. 4-39 it is found that the receiver can decode in the presence of a Doppler difference of $1 \mathrm{~Hz}$ between the two paths, but that it is unable to decode when the difference is $2 \mathrm{~Hz}$.

Summary We demonstrate the capability of the receiver presented in Section 4.3.3. Fig. 4-36 shows the decoding of a scenario corresponding to different Doppler shifts on rays arriving at different times. Fig. 4-37 demonstrates the result of the constraint that the decoder is linear, and Fig. 4-38 shows decoding of a sparse channel. We compare with results from the DFE in Fig. 4-39. 

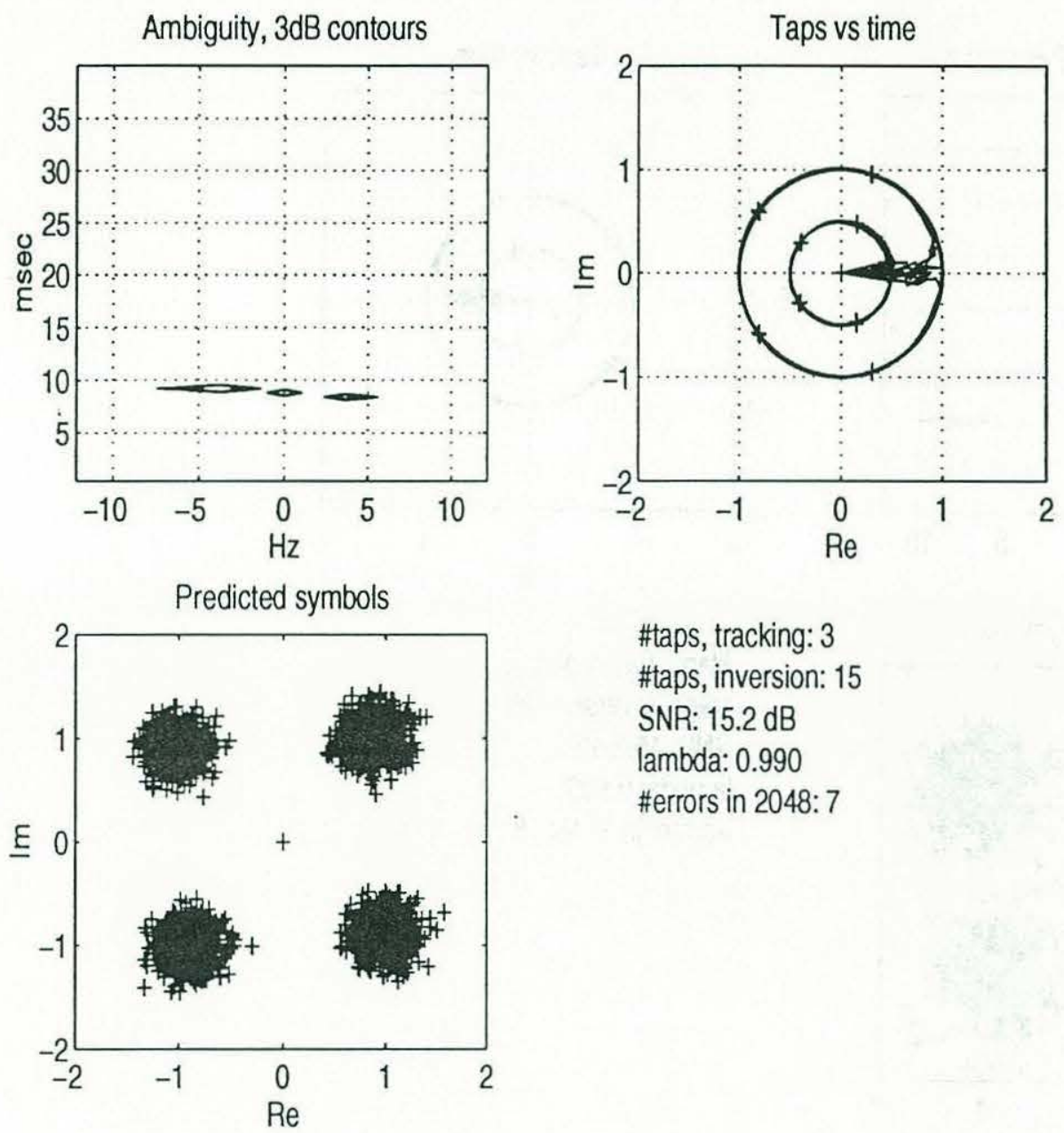

\#taps, tracking: 3

\#taps, inversion: 15

SNR: $15.2 \mathrm{~dB}$

lambda: 0.990

\#errors in 2048: 7

Figure 4-36: Decoding of Doppler spread data, the ambiguity function shows three returns at different Doppler shifts (upper left panel), the TU-RLS taps are shown to rotate at different amplitudes (upper right panel), and the eye pattern showing the decoding result is open (lower left panel). 

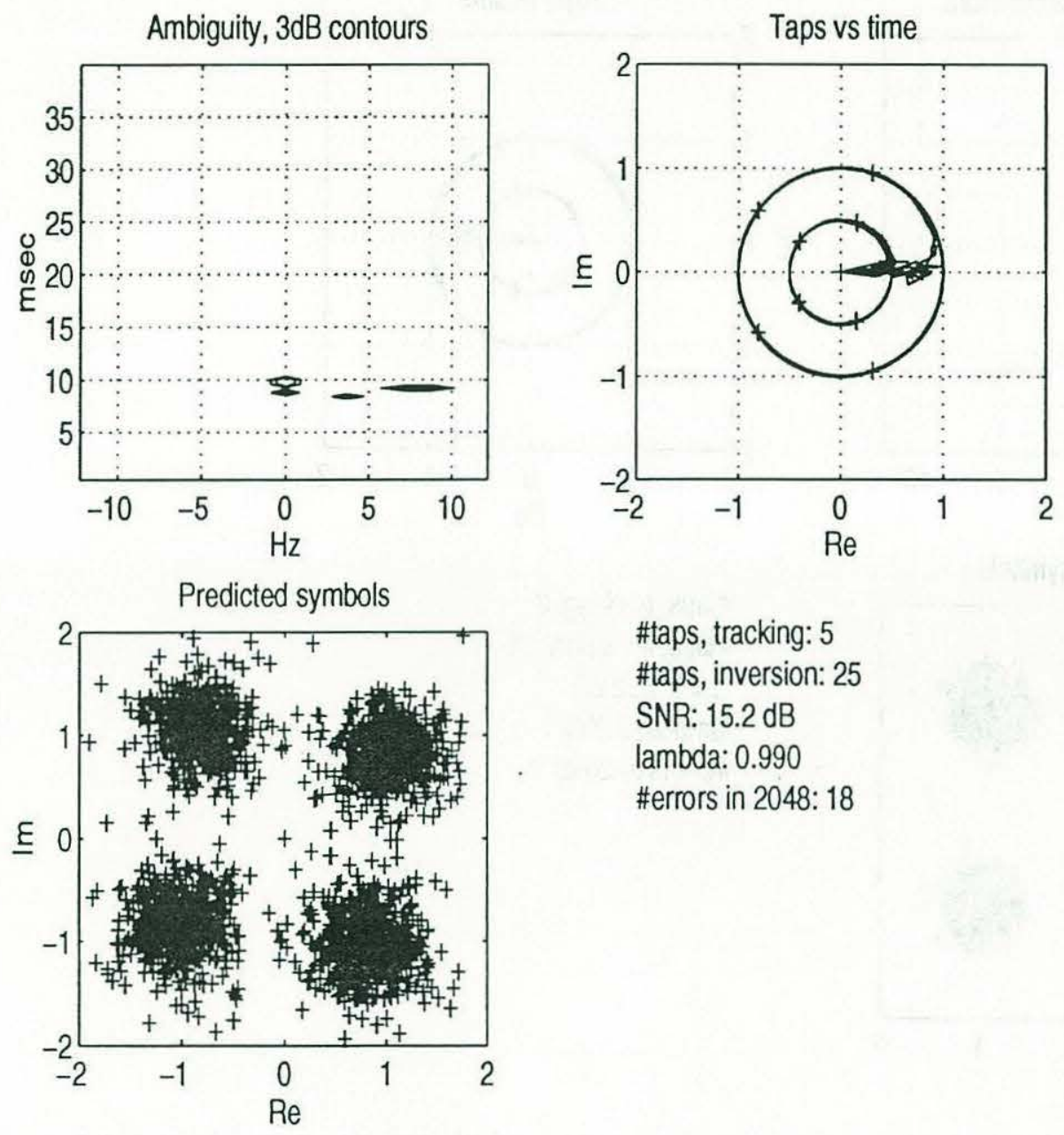

\#taps, tracking: 5

\#taps, inversion: 25

SNR: $15.2 \mathrm{~dB}$

lambda: 0.990

\#errors in 2048: 18

Figure 4-37: Decoding of doubly spread data where the FIR signal combiner has more difficulty because there are returns of almost equal amplitude at different delays. 

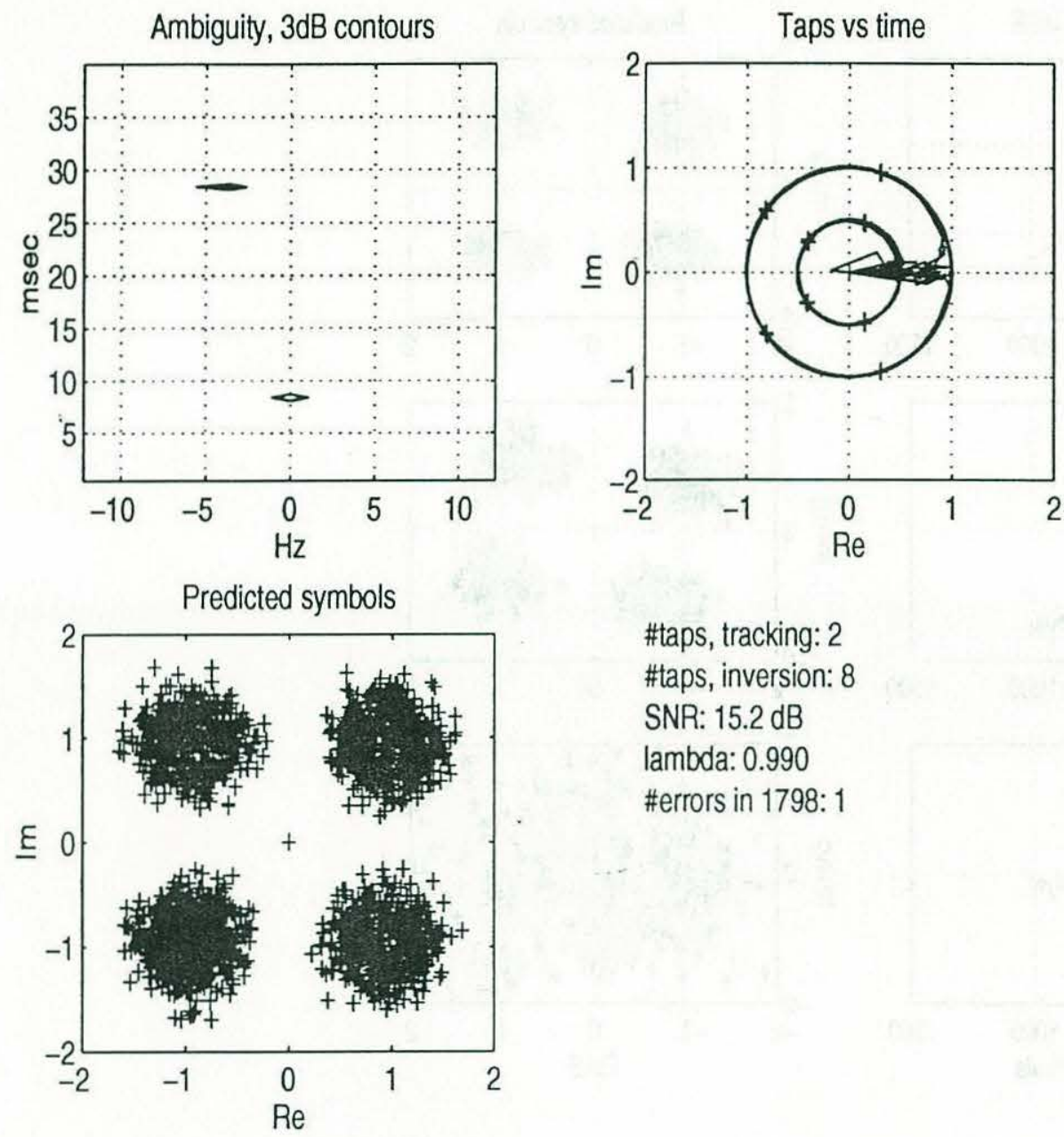

\#taps, tracking: 2

\#taps, inversion: 8

SNR: $15.2 \mathrm{~dB}$

lambda: 0.990

\#errors in 1798: 1

Figure 4-38: Decoding of a typical sparse underwater communication channel with many symbol intervals between the returns and the returns at different Doppler shifts. The second return is from a surface swell with much longer period than the packet length, so that a Doppler shift rather than a spread is the result. 

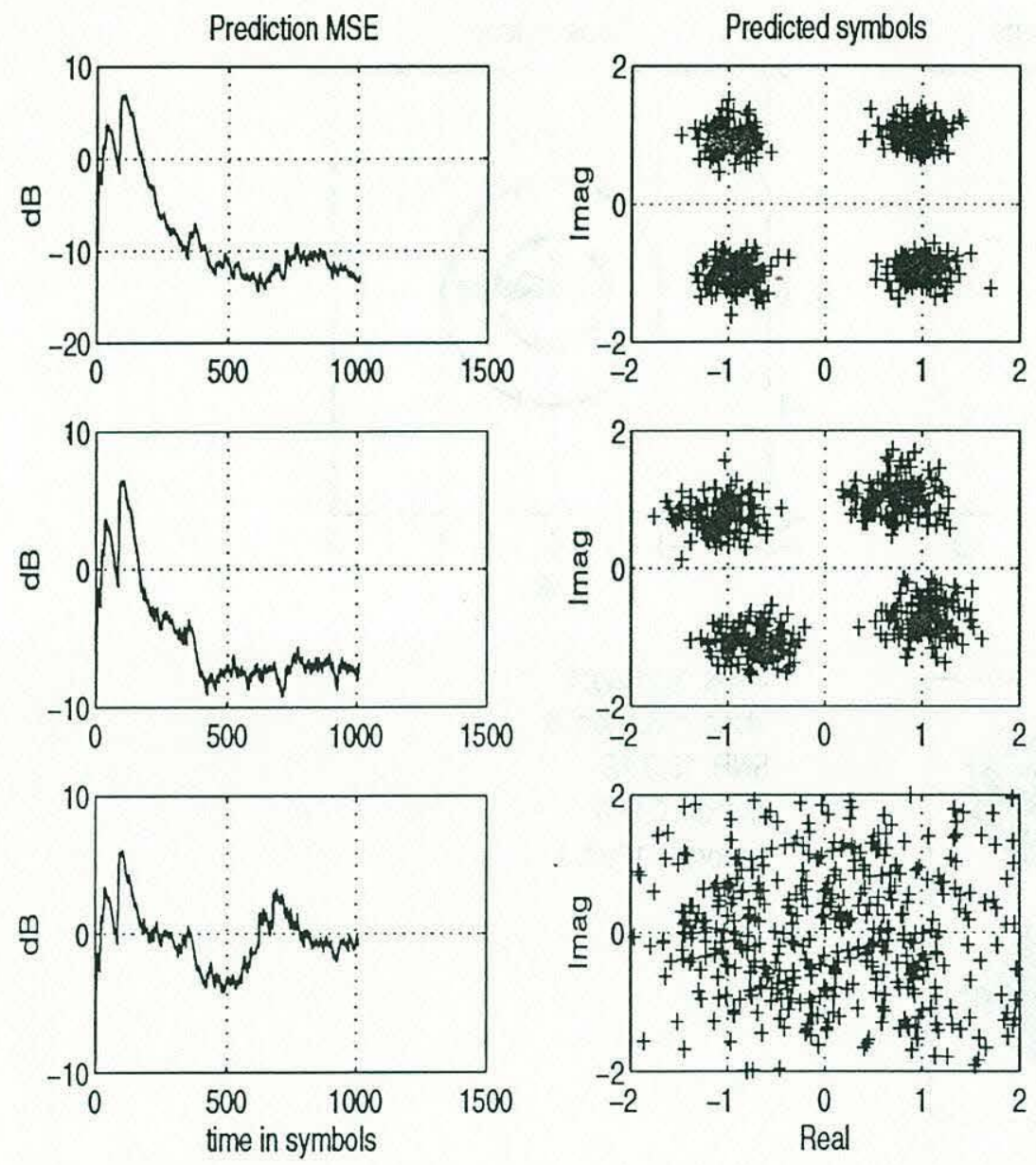

Figure 4-39: Performance of the DFE receiver with a PLL on a Doppler spread signal. The predicted symbols in the right column and the MSE decision error in the right column for a two path signal with respectively 0,1 and $2 \mathrm{~Hz}$ difference in Doppler between the paths in the upper, middle and lower panels. 


\subsubsection{Multiple Doppler shifts for each delay}

In the simulations and real data presented in Chapter 3 we find that different rays may arrive the receiver in the same symbol interval. If these rays have different Doppler shifts we have a scenario with discrete frequencies at the same delay so that the composite signal is Doppler spread. Depending on the surface roughness, as discussed in Chapter 3, a surface reflected ray may also have a continuous Doppler spread. We simulate both discrete and continuous Doppler spread.

Common parameters The upper and lower left panels of all figures in this chapter shows the cross-ambiguity function and the decoding result; see Section 4.4.1 for more description and references. The initialization of the taps is carried out by thresholding the cross-ambiguity function based on 512 symbols, and the symbol rate is $2500 \mathrm{symb} / \mathrm{sec}$ in all the examples of Section 4.4.2. The lower left panel shows the decoding result. The information in the lower right panel of the figures in this section is described in Section 4.4.1.

\section{Discrete Doppler spread}

The Doppler line based receiver that we derive in Section 4.3.4 is used on purely Doppler spread data, and an example of decoding with the IFS Doppler line is shown in Fig. 4-40 where the cross-ambiguity function of the Doppler spread channel is in the upper left panel. It has 4 Doppler shifts and amplitudes at

$$
\begin{aligned}
\nu & =\left[\begin{array}{llll}
-1 & 1 & 4 & 8
\end{array}\right] \mathrm{Hz} \\
\mathrm{U}(0) & =\left[\begin{array}{llll}
.5 & 1 & 1 & .8
\end{array}\right]^{T}
\end{aligned}
$$

where the amplitudes are evolving according to

$$
\mathbf{U}(n)=\operatorname{diag}(\boldsymbol{\alpha}) \mathbf{U}(n-1)
$$




$$
\alpha=\left[\begin{array}{llll}
1.0001 & 1 & .9999 & .9995
\end{array}\right]
$$

We note that this implies a different channel model than the AR(1) (4.86) for which the receiver is derived, and the example illustrates tracking of slowly varying rays with constant Doppler shifts. The recursive estimator (4.109) and (4.93) for the channel tracker is used, and the upper right panel shows in absolute value the convergence of the channel tracker taps to the right coefficients when they are started from $\hat{\mathbf{h}}(0)=\mathbf{0}$. The first 256 values of the tap magnitudes in the upper right panel of Fig. 4-40 is the training of the TU-RLS. The tap frequency locations are found from thresholding the cross-ambiguity function as described in Section 4.3.6, therefore they are not perfectly matched to the values in (4.251). The tap values obtained by using the initialization rule in Section 4.3 .6 are

$$
\hat{\boldsymbol{\nu}}=\left[\begin{array}{llll}
-1.1 & 1.04 & 4.1 & 7.9
\end{array}\right] \mathrm{Hz} .
$$

The scenario of Fig. 4-40 corresponds to distinct rays with different Doppler shifts arriving within the same symbol interval. The receiver used in Fig. 4-40 is an IFS Doppler line, as given by (4.120), that is updated with the recursive TU-RLS channel tracker.

We show the decoding of a channel with

$$
\begin{aligned}
& \boldsymbol{\nu}=\left[\begin{array}{ll}
0 & 3
\end{array}\right] \mathrm{Hz} \\
& \mathbf{h}=\left[\begin{array}{ll}
1 & .9
\end{array}\right]^{T} .
\end{aligned}
$$

in Fig. 4-41 and

$$
\begin{aligned}
\nu & =\left[\begin{array}{llll}
0 & 1 & 2 & 3
\end{array}\right] \mathrm{Hz} \\
\mathrm{h} & =\left[\begin{array}{llll}
1 & .8 & .7 & .6
\end{array}\right]^{T} .
\end{aligned}
$$


in Fig. 4-42. These results, as will be evident when we compare them to the results of the FFS receiver later in this chapter, illustrate the noise enhancement associated with an IFS receiver, and we use the block processing (4.107) to estimate the channel in these examples.

We motivate and show in Section 4.3.4 that also the FFS Doppler line is useful for Doppler spread data, and we now use a receiver with the FFS Doppler line. In order to show how this receiver works in presence of Doppler spread we present two examples using the channels of Fig. 4-41 and Fig. 4-42. In this case the receiver is a single FFS Doppler line, and it is given by (4.131). The examples in this section use the block channel tracker (4.107), and the block estimate of the FFS coefficients is shown in the upper right panel of Fig. 4-43 and Fig. 4-45 as opposed to the time evolution of the Doppler line coefficients that is shown in Fig. 4-40.

The first example in Fig. 4-43 where the channel is given by (4.254) shows an open eye over the symbols that are transmitted. The number of coefficients used is 20 which is a large number compared to the 2 frequencies actually present in the simulated data. The reason for this is that the relative amplitudes of these 2 frequencies are almost equal so that many taps are needed in the FFS Doppler line in order to compensate this, and we now explain this by using duality. The dual scenario is when a FIR filter is used to equalize a delay spread channel with frequency response

$$
H(z)=1+\alpha z^{-1} .
$$

The zero forcing (IIR) equalizer for this channel is $1 / H(z)$, and when we use a FIR filter as equalizer its coefficients are determined by the best possible approximation to this transfer function which is

$$
\frac{1}{H(z)}=1-\alpha z^{-1}+\alpha^{2} z^{-2}-\cdots
$$


If $\alpha$ is close to 1 , which corresponds to the case of having almost equal frequency amplitudes as shown in the cross-ambiguity function of Fig. 4-43, we need many terms of the sum (4.257) in order to approximate the zero forcing equalizer.

The more complex Doppler spread of (4.255) is shown in Fig. 4-45. The decoding in the lower left panel shows that the receiver works well on this relatively complex channel.

The tap configuration in Fig. 4-43 shows adjacent taps with nearly equal magnitude and opposite sign. This is reminiscent of super directivity as discussed in Section 4.3.4, and we use the constrained least squares fit (4.136) in Fig. 4-44 in order to avoid this behavior. The upper right panel of Fig. 4-45 also shows adjacent coefficients of opposite sign and comparable magnitude. We show the constrained Doppler line solution (4.136) in Fig. 4-46 for the same channel as in Fig. 4-45. In both Fig. 4-44 and Fig. 4-46 the Doppler line is constrained to have unity white noise gain as given by (4.133), and we see the impact of this modification by comparing them to the upper right panels of Fig. 4-43 and Fig. 4-45. The more sensitive receivers of Fig. 4-43 and Fig. 4-45 are replaced with the more robust receivers Fig. 4-44 and Fig. 4-46 at the expense of higher error rates as seen in the lower left panels of these figures. The eye pattern in Fig. 4-44 shows significant deviation from the unconstrained Fig. 4-43. This is due to the strength of the constraint we use, and a tradeoff exists between the strength of the constraint used to solve (4.135) and the resulting modification of the eye pattern.

By comparing Fig. 4-42 with Fig. 4-45 and Fig. 4-41 with Fig. 4-43 we observe the noise enhancement effect as the lower number of errors with the FFS receiver for these two channels. 

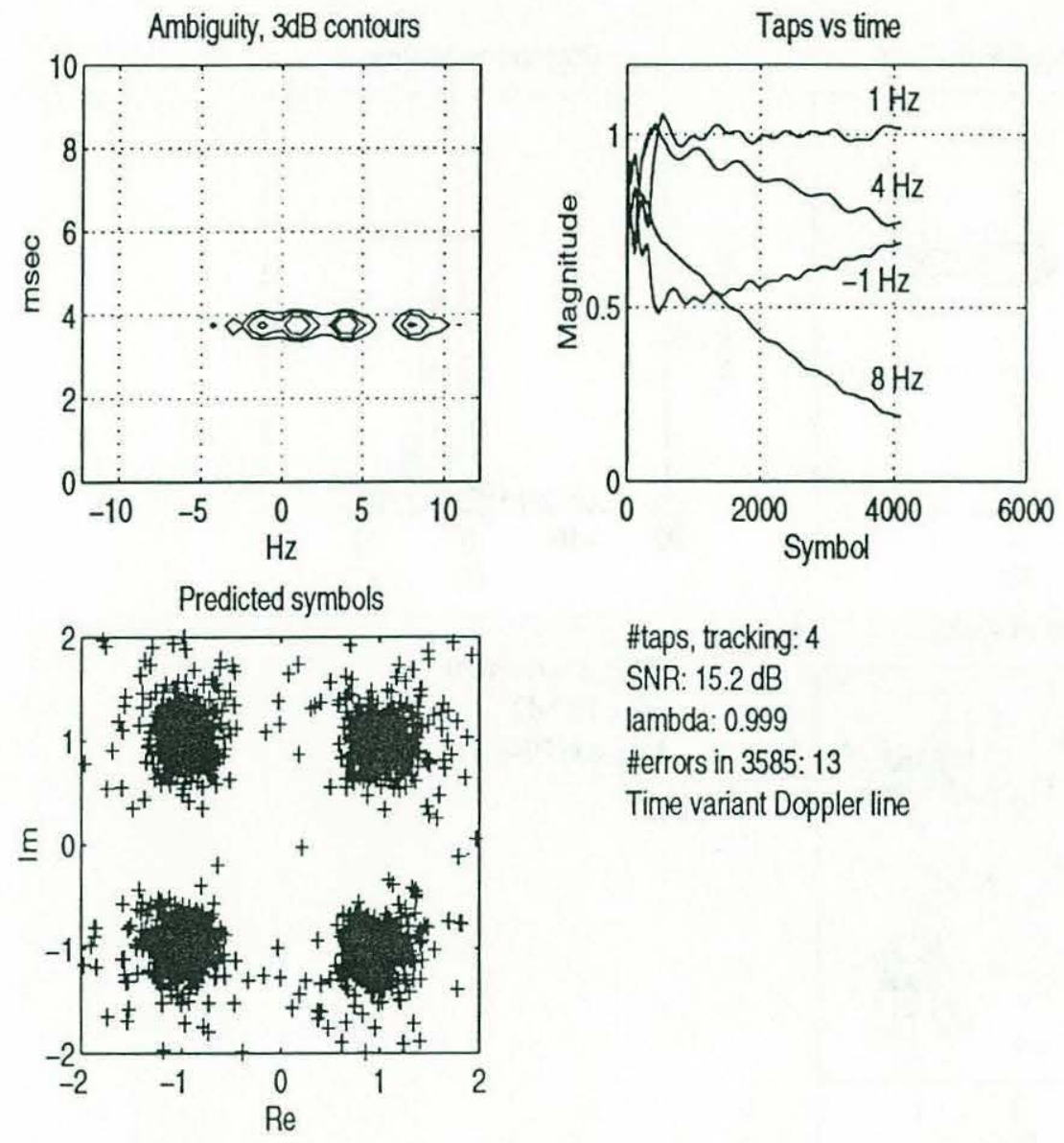

Figure 4-40: Decoding using the IFS Doppler line and recursive channel tracker. The tap locations are found by thresholding the cross-ambiguity function in the upper left panel. The initial convergence of the TU-RLS from training data is shown in the first 256 symbols in the upper right panel. 

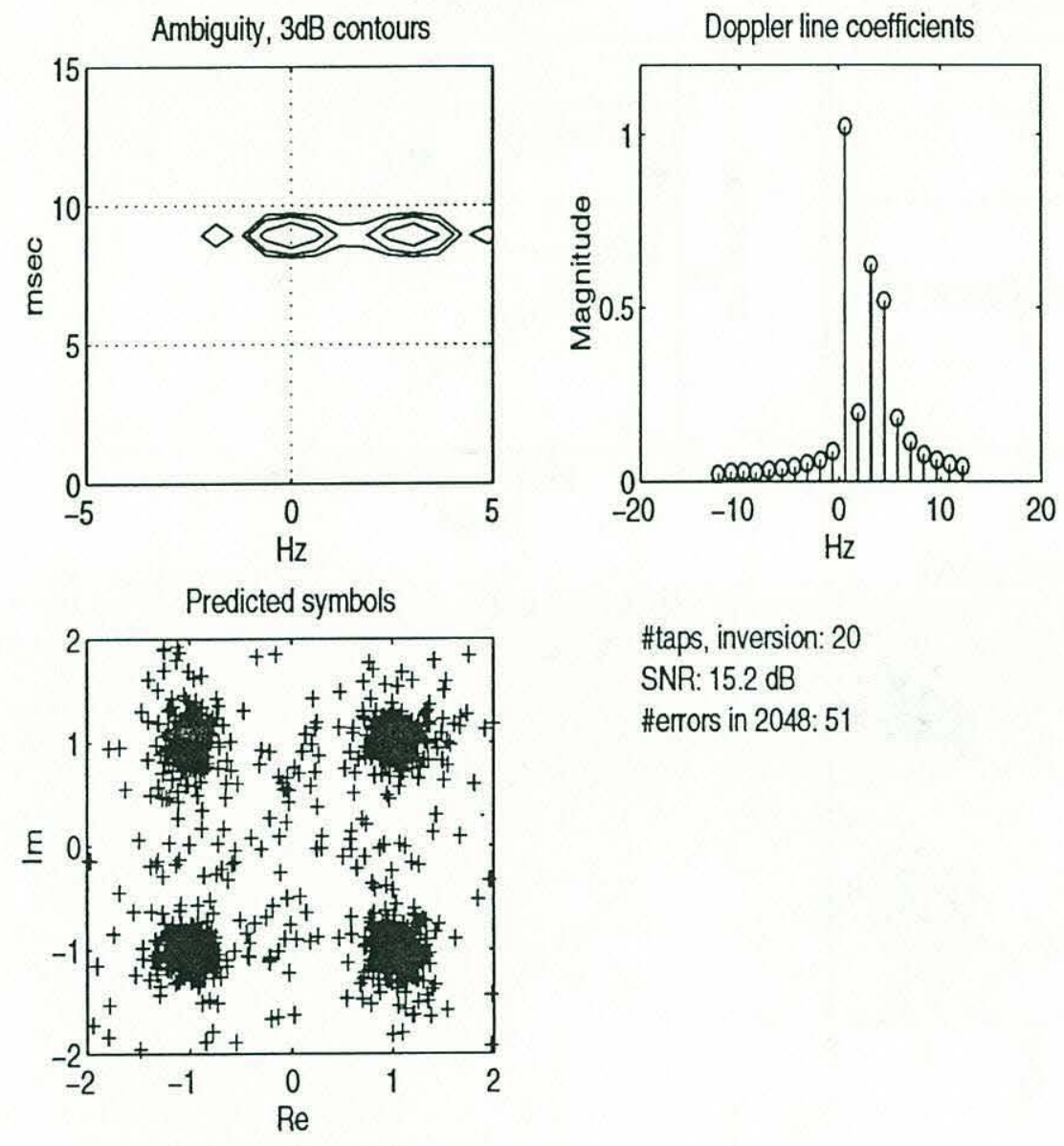

\#taps, inversion: 20

SNR: $15.2 \mathrm{~dB}$

\#errors in 2048: 51

Figure 4-41: Decoding using the IFS Doppler lines and the block channel estimator. There are two rays with different frequencies. 

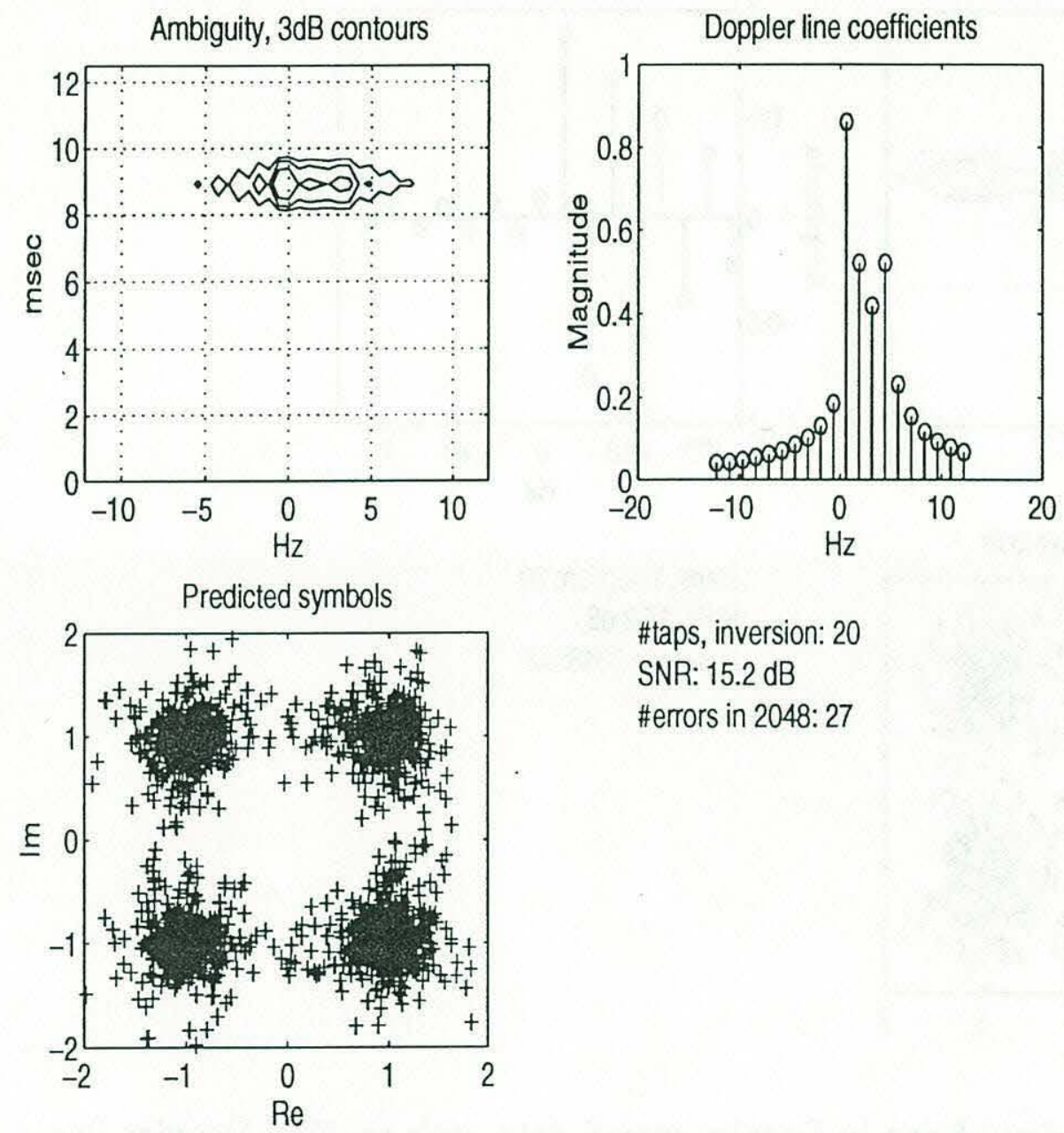

\#taps, inversion: 20

SNR: $15.2 \mathrm{~dB}$

\#errors in 2048: 27

Figure 4-42: Decoding using the IFS Doppler lines and the block channel estimator. There are four rays with different frequencies. 

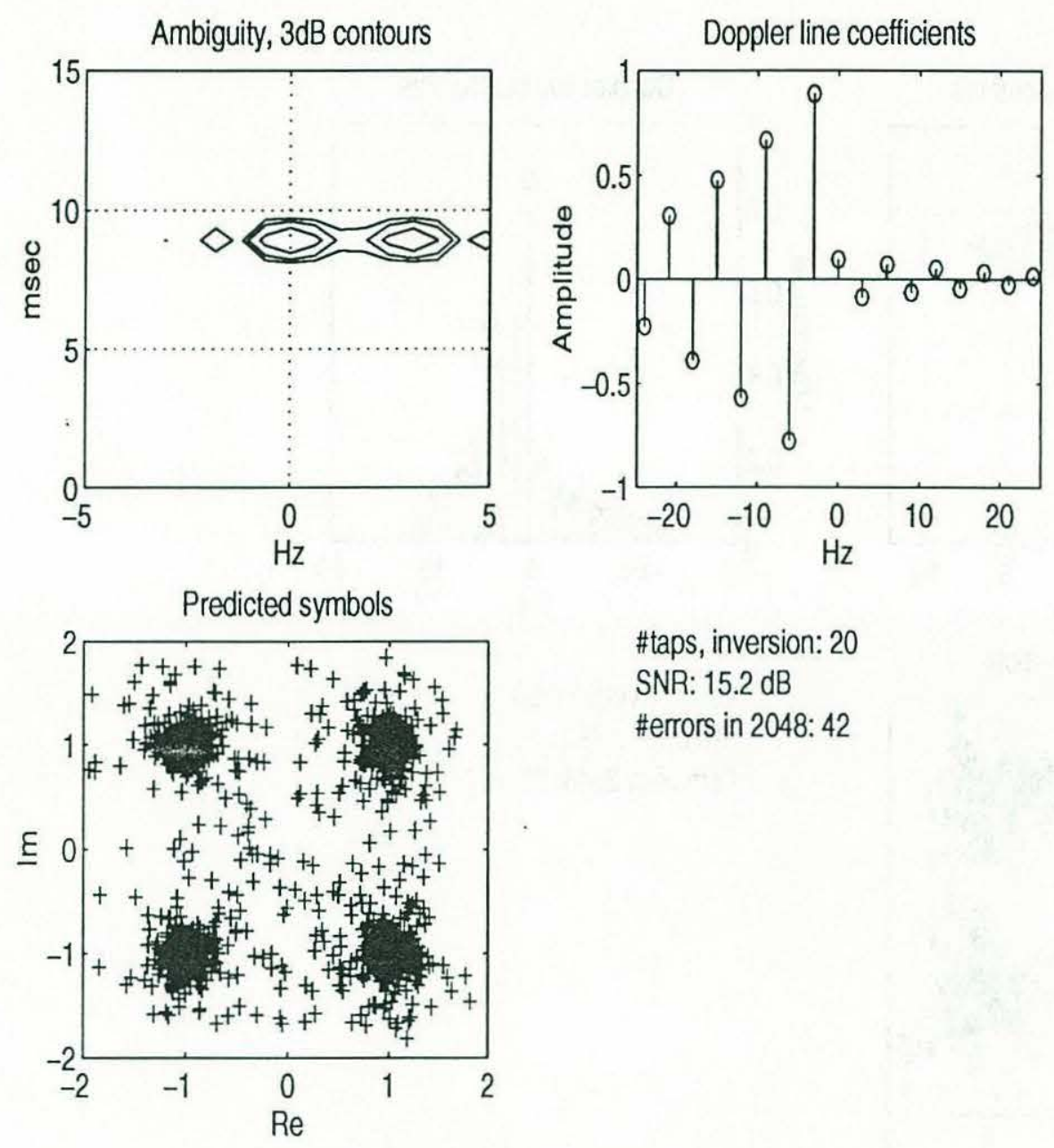

\#taps, inversion: 20

SNR: $15.2 \mathrm{~dB}$

\#errors in 2048: 42

Figure 4-43: Decoding of purely Doppler spread data with an FFS Doppler line. There are 2 frequencies in the received data. The channel cross-ambiguity function in upper left panel, the FFS coefficients in upper right panel and the decoded symbol estimates in the lower left panel. 

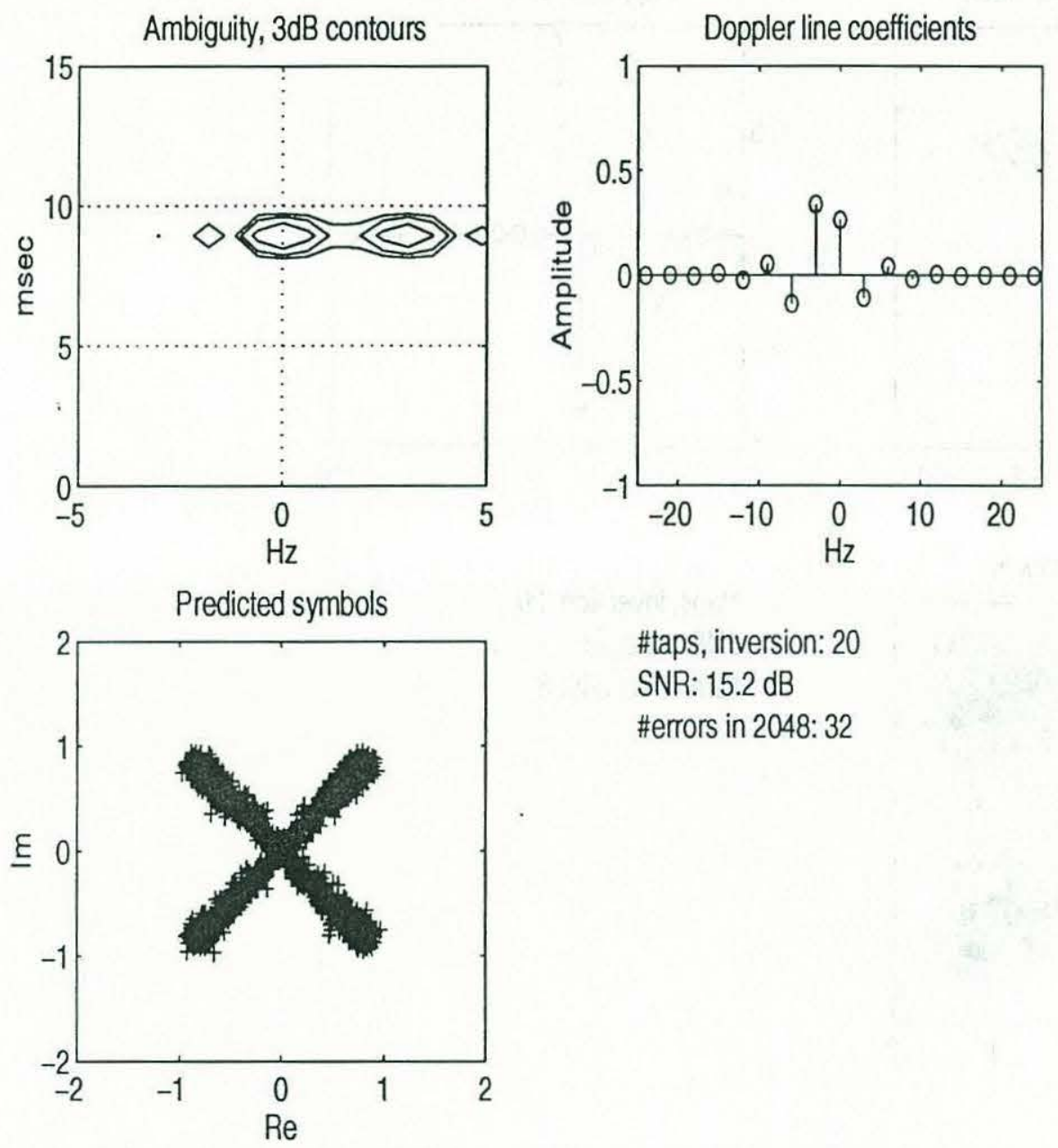

\#taps, inversion: 20

SNR: $15.2 \mathrm{~dB}$

\#errors in 2048: 32

Figure 4-44: Decoding of Doppler spread data with a constrained FFS Doppler line where the constraint is to have unity white noise Doppler line gain. The channel has two frequencies with almost equal magnitude. 

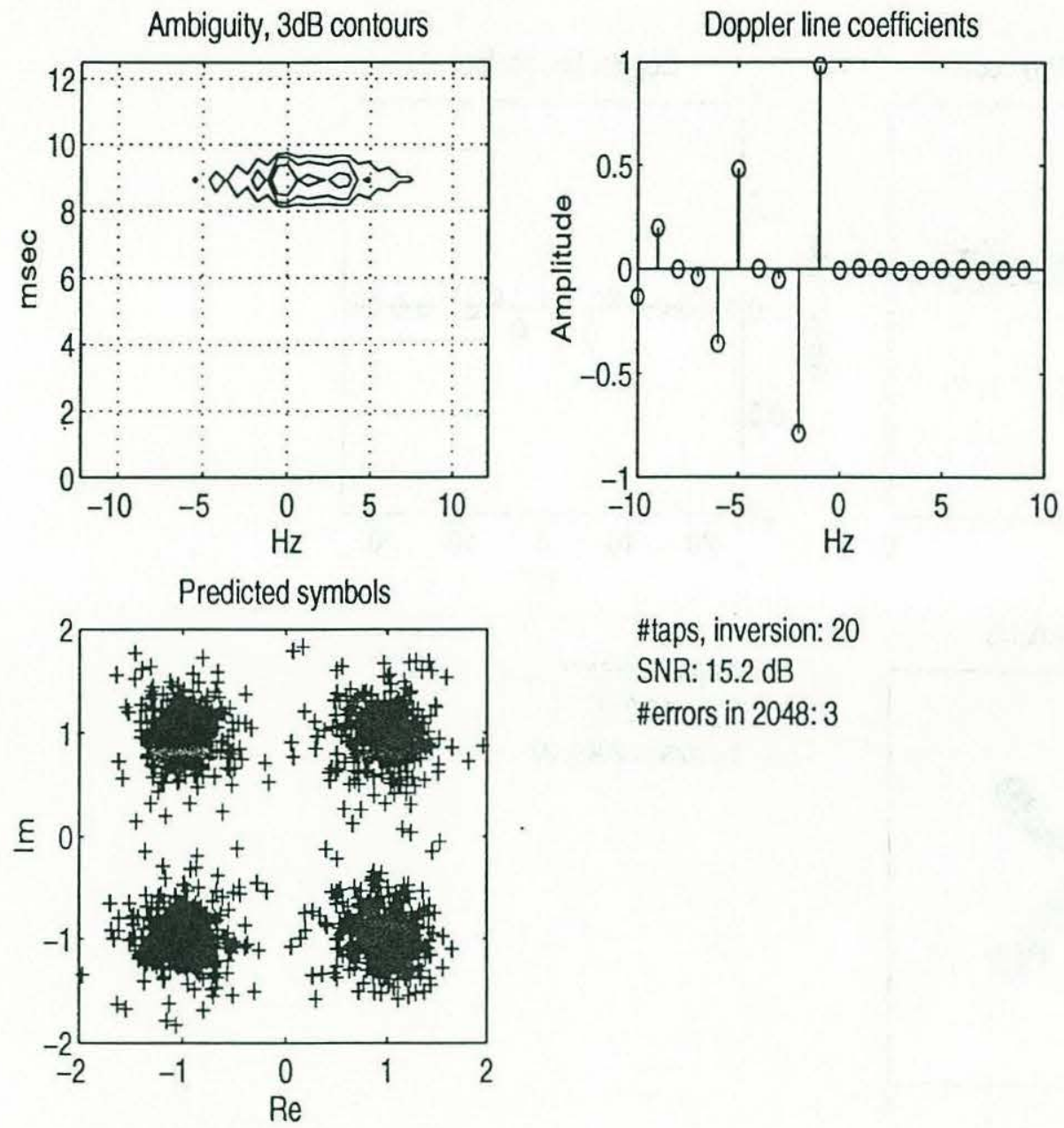

\#taps, inversion: 20

SNR: $15.2 \mathrm{~dB}$

\#errors in 2048: 3

Figure 4-45: Decoding of Doppler spread data with an FFS Doppler line. There are 4 Doppler shifts in the received signal. The channel cross-ambiguity function in upper left panel, the FFS coefficients in upper right panel and the decoded symbol estimates in the lower left panel. 

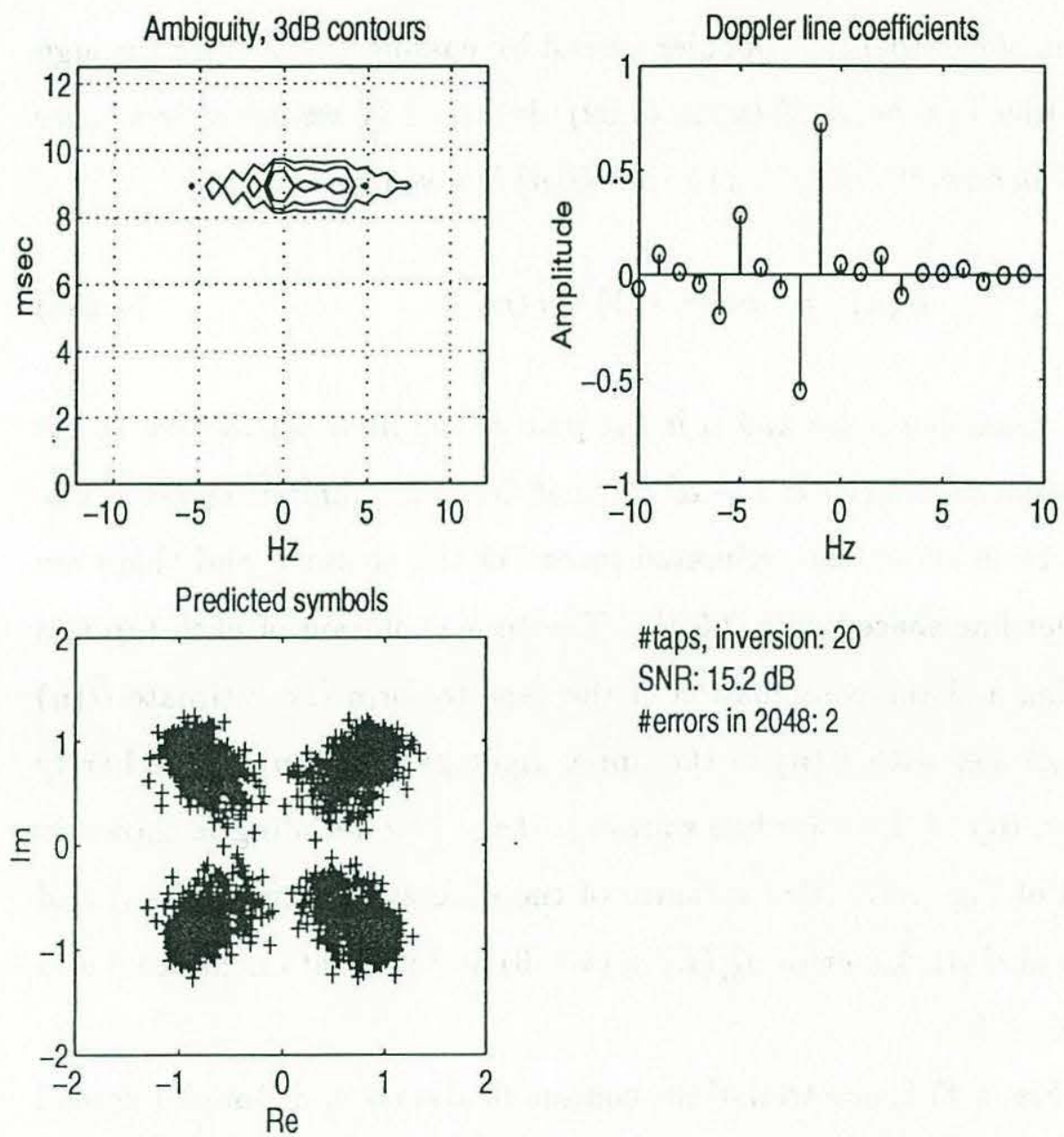

\#taps, inversion: 20

SNR: $15.2 \mathrm{~dB}$

\#errors in 2048: 2

Figure 4-46: Decoding of Doppler spread data with a constrained FFS Doppler line where the constraint is to have unity white noise Doppler line gain. There are 4 Doppler shifts in the received signal. The channel cross-ambiguity function in upper left panel, the FFS coefficients in upper right panel and the decoded symbol estimates in the lower left panel. 


\section{Continuous Doppler spread}

Some ocean processes that give Doppler spread are better modeled by a dense Doppler spectrum rather than a spectrum generated from discrete tones. An example of such a process can be the spreading in frequency of a ray reflected from a rough ocean-surface. We model this Doppler spread by passing white noise through a lowpass filter in order to generate $U(n)$ in (4.99). In Fig. 4-47 we use a first order IIR filter with $3 \mathrm{~dB}$ bandwidth of $10 \mathrm{~Hz}$ so that $U(n)$ is given by

$$
U(n)=\alpha U(n-1)+v(n)
$$

where $v(n)$ is white Gaussian noise and $\alpha$ is the pole of the filter set to give $10 \mathrm{~Hz}$ bandwidth. The variance of $v(n)$ is $1-\alpha^{2}$ so that $U(n)$ has unit variance. The cross-ambiguity function shows the estimated spread of the channel, and there are 5 taps in the Doppler line spaced with $2.5 \mathrm{~Hz}$. The time evolution of each tap is a time-varying function and the combination of the taps to form the estimate $\hat{U}(n)$ of $U(n)$ is shown together with $U(n)$ in the upper right panel. $\hat{U}(n)$ is the barely visible dotted line on top of the solid line showing $U(n)$. The decoding is shown in the lower left panel of Fig. 4-47. The variance of the difference between $U(n)$ and $\hat{U}(n)$ is the total channel tracker error $\sigma_{h_{e}}^{2}(L)$ in (4.150) and its relationship to $\lambda$ and $L$ is shown in Section 4.3.8.

The example in Fig. 4-47 is one trial of the communication over a Doppler spread channel with bandwidth $10 \mathrm{~Hz}$. This is the reason for the observed peaks in the crossambiguity function of Fig. 4-47. By running the receiver many times with the same Doppler spread we compute empirically the probability of decoding error, and the result is shown in Fig. 4-49. This shows receiver performance vs Doppler spread when the delay spread is zero and the SNR is $12 \mathrm{~dB}$.

The result of the channel in Fig. 4-48 with the continuous Doppler spread of (4.258) using the FFS Doppler line of (4.131) shows unsuccessful decoding for the 


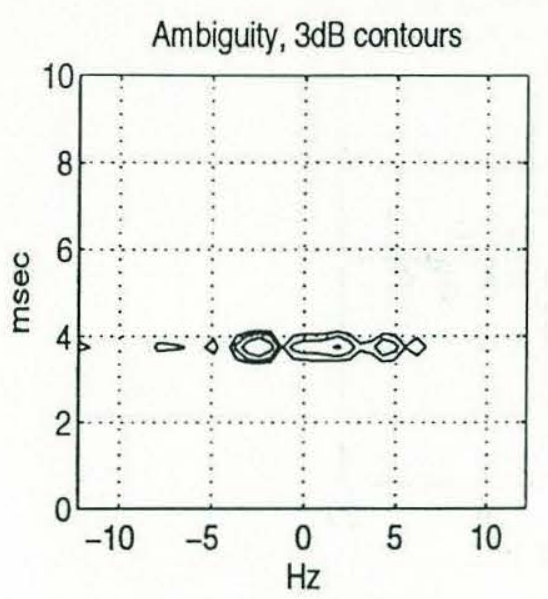

Predicted symbols

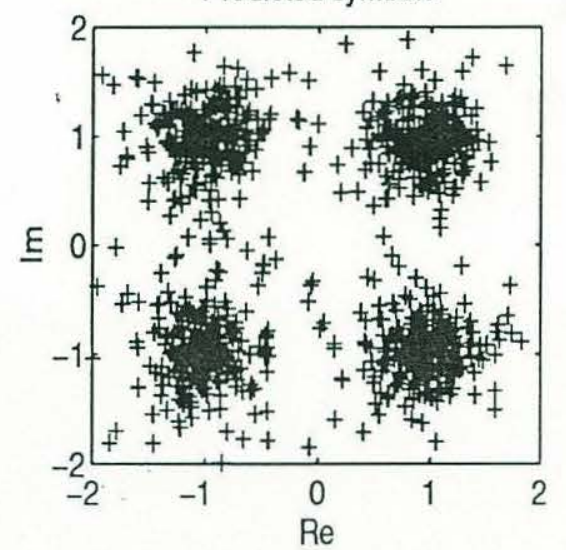

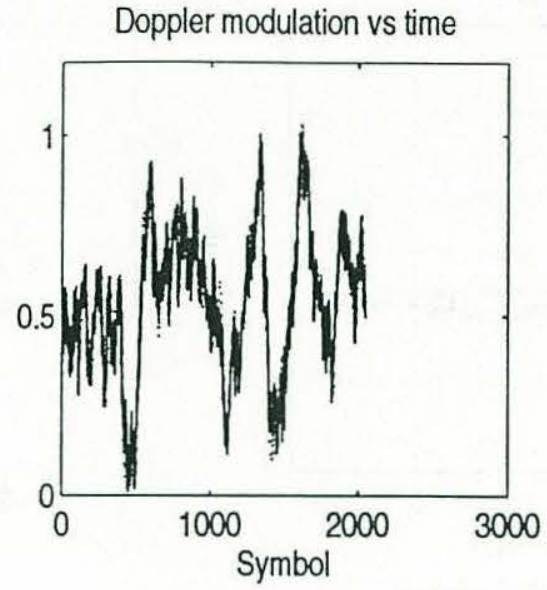

\#taps, tracking: 5

SNR: $15.2 \mathrm{~dB}$

\#errors in 2048:57

lambda: 0.970

Figure 4-47: Decoding using the IFS Doppler line and recursive channel tracker. The Doppler spread is generated from band limited white noise.

following reasons: The Doppler spectrum is nearly flat over its bandwidth, so that there are many taps in the channel response that have nearly equal magnitude. The FFS receiver needs very many taps in order to compensate this channel; we explain this in the discussion of Fig. 4-43.

Summary The receiver in Section 4.3.4 is used to decode data received over timevariant rays with different Doppler shifts arriving at the same time in Fig. 4-40. We compare the IFS and FFS Doppler line based receivers in Fig. 4-41-4-45 which 

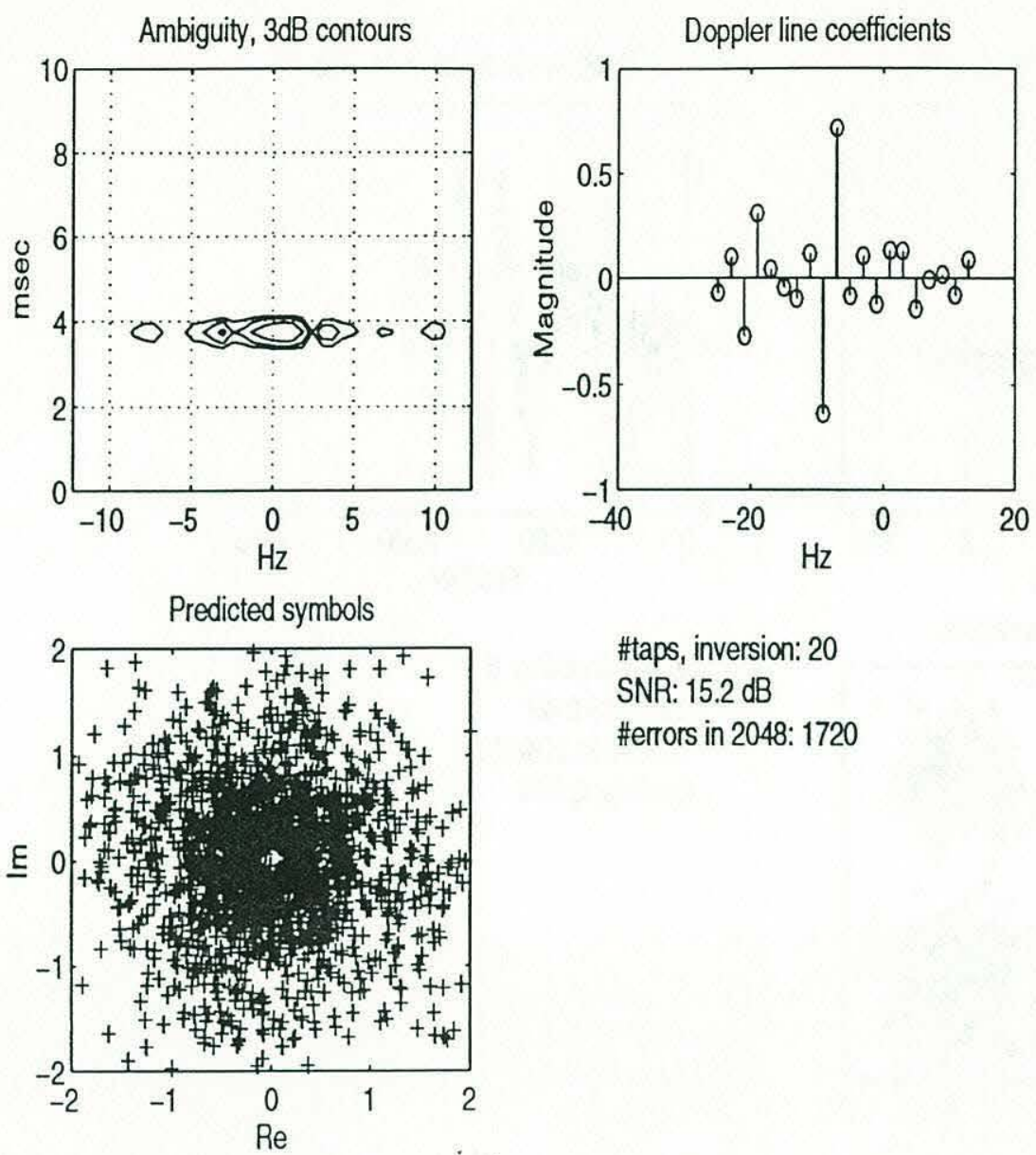

\#taps, inversion: 20

SNR: $15.2 \mathrm{~dB}$

\#errors in 2048: 1720

Figure 4-48: Decoding using the FFS Doppler lines and the block channel estimator. The channel response contains a continuous Doppler spread with nearly flat spectrum over its bandwidth.

demonstrates the noise enhancement sometimes present in the IFS based receiver. A continuously Doppler spread signal is decoded in Fig. 4-47 where the IFS based receiver updated with TU-RLS is used. The characterization of this receiver in terms of probability of decoding error is shown in Fig. 4-49. The FFS based receiver is not useful for this type of Doppler spread and this is shown in Fig. 4-48 and explained in the discussion of Fig. 4-43. 


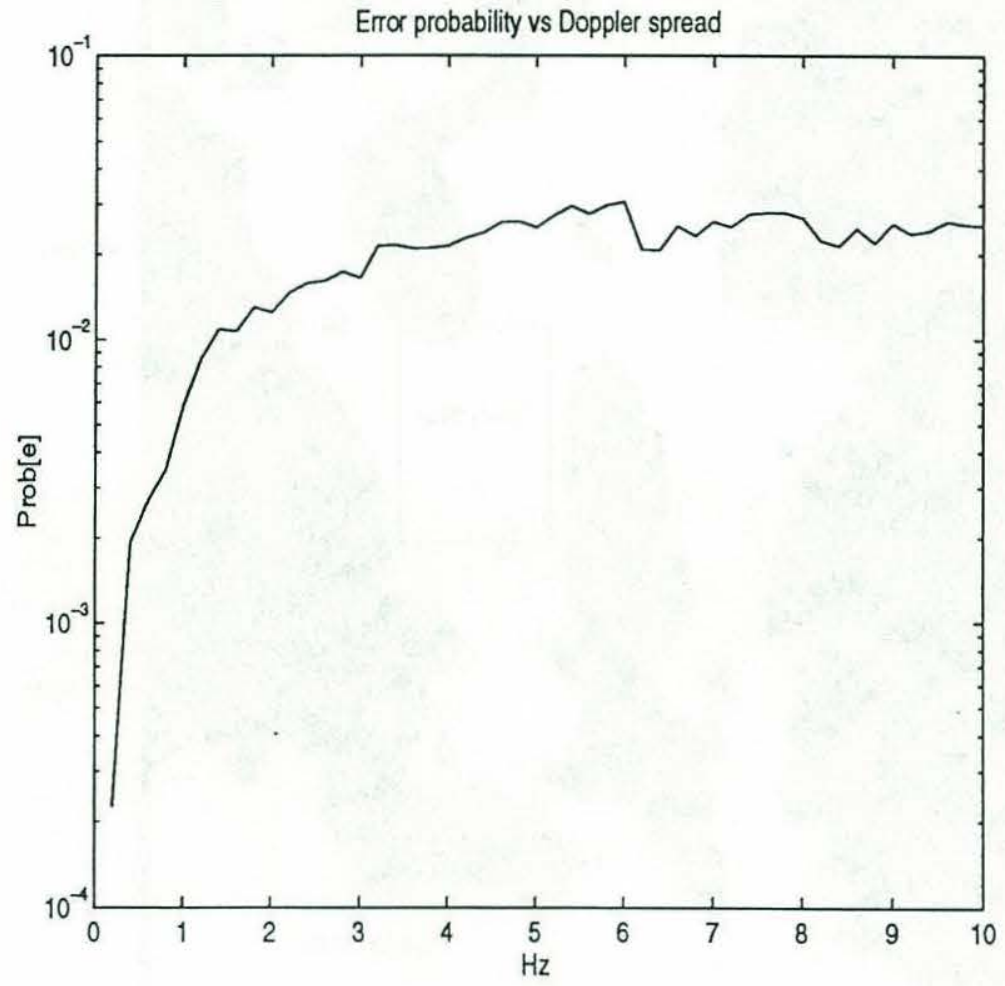

Figure 4-49: Probability of decoding error vs Doppler spread when there is no delay spread and the SNR is $12 \mathrm{~dB}$. 


\subsubsection{Real data}

Some of the data from the Gould Island experiment is decoded with the receiver based on the TU-RLS derived in this work. A map of the experiment site is shown in Fig. 4-50. The water depth on the experiment site is not exceeding $20 \mathrm{~m}$, so

\section{Narraganset Bay}

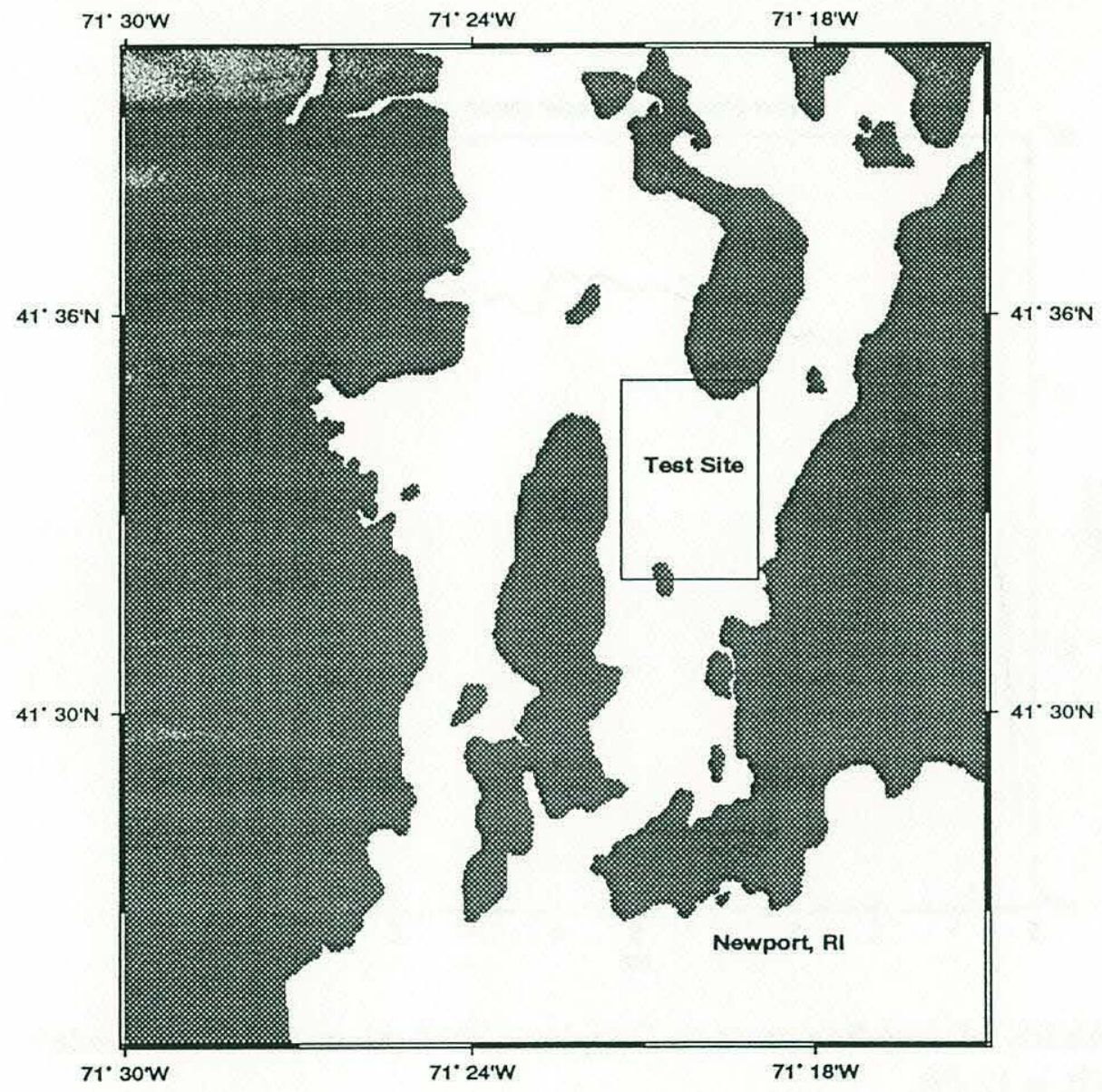

Figure 4-50: Map over the site of the Gould Island experiment. The island intersected by the lower border of the rectangle is Gould Island where the receiver is mounted.

that we have a shallow water scenario with a medium range communication channel for all of the transmissions shown in this section. Refer to Section 3.2 for more information about the experiment. The DFE analyzed in Section 4.2 is also used to decode data from this experiment, and Fig. 4-51 shows estimated symbols for 
both receivers on four data transmissions with bit rates varying in the range $1.2-5$ $\mathrm{kbit} / \mathrm{sec}$. The horizontal distance is $1-2 \mathrm{~km}$, the number of training symbols for the TU-RLS receiver is 100, the total number of decoded symbols is 1024 and the SNR is $10-20 \mathrm{~dB}$. The underwater communication channel for these transmissions is not severely spread in either Doppler or delay, so the result merely demonstrates the ability to decode data acquired from the ocean.
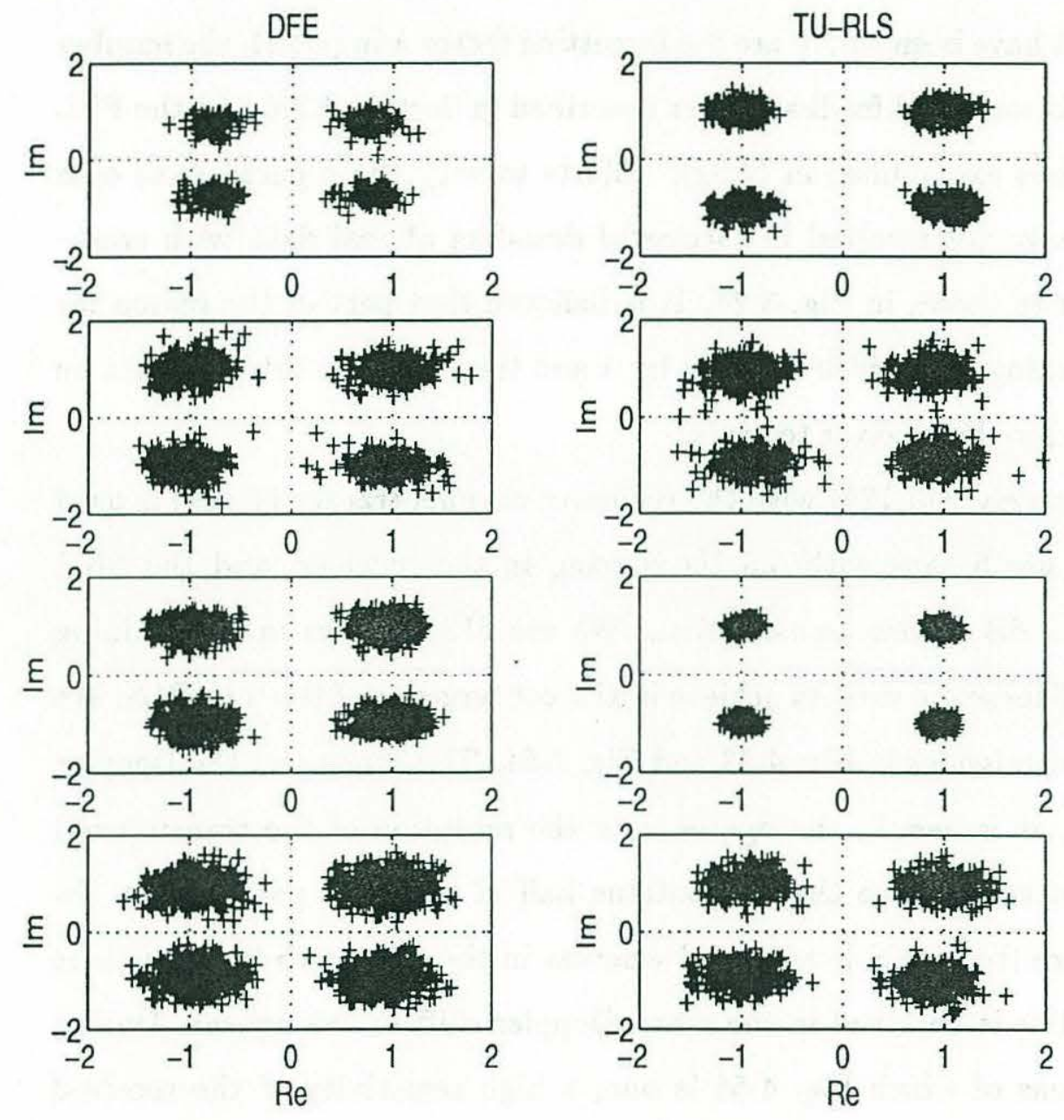

Figure 4-51: Eye pattern from the decoding of some data transmissions in the ocean. The left column shows results obtained with the DFE, and the right column shows results when using the new receiver.

During the same experiment also some purely Doppler spread data were acquired, 
and Fig. 4-52 shows the decoding of one typical transmission. The Doppler spread of approximately $2 \mathrm{~Hz}$ is significant considered the relatively low symbol rate of $600 \mathrm{symb} / \mathrm{sec}$. As described in Section 3.2.4 the transmitter was suspended from a drifting vessel, and the wave height was approximately $30 \mathrm{~cm}$ so the observed Doppler spread is believed to stem from transmitter motion.

The DFE with a PLL as described in Section 4.2 has been used extensively in an effort to decode these data. The user chooses the configuration of this receiver, and the parameters that have been varied are the forgetting factor $\lambda$ in (4.22), the number of taps in the feedforward and feedback filter described in Section 4.2.4 and the PLL loop filter parameters exemplified in (4.82). Efforts to vary these parameters over reasonable spans have not resulted in successful decoding of real data with crossambiguity function as shown in Fig. 3-24. It is believed that part of the reason for this is that the tracking bandwidth as given by $\lambda$ and the PLL loop filter can not be made wide enough for the receiver to track.

The IFS based receiver (4.120) with the recursive channel tracker (4.109) is used in Fig. 4-52. We use 5 taps with $0.5 \mathrm{~Hz}$ spacing in the receiver, and the SNR is approximately $12 \mathrm{~dB}$ in this transmission. We use 512 samples in the training sequence, and 64 of these are used to achieve initial convergence of the TU-RLS. We show two more transmissions in Fig. 4-53 and Fig. 4-54. The range and the Doppler spread is different, as is seen in the figures, and the reception of the transmission in Fig. 4-54 was interrupted so that it contains half of the data packet only. In Fig. 4-53 the transmitter vessel is anchored whereas in the other two transmissions it is drifting, and this is observed in the mean Doppler shift of the figures. During the the transmissions of which Fig. 4-54 is one, a high sensitivity of the received signal level to transmitter depth was observed. A change of the transmitter depth of only 1-2 m produced $10 \mathrm{~dB}$ difference in received signal energy. This indicates that a duct was present, and the available sound speed measurements supports this suggestion. 

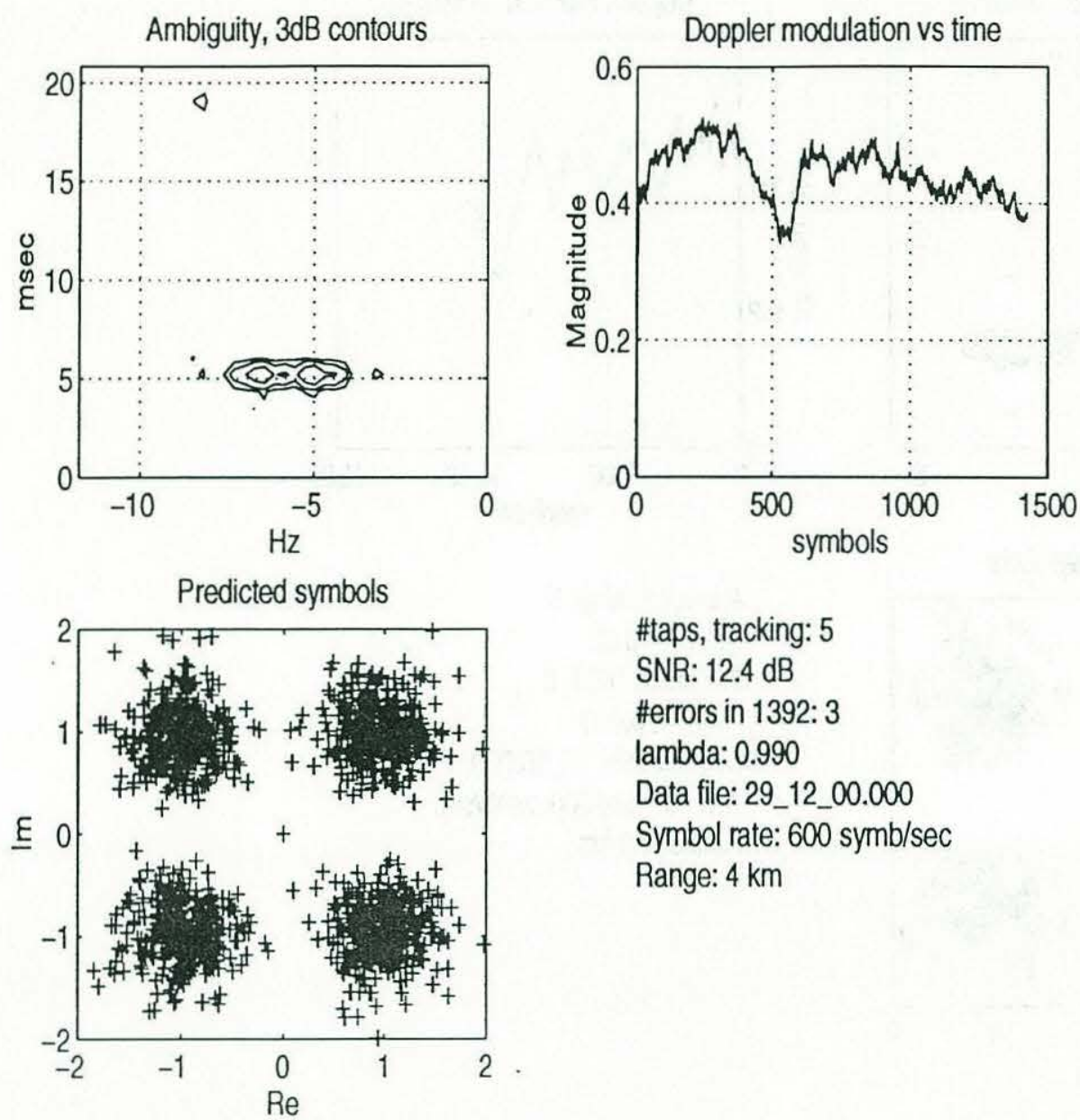
\#taps, tracking: 5
SNR: $12.4 \mathrm{~dB}$
\#errors in 1392: 3
lambda: 0.990
Data file: 29_12_00.000
Symbol rate: $600 \mathrm{symb} / \mathrm{sec}$
Range: $4 \mathrm{~km}$

Figure 4-52: Decoding and channel tracking of purely Doppler spread data from the Gould Island experiment. The mean Doppler is from transmitter vessel drift.

We further illustrate the Doppler spread present in these channels by showing a series of impulse response estimates in Fig. 4-55. The estimates $\hat{h}(n, m)$ are computed by

$$
\hat{h}(n, m)=\sum_{k=n N+1}^{(n+1) N} z^{*}(m+k) y(k) .
$$

We see one dominant signal return in accordance with the cross-ambiguity function in Fig. 4-53, and the fading rate of about $1.5 \mathrm{~Hz}$ in this particular interval of the 

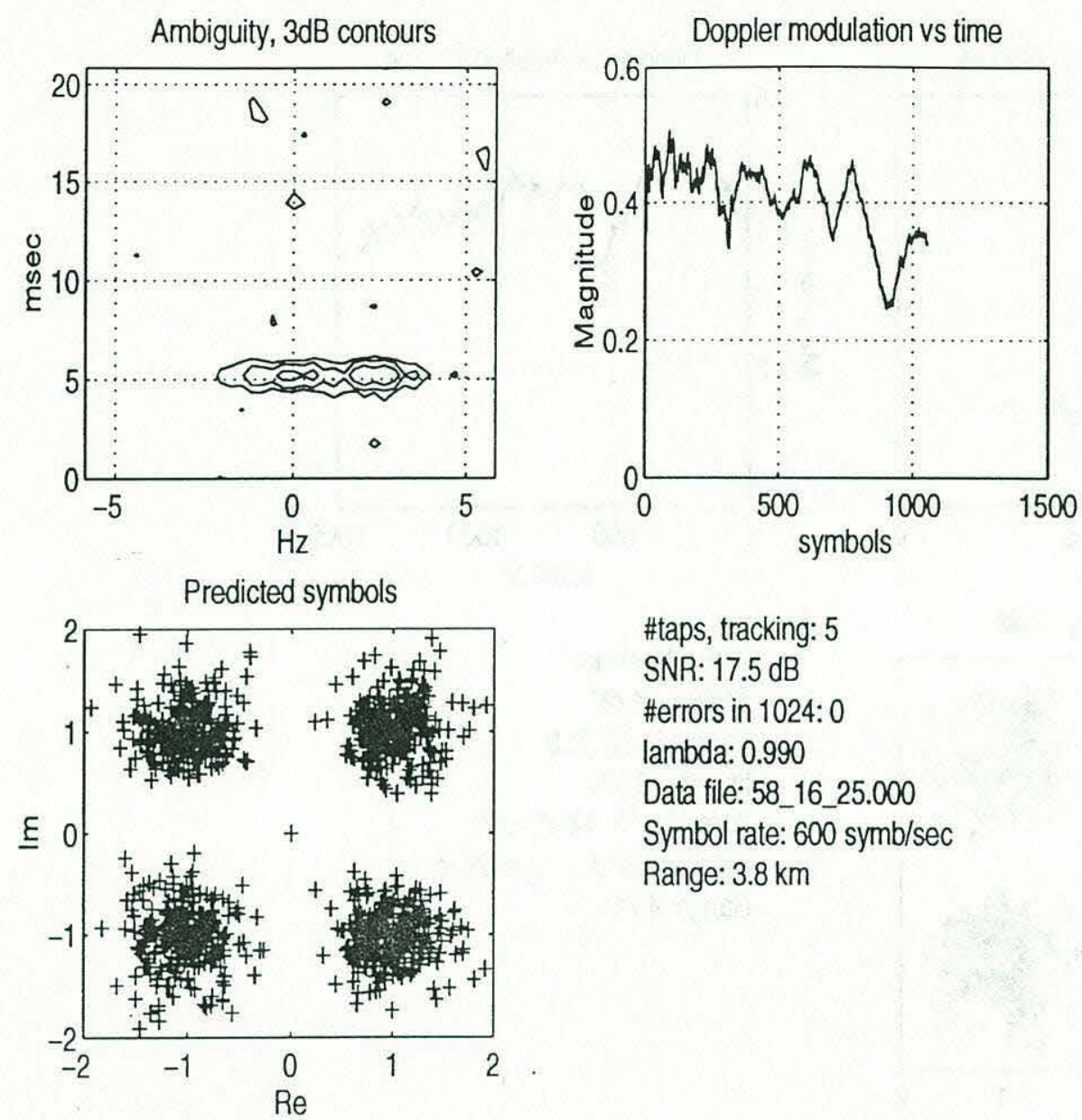

\#taps, tracking: 5

SNR: $17.5 \mathrm{~dB}$

\#errors in 1024: 0

lambda: 0.990

Data file: 58_16_25.000

Symbol rate: $600 \mathrm{symb} / \mathrm{sec}$

Range: $3.8 \mathrm{~km}$

Figure 4-53: The transmitting vessel is anchored, but it has a roll producing Doppler spread.

data transmission. In Fig. 4-56 we show the time evolution of the largest tap value from Fig. 4-55. The marks on this graph are equidistant in time, and the interval between adjacent marks is $0.1 \mathrm{sec}$. By examining Fig. 4-56 we find parts of the trajectory where the phase change is $\pi / 4$ between two marks corresponding to an instantaneous Doppler frequency of $2.5 \mathrm{~Hz}$. This is in good correspondence with the cross-ambiguity function of Fig. 4-53 

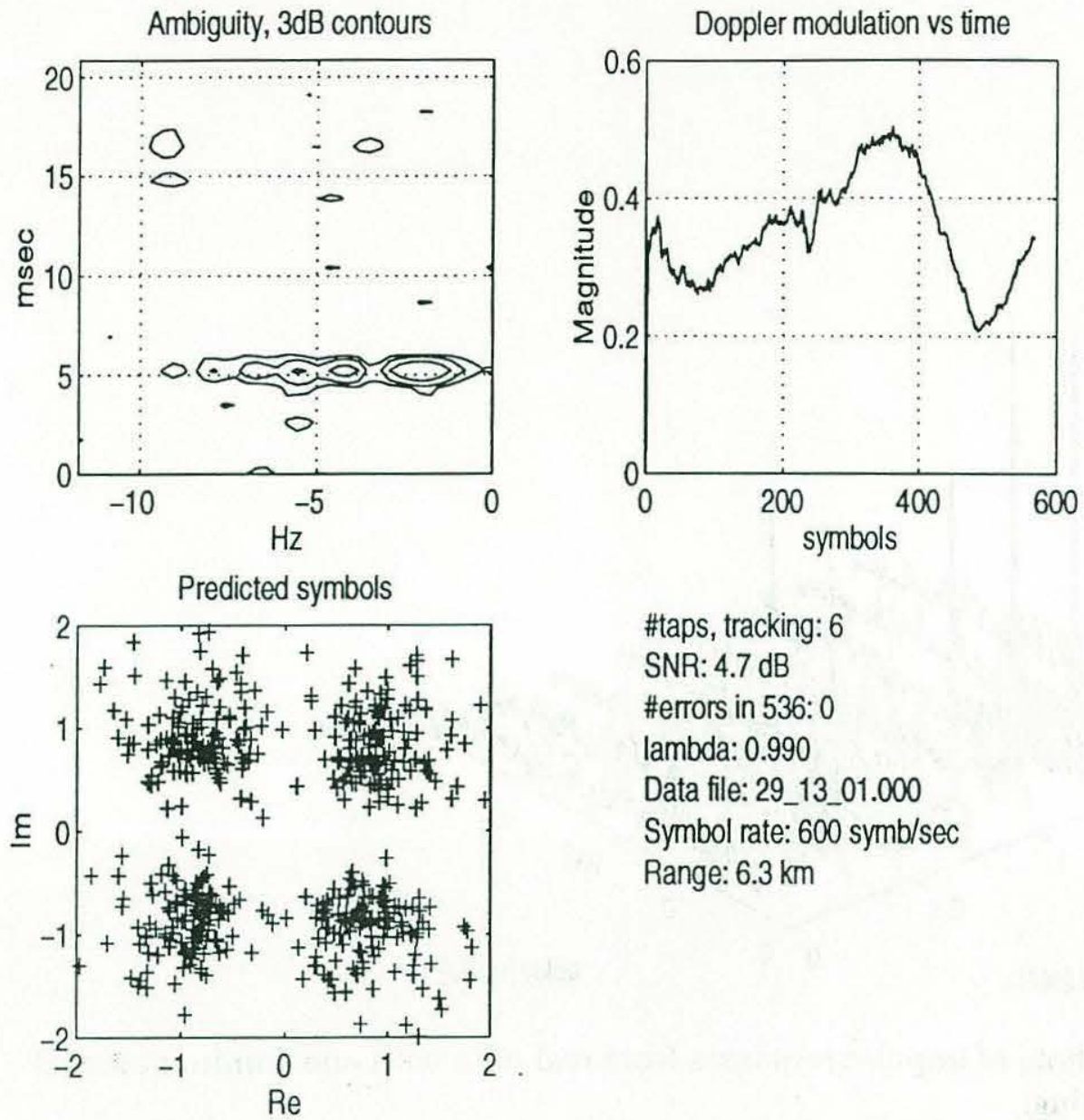

\#taps, tracking: 6

SNR: $4.7 \mathrm{~dB}$

\#errors in 536: 0

lambda: 0.990

Data file:29_13_01.000

Symbol rate: $600 \mathrm{symb} / \mathrm{sec}$

Range: $6.3 \mathrm{~km}$

Figure 4-54: The transmitting vessel was drifting, and the received signal level is very sensitive to transmitter depth.

Summary A brief comparison of a DFE and the TU-RLS receiver is shown in Fig. 4-51, and this verifies equal performance on data not severely spread in Doppler. The data in Fig. 4-52-4-54 have only been decoded with the TU-RLS receiver, and this verifies the capability of this receiver on purely Doppler spread data. The reason for being unable to decode these data with other receivers is believed to be the observed Doppler spread. We present impulse response estimates in Fig. 4-55, and by looking closer at the time evolution of these estimates in Fig. 4-56 we find instantaneous Doppler frequencies of the same size as shown by the cross-ambiguity 
Impulse response estimates

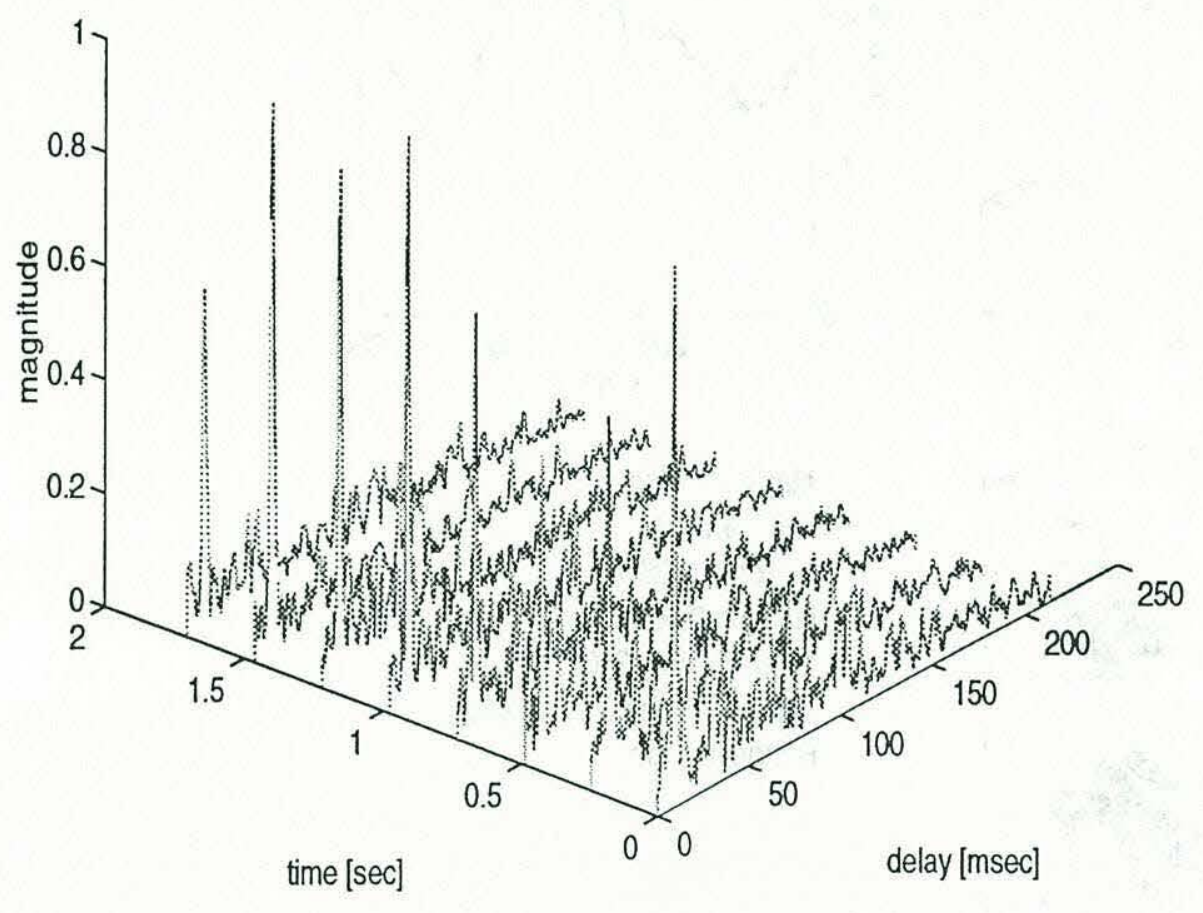

Figure 4-55: Snapshots of impulse responses from real data with one dominant signal return which is fading.

function in Fig. 4-53. 


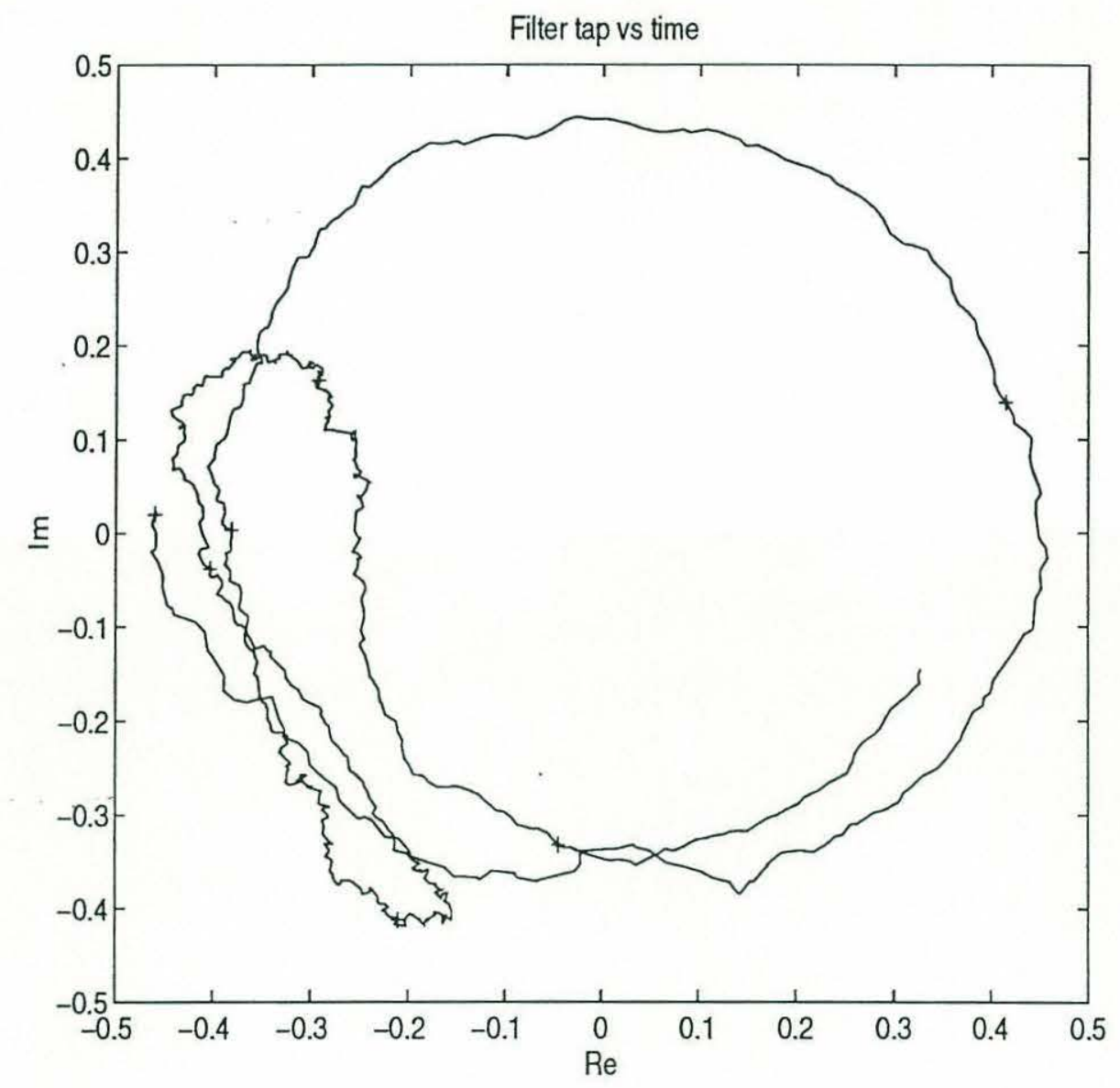

Figure 4-56: Time evolution of a Doppler spread signal return from the Gould Island experiment. 


\section{Chapter 5}

\section{Conclusion}

In this chapter we give an overview of the work in this thesis in the framework of previously reported results, and we also suggest some future directions. The overview is contained in Section 5.1, and it naturally leads to Section 5.2 where we point out some possible future tasks not covered in this thesis.

\subsection{Summary of thesis}

\subsubsection{Background}

The amount of work reported on underwater acoustic communication is huge both when it comes to simulation studies and implemented systems. In Chapter 1 we give an overview of representative publications without attempting to be exhaustive in referencing the literature. The main purpose of this part is to illustrate that underwater communication channels are very diverse, and this is reflected in the fact that no predominant modulation scheme or receiver structure has emerged as a standard. On this background the thesis is very specific since we treat only QPSK modulation which constrains the scope of the work to be coherent communication. We comment further on this in Section 5.2.4. 


\subsubsection{Theory}

The most important assumption pertaining to the theoretical treatment of underwater communication channels is the WSSUS assumption. The channel scattering is modeled by random processes and the WSSUS assumption is that each scattering process is wide sense stationary (2.4), and the scattering processes from two processes are uncorrelated (2.6). This enables us to define the channel scattering function (2.7) as a two dimensional power spectral density in delay and Doppler.

The well known ambiguity function is developed into the cross-ambiguity function (2.32), and it is used to estimate the channel scattering function from the input and output data to the channel. We emphasize on Doppler spread, and we point out that the shape of the cross-ambiguity function can not uniquely tell us what physical phenomenon that caused the Doppler spread. We use the narrowband cross-ambiguity function, and we show that the narrowband assumption is not always satisfied in the underwater communication channel.

There is no fundamentally new theory in this thesis, but the combination of some pieces from the literature serves to develop the Doppler line which is a concept that is not commonly known. A Doppler line is the frequency domain counterpart of a delay line which is better known as a filter. We show that these devices are useful for compensating Doppler spread channels.

\subsubsection{Channel modeling}

The delay and Doppler spreads observed in the channel characterization from real data presented in Chapter 3 is linked to ocean physical processes by means of a simulator. It is based on a ray representation of the acoustic field and a time-variant FIR filter (3.3). The input to the simulator are various parameters controlling the ray propagation, such as sound speed profile, surface roughness, etc, and the output is the signal at the receiver. Thus we achieve a controlled experiment where we know which 
factors that contribute to the channel structure. This is used to motivate physical explanations for the delay and Doppler spread observed from real data in Fig. 3-143-24. We also illustrate the wide diversity of underwater communication channels in Fig. 3-7-3-11, and this motivates the self imposed restriction in this thesis to only consider the subclass of underwater communication channels that have significant Doppler spread.

\subsubsection{Receiver derivation and analysis}

We present the ML receiver for doubly spread channels in the case of known channel response (4.4). This is found to be very complex in a representative set of underwater communication channels, and we point out that the complexity increases further in the realistic case of unknown channel response. This motivates receivers derived from the suboptimum criterion of MMSE, and we first consider the DFE in Fig. 4-4 which is a common receiver both in underwater communication channels and other communication channels. We carry out performance analysis in the special case of a purely Doppler spread signal in Section 4.2, and it is found that the adaptive DFE is not capable of compensating realistically Doppler spread channels (4.41). Moreover, we point out in Section 4.2.6 unexpected and unwanted system behavior in the composite receiver containing the DFE and a PLL in Fig. 4-13, and we show this system behavior on real data in Fig. 4-19. This motivates the new receiver that is based on the TU-RLS algorithm (4.92) and (4.93). The structure of this receiver is based on the findings that 1) different rays may have different Doppler shifts and 2) the main limitation of a DFE operating in Doppler spread is the feedback of past symbols for the decoding. Thus the adaptive algorithm called TU-RLS is used in the channel tracking, allowing 1), and a FIR filter (4.138) is used for decoding, eliminating the limitation 2).

The Doppler spread under which the basic receiver (4.92) and (4.93) can operate is given by the exponential weighting $\lambda$, and this receiver is developed further to ac- 
commodate larger Doppler spreads. The Doppler lines introduced in Section 2.4 are used to achieve communication over purely Doppler spread channels, and several extended receivers (4.107), (4.109), (4.120) and (4.126) based on various combinations of FFS Doppler lines, IFS Doppler lines, block processing and recursive processing are suggested.

We derive a procedure for initializing this receiver (4.189) with the right number of taps and proper delays and Doppler shifts by means of the cross-ambiguity function. We suggest the update of these parameters to be carried out periodically by a constrained search over the updated cross-ambiguity function in the same manner as for the tap initialization.

We characterize the receiver performance in presence of noise, delay spread and Doppler spread by computing the probability of decoding error (4.232), and we also show that the receiver stability is determined by the conditioning of the matrix that is inverted and recursively updated by the TU-RLS. The structure of this matrix is governed by the delay and Doppler spread of the channel. We show in the case of purely Doppler spread channels that the frequency tap spacing limits the receiver stability Fig. 4-33, and in the case of purely delay spread channels the receiver stability is given by (4.229) and (4.230).

The various receivers are verified on simulated Doppler spread data, and we demonstrate the ability to track the channel and decode data both in the presence of Doppler spread containing discrete frequencies in Fig. 4-36 and continuous bands in Fig. 4-47. The decoding of real data is shown in Fig. 4-51 but these data are not severely Doppler spread, and thus the example merely shows successful decoding in a scenario where both the DFE and the TU-RLS receiver works. Another series of real data is shown in Fig. 4-52-4-54, and these data are severely Doppler spread. The cross-ambiguity function from these data show a single signal return with Doppler spread in the range $2-5 \mathrm{~Hz}$, and this is a significant frequency dispersion. We show, by using the IFS Doppler line and the TU-RLS to update its coefficients, successful 
decoding of these data. The Doppler spread in these data is believed to stem from transmitter motion since the transmitter is suspended from a surface vessel in sea state 1 . This is a very common scenario in underwater acoustic communication, and we demonstrate a significant advance by being able to communicate in the presence of this Doppler spread.

\subsection{Future directions}

In the course of the work there were numerous issues encountered that have not been addressed or only briefly treated. We now comment on the most obvious ones, and the purpose is to show potentially useful ways for continuing this work.

\subsubsection{DFE adapted with TU-RLS}

The DFE is extensively analyzed in the literature, see [82] and references therein, and it has both shortcomings and advantages compared to the FIR decoder used in Fig. 4-20. One important advantage is the ability to work well over delay spread channels with returns of almost equal magnitudes. This is a shortcoming of the FIR decoder in Fig. 4-20 as demonstrated by Fig. 4-37. There is nothing principally preventing the TU-RLS algorithm from being used with a DFE. We note that a potential problem with this approach is the error propagation of the DFE which is much more dramatic than the error propagation of the TU-RLS receiver described

in Section 4.3.8. Also the DFE is a non-linear device making analysis and quality assessments more difficult. Nevertheless, a DFE adapted with TU-RLS has the potential to outperform the receiver in this thesis under specific circumstances. 


\subsubsection{Error coding}

The Doppler spread of a channel is observed in the time domain as fading i.e., in a time interval with a channel fade the signal is severely attenuated. One common way of combating this signal loss is by means of coding. The price paid in terms if lower bit rate because of code redundancy is sometimes small compared to the increased reliability of the communication system. Coding in underwater communication channels is reported in [19], and based on the findings here and in this thesis we suggest that coding is a way of making communication over Doppler spread channels more reliable that should be utilized better in underwater communication channels.

\subsubsection{Simulation}

The simulation of the channel response as discussed in Chapter 3 is essential in order to understand the physical ocean processes that contribute to Doppler spread and the relative importance among these processes. We find that relative platform motion and ocean-surface motion give significant contributions to Doppler spread in the frequency range $5-50 \mathrm{kHz}$. To this end several improvements is suggested both to predict Doppler spread from the above mentioned sources and also incorporate new sources of Doppler spread.

The model for ocean-surface Doppler spread is based on the Pierson-Moskowitz surface wave spectrum and the formulation in [14]. This approach uses the method of tangent planes to calculate the reflected field, and this approximation may be inaccurate. Also the Eckart formulation for the reflection coefficient could be replaced with more accurate albeit complex computation. A potentially useful starting point for replacing the existing ocean-surface formulation is the work by Dowling and Jackson [27].

There are many other known sources of time variance in the ocean, and these also produce Doppler spread. In particular the effect of turbulence and internal 
waves on the Doppler spread is not addressed in this thesis. In order to incorporate such effects the current ray formulation must be replaced with a range dependent representation.

\subsubsection{Modulation}

The scope of this thesis is very limited when it comes to modulation, and part of the reason for this is that the available database of ocean acquired data mostly is QPSK. This is however not a reason to omit other modulation schemes, and a particularly interesting modulation to consider in Doppler spread channels is the multi tone modulation; see [9] and references therein. This gives flexibility in the tradeoff between the frequency duration (bandwidth) and time duration of a symbol that is advantageous in Doppler spread channels. Also many of the regular modulation schemes such as FSK could be revised in order to quantify their robustness compared to QPSK on Doppler spread channels.

\subsubsection{Combined spatial and temporal processing}

The approach to communication used herein assumes that only one sensor is available at the receiver, or that if a number of sensors are available the optimal way of combining them is not a function of sensor location. E.g., the DFE in Fig. 4-17 adds the signals from all the feedforward sections. The impulse response of underwater communication channels usually contains several rays arriving at the receiver from different directions, and it is the relative delay and Doppler between all the rays that give the total spread of the channel. Thus a very straightforward way of decreasing both delay and Doppler spread is to use a spatial filter in the receiver to attenuate rays from specified directions and thereby simplifying the impulse response. This shifts the processing from temporal to spatial, and there is nothing that suggests the optimal receiver to only use temporal and no spatial processing. Thus a receiver 
that combines spatial processing, known as beamforming, and temporal tracking of the impulse response is a good candidate to improve our receiver.

\subsubsection{Channel representation}

The WSSUS assumption that we present in Chapter 2 is at the heart of our channel model, and it has wide implications for the design of the receivers in Chapter 4. The assumption is very common, and very little work on LTV systems has been encountered where this assumption is not adopted. There are situations in underwater communication channels where the assumption is broken. In general, a process is very unlikely to be WSS if the time interval over which the process is observed gets large. It can be argued that the US assumption in the extreme of very closely spaced scatterers is not true. This is treated in a paper by Bello [7], and the concept of quasi WSSUS (QWSSUS), where the channel "behaves" like a WSSUS, is introduced. Useful knowledge could be obtained by investigating the borders of validity of the QWSSUS assumption in underwater communication channels, and propose a model for non-QWSSUS channels. 


\section{Appendix A}

\section{Stability of the stationary region}

The derivative needed to find the system matrix $\mathrm{A}$ is not uniquely defined around the stationary region $\mathrm{x}_{*}$, and we have

$$
A=\frac{\partial \mathrm{f}}{\partial \mathrm{x}}\left(\mathrm{x}_{*}\right)
$$

The system in equilibrium is shown in fig. A-1. We want to find the evolution of $a$

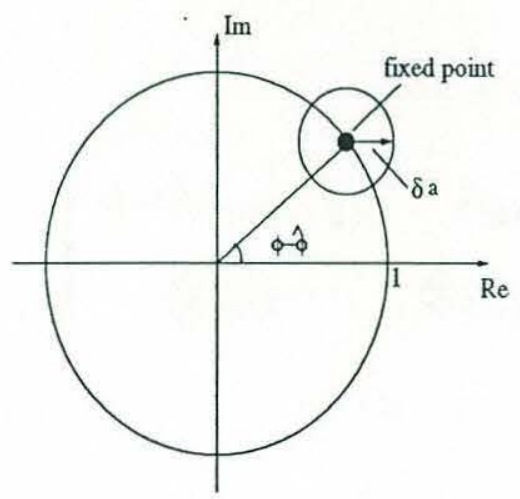

Figure A-1: The filter tap and the PLL phase estimate is in equilibrium when the filter $\operatorname{tap} a$ is on the unit circle.

after a small perturbation in arbitrary direction, and for this purpose we use

$$
\delta a=r e^{j \psi} .
$$


Taking the derivative with respect to $r$, leaving $\psi$ as a parameter, gives us $\mathrm{A}$ as function of the perturbation direction. Since the derivative is not uniquely defined the system matrix is a function of $\psi$.

\section{A.1 Angle PD}

In the case that the difference equation is given by (4.67) we have that

$$
\begin{aligned}
\delta a & =r e^{j \psi} \\
\mathbf{x} & =\left[\begin{array}{l}
r \\
\hat{\phi}
\end{array}\right] \\
\mathbf{f}(\mathbf{x}, \phi) & =\left[\begin{array}{l}
\lambda r e^{j \psi}+(1-\lambda) e^{-j(\phi-\hat{\phi})} \\
\hat{\phi}_{n}-\tan ^{-1} \frac{\sin (\phi-\hat{\phi}+\psi)}{\cos (\phi-\hat{\phi}+\psi)-r}
\end{array}\right] \\
A & =\frac{\partial \mathbf{f}}{\partial \mathbf{x}}\left(\mathbf{x}_{*}\right)=\left[\begin{array}{ll}
\frac{\partial f_{1}}{\partial \mathbf{x}} & \frac{\partial f_{2}}{\partial \mathbf{x}}
\end{array}\right]^{T}
\end{aligned}
$$

and carrying out the differentiation yields

$$
A=\left[\begin{array}{cc}
\lambda e^{j \psi} & j(1-\lambda) e^{-j(\phi-\hat{\phi})} \\
-\frac{1}{1+y^{2}} \frac{\partial y}{\partial r} & -\frac{1}{1+y^{2}} \frac{\partial y}{\partial \hat{\phi}}
\end{array}\right]
$$

where

$$
\begin{aligned}
f_{1}(\mathrm{x}) & =\lambda r e^{j \psi}+(1-\lambda) e^{-j\left(\phi_{n}-\hat{\phi}_{n}\right)} \\
f_{2}(\mathrm{x}) & =\hat{\phi}-\tan ^{-1} y(r, \hat{\phi}) \\
y & =\frac{\sin (\phi-\hat{\phi}+\psi)}{\cos (\phi-\hat{\phi}+\psi)-r}
\end{aligned}
$$




$$
\begin{aligned}
\frac{\partial y}{\partial r} & =\frac{\sin (\phi-\hat{\phi}+\psi)}{(\cos (\phi-\hat{\phi}+\psi)-r)^{2}} \\
\frac{\partial y}{\partial \hat{\phi}} & =\frac{\cos (\phi-\hat{\phi}+\psi)+\sin (\phi-\hat{\phi}+\psi)}{\cos ^{2}(\phi-\hat{\phi}+\psi)}
\end{aligned}
$$

Inserting $r=0$, corresponding to $\mathrm{A}$ in the stationary region, we write the system matrix as

$$
A=\left[\begin{array}{cc}
\lambda e^{j \psi} & j(1-\lambda) e^{-j(\phi-\hat{\phi})} \\
-\frac{1}{1+\frac{\sin (\kappa)^{2}}{\cos (\kappa)} \frac{\sin (\kappa)}{\cos ^{2}(\kappa)}} & -\frac{1}{1+\frac{\sin (\kappa)^{2}}{\cos (\kappa)}} \frac{\cos (\kappa)+\sin (\kappa)}{\cos ^{2}(\kappa)}
\end{array}\right]
$$

where $\kappa=\phi-\hat{\phi}-\psi$. We see that there are specific directions where $[A]_{21}$ and $[A]_{22}$ grow without bound. That corresponds to an unstable system, and thus the stationary region for the angle PD is unstable.

\section{A.2 Imaginary part PD}

Now the difference equation is given by (4.69), and defining the perturbation as in fig. A-1 and (A.2) we have

$$
\mathbf{f}(\mathbf{x}, \phi)=\left[\begin{array}{c}
\lambda r e^{j \psi}+(1-\lambda) e^{-j(\phi-\hat{\phi})} \\
\hat{\phi}-r \sin (\phi-\hat{\phi}+\psi)
\end{array}\right]
$$

We get

$$
A=\left[\begin{array}{cc}
j \lambda e^{j \psi} & j(1-\lambda) e^{-j(\phi-\hat{\phi})} \\
-\sin (\phi-\hat{\phi}+\psi) & 1
\end{array}\right]
$$


For the specific direction $\psi=\hat{\phi}-\phi$ we have that

$$
A=\left[\begin{array}{cc}
j \lambda e^{j \psi} & j(1-\lambda) e^{-j(\phi-\hat{\phi})} \\
0 & 1
\end{array}\right]
$$

and in this case the largest eigenvalue is 1 . There exists a direction for the perturbation such that the system does not return to equilibrium, so in this case it is marginally stable. It is important to note that this analysis is local around the stationary region. We have a nonlinear system, so the conclusions here will not give exhaustive information about the system dynamics. 


\section{Appendix B}

\section{Channel estimation error}

\section{covariance}

In this appendix we motivate the choice of gain in (4.217) and detail the steps in going from (4.216) to (4.221). We first motivate the use of $\tilde{P}(n)$ in (4.217). The TU-RLS minimizes

$$
J=\sum_{m=0}^{n} \lambda^{n-m}\left|y(m)-\mathbf{c}_{0}^{H}(m) A^{m-n} \hat{\mathbf{h}}(n)\right|^{2}
$$

and to find the optimum $\hat{\mathrm{h}}(n)$ we perform $\partial J / \partial \hat{\mathrm{h}}=0$ which yields

$$
\begin{aligned}
\hat{\mathbf{h}}(n) & =\left[\sum_{m=0}^{n} \lambda^{n-m}\left(A^{H}\right)^{m-n} \mathbf{c}_{0}(m) \mathbf{c}_{0}^{H}(m) A^{m-n}\right]^{-1} \sum_{m=0}^{n} \lambda^{n-m}\left(A^{H}\right)^{m-n} \mathbf{c}_{0}(m) y(m) \\
& =R_{c}^{-1}(n) \rho(n) .
\end{aligned}
$$

We see from (B.2) that

$$
\begin{aligned}
R_{c}(n) & =\lambda A^{-H}\left\{\sum_{m=0}^{n-1} \lambda^{n-1-m}\left(A^{H}\right)^{m-(n-1)} \mathbf{c}_{0}(m) \mathbf{c}_{0}^{H}(m) A^{m-(n-1)}\right\} A^{-1}+\mathbf{c}_{0}(n) \mathbf{c}_{0}^{H}(n) \\
& =\lambda A^{-H} R_{c}(n-1) A^{-1}+\mathbf{c}_{0}(n) \mathbf{c}_{0}^{H}(n)
\end{aligned}
$$




$$
\begin{aligned}
\rho(n) & =\lambda A^{-H} \sum_{m=0}^{n-1} \lambda^{n-1-m}\left(A^{H}\right)^{m-(n-1)} \mathbf{c}_{0}(m) y(m)+\mathbf{c}_{0}(n) y(n) \\
& =\lambda A^{-H} \rho(n-1)+\mathbf{c}_{0}(n) y(n) .
\end{aligned}
$$

The following equation is a slight generalization of the derivation in [64], p.306 and it follows from (B.2)-(B.4):

$$
\begin{aligned}
\hat{\mathbf{h}}(n) & =R_{c}^{-1}(n)\left[\lambda A^{-H} \rho(n-1)+\mathbf{c}_{0}(n) y(n)\right] \\
& =R_{c}^{-1}(n)\left[\lambda A^{-H} R_{c}(n-1) \hat{\mathbf{h}}(n-1)+\mathbf{c}_{0}(n) y(n)\right] \\
& =R_{c}^{-1}(n)\left[\left\{R_{c}(n)-\mathbf{c}_{0}(n) \mathbf{c}_{0}^{H}(n)\right\} A \hat{\mathbf{h}}(n-1)+\mathbf{c}_{0}(n) y(n)\right] \\
& =A \hat{\mathbf{h}}(n-1)+R_{c}^{-1}(n) \mathbf{c}_{0}(n)\left[y(n)-\mathbf{c}_{0}^{H}(n) A \hat{\mathbf{h}}(n-1)\right]
\end{aligned}
$$

where we have used (B.4) to get the first expression, (B.2) to get the second expression and (B.3) to get the third expression. We recognize the last expression as the update step of the TU-RLS as given in (4.92). Thus we identify the gain vector as

$$
\mathrm{k}(n)=R_{c}^{-1}(n) \mathrm{c}_{0}(n)
$$

We write

$$
\begin{aligned}
R_{c}(n) & =\frac{1}{1-\lambda} \sum_{m=0}^{n}(1-\lambda) \lambda^{n-m}\left(A^{H}\right)^{m-n} \mathbf{c}_{0}(m) \mathbf{c}_{0}^{H}(m) A^{m-n} \\
& =[\chi \tilde{P}(n)]^{-1}
\end{aligned}
$$

where the convenience of the scaling in the second expression is shown below. We get (4.217) by combining (B.6) and (B.7).

In the case of one Doppler coefficient for each delay (B.7) yields

$$
R_{c}(n)=\sum_{m=0}^{n} \lambda^{n-m}\left(A^{H}\right)^{m-n} \mathbf{c}_{0}(m) \mathbf{c}_{0}^{H}(m) A^{m-n}
$$




$$
\begin{aligned}
& =\mathcal{E}\left[\begin{array}{ccc}
\sum_{m=0}^{n}\left(\lambda / \alpha_{0}^{2}\right)^{m-n}\left|z\left(n-l_{0}\right)\right|^{2} & \cdots & \sum_{m=0}^{n}\left(\lambda / \alpha_{0} \alpha_{L-1}\right)^{m-n} z\left(n-l_{0}\right) \times \\
\vdots & z^{*}\left(n-l_{L-1}\right) e^{j 2 \pi(L-1) \Delta \nu(m-n)} \\
\sum_{m=0}^{n}\left(\lambda / \alpha_{0} \alpha_{L-1}\right)^{m-n} z^{*}\left(n-l_{0}\right) \times & \cdots & \sum_{m=0}^{n}\left(\lambda / \alpha_{L-1}^{2}\right)^{m-n}\left|z\left(n-l_{L-1}\right)\right|^{2} \\
z\left(n-l_{L-1}\right) e^{-j 2 \pi(L-1) \Delta \nu(m-n)} &
\end{array}\right] \\
& \approx \mathcal{E}\left[\begin{array}{cc}
\frac{1}{1-\lambda / \alpha_{0}^{2}} & \\
\chi \tilde{P}(n) & =R_{c}(n)^{-1} \\
& =\mathcal{E}^{-1}\left[\begin{array}{ll}
1-\lambda / \alpha_{0}^{2} &
\end{array}\right] \\
& \approx(1-\lambda) \mathcal{E}^{-1} I
\end{array}\right]
\end{aligned}
$$

where we have used that $z(n)$ is a white sequence and approximated the expectation with the sample mean. For realistic Doppler spread and symbol rates $\alpha_{i}$ is close to one, e.g., if $B=5 \mathrm{~Hz}$ and $f_{s}=2500 \mathrm{symb} / \mathrm{sec}$ (4.110) yields $\alpha^{2}=0.9875$.

We now turn to the derivation of (4.221). By inserting (4.217) in (4.216) we get

$$
\begin{aligned}
\mathbf{h}_{e}(n) & =\mathbf{h}(n)-\hat{\mathbf{h}}(n) \\
& =\left[I-\chi \tilde{P}(n) \mathbf{c}_{0}(n) \mathbf{c}_{0}^{H}(n)\right]\left[A \mathbf{h}_{e}(n-1)+\mathbf{v}(n)\right]-\chi \tilde{P}(n) \mathbf{c}_{0}(n) w(n) \\
& +\chi \tilde{P}(n) \mathbf{c}_{0}(n) \mathbf{c}_{e}^{H}(n) A\left[\mathbf{h}(n-1)-\mathbf{h}_{e}(n-1)\right]-\chi \tilde{P}(n) \mathbf{c}_{e}(n) \mathbf{c}_{0}^{H}(n) A \mathbf{h}_{e}(n-1) \\
& -\chi \tilde{P}(n) \mathbf{c}_{e}(n) \mathbf{c}_{0}^{H}(n) \mathbf{v}(n)-\chi \tilde{P}(n) \mathbf{c}_{e}(n) w(n) .
\end{aligned}
$$

The error covariance is

$$
\Pi(n)=E\left[\mathbf{h}_{e}(n) \mathbf{h}_{e}^{H}(n)\right] .
$$


In the case of no decision errors $\mathrm{h}_{e}(n)$ is given by the two first terms of (B.9) and in this case it is proven [65] for $A=I$ that $\hat{\Pi}(n)$ given by

$$
\begin{aligned}
\hat{\Pi}(n) & =\left[I-\chi \tilde{P}(n) Q_{0}\right] A \hat{\Pi}(n-1) A^{H}\left[I-\chi \tilde{P}(n) Q_{0}\right]^{H} \\
& +\chi^{2} \tilde{P}(n) Q_{0} \tilde{P}^{H}(n) \sigma_{w}^{2}+R
\end{aligned}
$$

is close to $\Pi(n) . Q_{0}$ is given by (4.222). $\Pi(n)$ can be found by squaring (B.9) and taking expectation, and in order to arrive at (B.11) it is assumed [65] that

$$
\begin{array}{r}
E\left[\tilde{P}(n) \mathbf{c}_{0}(n) \mathbf{c}_{0}^{H}(n) \mathbf{h}_{e}(n) \mathbf{h}_{e}^{H}(n) \mathbf{c}_{0}(n) \mathbf{c}_{0}^{H}(n) \tilde{P}^{H}(n)\right]= \\
\tilde{P}(n) E\left[\mathbf{c}_{0}(n) \mathbf{c}_{0}^{H}(n)\right] E\left[\mathbf{h}_{e}(n) \mathbf{h}_{e}^{H}(n)\right] E\left[\mathbf{c}_{0}(n) \mathbf{c}_{0}^{H}(n)\right] \tilde{P}^{H}(n)
\end{array}
$$

and this assumption will be used herein. We now for brevity omit the time index $n$, and we note that $\mathbf{c}_{0}, \mathbf{c}_{e}, \mathbf{v}, \tilde{P}, w$ are taken at time $n$ and $\mathbf{h}, \mathbf{h}_{e}$ are taken at time $n-1$. By squaring and taking expectation of (B.9) we get (B.14). The equation (B.15) follow from (B.11), and it is the result of the squared expectation of the two first terms in (B.9). The remaining part of (B.14) amounts to carrying out the multiplication of the remaining terms. The equations (B.16)-(B.21) follow by assuming $\hat{\mathbf{h}}, \mathbf{h}_{e}, \mathbf{c}_{e}, \mathbf{c}_{0}, \mathbf{v}, w$ mutually uncorrelated. By means of (4.211) the element $(k, l)$ of the matrix inside the first expectation in (B.17) is

$$
\begin{gathered}
\mathcal{E}^{2} \sum_{i=0}^{L-1} \sum_{j=0}^{L-1} z(n-k) z^{*}(n-i)\left[A \mathbf{h}_{e} \mathbf{h}_{e}^{H} A^{H}\right]_{j i} z_{e}(n-j) z^{*}(n-l) \\
E\left\{\left(\left[I-\chi \tilde{P} \mathbf{c}_{0} \mathbf{c}_{0}^{H}\right]\left[A \mathbf{h}_{e}+\mathbf{v}\right]-\chi \tilde{P} \mathbf{c}_{0} w+\chi \tilde{P} \mathbf{c}_{0} \mathbf{c}_{e}^{H} A\left[\mathbf{h}-\mathbf{h}_{e}\right]-\chi \tilde{P} \mathbf{c}_{e} \mathbf{c}_{0}^{H} A \mathbf{h}_{e}\right.\right. \\
\left.-\chi \tilde{P} \mathbf{c}_{e} \mathbf{c}_{0}^{H} \mathbf{v}-\chi \tilde{P} \mathbf{c}_{e} w\right) \times \\
\left(\left[\mathbf{v}^{H}+\mathbf{h}_{e}^{H} A^{H}\right]\left[I-\chi \mathbf{c}_{0} \mathbf{c}_{0}^{H} \tilde{P}^{H}\right]-\chi \mathbf{c}_{0}^{H} \tilde{P}^{H} w^{*}+\chi\left[\mathbf{h}^{H}-\mathbf{h}_{e}^{H}\right] A^{H} \mathbf{c}_{e} \mathbf{c}_{0}^{H} \tilde{P}^{H}\right. \\
\left.\left.-\chi \mathbf{h}_{e}^{H} A^{H} \mathbf{c}_{0} \mathbf{c}_{e}^{H} \tilde{P}^{H}-\chi \mathbf{v}^{H} \mathbf{c}_{0} \mathbf{c}_{e}^{H} \tilde{P}^{H}-\chi \mathbf{c}_{e}^{H} \tilde{P}^{H} w^{*}\right)\right\}
\end{gathered}
$$




$$
\begin{aligned}
& =\left[I-\chi \tilde{P} Q_{0}\right] A \hat{\Pi} A^{H}\left[I-\chi \tilde{P} Q_{0}\right]^{H}+\chi^{2} \tilde{P} Q_{0} \tilde{P}^{H} \sigma_{w}^{2}+R \\
& +E\left[A \mathbf{h}_{e}\left(\chi\left[\mathbf{h}^{H}-\mathbf{h}_{e}^{H}\right] A^{H} \mathbf{c}_{e} \mathbf{c}_{0}^{H} \tilde{P}^{H}-\chi \mathbf{h}_{e}^{H} A^{H} \mathbf{c}_{0} \mathbf{c}_{e}^{H} \tilde{P}^{H}-\chi \mathbf{v}^{H} \mathbf{c}_{0} \mathbf{c}_{e}^{H} \tilde{P}^{H}-\chi \mathbf{c}_{e}^{H} \tilde{P}^{H} w^{*}\right)\right. \\
& \text { - } \chi \tilde{P} E\left[\mathbf { c } _ { 0 } \mathbf { c } _ { 0 } ^ { H } A \mathbf { h } _ { e } \left(\chi\left[\mathbf{h}^{H}-\mathbf{h}_{e}^{H}\right] A^{H} \mathbf{c}_{e} \mathbf{c}_{0}^{H} \tilde{P}^{H}-\chi \mathbf{h}_{e}^{H} A^{H} \mathbf{c}_{0} \mathbf{c}_{e}^{H} \tilde{P}^{H}-\chi \mathbf{v}^{H} \mathbf{c}_{0} \mathbf{c}_{e}^{H} \tilde{P}^{H}\right.\right. \\
& \left.\left.-\chi \mathbf{c}_{e}^{H} \tilde{P}^{H} w^{*}\right)\right] \\
& +E\left[( I - \chi \tilde { P } \mathbf { c } _ { 0 } \mathbf { c } _ { 0 } ^ { H } ) \mathbf { v } \left(\chi\left[\mathbf{h}^{H}-\mathbf{h}_{e}^{H}\right] A^{H} \mathbf{c}_{e} \mathbf{c}_{0}^{H} \tilde{P}^{H}-\chi \mathbf{h}_{e}^{H} A^{H} \mathbf{c}_{0} \mathbf{c}_{e}^{H} \tilde{P}^{H}-\chi \mathbf{v}^{H} \mathbf{c}_{0} \mathbf{c}_{e}^{H} \tilde{P}^{H}\right.\right. \\
& \text { - } \left.\left.\chi \mathbf{c}_{e}^{H} \tilde{P}^{H} w^{*}\right)\right] \\
& -\chi \tilde{P} E\left[\mathbf { c } _ { 0 } w \left(\chi\left[\mathbf{h}^{H}-\mathbf{h}_{e}^{H}\right] A^{H} \mathbf{c}_{e} \mathbf{c}_{0}^{H} \tilde{P}^{H}-\chi \mathbf{h}_{e}^{H} A^{H} \mathbf{c}_{0} \mathbf{c}_{e}^{H} \tilde{P}^{H}-\chi \mathbf{v}^{H} \mathbf{c}_{0} \mathbf{c}_{e}^{H} \tilde{P}^{H}\right.\right. \\
& \left.\left.-\chi \mathbf{c}_{e}^{H} \tilde{P}^{H} w^{*}\right)\right] \\
& +\chi \tilde{P} E\left[\mathbf { c } _ { 0 } \mathbf { c } _ { e } ^ { H } A [ \mathbf { h } - \mathbf { h } _ { e } ] \left(\chi\left[\mathbf{h}^{H}-\mathbf{h}_{e}^{H}\right] A^{H} \mathbf{c}_{e} \mathbf{c}_{0}^{H} \tilde{P}^{H}-\chi \mathbf{h}_{e}^{H} A^{H} \mathbf{c}_{0} \mathbf{c}_{e}^{H} \tilde{P}^{H}-\chi \mathbf{v}^{H} \mathbf{c}_{0} \mathbf{c}_{e}^{H} \tilde{P}^{H}\right.\right. \\
& \left.\left.-\chi \mathbf{c}_{e}^{H} \tilde{P}^{H} w^{*}\right)\right] \\
& -\chi \tilde{P} E\left[\mathbf { c } _ { e } \mathbf { c } _ { 0 } ^ { H } ( A \mathbf { h } _ { e } + \mathbf { v } ) \left(\chi\left[\mathbf{h}^{H}-\mathbf{h}_{e}^{H}\right] A^{H} \mathbf{c}_{e} \mathbf{c}_{0}^{H} \tilde{P}^{H}-\chi \mathbf{h}_{e}^{H} A^{H} \mathbf{c}_{0} \mathbf{c}_{e}^{H} \tilde{P}^{H}-\chi \mathbf{v}^{H} \mathbf{c}_{0} \mathbf{c}_{e}^{H} \tilde{P}^{H}\right.\right. \\
& \text { - } \left.\left.\chi \mathbf{c}_{e}^{H} \tilde{P}^{H} w^{*}\right)\right] \\
& -\chi \tilde{P} E\left[\mathbf { c } _ { e } w \left(\chi\left[\mathbf{h}^{H}-\mathbf{h}_{e}^{H}\right] A^{H} \mathbf{c}_{e} \mathbf{c}_{0}^{H} \tilde{P}^{H}-\chi \mathbf{h}_{e}^{H} A^{H} \mathbf{c}_{0} \mathbf{c}_{e}^{H} \tilde{P}^{H}-\chi \mathbf{v}^{H} \mathbf{c}_{0} \mathbf{c}_{e}^{H} \tilde{P}^{H}\right.\right. \\
& \left.\left.-\chi \mathbf{c}_{e}^{H} \tilde{P}^{H} w^{*}\right)\right] \\
& =\left[I-\chi \tilde{P} Q_{0}\right] A \hat{\Pi} A^{H}\left[I-\chi \tilde{P} Q_{0}\right]^{H}+\chi^{2} \tilde{P} Q_{0} \tilde{P}^{H} \sigma_{w}^{2}+R \\
& +0 \\
& \text { - } \chi^{2} \tilde{P} E\left[\mathbf{c}_{0} \mathbf{c}_{0}^{H} A \mathbf{h}_{e} \mathbf{h}_{e}^{H} A^{H} \mathbf{c}_{e} \mathbf{c}_{0}^{H}\right] \tilde{P}^{H}+\chi^{2} \tilde{P} E\left[\mathbf{c}_{0} \mathbf{c}_{0}^{H} A \mathbf{h}_{e} \mathbf{h}_{e}^{H} A^{H} \mathbf{c}_{0} \mathbf{c}_{e}^{H}\right] \tilde{P}^{H} \\
& +\chi^{2} \tilde{P} E\left[\mathbf{c}_{0} \mathbf{c}_{0}^{H} \mathbf{v} \mathbf{v}^{H} A^{H} \mathbf{c}_{0} \mathbf{c}_{e}^{H}\right] \tilde{P}^{H} \\
& -0 \\
& +\chi^{2} \tilde{P} E\left[\mathbf{c}_{0} \mathbf{c}_{e}^{H} A\left(\mathbf{h h}^{H}+\mathbf{h}_{e} \mathbf{h}_{e}^{H}\right) A^{H} \mathbf{c}_{e} \mathbf{c}_{0}^{H}\right] A^{H} \tilde{P}^{H}-\chi^{2} \tilde{P} \times \\
& E\left[\mathrm{c}_{0} \mathrm{c}_{e}^{H} A \mathbf{h}_{e} \mathrm{~h}_{e}^{H} A^{H} \mathbf{c}_{0} \mathrm{c}_{e}^{H}\right] \tilde{P}^{H} \\
& +\chi^{2} \tilde{P} E\left[\mathbf{c}_{e} \mathbf{c}_{0}^{H} A \mathbf{h}_{e} \mathbf{h}_{e}^{H} A^{H} \mathbf{c}_{e} \mathbf{c}_{0}^{H}\right] A^{H} \tilde{P}^{H}+\chi^{2} \tilde{P} E\left[\mathbf{c}_{e} \mathbf{c}_{0}^{H} A \mathbf{h}_{e} \mathbf{h}_{e}^{H} A^{H} \mathbf{c}_{0} \mathbf{c}_{e}^{H}\right] \tilde{P}^{H} \\
& +\chi^{2} \tilde{P} E\left[\mathbf{c}_{e} \mathbf{c}_{0}^{H} \mathbf{v v}^{H} \mathbf{c}_{0} \mathbf{c}_{e}^{H}\right] \tilde{P}^{H} \\
& +\chi^{2} \tilde{P} E\left[\mathbf{c}_{e} \mathbf{c}_{e}^{H} w w^{*}\right] \tilde{P}^{H}
\end{aligned}
$$


By taking expectation of (B.13), and remembering that $z(n)$ and $z_{e}(n)$ are white and mutually uncorrelated, all terms where $k=l=i$ is not satisfied are zero. From (4.202) and the assumption that all values of $z(n)$ are equally likely we have for the case $k=l=i$ that

$$
E\left[z(n) z(n) z^{*}(n)\right]=\frac{1}{4}\left(j^{2}(-j)+(-1)^{3}+1^{3}+(-j)^{2} j\right)=0 .
$$

Thus the first term of (B.17) is zero. The element $(k, l)$ of the matrix inside the second expectation of (B.17) is the conjugate of (B.13) therefore this term is also zero. The only difference between (B.18) and the second term of (B.17) is the middle matrix $\mathbf{v} \mathbf{v}^{H}$, but since this is uncorrelated with $\mathbf{c}_{e}$ and $\mathbf{c}_{0}$ this expectation is also zero by means of (B.13). Likewise the element $(k, l)$ of the matrix inside the first expectation of (B.20) is

$$
\mathcal{E}^{2} \sum_{i=0}^{L-1} \sum_{j=0}^{L-1} z(n-k) z_{e}^{*}(n-i)\left[A\left(\mathbf{h h}^{H}+\mathbf{h}_{e} \mathbf{h}_{e}^{H}\right) A^{H}\right]_{j i} z_{e}(n-j) z^{*}(n-l) .
$$

Taking expectation of (B.25) we get

$$
\begin{aligned}
& \mathcal{E}^{2} \sum_{i=0}^{L-1} \sum_{j=0}^{L-1} E\left[z(n-k) z^{*}(n-l)\right] E\left[\left[A\left(\mathrm{hh}^{H}+\mathbf{h}_{e} \mathbf{h}_{e}^{H}\right) A^{H}\right]_{j i}\right] E\left[z_{e}(n-j) z_{e}^{*}(n-i)\right] \\
= & \mathcal{E}^{2} \sum_{i=0}^{L-1} \sum_{j=0}^{L-1} \delta(k-l) \delta(j-i) E\left[\left[A\left(\mathbf{h h}^{H}+\mathbf{h}_{e} \mathbf{h}_{e}^{H}\right) A^{H}\right]_{j i}\right] E\left[\left|z_{e}\right|^{2}\right] \\
= & \mathcal{E}^{2} \gamma P_{e} \delta(k-l) \sum_{i=0}^{L-1} E\left[\left[A\left(\mathbf{h h}^{H}+\mathbf{h}_{e} \mathbf{h}_{e}^{H}\right) A^{H}\right]_{i i}\right] \\
= & \mathcal{E}^{2} \gamma P_{e} \delta(k-l) \operatorname{tr}\left(E\left[A\left(\mathbf{h h}^{H}+\mathbf{h}_{e} \mathbf{h}_{e}^{H}\right) A^{H}\right]\right)
\end{aligned}
$$

where we have used (4.207) and $\gamma=8 / 3$. The element $(k, l)$ of the matrix inside the second expectation of (B.20) is

$$
\mathcal{E}^{2} \sum_{i=0}^{L-1} \sum_{j=0}^{L-1} z(n-k) z_{e}^{*}(n-i)\left[A \mathrm{~h}_{e} \mathrm{~h}_{e}^{H} A^{H}\right]_{j i} z_{e}^{*}(n-l) z(n-j) .
$$


Taking expectation yields zero for all terms where $k=j$ and $i=l$ is not satisfied. The rest of the terms in (B.27) are of the form

$$
\mathcal{E}^{2} E\left[z^{2}(n-k)\right] E\left[z_{e}^{* 2}(n-l)\right] E\left[\left[A \mathbf{h}_{e} \mathbf{h}_{e}^{H} A^{H}\right]_{j i}\right]
$$

and since

$$
E\left[z^{2}(n-k)\right]=\frac{1}{4}\left((-j)^{2}+(-1)^{2}+1^{2}+j^{2}\right)=0
$$

they are also zero. The first term of (B.21) is the hermitian transpose of the second term of (B.20) and thus it is zero. The second term of (B.21) is of similar form as the first term of (B.20) and it is given by (B.26). The matrix inside the expectation of (B.22) is the same as the first term of (B.20) when we replace $A\left(\mathbf{h} \mathbf{h}^{H}+\mathbf{h}_{e} \mathbf{h}_{e}^{H}\right) A^{H}$ with $\mathbf{v v}^{H}$. Thus (B.26) yields

$$
\mathcal{E}^{2} \gamma P_{e} \delta(l-k) \operatorname{tr}\left(\mathbf{v v}^{H}\right)
$$

for element $(l, k)$ of this term. Thus we have from (B.15)-(B.23),(B.26) that

$$
\begin{aligned}
\hat{\Pi}(n) & =\left[I-\chi \tilde{P}(n) Q_{0}\right] A \hat{\Pi}(n-1) A^{H}\left[I-\chi \tilde{P}(n) Q_{0}\right]^{H} \\
& +\chi^{2} \tilde{P}(n) Q_{0} \tilde{P}^{H}(n) \sigma_{w}^{2}+R \\
& +\chi^{2} \tilde{P}(n) \mathcal{E}^{2} \gamma P_{e}\left(\operatorname{tr}\left(E\left[A \mathbf{h}(n-1) \mathbf{h}^{H}(n-1) A^{H}\right]\right)+2 \operatorname{tr}\left(A \hat{\Pi}(n-1) A^{H}\right)\right) \tilde{P}^{H}(n) \\
& +\chi^{2} \tilde{P}(n) \mathcal{E}^{2} \gamma P_{e} \operatorname{tr}(R) \tilde{P}^{H}(n)+\chi^{2} \tilde{P}(n) Q_{e} \sigma_{w}^{2} \tilde{P}^{H}(n) .
\end{aligned}
$$

The channel is modeled as a normalized AR(1) process so that

$$
E\left[\mathrm{hh}^{H}\right]=\operatorname{diag}\left(\left[u_{0}^{2} \cdots u_{L-1}^{2}\right]\right)
$$


and using (B.32) in (B.31) an rearranging we get

$$
\begin{aligned}
\hat{\Pi}(n) & =\left[I-\chi \tilde{P}(n) Q_{0}\right] A \hat{\Pi}(n-1) A^{H}\left[I-\chi \tilde{P}(n) Q_{0}\right]^{H} \\
& +\chi^{2} \tilde{P}(n) Q_{0} \tilde{P}^{H}(n) \sigma_{w}^{2}+R \\
& +\chi^{2} \tilde{P}(n) \mathcal{E}^{2} \gamma P_{e}\left(L+2 \operatorname{tr}\left(A \hat{\Pi}(n-1) A^{H}\right)\right) \tilde{P}^{H}(n) \\
& +\chi^{2} \tilde{P}(n) Q_{e} \sigma_{w}^{2} \tilde{P}^{H}(n) .
\end{aligned}
$$




\section{Appendix C}

\section{Approximations in computing the hypothesis probability}

The purpose of this appendix is to motivate the approximation of (4.167) by (4.168), and the omission of the real term in (4.174).

Prerequisites The Gaussian product-moment theorem: Assume $x_{i}$ jointly Gaussian, zero-mean. Then

$$
E\left[x_{1} x_{2} x_{3} x_{4}\right]=E\left[x_{1} x_{2}\right] E\left[x_{3} x_{4}\right]+E\left[x_{1} x_{3}\right] E\left[x_{2} x_{4}\right]+E\left[x_{1} x_{4}\right] E\left[x_{2} x_{3}\right]
$$

We use an $\mathrm{AR}(1)$ model for $U_{l, k}(m)$ (4.86) driven with Gaussian white noise, therefore $U_{l, k}(m)$ is a Gaussian random process:

$$
U_{l, k}(m) \sim N\left(0, u_{l, k}^{2}\right) .
$$

The WSSUS assumption yields

$$
E\left[U_{l_{1}, k_{1}}\left(m_{1}\right) U_{l_{2}, k_{2}}^{*}\left(m_{2}\right)\right]=u_{l_{1}, k_{1}}^{2} \delta\left(l_{1}-l_{2}\right) \delta\left(k_{1}-k_{2}\right) .
$$


For notational purposes define

$$
a(l, o, m)=z(m) z^{*}(m-l) U_{l, o}^{*}(m) e^{-j 2 \pi o \Delta \nu m} .
$$

From (C.3) and (C.4) we have that

$$
\begin{aligned}
a(l, o, m) & \sim N\left(0, u_{l, o}^{2}\right) \\
E\left[a\left(l_{1}, o_{1}, m_{1}\right) a^{*}\left(l_{2}, o_{2}, m_{2}\right)\right] & =z\left(m_{1}\right) z^{*}\left(m_{1}-l_{1}\right) z\left(m_{2}\right) z^{*}\left(m_{2}-l_{1}\right) \\
& \times u_{l_{1}, o_{1}}^{2} e^{-j 2 \pi o_{1} \Delta \nu\left(m_{1}-m_{2}\right)} \delta\left(l_{1}-l_{2}\right) \\
& \times \delta\left(o_{1}-o_{2}\right) .
\end{aligned}
$$

Approximation of $\theta_{1}$ We want to justify the approximation of (4.167) by (4.168), here restated as (C.6) and (C.7).

$$
\begin{aligned}
\theta_{1} & =\mathcal{E}^{2}\left|\sum_{(l, o)} \sum_{m=0}^{M-1} z(m) z^{*}(m-l) U_{l, o}^{*}(m) e^{-j 2 \pi o \Delta \nu m}\right|^{2} \\
& =\mathcal{E}^{2}\left|\sum_{(l, o)} \sum_{m=0}^{M-1} a(l, o, m)\right|^{2} \\
\theta_{1}^{\prime} & =\mathcal{E}^{2} \sum_{(l, o)}\left|\sum_{m=0}^{M-1} z(m) z^{*}(m-l) U_{l, o}^{*}(m) e^{-j 2 \pi o \Delta \nu m}\right|^{2} \\
& =\mathcal{E}^{2} \sum_{(l, o)}\left|\sum_{m=0}^{M-1} a(l, o, m)\right|^{2}
\end{aligned}
$$

where we use $\theta_{1}^{\prime}$ in (C.7) to distinguish the approximation.

Claim: The first and second order moment of $\theta_{1}$ and $\theta_{1}^{\prime}$ from (C.6) and (C.7) are equal.

Intuitive argument: The impulsive second order statistics of (C.5) enable us to move $\sum_{(l, o)}$ outside the squaring operation in (C.6) when taking expectation. 
Formal argument: Compute the moments. Using (C.5) in (C.6) yields

$$
\begin{aligned}
E\left[\theta_{1}\right] & =\mathcal{E}^{2} \sum_{\left(l_{1}, o_{1}\right)} \sum_{\left(l_{2}, o_{2}\right)} \sum_{m_{1}=0}^{M-1} \sum_{m_{2}=0}^{M-1} E\left[a\left(l_{1}, o_{1}, m_{1}\right) a^{*}\left(l_{2}, o_{2}, m_{2}\right)\right] \\
& =\left.\mathcal{E}^{2} \sum_{(l, o)} u_{l, o}^{2} \sum_{m=0}^{M-1} z(m) z^{*}(m-l) e^{-j 2 \pi o \Delta \nu m}\right|^{2} \\
& \approx(M \mathcal{E})^{2} u_{0,0}^{2}+M \mathcal{E}^{2} \sum_{(l, o) \neq(0,0)} u_{l, o}^{2} .
\end{aligned}
$$

Using (C.5) in (C.7) yields

$$
\begin{aligned}
E\left[\theta_{1}^{\prime}\right] & =\mathcal{E}^{2} \sum_{(l, o)} \sum_{m_{1}=0}^{M-1} \sum_{m_{2}=0}^{M-1} E\left[a\left(l, o, m_{1}\right) a^{*}\left(l, o, m_{2}\right)\right] \\
& =\mathcal{E}^{2} \sum_{(l, o)} u_{l, o}^{2}\left|\sum_{m=0}^{M-1} z(m) z^{*}(m-l) e^{-j 2 \pi o \Delta \nu m}\right|^{2} .
\end{aligned}
$$

Using (C.5) and (C.1) in the squared version of (C.6) yields

$$
\begin{aligned}
E\left[\theta_{1}^{2}\right] & =\mathcal{E}^{4} E\left[\left|\sum_{(l, o)} \sum_{m=0}^{M-1} a(l, o, m)\right|^{4}\right] \\
& =\mathcal{E}^{4} E\left[\sum_{\left(l_{1}, o_{1}\right)} \sum_{m_{1}=0}^{M-1} \sum_{\left(l_{2}, o_{2}\right)} \sum_{m_{2}=0}^{M-1} \sum_{\left(l_{3}, o_{3}\right)} \sum_{m_{3}=0}^{M-1} \sum_{\left(l_{4}, o_{4}\right)} \sum_{m_{4}=0}^{M-1} a\left(l_{1}, o_{1}, m_{1}\right) a^{*}\left(l_{2}, o_{2}, m_{2}\right)\right. \\
& \left.\times a\left(l_{3}, o_{3}, m_{3}\right) a^{*}\left(l_{4}, o_{4}, m_{4}\right)\right] \\
& =\mathcal{E}^{4} \sum_{\left(l_{1}, o_{1}\right)} \sum_{m_{1}=0}^{M-1} \sum_{\left(l_{2}, o_{2}\right)} \sum_{m_{2}=0}^{M-1} \sum_{\left(l_{3}, o_{3}\right)} \sum_{m_{3}=0}^{M-1} \sum_{\left(l_{4}, o_{4}\right)} \sum_{m_{4}=0}^{M-1}\left(E\left[a\left(l_{1}, o_{1}, m_{1}\right) a^{*}\left(l_{2}, o_{2}, m_{2}\right)\right]\right. \\
& \times E\left[a\left(l_{3}, o_{3}, m_{3}\right) a^{*}\left(l_{4}, o_{4}, m_{4}\right)\right] \\
& +E\left[a\left(l_{1}, o_{1}, m_{1}\right) a\left(l_{3}, o_{3}, m_{3}\right)\right] E\left[a^{*}\left(l_{2}, o_{2}, m_{2}\right) a^{*}\left(l_{4}, o_{4}, m_{4}\right)\right] \\
& \left.+E\left[a\left(l_{1}, o_{1}, m_{1}\right) a^{*}\left(l_{4}, o_{4}, m_{4}\right)\right] E\left[a^{*}\left(l_{2}, o_{2}, m_{2}\right) a\left(l_{3}, o_{3}, m_{3}\right)\right]\right) \\
& =2 \mathcal{E}^{4} \sum_{\left(l_{1}, o_{1}\right)} \sum_{m_{1}=0}^{M-1} \sum_{\left(l_{2}, o_{2}\right)}^{M-1} \sum_{m_{2}=0}^{M-1} E\left[a\left(l_{1}, o_{1}, m_{1}\right) a^{*}\left(l_{2}, o_{2}, m_{2}\right)\right] \\
& \times \sum_{\left(l_{3}, o_{3}\right)} \sum_{m_{3}=0}^{M-1} \sum_{\left(l_{4}, o_{4}\right)} \sum_{m_{4}=0}^{M-1} E\left[a\left(l_{3}, o_{3}, m_{3}\right) a^{*}\left(l_{4}, o_{4}, m_{4}\right)\right]
\end{aligned}
$$




$$
\begin{aligned}
& =2 \mathcal{E}^{4}\left(\sum_{(l, o)} u_{l, o}^{2} \sum_{m_{1}=0}^{M-1} \sum_{m_{2}=0}^{M-1} z\left(m_{1}\right) z^{*}\left(m_{1}-l\right) z^{*}\left(m_{2}\right) z\left(m_{2}-l\right) e^{-j 2 \pi o \Delta \nu\left(m_{1}-m_{2}\right)}\right)^{2} \\
& =2 E\left[\theta_{1}\right]^{2} \approx 2\left[\left(M \mathcal{E} u_{0,0}\right)^{4}+2 M^{3} \mathcal{E}^{4} \sum_{(l, o)} u_{l, o}^{2}+M^{2} \mathcal{E}^{4}\left(\sum_{(l, o)} u_{l, o}^{2}\right)^{2}\right] .
\end{aligned}
$$

Using (C.5) and (C.1) in the squared version of (C.7) yields

$$
\begin{aligned}
& E\left[\theta_{1}^{\prime 2}\right]=\mathcal{E}^{4} E\left[\sum_{\left(l_{1}, o_{1}\right)}\left|\sum_{m_{1}=0}^{M-1} a\left(l_{1}, o_{1}, m_{1}\right)\right|^{2} \sum_{\left(l_{2}, o_{2}\right)}\left|\sum_{m_{2}=0}^{M-1} a\left(l_{2}, o_{2}, m_{2}\right)\right|^{2}\right] \\
& =\mathcal{E}^{4} \sum_{\left(l_{1}, o_{1}\right)} \sum_{\left(l_{2}, o_{2}\right)} E\left[\sum_{m_{1}=0}^{M-1} \sum_{m_{2}=0}^{M-1} a\left(l_{1}, o_{1}, m_{1}\right) a^{*}\left(l_{1}, o_{1}, m_{2}\right)\right. \\
& \left.\times \sum_{m_{3}=0}^{M-1} \sum_{m_{4}=0}^{M-1} a\left(l_{2}, o_{2}, m_{3}\right) a^{*}\left(l_{2}, o_{2}, m_{4}\right)\right] \\
& =\mathcal{E}^{4} \sum_{\left(l_{1}, o_{1}\right)} \sum_{\left(l_{2}, o_{2}\right)} \sum_{m_{1}=0}^{M-1} \sum_{m_{2}=0}^{M-1} \sum_{m_{3}=0}^{M-1} \sum_{m_{4}=0}^{M-1} E\left[a\left(l_{1}, o_{1}, m_{1}\right) a^{*}\left(l_{1}, o_{1}, m_{2}\right)\right. \\
& \left.\times \quad a\left(l_{2}, o_{2}, m_{3}\right) a^{*}\left(l_{2}, o_{2}, m_{4}\right)\right] \\
& =\mathcal{E}^{4} \sum_{\left(l_{1}, o_{1}\right)} \sum_{\left(l_{2}, o_{2}\right)} \sum_{m_{1}=0}^{M-1} \sum_{m_{2}=0}^{M-1} \sum_{m_{3}=0}^{M-1} \sum_{m_{4}=0}^{M-1}\left(E\left[a\left(l_{1}, o_{1}, m_{1}\right) a^{*}\left(l_{1}, o_{1}, m_{2}\right)\right]\right. \\
& \times E\left[a\left(l_{2}, o_{2}, m_{3}\right) a^{*}\left(l_{2}, o_{2}, m_{4}\right)\right] \\
& +E\left[a\left(l_{1}, o_{1}, m_{1}\right) a\left(l_{2}, o_{2}, m_{3}\right)\right] E\left[a^{*}\left(l_{1}, o_{1}, m_{2}\right) a^{*}\left(l_{2}, o_{2}, m_{4}\right)\right] \\
& \left.+E\left[a\left(l_{1}, o_{1}, m_{1}\right) a^{*}\left(l_{2}, o_{2}, m_{4}\right)\right] E\left[a^{*}\left(l_{1}, o_{1}, m_{2}\right) a\left(l_{2}, o_{2}, m_{3}\right)\right]\right) \\
& =2 \mathcal{E}^{4} \sum_{\left(l_{1}, o_{1}\right)} \sum_{m_{1}=0}^{M-1} \sum_{m_{2}=0}^{M-1} E\left[a\left(l_{1}, o_{1}, m_{1}\right) a^{*}\left(l_{1}, o_{1}, m_{2}\right)\right] \\
& \times \sum_{\left(l_{2}, o_{2}\right)} \sum_{m_{3}=0}^{M-1} \sum_{m_{4}=0}^{M-1} E\left[a\left(l_{2}, o_{2}, m_{3}\right) a^{*}\left(l_{2}, o_{2}, m_{4}\right)\right] \\
& =2 \mathcal{E}^{4}\left(\sum_{(l, o)} u_{l, o}^{2} \sum_{m_{1}=0}^{M-1} \sum_{m_{2}=0}^{M-1} z\left(m_{1}\right) z^{*}\left(m_{1}-l\right) z^{*}\left(m_{2}\right) z\left(m_{2}-l\right) e^{-j 2 \pi o \Delta \nu\left(m_{1}-m_{2}\right)}\right)^{2} \\
& =2 \mathcal{E}^{4}\left(\sum_{(l, o)} u_{l, o}^{2}\left|\sum_{m=0}^{M-1} z(m) z^{*}(m-l) e^{-j 2 \pi \circ \Delta \nu m}\right|^{2}\right)^{2} \\
& =2 E\left[\theta_{1}^{\prime}\right]^{2} \text {. }
\end{aligned}
$$


By comparing the first and second order moments of $\theta_{1}$ and $\theta_{1}^{\prime}$ they are found to be identical.

Approximation of $\theta_{2}$ We now turn to the cross-term in (4.174)

$$
\begin{aligned}
\theta_{2}= & 2 \mathcal{E}^{3 / 2} \operatorname{Re}\left[\sum_{(l, o)} U_{l, o}^{*} \sum_{m=0}^{M-1} z(m) z^{*}(m-l) e^{-j 2 \pi o \Delta \nu m} \times\right. \\
& \left.\sum_{m=0}^{M-1} z(m) w^{*}(m)\right] \\
= & 2 \mathcal{E}^{3 / 2} \operatorname{Re}\left[\sum_{(l, o)} \sum_{m=0}^{M-1} a(l, o, m) \sum_{m=0}^{M-1} z(m) w^{*}(m)\right] .
\end{aligned}
$$

The $R e$ operator is expressed by

$$
\begin{aligned}
2 \operatorname{Re}[x] & =x+x^{*} \\
4(\operatorname{Re}[x])^{2} & =x^{2}+2|x|^{2}+\left(x^{*}\right)^{2} .
\end{aligned}
$$

We have that

$$
\begin{aligned}
E\left[a\left(l_{1}, o_{1}, m_{1}\right) a\left(l_{2}, o_{2}, m_{2}\right)\right] & =E\left[a^{*}\left(l_{1}, o_{1}, m_{1}\right) a^{*}\left(l_{2}, o_{2}, m_{2}\right)\right] \\
E\left[w\left(m_{1}\right) w\left(m_{2}\right)\right] & =E\left[w^{*}\left(m_{1}\right) w^{*}\left(m_{2}\right)\right]=0 .
\end{aligned}
$$

By means of (C.12), (C.13) and (C.14) the first and second moment of $\theta_{2}$ are

$$
\begin{aligned}
E\left[\theta_{2}\right] & =0 \\
E\left[\theta_{2}^{2}\right] & =2 \mathcal{E}^{3} E\left[\left|\sum_{(l, o)} \sum_{m=0}^{M-1} a(l, o, m) \sum_{m=0}^{M-1} z(m) w^{*}(m)\right|^{2}\right] \\
& =2 \mathcal{E}^{3} E\left[\left|\sum_{(l, o)} \sum_{m=0}^{M-1} a(l, o, m)\right|^{2}\right] E\left[\left|\sum_{m=0}^{M-1} z(m) w^{*}(m)\right|^{2}\right] \\
& =2 M \mathcal{E} \sigma_{w}^{2} E\left[\theta_{1}\right] \approx 2 \sigma_{w}^{2}\left[(M \mathcal{E})^{3} u_{0,0}^{2}+M^{2} \mathcal{E}^{3} \sum_{(l, o) \neq(0,0)} u_{l, o}^{2}\right] .
\end{aligned}
$$


The rough shape of the probability densities of $\theta_{1}$ and $\theta_{2}$ are given by their first and second order moments. The ratio $E\left[\theta_{1}\right] / \sqrt{E\left[\theta_{2}^{2}\right]}$ is 22 for $M=512$. The scenario is outlined in Fig. C-1. This roughly means that the random variable $\theta_{2}$ must exceed 22 times its standard deviation to impact the sum $\theta_{1}+\theta_{2}$ severely. This event has very low probability, and thus $\theta_{2}$ is neglected.

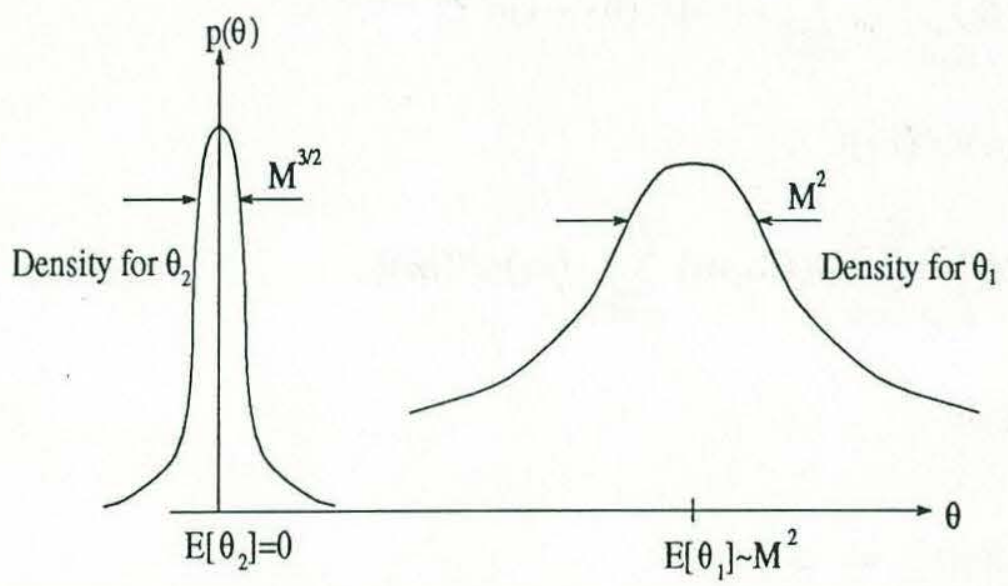

Figure C-1: Sketch of the probability densities of $\theta_{1}$ and $\theta_{2}$. 


\section{Bibliography}

[1] K. Aki. Quantitative seismology. W.H. Freeman, 1980.

[2] Al-Hussaini and Kassam. Robust eckart filters for time delay estimation. IEEE Trans. on Acoustics Speech and Signal Processing, pages 1052-, October 1984.

[3] Altekar and Beaulieu. Upper bounds to the error probability of a decision feedback equalizer. IEEE Trans. on Inf.Theory, January 1993.

[4] L. Andriot, G. Tziritas, and G. Jourdain. Discrete realization for receivers detecting signals over random dispersive channels. part 2: Doppler-spread channels. Signal processing, pages 89-100, September 1985.

[5] M. Athans. 6.233 multivariable control system I. Supplementary course notes, MIT 1994.

[6] A.B. Baggeroer. Acoustic telemetry - an overview. IEEE Journal of Ocean Engineering, pages 229-235, October 1984.

[7] P.A. Bello. Characterization of randomly time-variant linear channels. IEEE Trans. on Information Theory, pages 360-393, December 1963.

[8] P.A. Bello. Time-frequency duality. IEEE Trans. on Information Theory, pages 18-33, January 1964.

[9] J.A.C. Bingham. Multicarrier modulation for data transmission: An idea which time has come. IEEE communications magazine, pages 5-14, May 1990. 
[10] R.E. Blahut. Radar and sonar. Springer Verlag, 1991.

[11] K.F. Bowden. Physical oceanography of coastal waters. Ellis Horwood limited, 1983.

[12] Bragard and Jourdain. Adaptive equalization for underwater data transmission. In ICASSP, pages 1171-, 1989.

[13] D.H. Brandwood. A complex gradient operator and its application in adaptive array theory. IEE Proceedings Pts. F and $H$, pages 11-16, February 1983.

[14] L.M. Brekhovskikh and Y.P Lysanov. Fundamentals of ocean acoustics. Springer Verlag, 1990.

[15] V. Capellano, G. Loubet, and G. Jourdain. Adaptive multichannel equalizer for underwater communication. In Proc. Oceans'96, 1996.

[16] Tremblay Carter and Lytle. A practical approach to the estimation of amplitude and time-delay parameters of a composite signal. IEEE Journal of Ocean Engineering, pages 273-, January 1987.

[17] J. Catipovic. Design and performance of a digital acoustic telemetry system for the short range underwater channel. IEEE Journal of Ocean Engineering, October 1984 .

[18] J. Catipovic. Spatial diversity processing for underwater acoustic telemetry. IEEE Journal of Ocean Engineering, January 1991.

[19] J. Catipovic and A.B. Baggeroer. Performance of sequential decoding of convolutional codes over fully fading ocean acoustic channels. IEEE Journal of Ocean Engineering, pages 1-7, January 1990. 
[20] J. Catipovic, M. Deffenbaugh, L. Freitag, and D. Frye. An acoustic telemetry system for deep ocean mooring data acquisition and control. In Proc. Oceans'89, pages 887-892, October 1989.

[21] Ching and Chan. Constrained adaptation for time delay estimation with multipath propagation. IEE Radar and signal processing, pages 453-, October 1991.

[22] C.S. Clay and H. Medwin. Acoustical oceanography. Wiley, 1977.

[23] C.S. Clay, H. Medwin, and W.M Wright. Specularly scattered sound and the probability density of a rough surface. J. Acoust. Soc. Am., pages 1677-1682, 1973.

[24] R.W. Coates. A deep ocean penetrator telemetry system. IEEE Journal of Ocean Engineering, April 1988.

[25] M.D. Collins and W.A. Kuperman. Overcoming ray chaos. J. Acoust. Soc. Am., pages 3167-3170, June 1994.

[26] Doherty. Decision feedback equalization of data with spectral nulls. In Military Communication Conf., pages 76-, 1990.

[27] D.R. Dowling and D.R. Jackson. Coherence of acoustic scattering from a dynamic rough surface. J. Acoust. Soc. Am., pages 3149-3157, June 1993.

[28] T. Duda. Modeling weak fluctuations of undersea telemetry signals. IEEE Journal of Ocean Engineering, pages 3-12, January 1991.

[29] I. Dyer. 13.851 fundamentals and applications of underwater sound. Supplementary course notes, MIT 1995.

[30] C. Eckart. Scattering of sound from the sea surface. J. Acoust. Soc. Am., 1953. 
[31] E. Eleftheriou and D.D. Falconer. Tracking properties and steady state performance of RLS adaptive filter algorithms. IEEE Transactions on acoustics speech and signal processing, pages 1097-1109, October 1986.

[32] Etter and Cheng. System modeling using an adaptive delay filter. IEEE Trans. on Circuits and Systems, pages 770-, July 1987.

[33] A. Falahati. Underwater acoustic channel models for $4800 \mathrm{~b} / \mathrm{s}$ QPSK signals. IEEE Journal of Ocean Engineering, pages 12-21, January 1991.

[34] D.D. Falconer. Analysis of a gradient algorithm for simultaneous passband equalization and carrier phase recovery. Bell Systems Technical Journal, pages 409-428, April 1976.

[35] D.D. Falconer. Jointly adaptive equalization and carrier recovery in twodimensional digital communication systems. Bell Systems Technical Journal, pages 317-334, March 1976.

[36] J. Fischer. A high rate, underwater acoustic data communications transceiver. In Proc. Oceans'92, pages 571-576, 1992.

[37] S. Flatte. Sound transmission through a fluctuating ocean. Cambridge University Press, 1979.

[38] L. Freitag and S. Merriam. Robust 5000 bit per second underwater communication system for remote applications. In Proc. MTS '90 Conf., February 1990.

[39] G.V. Frisk. Ocean and Seabed Acoustics. Prentice-Hall, 1994.

[40] R.G. Gallager. Information theory and reliable communication. Wiley, 1968. 
[41] Gazanhes. Signal processing for the multimodal transmission in shallow water propagation; application to underwater acoustic communications. Eurasip Signal Processing Proceedings, June 1984.

[42] Georgiades. Optimum delay and sequence estimation for incomplete data. IEEE Trans. on Acoustics Speech and Signal Processing, pages 202-, January 1990.

[43] G.H. Golub and C.F. Van Loan. Matrix Computations. Johns Hopkins University Press, 1993.

[44] E. Hagfors, T. Tereshchenko. Two-dimensional chirp mapping of delay and doppler spread targets. Radio Science, pages 1199-1203, September 1991.

[45] S. Haykin. Adaptive Filter Theory. Prentice Hall, 1991.

[46] S. Haykin. Adaptive Filter Theory. Prentice Hall, 1996.

[47] Ho and Chang. A new configuration for convergence speedup in adaptive time delay estimation. IEEE Trans. on Signal Processing, pages 2683-, November 1992.

[48] Howe and Hinton. Acoustic burst transmission of high rate data through shallow underwater channels. IEE Electronic Letters, February 1992.

[49] Izzo and Napolitano. Interference tolerant estimation of amplitude and time delay parameters of a composite signal. In Eusipco '90, pages 103-, 1990.

[50] N.S. Jayant and P. Noll. Digital coding of waveforms. Prentice Hall, 1984.

[51] F.B. Jensen, W.A. Kuperman, M.B. Porter, and H. Schmidt. Computational Ocean Acoustics. AIP Press, 1994. 
[52] M. Johnson, D. Brady, and M. Grund. Reducing the computational requirements for adaptive equalization in underwater acoustic communications. In Proc. Oceans'95, 1995.

[53] M. Johnson, L. Freitag, and M. Stojanovic. Improved doppler tracking and correction for underwater acoustic communication. In Proc. ICASSP'97, 1997.

[54] T. Kailath. Sampling models for linear time-variant filters. Technical report, MIT Research Lab, 1959.

[55] S.M. Kay. Fundamentals of statistical signal processing. Prentice Hall, 1993.

[56] A. Kaya and S. Yauchi. An acoustic communication system for subsea robot. In Proc. Oceans'89, pages 765-770, 1989.

[57] R.S. Kennedy. Fading Dispersive Communication Channels. Wiley, 1969.

[58] R.S. Kennedy and I.L. Lebow. Signal design for dispersive channels. IEEE Spectrum, March 1964, pages 231-237, March 1964.

[59] Knapp and Carter. Generalized cross correlation method for estimation of time delay. IEEE Trans. on Acoustics Speech and Signal Processing, pages 320-, August 1976.

[60] Krolik and Eizenman. Time delay estimation of signals with uncertain spectra. IEEE Trans. on Acoustics Speech and Signal Processing, pages 1801-, December 1988.

[61] H. Kwon and T. Birdsall. Digital waveform coding for ocean acoustic telemetry. IEEE Journal of Ocean Engineering, pages 56-66, January 1991.

[62] J. Lighthill. The fourth annual fairey lecture: The propagation of sound through moving fluids. Journal of sound and vibration, 24:471-492, 1972. 
[63] J. Lin, J. Proakis, F. Ling, and H. Lev-Ari. Optimal tracking of time-varying channels: A frequency domain approach for known and new algorithms. IEEE transactions on selected areas in communication, pages 141-153, January 1995.

[64] L. Ljung. System Identification Theory for the User. Prentice Hall, 1987.

[65] L. Ljung. General structure of adaptive algorithms: Adpatation and tracking. In N. Kalouptisidis and S. Theodoridis, editors, Adaptive system identification and signal processing algorithms. Prentice Hall, 1993.

[66] Lourtie and Moura. Multisource delay estimation: Nonstationary signals. IEEE Trans. on Signal Processing, pages 1033-, May 1991.

[67] J.N. Maksym. A robust formulation of an optimum cross-sprectral beamformer for line arrays. J. Acoust. Soc. Am., pages 971-975, April 1979.

[68] Messer and Bar-Ness. Closed loop time delay estimator. IEEE Trans. on Acoustics Speech and Signal Processing, pages 413-, April 1987.

[69] Morgera. Multiple terminal acoustic communication systems design. IEEE Journal of Ocean Engineering, July 1980.

[70] W.H. Munk, R.C. Spindel, A.B. Baggeroer, and T.G. Birdsall. The heard island feasibility test. J. Acoust. Soc. Am., pages 2330-2342, October 1994.

[71] F.D. Natali and W.J. Walbesser. Phase locked loop detection of binary psk signal utilizing decision feedback. IEEE Trans. Aerospace Electronic Syst., pages 83-90, January 1969.

[72] Nimier and Jourdain. Estimation of propagation and amplitude in active underwater acoustics. In Eusipco'90, 1990.

[73] Olson. Communication, control and data acquisition systems for the IHSTE lander. IEEE Journal of Ocean Engineering, January 1985. 
[74] O'Reilly. Error propagation in decision feedback receivers. IEE Comm. Radar and Sign.Proc., December 1985.

[75] Pallas and Jourdain. Active high resolution time delay estimation for large BT signals. IEEE Trans. on Signal Processing, pages 781-, April 1991.

[76] D.R. Palmer, M.G. Brown, et al. Classical ray chaos in nonseparable wave propagation problems. Geophysical research letters, pages 569-572, June 1988.

[77] J. Preisig. Reduced complexity adaptive array processing for multi-user communication. In Proc. Oceans'95, 1995.

[78] J. Proakis. Adaptive equalization techniques for acoustic telemetry channels. IEEE Journal of Ocean Engineering, January 1991.

[79] J. Proakis. Coded modulation for digital communications over rayleigh fading channels. IEEE Journal of Ocean Engineering, pages 66-74, January 1991.

[80] J.G. Proakis. Performance of coherent detection systems using decision directed channel measurements. IEEE Trans. Comm. Systems, pages 54-63, March 1964.

[81] J.G. Proakis. Advances in communication systems, chapter 2. Academic Press, 1975. Editor: Viterbi.

[82] J.G. Proakis. Digital Communications. McGraw-Hill, 1989.

[83] A.W. Rihaczek. Principles of high resolution radar. McGraw Hill, 1969.

[84] G. Sandsmark. Simulations of an adaptive equalizer applied to high speed ocean acoustic data transmission. IEEE Journal of Ocean Engineering, January 1991. 
[85] H. Schmidt, A.B. Baggeroer, et al. Environmentaly tolerant beamforming for high-resolution matched field processing: Deterministic mismatch. J. Acoust. Soc. Am., pages 1851-1862, October 1990.

[86] K.S. Shifrin. Physical optics of ocean water. American institute of physics, 1983.

[87] Simanin. Time delay estimation in multipath reception. Soviet Physics Acoustics, August 1991.

[88] Smith and Friedlander. Adaptive multipath delay estimation. IEEE Trans. on Acoustics Speech and Signal Processing, pages 812-, August 1985.

[89] R. Steele. Mobile radio communications. Wheatons Ltd., 1992.

[90] M. Stojanovic. Coherent Digital Communications for Rapidly Fading Channels with Applications to Underwater Acoustics. PhD thesis, Northeastern University, 1993.

[91] M. Stojanovic, J. Catipovic, and J. Proakis. Adaptive multichannel combining and equalization for underwater acoustic communications. J. Acoust. Soc. Am., pages 1621-1631, September 1993.

[92] M. Stojanovic, J. Catipovic, and J. Proakis. Phase-coherent digital communications for underwater acoustic channels. IEEE Journal of Ocean Engineering, pages 100-111, January 1994.

[93] M. Stojanovic, J. Proakis, and J. Catipovic. Analysis of the impact of channel estimation errors on the performance of a decision feedback equalizer in fading multipath channels. IEEE Trans. Comm. vol.43 no.2-4, pt.2, pages 877-886, 1995.

[94] G. Strang. Linear algebra. Wellesley-Cambridge Press, 1986. 
[95] M. Suzuki and T. Sasaki. Digital acoustic image transmission system for deep sea research submersible. In Proc. Oceans'92, pages 567-570, 1992.

[96] F.D. Tappret and X. Tang. Ray chaos and eigenrays. J. Acoust. Soc. Am., pages 185-195, January 1996.

[97] C.W. Therrien. Discrete random signals and statistical signal processing. Prentice Hall, 1992.

[98] D. Thompson et al. Performance of coherent PSK receivers using adaptive combining, beamforming and equalization in $50 \mathrm{~km}$ underwater acoustic channels. In Proc. Oceans'96, 1996.

[99] R.J. Urick. Principles of Underwater Sound. McGraw-Hill, 1975.

[100] H.L. van Trees. Detection, Estimation and Modulation Theory, pt.I. Wiley, 1968.

[101] H.L. van Trees. Detection, Estimation and Modulation Theory, pt.III. Wiley, 1968.

[102] Q. Wen and J. Ritcey. Spatial diversity equalization applied to underwater communications. IEEE Journal of Ocean Engineering, April 1994.

[103] B. Widrow and S.D. Stearns. Adaptive signal processing. Prentice Hall, 1985.

[104] A. Willsky. 6.432 stochastic processes detection and estimation. Supplementary course notes, MIT 1994.

[105] A. Willsky. 6.433 recursive estimation. Supplementary course notes, MIT 1995.

[106] P.M. Woodward. Probability and information theory with application to radar. Pergamon Press, 1953. 
[107] L.A. Zadeh. Frequency analysis of variable networks. Proceedings of IRE, pages 291-299, March 1950. 